\title{
Algebraic Structure and Integration in Generalized Differential Cohomology
}

\author{
Dissertation \\ zur Erlangung des mathematisch-naturwissenschaftlichen \\ Doktorgrades \\ "Doctor rerum naturalium" \\ der Georg-August-Universität Göttingen
}

im Promotionsprogramm PhD School of Mathematical Sciences der Georg-August University School of Science (GAUSS)

\author{
vorgelegt von \\ Markus Upmeier \\ aus Lawrence, KS (USA), \\ Göttingen, 2013
}


Betreuungsausschuss:

Prof. Dr. Thomas Schick, GRK 1493, Mathematisches Institut, Universität Göttingen

Prof. Dr. Ralf Meyer, GRK 1493, Mathematisches Institut, Universität Göttingen

Mitglieder der Prüfungskommision:

Referent: Prof. Dr. Thomas Schick, Mathematisches Institut, Universität Göttingen

Korreferent: Prof. Dr. Ralf Meyer, Mathematisches Institut, Universität Göttingen

Auswärtiger Gutachter: Prof. Dr. Stefan Schwede, Mathematisches Institut, Universität Bonn

Weitere Mitglieder der Prüfungskommision:

Prof. Dr. Karl-Henning Rehren, Institut für Theoretische Physik, Universität Göttingen

Prof. Dr. Dorothea Bahns, Mathematisches Institut, Universität Göttingen

Prof. Dr. Max Wardetzky, Institut für Numerische und Angewandte Mathematik, Universität Göttingen

Prof. Dr. Dominic Schuhmacher, Institut für Stochastik, Universität Göttingen

Tag der mündlichen Prüfung: 30.09.2013 


\section{Contents}

PREFACE

Notational Conventions . . . . . . . . . . . . . . . . . . . 8

\begin{tabular}{lll}
\hline & INTRODUCTION & 11
\end{tabular}

$1.1 \quad$ Ordinary Differential Cohomology . . . . . . . . . . . . . . . . 11

1.2 Generalized Differential Cohomology . . . . . . . . . . . . . . 18

1.3 Elementary Notions . . . . . . . . . . . . . . . . . . 20

2 Generalized Chern Character 22

2.1 Stable Homotopy Category . . . . . . . . . . . . . . . . . . 22

2.2 Construction . . . . . . . . . . . . . . . . . . . . 26

\begin{tabular}{lll}
\hline & DIFFERENTIAL COHOMOLOGY & 32 \\
\hline
\end{tabular}

3.1 Fundamental Cocycles . . . . . . . . . . . . . . . . . . . . 32

3.2 The Construction . . . . . . . . . . . . . . . . . . . . . . 34

3.3 Verification of the Axioms . . . . . . . . . . . . . . . . . . . . 39

3.4 Exact Sequence of Pairs . . . . . . . . . . . . . . . . . . . . . . . . . . . . . 43

3.5 Addition (Rationally Even Case) . . . . . . . . . . . . . . . . 46

4 FUNDAMENTAL GROUPOIDS OF COSPECTRA

4.1 Cospectra and the Category 2BrMonCat . . . . . . . . . . . . 48

4.2 Fundamental Groupoids of Loop Spaces . . . . . . . . . . . . . . 56

4.3 Construction of Cospectrum Groupoids . . . . . . . . . . . . . 62

$\begin{array}{llr}5 & \text { ADDITIVE STRUCTURES } & 66\end{array}$

5.1 The Cocycle Spectrum . . . . . . . . . . . . . . . . . 67

5.2 Chern Character Transformation . . . . . . . . . . . . . . . 73

5.3 The Additivity Coherence Conditions . . . . . . . . . . . . . . 79

5.4 Group Structure on Differential Cohomology . . . . . . . . . . 86

$5.5 \quad$ Bicategory Construction . . . . . . . . . . . . . . 87

$\begin{array}{llr}6 & \text { INTEGRATION MAPS } & \mathbf{9 0}\end{array}$

6.1 Slant Products and Integration over the Fiber . . . . . . . . . 91

6.2 Integration in Differential Cohomology . . . . . . . . . . . . . 94 
7 ACYCLIC MODELS THEOREM 104

7.1 Cohomological Version . . . . . . . . . . . . . . . . . . . 104

$7.2 \quad$ Applications . . . . . . . . . . . . . . . . . . . . . 109

8 Products (Rationally Even Case) 113

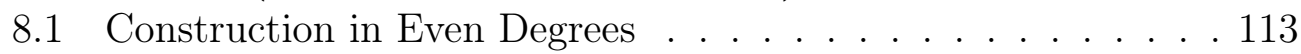

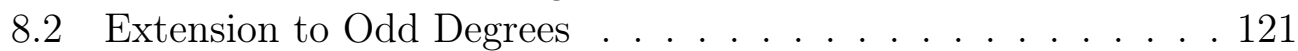

9 HighlY STRUCTURED DifFERENTIAL FUNCTION SPECTRA 125

9.1 Construction from Multiplicative Data . . . . . . . . . . 126

9.2 Canonical Multiplicative Data . . . . . . . . . . . . 132

10 UNIQUENESS (GENERAL CASE) 143

11 FIELD THEORY INTERPRETATION 145

11.1 Fields . . . . . . . . . . . . . . . . . . . 147

11.2 Push-Forward Maps. . . . . . . . . . . . . . . . . . . . . . 148

11.3 Integration on Closed Manifolds . . . . . . . . . . . . 150

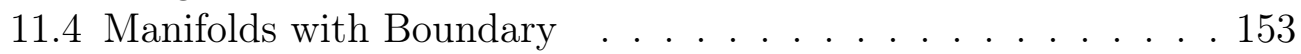

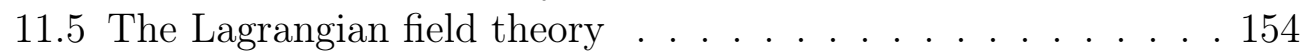

11.6 Outlook: Differential $K$-theory $\ldots \ldots \ldots . . \ldots \ldots$

\begin{tabular}{ll}
\hline A APPENDIX & 156
\end{tabular}

A.1 Braided Monoidal Categories . . . . . . . . . . . . . 156

A.2 Monoidal Structures under Equivalences . . . . . . . . . . 161

A.3 Simplicial Sets . . . . . . . . . . . . . . . . . . . . . . 166

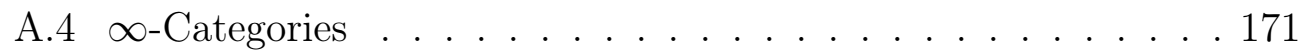

$\begin{array}{ll}\text { REFERENCES } & 178\end{array}$ 


\section{PREFACE}

The present thesis deals with the construction of algebraic structure, particularly products, on generalized differential cohomology from an abstract homotopy-theoretic point of view.

Beginning with a multiplicative cohomology theory $E$, we will show that the corresponding differential cohomology groups $\hat{E}$ are multiplicative in a suitable sense, which had been an open question for some time. The goal of this thesis is to provide two solutions to this problem:

First, we begin with a ring spectrum $E$ up to homotopy. We must then make some additional assumption that will guarantee various coherence conditions that lie at the heart of any construction of additional data on differential cohomology. A reasonable class is that of 'rationally even' cohomology theories and it contains most of the theories that arise in practice (the only notable exception being algebraic $K$-theory). We will show how our assumption implies half the coherence conditions we are after. We must also deal with the discrepancy between the cup product of cochains and the exterior product of differential forms. To this end we will prove, using standard ideas, a rather general cohomological Acyclic Models Theorem for 'corepresentable' functors (Theorems 7.9, 7.10) in Chapter 7, which may be applied to our situation. Combining this result with the coherence properties above, we are then able to work out products in even degrees (Theorem 8.7). It will then be our strategy to transport our construction from even to odd degrees (Theorem 8.19) by using so-called integration maps (Theorem 6.15), whose development is another important accomplishment of this thesis (Chapter 6).

In order to verify distributivity of the product and linearity of the integration map, we must have some information on the additive structure on the differential cohomology groups, which has unfortunately been constructed in [HS05. only in a completely abstract way: the diff. cohomology groups are realized there as homotopy groups of a spectrum which is, strictly speaking, obtained by applying fibrant replacement functors in a diagram model category (there is a choice of functorial section involved). In particular, no explicit structure maps are given. The lack of tractable 'deloopings' prohibits any understanding of addition. Moreover, the spaces of the differential function spectra don't seem to be Kan complexes, so the direct extraction of 
algebraic data is problematic at any rate.

We will spend considerable effort in Chapters 4 and 5 to construct an additive structure on the level of differential cocycles (without any assumptions on $E$ ), which is a major accomplishment of this thesis (Theorem 5.26). Working with simplicial sets, we may slightly remedy the situation with the structure maps above if we work with 'cospectra' instead of spectra. The main step is then the establishment of a refined generalized Chern character (Section 5.2)

$$
\operatorname{ch}: \operatorname{Hom}(K, E) \rightarrow Z\left(K \wedge \Delta^{\bullet}\right), \quad K \in \operatorname{Set}_{\Delta}
$$

which relates the mapping spectrum of $E$ with an algebraic construct that yields ordinary cohomology, which we call the cocycle spectrum. We will prove that both sides may be viewed as cospectra and that ch preserves the structure maps strictly. One may then extract parts of this information (using a fundamental groupoid construction - Chapter 4) to exhibit an addition on the level of differential cocycles.

Prior to this, we develop in Chapter 3 an explicit cocycle-based construction of generalized differential cohomology, whose definition is coarsely modeled on that of [HS05]. We will verify all the axioms for a differential refinement (Theorem 3.12) and develop some techniques for manipulating differential cocycles. Everything will be developed for pairs of spaces (which is crucial for the construction of the integration map later) and we will establish a certain long exact sequence for pairs (Theorem 3.15), which will prove very useful later.

This concludes the overview of our first approach.

The second solution is more abstract and views the differential function spectra as (homotopy) pullbacks in an $\infty$-category of spectra. It is our goal to give them the structure of commutative algebra objects (more precisely, commutative ring spectra valued presheaves on manifolds, that is, objects of the $\infty$-category Fun(Man $\left.\left.{ }^{\text {op }}, \mathrm{CAlg}(\mathrm{Sp})\right)\right)$. Roughly speaking, differential cohomology is a pullback of the diagram determined by the deRham homomorphism and the generalized Chern character. Bun12] deals with a refinement of the deRham homomorphism. The situation for the Chern character is 
much more involved (we need some hypothesis) and the necessary structure is assumed in Bun12 as additional data.

Note that the construction of $E_{\infty}$-structures is difficult at the best of times and is virtually impossible 'by hand.' We will need some large categorical machine for this and we will show how to squeeze the most out of the SchwedeShipley Theorem [SS03b] for this purpose. In Chapter 9 we will prove a (of course somewhat restricted) refinement (to the higher-categorical setting) of a Theorem of Dold Dol62 on the realization of maps between cohomology theories (Theorems 9.9 and 9.15). This will then be applied to construct, under a suitable hypothesis, a canonical (equivalence class) of a refinement of the generalized Chern character to an $E_{\infty}$-homomorphism (Subsection 9.2.5). Combined with the discussion in [Bun12], this refinement then determines a lift of the differential cohomology groups to spectra-valued presheaves. In particular, the corresponding homotopy groups are graded rings.

In Chapter 10 we investigate what can been said about uniqueness of differential refinements without restricting to the rationally even case. The main result (Theorem 10.3 may be viewed as a (weakened) generalization of a theorem by Bunke-Schick [BS10].

Integration over manifolds with boundary has not yet received a satisfactory treatment in differential cohomology. We will indicate in Chapter 11 (in the case of ordinary cohomology) that for this one should view differential cocycles as the 'fields' of a Lagrangian field theory and that integration of fields on manifolds with boundaries should be viewed as taking values in anomaly lines associated to the boundary. The results there explain also why it was not possible by Fre02 to construct the integral of differential cohomology classes independent of any choices. We interpret Chern-Simons theory and WZW-lines as the differential intersection pairing in this context, and show that the analogous construction for $K$-theory leads to $\eta$-invariants. The generalization of these arguments to other cohomology theories requires the use of $\infty$-categories and is a future project.

Chapter 1 serves as motivation and introduction to differential cohomology and includes some discussion on the history of the subject. In Chapter 2 we establish the classical generalized Chern character, which first appears in Dol62 (using different arguments). The appendix fixes terminology from (higher) category theory and the theory of simplicial sets used in this thesis. 
I would like to thank my advisor Thomas Schick for his constant support in my endeavors and his invaluable advice during my time as a doctoral student. Also, I would like to express my gratitude towards Ralf Meyer for supporting and advising me during this period, for pointing me towards the general treatment of uniqueness questions, and for encouraging me to use monoidal categories in the context of differential cohomology

\section{Notational Conventions}

The following standard (ordinary) categories will be denoted by bold face:

Man

$\operatorname{Man}^{2}$

$\mathrm{Ab}$

Set

Cat

$\operatorname{Mod}_{R}$

$\operatorname{Vect}_{\mathbb{R}}$

Ch

$\mathrm{Ch}_{\geq 0}$

$\mathrm{CoCh}$

$\mathrm{CoCh}^{\geq 0}$

cDGA

$\mathrm{s} \mathscr{C}$

$\mathscr{C}^{*}$

$\operatorname{Fun}(\mathscr{C}, \mathscr{D})$

$\operatorname{Set}_{\Delta}$

$\operatorname{Set}_{\Delta}^{+}$

Kan

CGHaus

VectBun
Manifolds and smooth maps

Pairs $(M, N)$ of a smooth manifolds $M$ with a closed submanifold $N \subset M$ and smooth maps of pairs

Abelian groups and group homomorphisms

Sets and set maps

Categories and functors

$R$-modules ( $R$ an ordinary ring) and $R$-module homomorphisms

Real vector spaces and $\mathbb{R}$-linear maps

Unbounded chain complexes and chain maps

Positive chain complexes and chain maps

Unbounded cochain complexes and cochain maps

Positive cochain complexes and cochain maps

Commutative differential graded algebras over $\mathbb{R}$

Category of simplicial objects in $\mathscr{C}$, that is, the functor

category s $\mathscr{C}=\operatorname{Fun}\left(\Delta^{\mathrm{op}}, \mathscr{C}\right)$

Pointed objects in $\mathscr{C}$, that is, the comma category $\mathscr{C}^{*}=(* \downarrow \mathscr{C})($ for $*$ final in $\mathscr{C})$

Functors $\mathscr{C} \rightarrow \mathscr{D}$ and natural transformations

Simplicial sets and simplicial maps

Marked simplicial sets

Kan complexes and simplicial maps

Compactly generated Hausdorff spaces and continuous maps

Real vector bundles and vector bundle homomorphisms 
hSp Stable homotopy category (see 2.1) and homotopy classes of 'maps' of spectra

$\operatorname{Alg}(\mathscr{C}), \mathrm{CAlg}(\mathscr{C})$ Category of (commutative) algebra objects in a (symmetric) monoidal category $\mathscr{C}$

$\operatorname{Ar}(\mathscr{C}) \quad$ Arrow category of $\mathscr{C}$ whose objects are morphisms in $\mathscr{C}$ and morphisms given by commutative squares

$\mathbf{h M o d}_{E} \quad$ Monoidal category of $E$-modules up to homotopy (for $E \in \mathrm{CAlg}(\mathbf{h S p})$ a commutative ring spectrum up to homotopy)

Coh Category of reduced additive cohomology theories (see 2.1) and transformations of cohomology theories

$\mathscr{C}^{\circ} \quad$ Full subcategory spanned by the cofibrant-fibrant objects in a model category $\mathscr{C}$.

coSpt Cospectra (Definition 4.1)

2BrMonCat Definition 4.4

MonCat Monoidal categories and strong monoidal functors (Subsection A.1.2

BrMonCat Braided monoidal categories and braided strong monoidal functors (Subsection A.1.3)

$\mathbb{R} / \mathbb{Z}$-Spaces $\quad$ Sets with a simply transitive $\mathbb{R} / \mathbb{Z}$-action and equivariant maps

HermLines Complex one-dimensional vector spaces with Hermitian metric and $\mathbb{C}$-linear maps preserving the metric

Note that for us 'monoidal functor' refers to what is sometimes called a strong (or weak - as opposed to (co)lax) monoidal functor. For an ordinary category $\mathscr{C}$, the set of morphisms will be written $\operatorname{Hom}_{\mathscr{C}}(x, y)$ or also $\mathscr{C}(x, y)$. If $\mathscr{C}$ is a $\mathcal{V}$-enriched category (for example, a simplicial category), the mapping object will be denoted $\operatorname{Map}_{\mathscr{C}}(x, y) \in \mathcal{V}$. If $K, L$ are simplicial sets (for example, $\infty$-categories), we will write $\operatorname{Hom}(K, L)$ for the internal hom, that is, the simplicial set

$$
\operatorname{Hom}(K, L)_{n}=\operatorname{Set}_{\Delta}\left(K \times \Delta^{n}, L\right) .
$$

Similarly, for pointed simplicial sets $K, L$ we write also $\operatorname{Hom}(K, L)$ for the (pointed) simplicial set whose $n$-simplices are simplicial maps

$$
K \wedge \Delta_{+}^{n} \rightarrow L
$$


Following [GJ09, we will write more generally $\operatorname{Hom}_{\mathscr{C}}(X, Y) \in \operatorname{Set}_{\Delta}$ for the mapping space in any simplicial model category $\mathscr{C}$.

If $L=\mathcal{C}$ is an $\infty$-category, $\operatorname{Hom}(K, L)$ is again an $\infty$-category, which is usually denoted by $\operatorname{Fun}(K, \mathcal{C})$. We will generally try to keep in line with the notation from [Lur09], [Lur11], and [GJ09].

$\mathcal{D}(\mathscr{A}) \quad$ Unbounded derived $\infty$-category of the abelian category $\mathscr{A}$

$\operatorname{Fun}(K, \mathcal{C}) \quad \infty$-category of diagrams in $\mathcal{C}$ of shape $K \in \operatorname{Set}_{\Delta}$

$\mathcal{S}$

Spaces. The simplicial nerve of the simplicial subcategory of Set $_{\Delta}$ spanned by the Kan complexes

$\mathcal{C}_{*} \quad$ Pointed objects of $\mathcal{C}$, the full subcategory of $\operatorname{Fun}\left(\Delta^{1}, \mathcal{C}\right)$ spanned by those edges $e: \Delta^{1} \rightarrow \mathcal{C}$ with $e(0)$ final in $\mathcal{C}$

Sp Spectrum objects in pointed spaces $\mathcal{S}_{*}$

$\mathrm{CAlg}(\mathrm{C}) \quad$ Category of commutative algebra objects in a symmetric monoidal $\infty$-category

$\operatorname{Mod}_{R} \quad$ Category of $R$-modules, $R \in \mathrm{CAlg}(\mathrm{C})$

$\mathrm{CAlg}_{R} \quad$ Category of $H R$-algebras, that is, $\mathrm{CAlg}{ }_{R}=\mathrm{CAlg}\left(\operatorname{Mod}_{H R}\right)$ for $R$ an ordinary ring

Equivalences will be denoted by ' $\simeq$ ' while the symbol ' $\cong$ ' is reserved for isomorphisms. The degree of an object $e$ (differential form, chain, etc.) will be written $|e|$.

$I$ denotes the unit interval $[0,1]$ whenever this makes sense. $S^{1}=\Delta^{1} / \partial \Delta^{1}$ as a simplicial set and $S^{1}=I / \partial I$ as a CW-complex. We will use standard notation for the following maps:

$\begin{array}{ll}\text { const } & \text { Constant map (usually with image a base-point) } \\ \text { incl } & \text { Inclusion of a subset } \\ \mathrm{pr}_{i} & \text { Projection from a product onto the } i \text {-th factor } \\ \text { id } & \text { Identity in a category } \\ \text { ev } & \text { Evaluation } \\ \text { can } & \text { Canonical projection }\end{array}$

If $(C, \delta)$ is a cochain complex we will write $C_{\delta}^{n}$ for the quotient group $C^{n} / \operatorname{im}\left(\delta^{n-1}: C^{n-1} \rightarrow C^{n}\right) . \Omega_{\mathrm{cl}}^{n}(M)$ denotes the group of closed $n$-forms on $M$ and $\Omega_{\mathrm{cl}, \mathbb{Z}}^{n}(M)$ the subgroup of those with integer periods. 


\section{INTRODUCTION}

\subsection{Ordinary Differential Cohomology}

One of the basic goals in topology is the distinction of spaces up to homeomorphism, diffeomorphism, or homotopy equivalence. The first sophisticated invariant of a space, the fundamental group, was defined by Poincaré more than 100 years ago. This concept has many extensions such as the homology groups $H_{*}(M)$ of a space. Roughly speaking, one investigates a space by embedding certain simple spaces into it and studying the resulting interrelations. Cohomology is the dual concept, elements of which typically 'classify' in some way topological objects defined over the space, such as characteristic classes of bundles or a 'bundle gerbe,' a higher-categorical generalization of a bundle.

On the other hand, classes in differential cohomology classify more geometric objects defined over the space, for example bundles with metrics and connections. These groups take the differential structure of the underlying manifold into account and are a much finer invariant of the situation, reflected by the presence of two homomorphisms

$$
\begin{aligned}
& I: \hat{H}^{*}(M) \rightarrow H^{*}(M), \\
& R: \hat{H}^{*}(M) \rightarrow \Omega_{\mathrm{cl}}^{*}(M) .
\end{aligned}
$$

Differential cohomology combines ideas from gauge theory (connections, curvature) and stable homotopy theory. For example, Chern-Weil theory combines gauge theory and ordinary cohomology. Early interest in differential cohomology [CS85] arose from the fact that the Chern-Weil homomorphism lifts to (ordinary) differential cohomology. In many cases this lift carries strictly more information than the Chern-Weil form and the characteristic class together, the example being that the holonomy of a flat bundle may be non-zero, even though the Euler form and class vanish (Example 1.7 .

The differential cohomology groups originate from the study of ChernSimons invariants of a bundle with connection and provide a natural home for these. Oftentimes these invariants are called secondary invariants, reflecting their dependence on an additional piece of data (the connection). Earlier still, Deligne considered a sheaf-theoretic definition of differential cohomology in the context of geometric quantization, which can serve as a starting point of a discussion on the physical significance of these groups. 


\subsubsection{Classical Gauge Theory}

The term 'gauge theory' refers to field theory in which a given physical situation is described by gauge potentials. Different gauge potentials that are related by a gauge transformation cannot be distinguished by any observer and are therefore equivalent descriptions of the same physical state. In practice, this often amounts to choosing antiderivatives of a physically observable quantity. Sometimes this allows to solve the field equations more conveniently. Let us consider electromagnetic field theory and the structure that naturally comes up when trying to choose antiderivatives:

Example 1.1 (Dirac's Magnetic Monopole). The electromagnetic field

$$
\vec{E}(\vec{x}, t), \vec{B}(\vec{x}, t)
$$

may be combined into a 2 -form $F \in \Omega^{2}(M)$ on Lorentzian space-time $M=$ $\left(\mathbb{R}^{4}, d x_{0}^{2}-d x_{1}^{2}-d x_{2}^{2}-d x_{3}^{2}\right)$. It satisfies a fully symmetric version! of Maxwell's equations

$$
d F=j_{B}, \quad d * F=j_{E}
$$

for the magnetic and electric currents $j_{B}, j_{E} \in \Omega^{3}(M)$, which contain information about where the charges are and where they are heading to.

As an example of a magnetic monopole, consider the following static electromagnetic field on $M=\mathbb{R} \times\left(\mathbb{R}^{3}\right)^{\times}$: the form

$$
F=g \frac{z d x d z+x d y d z+y d z d x}{r^{3}}
$$

is a solution to the Maxwell equations with $j_{E}=0$ and $j_{B}=0$. This field is considered to represent a magnetic monopole since by Gauss' theorem we have

$$
4 \pi g=\int_{\partial B_{r}(0)} F=\int_{B_{r}(0)} d F .
$$

In particular, $F$ is closed but not exact on the sphere $S^{2}=\partial B_{r}(0)$. Of course, we may locally choose gauge potentials $A_{+}, A_{-} \in \Omega^{1}\left(S_{ \pm}^{2}\right)$ on the upper and lower hemispheres, but these will not agree on the overlap. Instead, this data

\footnotetext{
${ }^{1}$ Many scientists believe that in nature there are no 'magnetic monopoles,' $j_{B}=0$, but some models of particle physics do predict the existence of particles with non-zero net magnetic charge.
} 
may be viewed as defining a principal circle bundle with connection on $S^{2}$ with curvature $F /(2 \pi i)$. The condition for this to work is $g \in \mathbb{Z}$ (being the first Chern number).

Apart from mere convenience, let us consider the following justification for studying gauge theory:

Example 1.2 (Quantum Mechanics). When passing to quantum mechanics one faces the problem that one has to choose a gauge potential $A \in \Omega^{1}(M)$ with $d A=F$ in order to perform the formal quantization. Different choices of potentials $A$ lead to formally different Schrödinger equations. Moreover, the Bohm-Aharonov experiment asserts that on the quantum level the gauge potentials actually have physical significance. In it, one observes that electrons traveling through a region in space in which the electromagnetic field vanishes may still experience a force from a field that is trapped inside a Faraday cage. The solution as proposed by Dirac is to replace the quantum Hilbert space $L^{2}\left(\mathbb{R}^{3}\right)$ by the space of sections of a line bundle $L$ on $\mathbb{R}^{3}$ Bry08. The point is that even though $L$ is trivializable, there is no preferred trivialization, or gauge. Any two trivializations will be related by a bundle automorphism. The Hamiltonian operator then involves a covariant derivative of a connection on $L$ given by the choice of $A$ (gauge potential) in the given local gauge.

\subsubsection{Motivation: Degree Two Differential Cohomology}

The structure appearing naturally in both these examples is that of a principal $S^{1}$-bundle with connection.

Definition 1.3. Let $\mathscr{H}^{2}(M)$ denote the groupoid of principal $S^{1}$-bundles $(P, \nabla)$ with connection on $M$. The morphisms from $\left(P_{1}, \nabla_{1}\right)$ to $\left(P_{2}, \nabla_{2}\right)$ are given by the set of all bundle maps $f: P_{1} \rightarrow P_{2}\left(\right.$ over id $\left.\operatorname{id}_{M}\right)$ with $f^{*} \nabla_{2}=\nabla_{1}$.

By the associated bundle construction, $\mathscr{H}^{2}(M)$ is equivalent to the category of Hermitian line bundles with connection on $M$. The tensor product of line bundles and connections gives rise to a symmetric monoidal structure on $\mathscr{H}^{2}(M)$. 
Definition 1.4. $\hat{H}^{2}(M)$ is the abelian group of equivalence classes of principal $S^{1}$-bundles on $M$ with connection: $\hat{H}^{2}(M)=\pi_{0} \mathscr{H}^{2}(M)$.

A gauge potential of $F \in \Omega_{\mathrm{cl}}^{2}(M ; i \mathbb{R})$ consists of a circle bundle with connection that has curvature $F$. A necessary and sufficient criterion for the existence of a gauge potential is that $F$ be integral (quantization condition). Moreover, any two gauge potentials will differ by a flat connection, which in turn are classified by homomorphisms $\pi_{1}(M) \rightarrow S^{1}$. We get an exact sequence

$$
0 \rightarrow H^{1}(M ; \mathbb{R} / \mathbb{Z}) \rightarrow \hat{H}^{2}(M) \stackrel{R}{\rightarrow} \Omega_{\mathrm{cl}, \mathbb{Z}}^{2}(M) \rightarrow 0 .
$$

Here, $\Omega_{\mathrm{cl}, \mathbb{Z}}^{2}(M)$ denotes closed differential 2-forms $\omega$ with integer periods, with $\int_{\sigma} \omega \in \mathbb{Z}$ for every cycle $\sigma$. On the other hand, a circle bundle is topologically classified by its first Chern class, and the space of connections is an affine space modeled on $\Omega^{1}(M)$. This leads to another exact sequence

$$
\Omega^{1}(M) / \operatorname{im}(d)=\Omega^{1}(M)_{d} \stackrel{a}{\rightarrow} \hat{H}^{2}(M) \stackrel{I}{\rightarrow} H^{2}(M ; \mathbb{Z}) \rightarrow 0 .
$$

Here, $a$ assigns the class of the trivial bundle $M \times S^{1}$ to $\theta \in \Omega^{1}(M)_{d}$ with the connection determined by $\theta$. To compute the kernel of $a$, we must understand gauge transformations $g: M \rightarrow S^{1}$ from the trivial bundle to itself that pull the connection $\theta$ back to zero, which solve

$$
g^{-1} d g=\theta
$$

Viewing the left-hand side as a logarithmic derivative, it is clear that we may always locally find $2 \pi i \mathbb{R}$-valued solutions $g$. The condition that the exponential of these local solutions agree on overlaps leads to an integrality condition on $\theta$, as expressed in the extended exact sequence

$$
H^{1}(M ; \mathbb{Z}) \rightarrow \Omega^{1}(M)_{d} \stackrel{a}{\rightarrow} \hat{H}^{2}(M) \stackrel{I}{\rightarrow} H^{2}(M) \rightarrow 0 .
$$

By the Chern-Weil Theorem, the maps $I$ and $R$ are equal upon post-composing to $H^{2}(M ; \mathbb{R})$. These properties of $\hat{H}^{2}$ motivate the following definition due to [SS08:

Definition 1.5. A differential extension of ordinary cohomology consists of functors on the category of manifolds

$$
\hat{H}^{*}: \operatorname{Man}^{\text {op }} \rightarrow \mathbf{A b}, \quad * \in \mathbb{Z}
$$


along with natural transformations

$$
\begin{aligned}
I: \hat{H}^{*} & \longrightarrow H^{*}(-; \mathbb{Z}), \\
R: \hat{H}^{*} & \longrightarrow \Omega_{\mathrm{cl}, \mathbb{Z}}^{*}, \\
a: \Omega^{*-1} / \operatorname{im}(d) & \longrightarrow \hat{H}^{*}, \\
b: H^{*-1}(-; \mathbb{R} / \mathbb{Z}) & \longrightarrow \hat{H}^{n} .
\end{aligned}
$$

These are required to fit into a commutative diagram (the unnamed maps come from the Bockstein sequence)

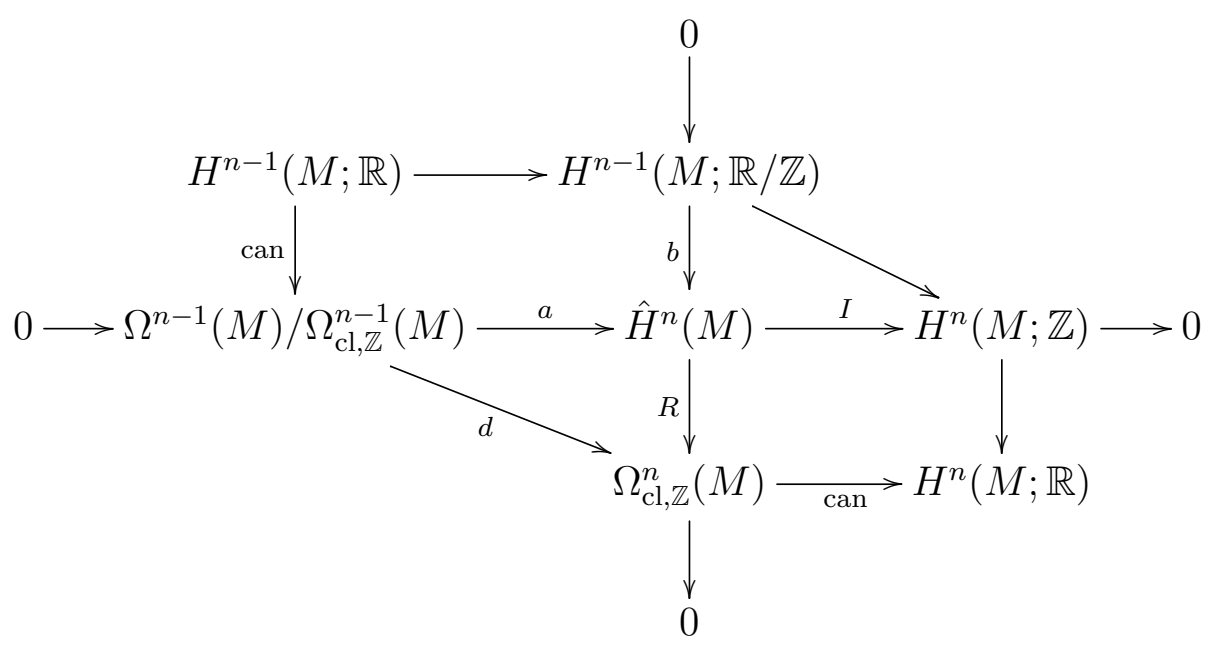

in which the middle vertical and middle horizontal sequence are exact.

\subsubsection{Cheeger-Simons Differential Characters}

The construction in the preceding section applies only to 2 -forms $F$. There exist higher-categorical objects (bundle gerbes) that are analogous to circle bundles and have 'curvatures' that are 3-forms. Higher degrees are even more complicated, the definitions being based on Čech-type descriptions of generalized 'bundles with connection.' While it is possible to carry this generalization out in detail, we shall proceed in a more elementary fashion. This is the approach of Cheeger-Simons [CS85] by what are called differential characters. 
Definition 1.6. Let $M$ be a smooth manifold. The $n$-th (ordinary) differential cohomology group of $M$ is defined as

$$
\hat{H}^{n}(M)=\left\{(u, \omega) \in \operatorname{Hom}_{\mathbf{A b}}\left(Z_{n-1}(M), \mathbb{R} / \mathbb{Z}\right) \times \Omega^{n}(M) \mid u \circ \partial=\omega \bmod \mathbb{Z}\right\} .
$$

In detail, we require that the homomorphism $u$ on the group $Z_{n-1}(M)$ of smooth $(n-1)$-cycles on $M$ be given on boundaries $\partial \sigma$ by integrating the differential $n$-form $\omega$ over $\sigma$ :

$$
\int_{\sigma} \omega=u(\partial \sigma) \quad \bmod \mathbb{Z}
$$

Example 1.7. Given a circle bundle with connection $(P, \nabla)$ on $M$, there is an element $u \in \hat{H}^{2}(M)$ which maps a closed curve $\gamma$ to its holonomy $\in S^{1}$. In case $\gamma$ is the boundary curve of a surface $\sigma$, then the holonomy may be computed by integrating the curvature form $\omega$ over $\sigma$. We observe that the holonomy $u$ may be non-zero, even when both the curvature $\omega$ and the characteristic class of $P$ vanish (for example $M=S^{1}$ ).

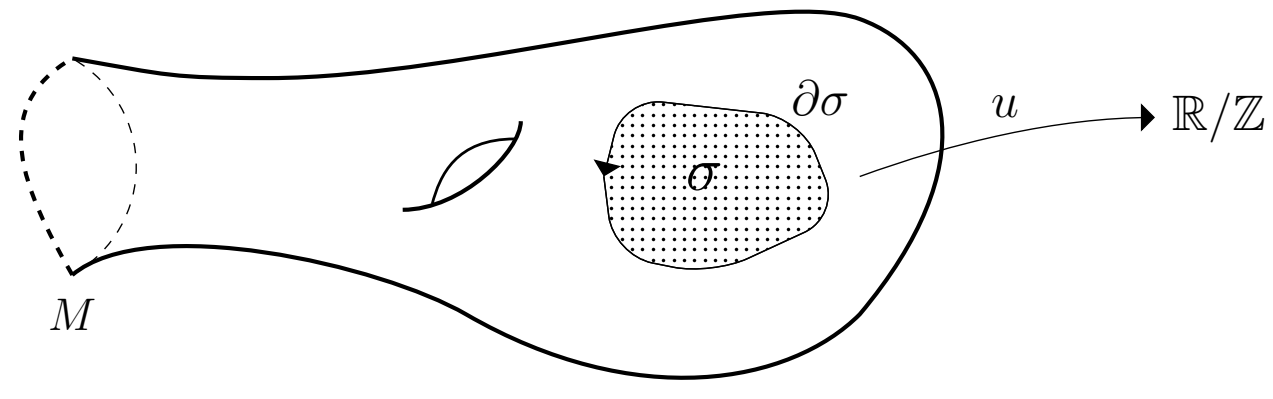

Lemma 1.8. For $n>0$ the form $\omega$ in (5) is unique.

Proof. Suppose $\omega \in \Omega^{n}(M)$ has the property that $\int_{\sigma} \omega \in \mathbb{Z}$ for each smooth $\sigma: \Delta^{n} \rightarrow M$. We wish to show $\omega=0$. Given $p \in M$, choose $\sigma: \Delta^{n} \rightarrow M$ with $p \in \sigma\left(\left(\Delta^{n}\right)^{\circ}\right)$. Pulling back along $\sigma$ and choosing a chart $\left(\Delta^{n}\right)^{\circ} \approx \mathbb{R}^{n}$ we are reduced to showing that a smooth function $f: \mathbb{R}^{n} \rightarrow \mathbb{R}$ whose integral over every simplex $\sigma: \Delta^{n} \rightarrow \mathbb{R}^{n}$ is an integer must vanish. By choosing a descending sequence of simplices around any point of $\mathbb{R}^{n}$, this follows easily from the continuity of $f$ and by approximating the integral by some value of 
$f$ times the volume of $\sigma$.

Applying the argument of the Lemma to $d \omega$, we conclude that a form $\omega$ as in (5) must be closed.

Definition 1.9. For $u \in \hat{H}^{n}(M)$ choose $\omega$ as in (5). Define

$$
R(u)=\omega \in \Omega_{\mathrm{cl}, \mathbb{Z}}^{n}(M) .
$$

Choose a real-valued lift of $u$ to a map $\hat{u}$. By choice of $\omega$, the cochain $W=\omega-\hat{u} \circ \partial: C_{n}(M) \rightarrow \mathbb{Z}$ takes values in $\mathbb{Z}$. It also vanishes on boundaries and we set

$$
I(u)=[W] \in H^{n}(M ; \mathbb{Z}) .
$$

Finally, for $\theta \in \Omega^{n-1}(M)$ and $w \in H^{n-1}(M ; \mathbb{R} / \mathbb{Z})$ we set

$$
\begin{aligned}
a(\theta): Z_{n-1}(M) \rightarrow \mathbb{R} / \mathbb{Z}, \quad \sigma \mapsto \int_{\sigma} \theta \bmod \mathbb{Z}, \\
b(w): Z_{n-1}(M) \rightarrow \mathbb{R} / \mathbb{Z}, \quad \sigma \mapsto w(\sigma) .
\end{aligned}
$$

Note that $b(w)$ vanishes on boundaries, so that it indeed belongs to $\hat{H}^{n}(M)$ and also $R b(w)=0$. The composition $I \circ b$ is essentially the definition of the Bockstein homomorphism.

By Stokes' Theorem, $a(\theta)$ is given on boundaries by $d \theta$, so that it too belongs to $\hat{H}^{n}(M)$ and we have $R a(\theta)=d \theta$. Since $\hat{u}=\sigma \mapsto \int_{\sigma} \theta$ is a real-valued lift of $a(\theta)$, we have $I a(\theta)=0$.

Theorem 1.10. The groups $\hat{H}^{n}(M)$ of Definition 1.6 and the transformations $R, I, a, b$ of Definition 1.9 are a differential extension of ordinary cohomology.

Proof. It remains to prove the commutativity of the two squares in (4) and exactness. Since $\hat{u} \circ \partial$ is a real coboundary, the maps $R$ and $I$ agree upon mapping to real cohomology. For the other square, let $x \in C_{n-1}(M) \rightarrow \mathbb{R}$ represent a class in $H^{n-1}(M ; \mathbb{R})$ and choose $\theta \in \Omega^{n-1}(M)$ cohomologous to $x$ (viewed as a real cochain). Then both $x$ and $\theta$ agree on cycles, so the other square commutes as well.

The exactness of the vertical sequence is trivial and the exactness of the horizontal sequence is a direct consequence of deRham's Theorem. 
Example 1.11 (Generalization of Example 1.7). Let $G$ be a Lie group with Lie algebra $\mathfrak{g}$. Given an invariant polynomial $f$ on $\mathfrak{g}$, the (absolute) ChernSimons form on a $G$-principal bundle with connection $(P \rightarrow M, \nabla)$ is a canonical choice of antiderivative of the pullback of the Chern-Weil form $f\left(\nabla^{2}\right) \in \Omega^{*}(M)$ to the total space $P$. As a consequence, one cannot compare them for different principal bundles $P$, even if they have the same base space. However, the mod $\mathbb{Z}$-reduction of the cochain corresponding to the Chern-Simons form may be viewed as an element $u \in \hat{H}^{*}(M)$, a group intrinsically assigned to the base space. This is why differential cohomology is regarded to be the 'natural home' for the Chern-Simons 'form.' The ChernWeil form $f\left(\nabla^{2}\right)$ can be recovered by taking the 'curvature' $R(u)$, and $I(u)$ is the characteristic class of $P$ belonging to $f \in H^{*}(B G ; \mathbb{R})$.

\subsection{Generalized Differential Cohomology}

\subsubsection{Introduction}

Generalized cohomology theories have their roots in index theory ( $K$-theory) and in Thom's work on cobordisms. In essence, generalized cohomology classes are invariants attached to topological situations constructed from the manifold. For example, the families index is associated to a family of elliptic differential operators over a manifold and represents a $K$-theory class.

Now again, generalized differential cohomology is a refinement of generalized cohomology theory that takes additional geometric features into account. Its first incarnation was in the form of differential $K$-theory and allows for a refined statement of the index theorem. The classical Atiyah-Singer Index Theorem for families asserts the equality of the analytic index of a family of Dirac operators with a cohomological quantity, involving the Chern character of the symbol and an $\hat{A}$-class. In the presence of 'additional geometry' one may pick canonical Chern-Weil representative differential forms. The Differential Index Theorem [BS09], [FL10] asserts that actually more (in the sense of Example 1.7) is true: one may lift both the analytical and topological index to differential cohomology and these refined indices coincide.

Roughly speaking, the objects of generalized differential cohomology represent gauge potentials of fields $F \in \Omega^{n}(M ; V)$ whose 'charges' have been lifted along the generalized Chern character (Chapter 2)

$$
\operatorname{ch}: E^{*}(M) \rightarrow H^{*}(M ; V), \quad V=E^{*}(\mathrm{pt}) \otimes \mathbb{R} .
$$


Here, $E^{*}$ denotes a generalized cohomology theory, such as $K$-theory or cobordism theory.

\subsubsection{Axiomatic Approach}

For ordinary integer cohomology the axioms in Definition 1.5 were presented by [SS08]. By analyzing only these axioms, they proved that the various constructions of differential cohomology were in fact isomorphic: the sheaftheoretic Deligne cohomology Gaj97, Cheeger-Simons differential characters [CS85], a stratifold model by Bunke-Kreck-Schick [BKS10], and the homotopy theoretic model of Hopkins-Singer [HS05].

Subsequently, BS10] considered the case of generalized cohomology. Let $E$ be a generalized cohomology theory in the sense of the Eilenberg-Steenrod axioms and let $V^{*}=E^{*}(\mathrm{pt}) \otimes_{\mathbb{Z}} \mathbb{R}$ denote the graded coefficient vector space.

Definition 1.12 ([BS10]). A differential extension of $E$ consists of functors

$$
\hat{E}^{*}: \operatorname{Man}^{\text {op }} \rightarrow \mathbf{A b}, \quad * \in \mathbb{Z}
$$

together with natural transformations

$$
\begin{aligned}
I: \hat{E}^{*} & \longrightarrow E^{*}, \\
R: \hat{E}^{*} & \longrightarrow \Omega_{\mathrm{cl}}^{*}(-, V), \\
a: \Omega^{*-1}(-, V) / \operatorname{im}(d) & \longrightarrow \hat{E}^{*} .
\end{aligned}
$$

These are required to fit into a commutative diagram

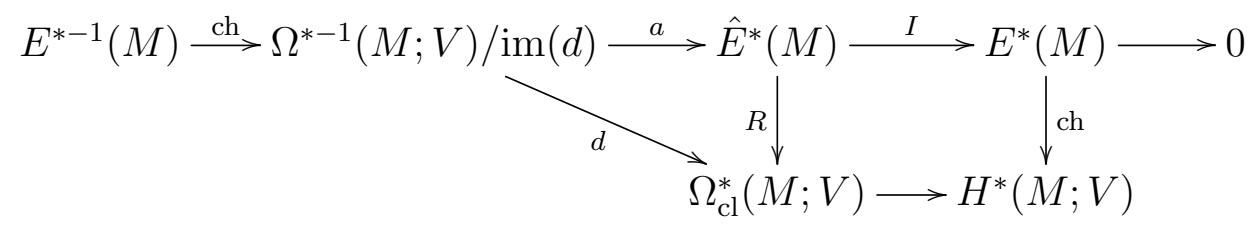

with an exact upper horizontal line. Such a differential extension is called multiplicative if $\hat{E}$ is a functor into graded commutative rings, if $I$ and $R$ are unital ring homomorphisms, and if

$$
a(\theta) \cup \hat{x}=a(\theta \wedge R(\hat{x})) \quad \forall \theta \in \Omega^{n-1}(M ; V), \hat{x} \in \hat{E}^{m}(M) .
$$


The generalized Chern character ch: $E^{*}(M) \rightarrow H^{*}(M ; V)$ will be carefully constructed in Section 2 .

Example 1.13 (Differential $K$-Theory). Following [FL10, the $0^{\text {th }}$ differential $K$-theory group $\hat{K}^{0}(M)$ may be identified with a quotient of the set of Hermitian vector bundles with connection on $M$ equipped with an element $\phi \in \Omega^{\text {odd }}(M) / \operatorname{im}(d)$. Then $R$ takes $(E, \nabla, \phi)$ to $\operatorname{ch}\left(\nabla^{2}\right)-d \phi$, essentially the Chern-Weil representative of the Chern character. The map $I$ simply retains the ordinary $K$-theory class.

An isomorphism of differential cohomology theories is defined to be a natural transformation which is compatible with the maps $a, R, I$. One of the main results in [BS10] is the following theorem. It justifies the axiomatic approach presented above, but note the assumptions on the cohomology theory $E$ :

Theorem 3.10 from [BS10]. Suppose $E$ is rationally even, that is, $E$ is a cohomology theory whose graded coefficient group $E^{*}(\mathrm{pt})$ is torsion in all odd degrees. If $E^{*}(\mathrm{pt})$ is countably generated in all degrees, then any two differential extensions $\check{E}$ with integration will be isomorphic via a unique isomorphism respecting integration.

We shall see in Chapter 10 how uniqueness may be formulated in the case of an arbitrary cohomology theory. For this we must introduce a weaker notion of 'equivalent' differential cohomology theories.

\subsection{Elementary Notions}

\subsubsection{Manifolds with Corners}

We will need to extend the functor $\Omega^{*}(M ; W)$ to the category Man of manifolds with corners, that is, second countable Hausdorff spaces with a smooth structure modeled on 'Euclidean corners'

$$
\square_{n}=\left\{\left(x_{1}, \ldots, x_{n}\right) \in \mathbb{R}^{n} \mid x_{1}, \ldots, x_{n} \geq 0\right\} .
$$

A map on an open subset $U$ of a Euclidean corner is said to be smooth if it allows a smooth extension to some open subset of $\mathbb{R}^{n}$ containing $U$. With 
this extended notion of smoothness, charts and atlases may be defined as usual. The tangent bundle is the unique up to isomorphism functor

$$
T: \text { Man } \rightarrow \text { VectBun with } T \square_{n} \cong \square_{n} \times \mathbb{R}^{n} .
$$

In particular, it makes sense to consider differential forms on manifolds with corners.

Example 1.14. The standard simplex $\Delta^{n}$ is an $n$-manifold with corners. An atlas is given by the collection $i=0, \ldots, n$ of homeomorphisms

$$
\begin{aligned}
\left\{\left(t_{0}, \ldots, t_{n}\right) \in \Delta^{n} \mid t_{i} \neq 0\right\} & \rightarrow\left\{\left(x_{1}, \ldots, x_{n}\right) \in \square_{n} \mid \sum x_{i}<1\right\} \\
\left(t_{0}, \ldots, t_{n}\right) & \mapsto\left(t_{0}, \ldots, t_{i-1}, t_{i+1}, \ldots, t_{n}\right) .
\end{aligned}
$$

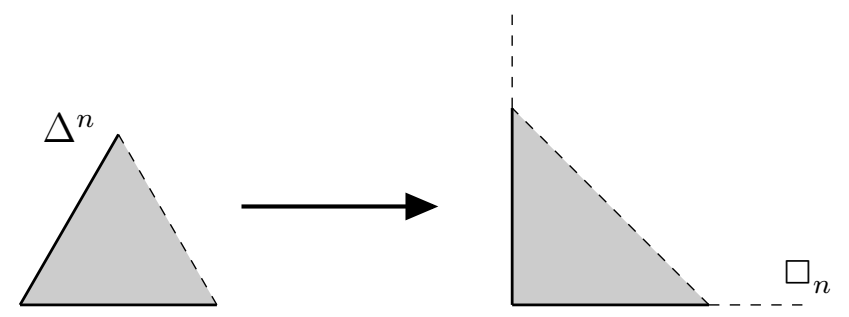

Note that there are several competing definitions for 'manifold with corners.' The one presented here is briefly discussed in the textbook [Lee03] and called 't-manifolds' in [HS05, C.1].

\subsubsection{Smooth Chains and Cochains}

For any $M \in$ Man one may form the smooth singular set $\operatorname{sing}_{\mathrm{s}}(M)$. whose $n$-simplices are smooth maps

$$
\sigma: \Delta^{n} \rightarrow M
$$

Passing to the associated Moore complex leads to the smooth chain groups

$$
C_{n}^{\mathrm{s}}(M)=\bigoplus\left\{\mathbb{Z} \mid \sigma: \Delta^{n} \rightarrow M \in \operatorname{sing}_{\mathrm{s}}(M)_{n}\right\}
$$

Relative versions $C_{n}^{\mathrm{s}}(M, N)$ for submanifolds $N \subset M$ are constructed in the usual way by taking quotients. For any abelian group $A$, dualizing yields the cochain complex of smooth cochains

$$
C_{\mathrm{s}}^{n}(M ; A)=\operatorname{Hom}_{\mathbf{A b}}\left(C_{n}^{\mathrm{s}}(M), A\right) .
$$

Note that any singular cochain may be restricted to a smooth cochain. 


\subsubsection{Relative de Rham Complex}

For a closed submanifold $i: N \subset M$ we let $\Omega^{*}(M, N)=\left\{\omega \in \Omega^{*}(M) \mid i^{*} \omega=0\right\}$. We include the case $N=\emptyset$ where we set $\Omega^{*}(\emptyset)=0$. There is a short exact sequence

$$
0 \rightarrow \Omega^{*}(M, N) \rightarrow \Omega^{*}(M) \rightarrow \Omega^{*}(N) \rightarrow 0
$$

of cochain complexes, which of course yields a corresponding long exact sequence in cohomology. Integrating smooth simplices allows us to view differential forms as smooth singular cochains

$$
\int: \Omega^{n}(M, N) \longrightarrow C_{\mathrm{s}}^{n}(M, N),\left(\int \omega\right) \sigma=\int_{\sigma} \omega .
$$

By the five-lemma and the long exact sequences we have the relative de Rham isomorphism

$$
H_{\mathrm{dR}}^{n}(M, N) \stackrel{\cong}{\longrightarrow} H_{\mathrm{s}}^{n}(M, N) \stackrel{\cong}{\longleftarrow} H^{n}(M, N)
$$

\section{Generalized Chern Character}

The goal of this section is to establish the classical Chern Character. This is a transformation of cohomology theories that derives from the fact that over the reals there is an essentially unique cohomology theory. This is due to Dold [Dol62]. It derives from deep results of Serre on the stable homotopy groups of spheres, namely that

$$
\pi_{i}(\mathbb{S})= \begin{cases}i<0: & 0 \\ i=0: & \mathbb{Z} \\ i>0: & \text { finite group }\end{cases}
$$

\subsection{Stable Homotopy Category}

To formulate and establish the results in this section it will suffice to work in the stable homotopy category $\mathbf{h S p}$, whose main features we briefly recall.

Definition 2.1. A prespectrum is a sequence of pointed Kan complexes (or spaces) $E_{n}$ along with pointed structure maps $E_{n} \rightarrow \Omega E_{n+1}$. In case these are all weak equivalences, we speak of a spectrum (for emphasis also called $\Omega$-spectrum). 
The objects of the stable homotopy category are all prespectra, but morphisms are more complicated to define. In short, they are eventually defined 'maps' (equivalence classes of cofinally defined 'functions') up to eventually defined homotopy Ada95, Section III.2]. This category is equivalent to the category whose objects are all CW-spectra with morphisms given by homotopy classes of 'functions.' The category hSp has a closed symmetric monoidal structure given by the so-called smash product and an internal

$$
\wedge: \mathrm{hSp} \times \mathrm{hSp} \rightarrow \mathrm{hSp}, \quad \mathrm{hSp}^{\text {op }} \times \mathrm{hSp} \rightarrow \mathrm{hSp} .
$$

It is also a triangulated category (which roughly asserts the existence of sensible Puppe sequences), but we will only need that it is additive. The abelian group of morphisms will be denoted $[E, F]$. The 0 -th space functor

$$
\Omega^{\infty}: \mathbf{h S p} \rightarrow \operatorname{Ho}\left(\mathbf{S e t}_{\Delta}^{*}\right)
$$

has a left-adjoint $\Sigma^{\infty}: \operatorname{Ho}\left(\right.$ Set $\left._{\Delta}^{*}\right) \rightarrow \mathbf{h S p}$ called suspension spectrum. In addition, there is an adjoint equivalence

$$
\Sigma: \mathbf{h S p} \rightleftarrows \mathrm{hSp}: \Omega .
$$

While $\Sigma$ is compatible with $\Sigma^{\infty}$ and the usual suspension, $\Omega$ is compatible with $\Omega^{\infty}$ and the usual loop space. Finally, there is a symmetric monoidal functor called 'Eilenberg-MacLane spectrum'

$$
H: \mathbf{A b} \rightarrow \mathbf{h S p}
$$

Our presentation here is inspired by [Mal11, Ada95, and [Swi75, where the reader interested in more details is referred to.

Monoids in hSp are called ring spectra (up to homotopy). For each commutative ring spectrum $E$ we form the category of $E$-modules (up to homotopy) $\mathbf{h M o d}_{E}$ which inherits a (closed) monoidal structure $\wedge_{E}$ with unit object $E$ (see [HSS00] or [Ada95, p.320]). It is defined by the coequalizer

$$
M \wedge E \wedge N \rightrightarrows M \wedge N \rightarrow M \wedge_{E} N
$$

The monoid objects in this category are called E-algebras (up to homotopy) and form a category $\mathrm{Alg}\left(\mathbf{h} \mathbf{M o d} \mathbf{d}_{E}\right)$ (or $\mathrm{CAlg}\left(\mathbf{h M o d} \mathbf{M}_{E}\right)$ in the commutative case). 
Remark 2.2. The stable homotopy category $\mathbf{h S p}$ is but the first level of a much richer structure: a stable symmetric monoidal $\infty$-category Sp whose refined structure we will need later to establish more precise results. The homotopy category of $\mathrm{Sp}$ is equivalent to the stable homotopy category, but be warned that it is not true that the homotopy category of the $\infty$-category of algebra objects $\mathrm{Alg}(\mathrm{Sp})$ stands in the same relationship to $\mathrm{Alg}(\mathbf{h S p})$ ! Indeed, as remarked in [Sch07, p.114], the question of whether one may realize a ring spectrum up to homotopy as a symmetric ring spectrum involves many obstructions! Similarly, the homotopy category of the $\infty$-category $\operatorname{Mod}_{E}$ is not equivalent to $\mathbf{h M o d} \mathbf{E}_{E}$.

A cohomology theory satisfying Milnor's wedge axiom is also said to be additive [tD08, p.406]. This means that for any family of pointed spaces $X_{\alpha}$, $\alpha \in A$, the restriction maps induce an isomorphism

$$
\tilde{E}^{*}\left(\bigvee_{\alpha \in A} X_{\alpha}\right) \rightarrow \prod_{\alpha \in A} \tilde{E}^{*}\left(X_{\alpha}\right)
$$

In this situation, we have the Brown Representation Theorem:

Theorem 2.3 (9.27 and 9.13 in [Swi75] and [Bro62]). Let Coh denote the category of reduced cohomology theories with values in Ab satisfying Milnor's wedge axiom. Formation of reduced cohomology induces an essentially surjective, full (but not faithful) functor of categories

$$
\mathbf{h S p} \rightarrow \mathbf{C o h}, \quad E \mapsto \tilde{E}^{*}
$$

Remark 2.4. If $\tilde{E}^{*}$ is a multiplicative cohomology theory on the homotopy category of spectra, there exists a ring spectrum $E \in \mathbf{h S p}$ that gives rise to the product structure (13.70 and 13.71 in [Swi75]). The corresponding assertion for theories defined only on spaces seems to be false [Ada72, p.154].

The trouble with faithfulness is that from the equality of two morphisms $\tilde{E}^{*} \rightarrow \tilde{F}^{*}$ one may only deduce the existence of level-wise homotopies, but our homotopies need to commute with the structure maps. The non-faithfulness in the theorem gives rise to the theory of phantom maps. For example, there exists a non-trivial map $\Sigma^{\infty} \mathbb{C} P^{\infty} \rightarrow \Sigma^{\infty} S^{3}$ which induces the zero transformation of cohomology theories. 


\subsubsection{Ordinary Cohomology with Graded Coefficients}

Definition 2.5. Ordinary cohomology with coefficients in a graded real vector space $V$ is defined as (see for example [tD08, p. 520])

$$
H^{*}(X, A ; V)=\prod_{i \in \mathbb{Z}} H^{n-i}\left(X, A ; V^{i}\right) .
$$

It is important to use the product (not the direct sum) in order to obtain an additive cohomology theory for the establishment of the Chern character [Lüc05, 5.12]. Of course for finite-dimensional $X$ there is no difference between the product and the direct sum. Similarly, the generalized EilenbergMacLane spectrum $H V \in \mathbf{h S p}$ is (where $V^{*}=V_{-*}$ )

$$
H V=\prod_{i \in \mathbb{Z}} H V_{i}[i] \stackrel{\simeq}{\longleftarrow} \bigvee_{i \in \mathbb{Z}} H V_{i}[i] .
$$

The right hand equivalence is induced by the canonical map Ada95, Proposition III.3.14]. Explicitly, the $n$-th space of the spectrum $H V$ is

$$
\prod_{i \in \mathbb{Z}}\left(H V_{i}\right)_{i+n} .
$$

The adjoint structure maps of $H V$ are defined as the product of the adjoint structure maps of the individual spectra (this is because $\Omega$, being a right adjoint, commutes with limits).

Definition 2.6. Suppose $V$ is a graded commutative algebra. Cohomology classes $x \in H^{n}(X, A ; V), y \in H^{m}(X, B ; V)$ are represented by left-bounded sequences

$$
x^{i} \in H^{i}\left(X, A ; V^{n-i}\right), y^{j} \in H^{j}\left(X, B ; V^{m-j}\right), \quad i, j \geq 0 .
$$

We define $z=x \cup y \in H^{n+m}(X, A \cup B ; V)$ as the sequence

$$
z^{k}=\sum_{i+j=k}(-1)^{(n-i) j} x^{i} \cup y^{j} \in H^{k}\left(X, A \cup B ; V^{n+m-k}\right) .
$$

Since $i, j \geq 0$, this sum is finite. The units are given by one-term sequences (all other entries are zero)

$$
1_{X} \in H^{0}\left(X ; V^{0}\right) .
$$


The sign makes certain that we retain graded commutativity:

$(-1)^{(n-i) j} x^{i} \cup y^{j}=(-1)^{(n-i) j+i j+(n-i)(m-j)} y^{j} \cup x^{i}=(-1)^{m n}(-1)^{(m-j) i} y^{j} \cup x^{i}$.

If we define the boundary operators $\delta$ by $(\delta x)^{k}=\delta x^{k-1}$ then these definitions allow us to view $H^{*}(-; V)$ as a multiplicative cohomology theory [Lüc05, Definition 5.12] on pairs of CW-complexes.

The following will be needed in the proof of Theorem 2.8: it is not a fact about cohomology theories, but uses that we are working over the real numbers:

Lemma 2.7. Suppose $V$ is a graded vector space. Let $X_{\lambda}, \lambda \in L$, denote the poset of finite subcomplexes of $X$. The restriction maps induce an isomorphism

$$
H^{*}(X ; V) \cong \lim _{\longleftarrow} H^{*}\left(X_{\lambda} ; V\right)
$$

Proof. Using the Universal Coefficient Theorem, since homology and filtered colimits $X=\operatorname{colim} X_{\lambda}$ commute, and since any two limits commute:

$$
\begin{aligned}
H^{*}(X ; V) & =\prod_{i \in \mathbb{Z}} H^{*-i}\left(X ; V^{i}\right) \cong \prod_{i \in \mathbb{Z}} \operatorname{Hom}\left(H_{*-i}(X), V^{i}\right) \\
& \cong \prod_{i \in \mathbb{Z}} \operatorname{Hom}\left(\operatorname{colim} H_{*-i}\left(X_{\lambda}\right), V^{i}\right) \cong \prod_{i \in \mathbb{Z}} \lim \operatorname{Hom}\left(H_{*-i}\left(X_{\lambda}\right), V^{i}\right) \\
& \cong \lim \prod_{i \in \mathbb{Z}} \operatorname{Hom}\left(H_{*-i}\left(X_{\lambda}\right) ; V^{i}\right) \cong \lim \prod_{i \in \mathbb{Z}} H^{*-i}\left(X_{\lambda}, V^{i}\right) \\
& =\lim H^{*}\left(X_{\lambda} ; V^{i}\right) .
\end{aligned}
$$

\subsection{Construction}

The proof of the following theorem will occupy the bulk of this subsection. The first part is mentioned without proof in [Lüc05, 5.12]. It was first formulated in [Dol62, p.172 and p.175]

Theorem 2.8. Let $\tilde{E}$ be a reduced additive cohomology theory that takes values in real vector spaces. Then there is a canonical natural isomorphism 
of cohomology theories

$$
\tilde{E}^{*} \cong \tilde{H}^{*}(-; W), \quad W^{*}=\tilde{E}^{*}\left(S^{0}\right) .
$$

If $E$ is multiplicative, so is this transformation.

Note that $\tilde{E}^{*}(-) \otimes_{\mathbb{Z}} \mathbb{R}$ does generally not satisfy Milnor's wedge axiom [Lüc05, p.93], so we may not apply Theorem 2.8 directly to this cohomology theory. Instead, we will work with $E \wedge H \mathbb{R}$ which satisfies this hypothesis.

Corollary 2.9. For every $E \in \mathbf{h S p}$ there exists a canonical natural transformation from the associated cohomology theory

$$
\operatorname{ch}: \tilde{E} \rightarrow \tilde{H}^{*}(-; V), \quad V^{*}=\pi_{-*}(E) \otimes \mathbb{R} .
$$

If $E \in \mathrm{CAlg}(\mathbf{h S p})$, then ch is multiplicative.

Proof. The cohomology theory associated to $E \wedge H \mathbb{R}$ satisfies the conditions of Theorem 2.8, so we obtain a canonical isomorphism of cohomology theories $\widetilde{E \wedge H \mathbb{R}} \cong \tilde{H}^{*}(-; W)$ for $W^{*}=\widetilde{E \wedge H} \mathbb{R}^{*}\left(S^{0}\right)$. For finite CW-complexes $X$ we have Ada95, Proposition III.6.7]

$$
\widetilde{E \wedge H} \mathbb{R}^{*}(X) \cong \tilde{E}^{*}(X) \otimes_{\mathbb{Z}} \mathbb{R}
$$

from which the formula for the coefficients follows. Now precompose with the transformation associated to $E \rightarrow E \wedge H \mathbb{R}$.

Suppose next that $E \in \mathrm{CAlg}(\mathbf{h S p})$. Then $E \wedge H \mathbb{R}$ is an $H \mathbb{R}$-algebra spectrum (up to homotopy) and $E \rightarrow E \wedge H \mathbb{R}$ is a morphism in $\mathrm{CAlg}(\mathbf{h S p})$, which induces a multiplicative transformation of cohomology theories. By Theorem 2.8 the isomorphism $\widetilde{E \wedge H \mathbb{R}} \cong \tilde{H}^{*}(-; W)$ is multiplicative.

For any $\tilde{E} \in \mathbf{C o h}$ we may of course use Theorem 2.3 to choose an object $F \in \mathbf{h S p}$ representing it, but $F$ and $\tilde{E} \cong \tilde{F}$ are not canonical.

Example 2.10. Let $E=K$ be topological $K$-theory, which is defined as the cohomology theory represented by the spectrum $B U \times \mathbb{Z}$. For path-connected compact $X$, the Chern character $K(X) \rightarrow H^{\text {even }}(X ; \mathbb{R})$ takes the class of a line bundle $L \rightarrow X$ to tD08, p.520]

$$
\operatorname{ch}(L)=\sum_{i=0}^{\infty} \frac{c_{1}(L)^{n}}{n !} .
$$


If $L_{n}$ denotes the tautological line bundle over $X=\mathbb{C} P^{n}$ for $n=0,1, \ldots, \infty$, we have

$$
\operatorname{ch}\left(L_{n}\right)=1+X+X^{2} / 2 !+\ldots+X^{n} / n ! \in H^{\text {even }}\left(\mathbb{C} P^{n} ; \mathbb{R}\right)=\mathbb{R}[X] /\left(X^{n+1}\right) .
$$

Naturality implies

$$
\operatorname{ch}\left(L_{\infty}\right)=e^{X} \in H^{\text {even }}\left(\mathbb{C} P^{\infty} ; \mathbb{R}\right)=\mathbb{R} \llbracket X \rrbracket .
$$

We see again that it is important to take the product in Definition 2.5 because $e^{X}$ is not an element of $\mathbb{R}[X]$.

The cohomological Chern Character is somewhat tedious to establish:

Proof of Theorem 2.8. Since the coefficients of $H W$ and $E$ agree and both cohomology theories are additive, by a theorem of Milnor Swi75, Theorem 7.67] or by the Atiyah-Hirzebruch spectral sequence it suffices to construct a natural transformation of cohomology theories. The construction has two steps: first, we shall establish a natural transformation of cohomology theories $\prod_{i+j=n} \tilde{H}^{i}\left(X ; W^{j}\right) \rightarrow \tilde{E}^{n}(X)$ on finite pointed CW-complexes $X$. In this case, the product is a biproduct and we may define the map as a sum of wedges of generalized Hurewicz homomorphisms. Next, using that a CWcomplex $X$ is the colimit of its finite subcomplexes, we will define a natural transformation for general $X$.

Suppose that $X$ is a finite pointed complex. For $\left[X \wedge S^{l} \stackrel{f}{\rightarrow} S^{j+l}\right] \in$ $\pi^{j}\left(\Sigma^{\infty} X\right)$ consider the canonical map

$$
W^{i}=\tilde{E}^{i}\left(S^{0}\right) \cong \tilde{E}^{i+j+l}\left(S^{j+l}\right) \stackrel{f^{*}}{\rightarrow} \tilde{E}^{i+j+l}\left(X \wedge S^{l}\right) \cong \tilde{E}^{i+j}(X) .
$$

More explicitly, choose $E \in \mathbf{h S p}$ representing $\tilde{E}$. Elements $\left[S^{k} \stackrel{\sigma}{\rightarrow} E_{i+k}\right] \in$ $W^{i}$ and $\left[X \wedge S^{l} \stackrel{f}{\rightarrow} S^{j+l}\right] \in \pi^{j}\left(\Sigma^{\infty} X\right)$ are then paired together to an element of $\tilde{E}^{i+j}(X)$ represented by $(-1)^{k j}$ times the class of

$$
X \wedge S^{k+l} \rightarrow S^{k} \wedge X \wedge S^{l} \stackrel{\sigma \wedge f}{\longrightarrow} E_{i+k} \wedge S^{j+l} \rightarrow E_{i+k+j+l} .
$$

The sign ${ }^{2}$ ensures that this element only depends on the stable class of $\sigma$ and $f$ : replacing $f$ by its suspension clearly results in the suspension of the

\footnotetext{
${ }^{2}$ Predicted in the sense of [Sch07] by the occurence of $i+k+j+l$ in the 'wrong' order: $k$ and $j$ need to be exchanged in order to match $S^{k+l}$. Note that all signs depend on whether one takes suspensions on the left or on the right. We take them on the right.
} 
paired class. On the other hand, replacing $\sigma$ by $\sigma \wedge \mathrm{id}_{S^{1}}$, we need to compare the stable class of $(-1)^{(k+1) j}$ times the upper line with $(-1)^{k j}$ times the lower line in the following commutative diagram:

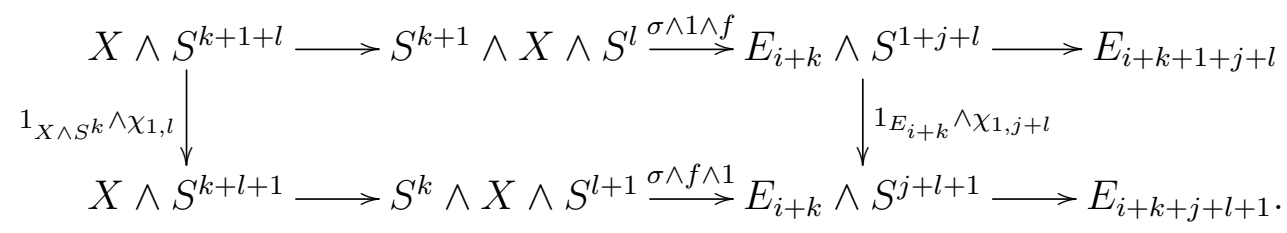

In this diagram, $\chi_{n, m}$ denotes the permutation of sphere coordinates according to the block permutation on $n+m$ letters of type $(n, m)$. Using Sch07, Lemma 2.3], we conclude that the upper and lower line represent stable classes that differ by $(-1)^{j}$, as desired.

These pairings (9) piece together to a natural transformation, compatible with suspension, on the biproduct

$$
h: \prod_{i+j=n} W^{i} \otimes \pi_{s}^{j}(X) \rightarrow \tilde{E}^{n}(X) .
$$

Comparing coefficients, we deduce that this map is an isomorphism ${ }^{3}$. It is important to remark here that the left hand side indeed is a cohomology theory on finite complexes (because stable cohomotopy is).

Suppose now that $E$ is multiplicative and choose maps $\mu_{i, j}: E_{i} \wedge E_{j} \rightarrow$ $E_{i+j}$ representing the product (well-defined up to homotopy). The left hand side of 10 is endowed with the product of graded algebras

$$
([\sigma] \otimes[f]) \cup([\tau] \otimes[g])=(-1)^{|f| \cdot|\tau|}([\sigma] \cup[\tau]) \otimes([f] \cup[g]) .
$$

Our transformation (10) is a homomorphism of graded rings: Unitality is clear. For multiplicativity, choose representatives

$$
\begin{array}{rlrl}
\sigma: S^{0} & \rightarrow E_{i} & {[\sigma]} & \in W^{i}, \\
\tau: S^{0} & \rightarrow E_{j} & {[\tau]} & \in W^{j}, \\
f: X \wedge S^{c} & \rightarrow S^{p+c} & {[f]} & \in \pi_{s}^{p}(X), \\
g: X \wedge S^{d} \rightarrow S^{q+d} & {[g]} & \in \pi_{s}^{q}(X) .
\end{array}
$$

\footnotetext{
${ }^{3}$ Milnor's Theorem is valid also for cohomology theories defined only on finite pointed CW-complexes [DK01, Theorem 8.31]; the proof follows by induction on the number of cells, the Mayer-Vietoris Sequence, and the Five Lemma.
} 
For simplicity, we may restrict to $\sigma, \tau$ being defined on $S^{0}$ (since $E$ is an $\Omega$-spectrum). Then we have $\mathrm{f}^{4}$

$$
\begin{aligned}
& {[\sigma] \cup[\tau]=\left[S^{0}=S^{0} \wedge S^{0} \stackrel{\sigma \wedge \tau}{\longrightarrow} E_{i} \wedge E_{j} \stackrel{\mu}{\rightarrow} E_{i+j}\right],} \\
& {[f] \cup[g]=(-1)^{c q}\left[X \wedge S^{c+d} \rightarrow X \wedge S^{c} \wedge X \wedge S^{d} \stackrel{f \wedge g}{\longrightarrow} S^{p+c+q+d}\right],} \\
& h([\sigma] \otimes[f])=\left[X \wedge S^{c}=S^{0} \wedge X \wedge S^{c} \stackrel{\sigma \wedge f}{\longrightarrow} E_{i} \wedge S^{p+c} \stackrel{\varepsilon}{\rightarrow} E_{i+p+c}\right], \\
& h([\tau] \otimes[g])=\left[X \wedge S^{d}=S^{0} \wedge X \wedge S^{d} \stackrel{\tau \wedge g}{\rightarrow} E_{j} \wedge S^{q+d} \stackrel{\varepsilon}{\rightarrow} E_{j+q+d}\right] .
\end{aligned}
$$

Thus, $h(([\sigma] \otimes[f]) \cup([\tau] \otimes[g]))=(-1)^{p j} h([\sigma] \cup[\tau] \otimes[f] \cup[g])$ is represented by $(-1)^{c q}$ times the stable class of the composition

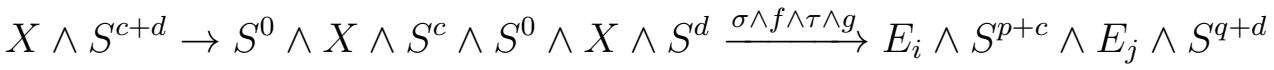

$$
\begin{aligned}
& \stackrel{\varepsilon \wedge \varepsilon}{\longrightarrow} E_{i+p+c} \wedge E_{j+q+d} \stackrel{\mu}{\longrightarrow} E_{i+p+c+j+q+d} .
\end{aligned}
$$

On the other hand, $h([\sigma] \otimes[f]) \cup h([\tau] \otimes[g])$ is represented by $(-1)^{c(j+q)}$ times the stable class of the composition

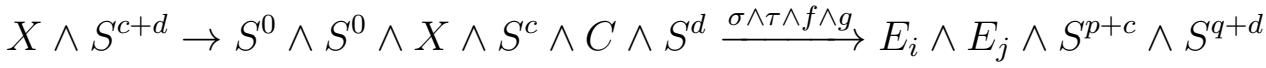

$$
\begin{aligned}
& \stackrel{\mu \wedge 1}{\longrightarrow} E_{i+j} \wedge S^{p+c+q+d} \stackrel{\varepsilon}{\longrightarrow} E_{i+j+p+c+q+d} .
\end{aligned}
$$

The diagram

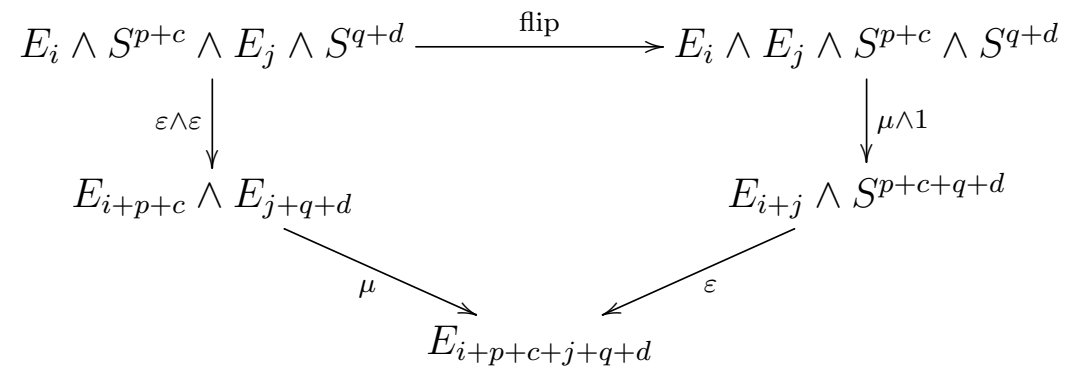

commutes up to sign $(-1)^{(p+c) j}$ (viewing both ways around as elements of the group $\left[E_{i} \wedge S^{p+c} \wedge E_{j} \wedge S^{q+d}, E_{i+p+c+j+q+d}\right]$; this follows from the compatibility of the multiplication on $\tilde{E}$ with suspensions). It follows that the stable classes of $(\mathrm{A})$ and $(\mathrm{B})$ differ by the factor $(-1)^{(p+c) j}$. Thus $(10)$ is

\footnotetext{
${ }^{4}$ The sign for $[f] \cup[g]$ arises since we take our suspensions on the right.
} 
multiplicative.

In the special case $\tilde{E}=\tilde{H} W^{j}$ we obtain the Hurewicz isomorphism $\pi_{s}^{*}(X) \otimes W^{j} \rightarrow \tilde{H}^{*}\left(X ; W^{j}\right)$. Taking the product of these and composing with 10 yields a natural isomorphism

$$
\tilde{H}^{n}(X ; W)=\prod_{i+j=n} \tilde{H}^{i}\left(X ; W^{j}\right) \cong \prod_{i+j=n} \pi_{s}^{i}(X) \otimes W^{j} \rightarrow \tilde{E}^{n}(X) .
$$

This concludes the first step.

The second step is formal. For a CW-complex $X$, let $\mathcal{L}_{X}$ denote the directed poset of finite $\mathrm{CW}$-subcomplexes $X_{\lambda} \subset X$. We consider the functors

$$
\tilde{E}^{n}, \tilde{H}^{n}(-; W): \mathcal{L}_{X}^{\text {op }} \rightarrow \operatorname{Vect}_{\mathbb{R}}
$$

The restrictions determine a canonical map

$$
\tilde{E}^{n}(X) \rightarrow \lim _{\lambda \in \overleftarrow{L}_{X}} \tilde{E}^{n}\left(X_{\lambda}\right)
$$

which is an isomorphism if $X$ is already finite (being then the initial object of $\mathcal{L}_{X}^{\mathrm{op}}$ ). Recall that by (8) the canonical map

$$
\tilde{H}^{n}(X ; W) \rightarrow \lim _{\lambda \in \overleftarrow{L}_{X}} \tilde{H}^{n}\left(X_{\lambda} ; W\right)
$$

is an isomorphism for any $X$. For each CW-complex $X$, the limit of the maps (11) therefore determine maps

$$
\tilde{E}^{n}(X) \rightarrow \lim _{\lambda \in \overleftarrow{\mathcal{L}}_{X}} \tilde{E}^{n}\left(X_{\lambda}\right) \rightarrow \lim _{\lambda \overleftarrow{\epsilon \mathcal{L}}_{X}} \tilde{H}^{n}\left(X_{\lambda} ; W\right) \cong \tilde{H}^{n}(X ; W)
$$

which generalize those for finite complexes. A map $f: X \rightarrow Y$ of $\mathrm{CW}$ complexes induces a functor $\mathcal{L}_{X} \rightarrow \mathcal{L}_{Y}$ and therefore determines maps

$$
\lim _{\mu \in \mathcal{L}_{Y}} \tilde{H}^{n}\left(Y_{\mu} ; W\right) \stackrel{f^{*}}{\rightarrow} \lim _{\lambda \leftarrow \mathcal{L}_{X}} \tilde{H}^{n}\left(X_{\lambda} ; W\right), \quad \lim _{\mu \in \overleftarrow{\mathcal{L}}_{Y}} \tilde{E}^{n}\left(Y_{\mu}\right) \stackrel{f^{*}}{\rightarrow} \lim _{\lambda \in \mathcal{L}_{X}} \tilde{E}^{n}\left(X_{\lambda}\right) .
$$

Explicitly, the component of $f^{*}$ for $\lambda \in \mathcal{L}_{X}$ is given by projecting to $Y_{\mu}=$ $f^{-1}\left(X_{\lambda}\right)$ and then using $f^{*}$ for finite complexes. With these definitions, 12 and (13) are natural transformations. From the naturality in the case of 
finite CW-complexes we conclude that (14) is natural. To show that (14) is compatible with suspensions it suffices to observe that $X_{\lambda} \wedge S^{1}$ for $\lambda \in L$ is a cofinal subcategory of $\mathcal{L}_{X \wedge S^{1}}$, so that we may take the corresponding limits in $(13)$ and 12 over these objects. Comparing coefficients, we conclude that (14) is an isomorphism of cohomology theories. It is multiplicative since the restriction maps are multiplicative and because this was true in the case of finite complexes.

\section{Differential Cohomology}

We will be concerned with the construction of differential cohomology theories $\hat{E}$ satisfying the axioms of Definition 1.12 (except the additive structure). The treatment in this section is completely elementary and requires no knowledge of abstract homotopy theory.

The additive structure is more difficult to see explicitly and will be developed in Section 5. This will be important for us since we will later construct products 'by hand' and this enables us to prove distributivity.

\subsection{Fundamental Cocycles}

\subsubsection{Setup}

We begin with a reduced additive cohomology theory $\tilde{E}^{*} \in$ Coh. Choose an $\Omega$-spectrum $\left(E_{n}, \varepsilon_{n}\right)$ representing $\tilde{E}$. We will require the adjoint structure maps

$$
\varepsilon_{n-1}^{\mathrm{adj}}: E_{n-1} \stackrel{\approx}{\longrightarrow} \Omega E_{n}
$$

to be pointed homeomorphisms (this can always be arranged).

Remark 3.1. Making a different choice of representing spectrum $\left(E_{n}, \varepsilon_{n}\right)$ leads to an isomorphic differential extension of $E$. They are not, however, canonically isomorphic. On the one hand, this makes the construction of additional structure so difficult. On the other hand, it is precisely this kind of behavior which 'enriches' differential cohomology as compared to classical cohomology theory. Otherwise one might suspect that the uniqueness result of [BS10] guarantees that nothing has been gained. This is not the case. 


\subsubsection{Implementation of the Chern Character}

Recall the generalized Chern character, which was a transformation

$$
\operatorname{ch}: \tilde{E}^{*} \longrightarrow \tilde{H}^{*}(-; V)
$$

of reduced cohomology theories. Since $\tilde{E}^{n}$ is represented by $E_{n}$, Yoneda's Lemma shows that the Chern character is implemented by pullback along the so-called fundamental cohomology classes $\left[\iota_{n}\right] \in \tilde{H}^{n}\left(E_{n} ; V\right)$ :

$$
\operatorname{ch}\left[X \stackrel{f}{\rightarrow} E_{n}\right]=E(f)^{*}\left[\iota_{n}\right]
$$

Since ch is a transformation of cohomology theories, the elements $\left[\iota_{n}\right]$ are related by the suspension and the structure maps and may therefore be viewed as an element of the group $\lim \tilde{H}^{n}\left(E_{n} ; V\right)$, the inverse limit being taken over

$$
\tilde{H}^{*+n}\left(E_{n} ; V\right) \stackrel{\varepsilon_{n-1}^{*}}{\longrightarrow} \tilde{H}^{*+n}\left(\Sigma E_{n-1} ; V\right) \stackrel{\text { susp }}{\longrightarrow} \tilde{H}^{*+n-1}\left(E_{n-1} ; V\right) .
$$

Lemma 3.2. $\lim \tilde{H}^{n}\left(E_{n} ; V\right)$ is isomorphic to the $0^{\text {th }}$ cohomology of the cochain complex $\lim C^{*+n}\left(E_{n}, \mathrm{pt} ; V\right)$, where the limit is taken over

$$
C^{*+n}\left(E_{n}, \mathrm{pt} ; V\right) \underset{\varepsilon_{n-1}^{*}}{\longrightarrow} C^{*+n}\left(\Sigma E_{n-1}, \mathrm{pt} ; V\right) \underset{\int_{S^{1}}}{\longrightarrow} C^{*+n-1}\left(E_{n-1}, \mathrm{pt} ; V\right) .
$$

Proof. Using universal coefficients, the fact that $\mathrm{Hom}_{\mathbb{R}}$ takes colimits in the first variable to limits, that any two limits commute, and that directed co- 
limits and homology commute, we calculate:

$$
\begin{aligned}
H^{n}\left(\lim C^{*+k}\left(E_{k}, \mathrm{pt} ; V\right)\right)=H^{n}\left(\lim \prod_{j \in \mathbb{Z}} C^{*-j+k}\left(E_{k}, \mathrm{pt} ; V^{j}\right)\right) \\
=H^{n}\left(\prod_{j \in \mathbb{Z}} \lim C^{*-j+k}\left(E_{k}, \mathrm{pt} ; V^{j}\right)\right)=\prod_{j \in \mathbb{Z}} H^{n}\left(\lim C^{*-j+k}\left(E_{k}, \mathrm{pt} ; V^{j}\right)\right) \\
=\prod_{j \in \mathbb{Z}} \operatorname{Hom}_{\mathbb{R}}\left(H_{n}\left(\operatorname{colim} C_{*-j+k}\left(E_{k}, \mathrm{pt}\right)\right), V^{j}\right) \\
=\prod_{j \in \mathbb{Z}} \operatorname{Hom}_{\mathbb{R}}\left(\operatorname{colim} H_{n}\left(C_{*-j+k}\left(E_{k}, \mathrm{pt}\right)\right), V^{j}\right) \\
=\prod_{j \in \mathbb{Z}} \lim \operatorname{Hom}_{\mathbb{R}}\left(H_{n}\left(C_{*-j+k}\left(E_{k}, \mathrm{pt}\right)\right), V^{j}\right) \\
=\lim \prod_{j \in \mathbb{Z}} H^{n}\left(C^{*-j+k}\left(E_{k}, \mathrm{pt} ; V^{j}\right)\right)=\lim H^{n}\left(\prod_{j \in \mathbb{Z}} C^{*-j+k}\left(E_{k}, \mathrm{pt} ; V^{j}\right)\right) \\
=\lim H^{n}\left(C^{*+k}\left(E_{k}, \mathrm{pt} ; V\right)\right)=\lim \tilde{H}^{n+k}\left(E_{k} ; V\right)
\end{aligned}
$$

If follows from Lemma 3.2 that we may choose fundamental cocycles $\iota_{n} \in$ $Z^{n}\left(E_{n}, \mathrm{pt} ; V\right)$ with the property that

$$
\iota_{n-1}=\int_{S^{1}} \varepsilon_{n-1}^{*} \iota_{n} .
$$

\subsection{The Construction}

Fix the data $\left(E_{n}, \varepsilon_{n}\right)$ from Subsection 3.1.1 and let $\iota_{n} \in Z_{\text {sing }}^{n}\left(E_{n}, \mathrm{pt} ; V\right)$ be a fixed choice of fundamental cocycles.

Definition 3.3. Let $N \subset M$ be a closed submanifold. The $n$-th differential E-cohomology $\hat{E}^{n}(M, N)$ is the set of equivalence classes of triples

$$
c:(M, N) \rightarrow\left(E_{n}, \mathrm{pt}\right), \quad \omega \in \Omega_{\mathrm{cl}}^{n}(M, N ; V), \quad h \in C_{s}^{n-1}(M, N ; V)
$$

satisfying

$$
\delta h=\omega-c^{*} \iota_{n} .
$$

An equivalence $\left(c_{0}, \omega_{0}, h_{0}\right) \sim\left(c_{1}, \omega_{1}, h_{1}\right)$ with $\omega_{0}=\omega_{1}$ is a pair

$$
C:[0,1] \times(M, N) \rightarrow\left(E_{n}, \mathrm{pt}\right), \quad H \in C_{s}^{n-1}([0,1] \times(M, N) ; V)
$$


with the properties

$$
\begin{gathered}
\delta H=\operatorname{pr}_{2}^{*} \omega-C^{*} \iota_{n} \\
i_{0}^{*} C=c_{0}, i_{1}^{*} C=c_{1}, \quad i_{0}^{*} H=h_{0}, i_{1}^{*} H=h_{1} .
\end{gathered}
$$

An equivalence class $[c, \omega, h]$ is called a differential cocycle.

Here, $i_{0}, i_{1}:(M, N) \rightarrow[0,1] \times(M, N)$ denote the inclusions of the endpoints and $\mathrm{pr}_{2}:[0,1] \times(M, N) \rightarrow(M, N)$ is the projection.

By pulling maps, forms, and cochains back along smooth maps we see that the differential cohomology sets may be organized into a functor

$$
\hat{E}^{n}:\left(\mathbf{M a n}^{2}\right)^{\mathrm{op}} \rightarrow \mathbf{S e t}_{*}
$$

into the category of pointed sets. The base-point is [const, 0,0$]$.

Remark 3.4. Two different choices of fundamental cocycles lead to isomorphic, but not canonically isomorphic differential cohomology theories. This makes the definition of structure on these groups very difficult, and every construction ultimately boils down to a compatibility statement for the fundamental cocycles. One might hope to obtain a family of fundamental cocycles compatible with the cup product structure by applying the approach of Lemma 3.2 to diagrams on index categories more sophisticated than $\mathbb{N}$. Unfortunately, the proof of this lemma relies on the fact that homology commutes with filtered colimits.

In general, one can obtain a spectral sequence which, when it collapses, guarantees that a compatible choice is possible:

\subsubsection{Aside: Spectral Sequence for the Simplicial Resolution of a Diagram}

Suppose $F: I \rightarrow \mathbf{C h}$ is a diagram of chain complexes of vector spaces, indexed on some category $I$. The standard simplicial resolution of $F$ is the simplicial chain complex $S \in s \mathbf{C h}$ with $n$-simplices

$$
S_{n}=\bigoplus_{i:[n] \rightarrow I} F\left(i_{0}\right)
$$


For $0<k \leq n$, the face maps $d_{k}$ on $S_{n}$ are given by 'covering up $i_{k}$.' Formally, $d_{k} \circ \operatorname{in}_{i}=\operatorname{in}_{i \circ d^{k}}$, where $\operatorname{in}_{i}: F\left(i_{0}\right) \rightarrow S_{n}$ denotes the canonical inclusion. The map $d_{0}$ is defined by

$$
d_{0} \circ \operatorname{in}_{i}=\operatorname{in}_{i \circ d^{0}} \circ F(i(0 \rightarrow 1)) .
$$

The simplicial object $S_{\bullet}$ may be visualized as follows:

$$
\bigoplus_{i_{0} \in \mathrm{Ob}(I)} F\left(i_{0}\right)_{*} \Longleftrightarrow \bigoplus_{i_{0} \rightarrow i_{1}} F\left(i_{0}\right)_{*} \Longleftrightarrow \bigoplus_{i_{0} \rightarrow i_{1} \rightarrow i_{2}} F\left(i_{0}\right)_{*} \quad \cdots
$$

From $S_{\bullet} \in s_{\mathbf{C h}}$ we may extract a double complex $T_{n *}=\left(S_{n}\right)_{*}$ with horizontal differential $\delta=\sum(-1)^{k} d_{k}$ and vertical differential $\partial=(-1)^{n} d^{S}$ : $\left(S_{n}\right)_{*} \rightarrow\left(S_{n}\right)_{*-1}$, where $d^{S}$ denotes the differential on $S_{n}$.

Of course the double complex induces a (homological) spectral sequence $\mathrm{McC01}$

$$
E_{p, q}^{2}=H_{p, q}(H(T, \partial), \delta) \Rightarrow H_{p+q}(\operatorname{Tot}(T)) .
$$

By first applying the vertical differential $\partial$ to $T$ we get

$$
\bigoplus_{i_{0} \in \mathrm{Ob}(I)} H_{*}\left(F\left(i_{0}\right)\right) \stackrel{\left(d^{0}\right)^{*}-\left(d^{1}\right)^{*}}{\leftarrow} \bigoplus_{i_{0} \rightarrow i_{1}} H_{*}\left(F\left(i_{0}\right)\right) \longleftarrow \delta \quad \bigoplus_{i_{0} \rightarrow i_{1} \rightarrow i_{2}} H_{*}\left(F\left(i_{0}\right)\right) \quad \cdots
$$

The first two columns of $T$ are just the coequalizer that is used to express an arbitrary colimit using coproducts and a coequalizer, hence $E_{0, q}^{2}=$ $\operatorname{colim}_{i \in I} H_{q}(F(i))$.

Since our spectral sequence is in the right half-plane, we have edge homomorphisms $E_{0, n}^{2} \rightarrow E_{0, n}^{\infty} \subset H_{n}(\operatorname{Tot}(T))$. Using the Universal Coefficient Theorem, these induce maps for every vector space $V$ (in $\operatorname{Vect}_{\mathbb{R}}$, monos dualize to epis and epis dualize to monos):

$$
\begin{aligned}
H^{n}(\operatorname{Tot}(T) ; V) & =\operatorname{Hom}\left(H_{n}(\operatorname{Tot}(T)), V\right) \rightarrow \operatorname{Hom}\left(E_{0, n}^{\infty}, V\right) \\
& \hookrightarrow \operatorname{Hom}\left(\operatorname{colim}_{i \in I} H_{n}(F(i)), V\right)=\lim _{i \in I} H^{n}(F(i) ; V)
\end{aligned}
$$

By taking direct products, (17) generalizes immediately to graded vector spaces $V$.

Proposition 3.5. If our spectral sequence collapses, (17) is surjective. 
A representative cocycle on the left may be interpreted as a coherent family of cocycles, refining the cohomology classes on the right. Indeed, an $n$-cocycle of $\operatorname{Hom}(\operatorname{Tot}(T) ; V)$ consists for each $0 \leq p \leq n$ and $\varphi:[p] \rightarrow I$ of a homomorphism

$$
f_{\varphi}: F\left(\varphi_{0}\right)_{n-p} \rightarrow V \text {, that is, cochains } f_{\varphi} \in C^{n-p}\left(F\left(\varphi_{0}\right), V\right) .
$$

These vanish on coboundaries of $\operatorname{Tot}(T)$, which implies 'coherence' relations among them. The above map (17) sends such a family to the element of the limit represented by the subfamily of cohomology classes of $f_{i:[0] \rightarrow I}: F(i)_{n} \rightarrow$ $V, i \in I$.

Unfortunately, there is no indication why the corresponding spectral sequence should collapse, except if we make homological assumptions on $E$.

\subsubsection{The Equivalence Relation}

To keep control over $\hat{E}^{n}(M, N)$ it is important to observe that ' $\sim$ ' is indeed an equivalence relation, so we don't have to pass to a 'generated' equivalence relation. It is obvious that the relation defined above is reflexive and symmetric (pull back along $\varphi \times \operatorname{id}_{(M, N)}$ for $\varphi:[0,1] \rightarrow[0,1], \varphi(t)=1-t$ ). To prove that our relation is transitive, we need a lemma on gluing cochains:

Lemma 3.6. Let $A \cup B=X$ be an open covering and $\tilde{A} \subset A, \tilde{B} \subset B$ be subsets with $\tilde{A} \cap B=\tilde{B} \cap A$. Then, restriction $C^{n}(A \cup B, \tilde{A} \cup \tilde{B}) \longrightarrow$ $C^{n}(A, \tilde{A}) \times_{C^{n}(A \cap B)} C^{n}(B, \tilde{B})$ is surjective. Similarly for smooth cochains.

Proof. Suppose $u \in C^{n}(A, \tilde{A}), v \in C^{n}(B, \tilde{B})$ have equal restriction to $A \cap B$. Consider the projection

$$
\pi: \Delta^{n+1} \rightarrow \Delta^{n},\left(t_{0}, \ldots, t_{n+1}\right) \mapsto\left(t_{0}+\frac{t_{n+1}}{n+1}, \ldots, t_{n}+\frac{t_{n+1}}{n+1}\right) .
$$



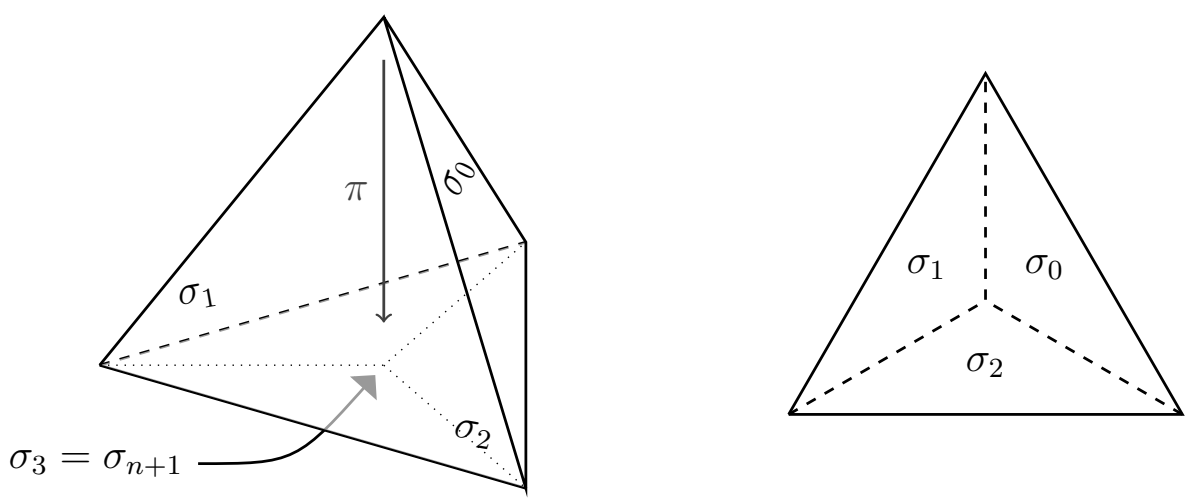

The coface map $d^{n+1}: \Delta^{n} \rightarrow \Delta^{n+1},\left(t_{0}, \ldots, t_{n}\right) \mapsto\left(t_{0}, \ldots, t_{n}, 0\right)$ then satisfies $\pi \circ d^{n+1}=\mathrm{id}_{\Delta^{n}}$. Let $\sigma_{n+1}: \Delta^{n} \rightarrow X$ be a simplex. For $\sigma:=\sigma_{n+1} \circ \pi$ let $\sigma_{i}=\sigma \circ d^{i}=\sigma_{n+1} \circ \pi \circ d^{i}$ so that we have

$$
\partial \sigma=\sigma_{0}-\sigma_{1}+\sigma_{2} \cdots+(-1)^{n+1} \sigma_{n+1} .
$$

We define a subdivision operator (not a chain map)

$$
\mathrm{sd}: C_{n}(X) \rightarrow C_{n}(X)
$$

on a basis by $\operatorname{sd}\left(\sigma_{n+1}\right)=\sigma_{n}-\sigma_{n-1} \cdots+(-1)^{n} \sigma_{0}=(-1)^{n} \partial \sigma+\sigma_{n+1}$. Our subdivision lets the diameters tend to zero and $\Delta^{n}$ is a compact metric space. Therefore after a finite minimal number $m=m(\sigma)$ of applications of sd any simplex $\sigma \in C_{n}(X)$ will be a chain $\sum_{k} n_{k} \cdot \tau_{k}$ consisting only of simplices $\tau_{k}$ whose image lies entirely in $A$ or entirely in $B$. We define $w(\sigma)$ as $\sum_{k} n_{k}\left\{\begin{array}{l}u\left(\tau_{k}\right) \text { if } \tau_{k}\left(\Delta^{n}\right) \subset A, \\ v\left(\tau_{k}\right) \text { if } \tau_{k}\left(\Delta^{n}\right) \subset B .\end{array} \quad\right.$ By the minimality assumption, $w$ restricts to $u$ and $v$ respectively; thus $w$ is preimage of $(u, v)$. This proves that the restriction map is surjective. We remark that our subdivision operator takes the subcomplex of smooth chains to itself.

Remark 3.7. Cocycles are invariant under the subdivision operator sd. Indeed, by definition $\operatorname{sd}\left(\sigma_{n+1}\right)=(-1)^{n} \partial \sigma+\sigma_{n+1}$ from which we conclude $u\left(\operatorname{sd}\left(\sigma_{n+1}\right)\right)=u\left((-1)^{n} \partial \sigma+\sigma_{n+1}\right)=u\left(\sigma_{n+1}\right)$. Closed differential forms are also invariant under sd.

Proposition 3.8. The above relation is an equivalence relation. 
Proof. We first remark that if $\left(c_{0}, \omega_{0}, h_{0}\right) \sim\left(c_{1}, \omega_{1}, h_{1}\right)$ then there exist $H, C$ as above such that $\left.C\right|_{[0,1 / 4]}=c_{0} \circ \mathrm{pr},\left.C\right|_{[3 / 4,1]}=c_{1} \circ \mathrm{pr},\left.H\right|_{[0,1 / 4]}=\operatorname{pr}^{*} h_{0}$, $\left.H\right|_{[3 / 4,1]}=\operatorname{pr}^{*} h_{1}$, so $H$ and $C$ are 'constant' near the endpoints. Indeed, given $C, H$ we may pull back along $\phi \times \operatorname{id}_{(M, N)}$ where $\phi$ is a smooth strictly increasing function $[0,1] \rightarrow[0,1]$ with $\left.\phi\right|_{[0,1 / 4]}=0,\left.\phi\right|_{[3 / 4,1]}=1$.

Suppose now that $\left(c_{0}, \omega_{0}, h_{0}\right) \sim\left(c_{1}, \omega_{1}, h_{1}\right)$ via $H_{0}, C_{0}$ and $\left(c_{1}, \omega_{1}, h_{1}\right) \sim$ $\left(c_{2}, \omega_{2}, h_{2}\right)$ via $H_{1}, C_{1}$ where the $H$ 's and $C$ 's have been replaced as above so that they are constant near the endpoints. We apply the above lemma to $[0,2] \times(M, N)$ with open cover $\left[0,1[\times(M, N),] \frac{3}{4}, \frac{5}{4}[\times(M, N)] 1,2,\right] \times(M, N)$ and the cochains $H_{0}, \operatorname{pr}^{*} h_{1}, H_{1}$ translated properly to obtain $H$. The cochain $H$ clearly restricts to $h_{0}$ and $h_{2}$ at the endpoints. Let $C$ be the composition of homotopies $C_{0}, C_{1}$. We have to verify the equation of cocycles

$$
\delta H=\operatorname{pr}^{*} \omega-C^{*} \iota_{n} .
$$

By Remark 3.7 it suffices to consider a smooth simplex $\sigma$ with image entirely contained in one of the three open subsets (because $\delta H(\sigma)=\delta H \circ \operatorname{sd}^{n}(\sigma)$ for any $n \in \mathbb{N})$. For example, if $\sigma\left(\Delta^{n}\right) \subset\left[0,1\left[\times M\right.\right.$ then $\sigma=i_{*} \sigma$ for the inclusion $i:[0,1[\times M \subset[0,2] \times M$. We have

$$
(\delta H)(\sigma)=\left(\delta i^{*} H\right)(\sigma)=\left(\delta H_{0}\right)(\sigma)=\operatorname{pr}^{*} \omega(\sigma)-\left(C_{0}^{*}\right) \iota_{n}(\sigma)
$$

which is also the value of $\operatorname{pr}^{*} \omega-C^{*} \iota_{n}$ on $\sigma=i_{*} \sigma$.

\subsection{Verification of the Axioms}

In Theorem 3.12 we will give an elementary verification of the fact that the differential cohomology groups of Definition 3.3 along with maps $(I, R, a)$, to be defined below in Definition 3.11, do indeed constitute a differential extension of $E$-cohomology (the group structure will be treated later).

It is clear that this verification on the elementary level must be somewhat technical in nature. We refer to Section 9 for a more elegant (but also more involved) treatment in terms of $\infty$-category theory.

Definition 3.9 (p.240 in [tD08]). The 'standard' Alexander-Whitney map is the natural chain equivalence

$$
A W: C_{n}(X \times Y) \rightarrow \bigoplus_{p+q=n} C_{i}(X) \otimes C_{j}(Y)
$$


which assigns to $\sigma: \Delta^{n} \rightarrow X \times Y$ the sequence in the direct sum

$$
\sum_{p+q=n} p\left(\operatorname{pr}_{1} \circ \sigma\right) \otimes\left(\operatorname{pr}_{2} \circ \sigma\right)_{q}
$$

Here, ${ }_{p} \tau\left(t_{0}, \ldots, t_{p}\right)=\tau\left(t_{0}, \ldots, t_{p}, 0 \ldots, 0\right)$ denotes the front $p$-face of $\tau$, and similarly $\tau_{q}$ is the back $q$-face with the zeros up front.

The standard Alexander-Whitney map has better formal properties than those that are obtained abstractly from the theory of Acyclic Models.

Lemma 3.10. For any $v \in C^{n-1}(X, A)$ there exists a cocycle $E \in Z^{n}(I \times$ $(X, A))$ such that $i_{0}^{*} E=0, i_{1}^{*} E=\delta v$. In particular, for differential cocycles

$$
h-h^{\prime} \in \operatorname{im}(\delta) \quad \Longrightarrow \quad[c, \omega, h]=\left[c, \omega, h^{\prime}\right] .
$$

Proof. Suppose first that $A=\emptyset$. Define the cochain $E$ as

$$
E: C_{n}(I \times X) \stackrel{\operatorname{pr} \circ A W}{\longrightarrow} C_{0}(I) \otimes C_{n}(X) \stackrel{\text { id } \otimes \partial}{\longrightarrow} C_{0}(I) \otimes C_{n-1}(X) \stackrel{\varepsilon \otimes v}{\longrightarrow} \mathbb{R},
$$

where

$$
\varepsilon\left(\sum_{x \in I} r_{x}\left[\Delta^{0} \rightarrow\{x\} \subset I\right]\right):=\sum_{x \in I} r_{x} \cdot x .
$$

From the formula for $A W$

$$
E\left(\Delta^{n} \stackrel{\left(\sigma_{1}, \sigma_{2}\right)}{\longrightarrow} I \times X\right)=\sigma_{1}(\overrightarrow{0}) \cdot v\left(\partial \sigma_{2}\right) .
$$

It follows that $i_{0}^{*} E=0, i_{1}^{*} E=\delta v$, and that $E$ is a cocycle. In the relative case, the cochain $E$ just constructed is zero on $C_{n}(I \times A)$, by naturality of $A W$. There is an analogous result for smooth cochains since $A W$ takes the complex of smooth cochains to itself. A version with coefficients in a graded vector space is deduced by applying our result to each component of the product (see Definition 2.5.

Definition 3.11. Define natural transformations

$$
\begin{aligned}
I: \hat{E}^{n}(M, N) & \rightarrow E^{n}(M, N), & & {[c, \omega, h] \mapsto[c], } \\
R: \hat{E}^{n}(M, N) & \rightarrow \Omega_{\mathrm{cl}}^{n}(M, N ; V), & & {[c, \omega, h] \mapsto \omega, } \\
a: \Omega^{n-1}(M, N ; V)_{d} & \rightarrow \hat{E}^{n}(M, N), & & \theta \mapsto\left[\text { const }_{\mathrm{pt} \in E_{n}}, d \theta, \theta\right] .
\end{aligned}
$$


Note that $a$ is well-defined since $\delta \theta=d \theta=d \theta-$ const $^{*} \iota_{n}$ (the fundamental cocycles are reduced). By (18), $a$ vanishes on $\operatorname{im}(d)$.

Recall that a sequence of maps between pointed sets

$$
A \stackrel{f}{\rightarrow} B \stackrel{g}{\rightarrow} C
$$

is said to be exact if $\operatorname{im}(f)=g^{-1}(\mathrm{pt})$. If $A, B, C$ are abelian groups, $f, g$ are homomorphisms, and the base-point is the zero element, then this implies that we have an exact sequence in the usual sense.

We will use slant products along $I$ of cochains from the left

$$
\int_{I}: C^{n+1}(I \times X) \rightarrow C^{n}(X),
$$

whose definition and elementary properties are reviewed in Remark 6.3. Recall that the singular cohomology and the deRham cohomology agree for manifolds. We obtain a transformation

$$
E^{n-1}(M, N) \stackrel{\mathrm{ch}}{\rightarrow} H_{\mathrm{dR}}^{n-1}(M, N ; V)=\frac{\Omega_{\mathrm{cl}}^{n-1}(M, N ; V)}{\operatorname{im}(d)} \rightarrow \frac{\Omega^{n-1}(M, N ; V)}{\operatorname{im}(d)}
$$

which is used in the following theorem:

Theorem 3.12. The following sequence is exact:

$E^{n-1}(M, N) \stackrel{\text { ch }}{\longrightarrow} \Omega^{n-1}(M, N ; V) / \operatorname{im}(d) \stackrel{a}{\longrightarrow} \hat{E}^{n}(M, N) \stackrel{I}{\longrightarrow} E^{n}(M, N) \longrightarrow 0$

Moreover, $R \circ a=d$ and cano $R=\operatorname{choI}$ (straightforward from the definitions).

Proof. We begin with two observations from the deRham isomorphism (7):

(i) Every smooth cocycle is cohomologous to a closed form.

(ii) If a closed form bounds a singular cochain it also bounds a form.

Exactness at $E^{n}(M, N): I$ is surjective by (i) applied to the cocycle $c^{*} \iota_{n}$.

Exactness at $\hat{E}^{n}(M, N)$ : Clearly $I \circ a=0$. Conversely, $I[c, \omega, h]=0$ yields a homotopy $C: c \simeq$ const rel $N$. Since also $C \simeq$ const rel $N$ we may pick $e \in C^{n-1}(I \times(M, N) ; V)$ with $\delta e=C^{*} \iota_{n}$. Consider

$$
u=h+i_{0}^{*} e-i_{1}^{*} e \in C_{s}^{n-1}(M, N ; V)
$$


for the inclusions $i_{0}, i_{1}:(M, N) \rightarrow I \times(M, N)$. Then $\delta u=\omega$ is a differential form so that by (ii) we may write $\delta u=d \kappa$ for some differential form $\kappa \in$ $\Omega^{n-1}(M, N ; V)$. By (i) we may then write

$$
u-\kappa=\eta+\delta v \quad \text { for } \quad \eta \in \Omega_{\mathrm{cl}}^{n-1}(M, N ; V), v \in C_{s}^{n-2}(M, N ; V) .
$$

Using Lemma 3.10 choose a cocycle $E \in Z_{s}^{n-1}(I \times(M, N) ; V)$ with $i_{0}^{*} E=$ $0, i_{1}^{*} E=\delta v$. Set

$$
H:=\operatorname{pr}_{2}^{*} h+\left(\operatorname{pr}_{2}^{*} i_{0}^{*}-\mathrm{id}\right) e-E \in C_{s}^{n-1}(I \times(M, N) ; V) \text { and } \theta=\kappa+\eta .
$$

By construction, the pair $(C, H)$ witnesses $(c, \omega, h) \sim($ const, $d \theta, \theta)=a(\theta)$.

Exactness at $\Omega^{n-1}(M, N ; V)_{d}$ : Suppose next that (const, 0,0$) \sim a(\theta)=$ (const, $d \theta, \theta)$, that is, we have $d \theta=0$ and are given $H \in C_{s}^{n-1}(I \times(M, N) ; V)$, $C:$ const $\simeq$ const $:(M, N) \rightarrow\left(E_{n}, \mathrm{pt}\right)$ with

$$
i_{0}^{*} H=\theta, i_{1}^{*} H=0, \quad \delta H=-C^{*} \iota_{n} .
$$

We have to show that $\theta$ is cohomologous to an element of type $c^{*} \iota_{n-1}$ for a map $c:(M, N) \rightarrow\left(E_{n-1}, \mathrm{pt}\right)$. Using the structure maps, let $c=\left(\varepsilon_{n-1}^{\text {adj }}\right)^{-1} \circ$ $C^{\text {adj }}$, so that we have $C=\varepsilon_{n-1} \circ(\Sigma c)$. We will write ' $\equiv$ ' for 'up to coboundary.' Using the compatibility of the fundamental cocycles (16) we have

$$
c^{*} \iota_{n-1}=c^{*} \int_{I} \varepsilon_{n-1}^{*} \iota_{n}=\int_{I} C^{*} \iota_{n}=-\int_{I} \delta H=\delta \int_{I} H+i_{0}^{*} H-i_{1}^{*} H \equiv \theta .
$$

To conclude the proof of exactness at $\Omega^{n-1}(M, N ; V)_{d}$ it remains to check $a \circ \operatorname{ch}[c]=0$. This may be concluded from the following proposition, applied to the homotopy $C:$ const $\simeq$ const, corresponding as above under the adjoint structure maps to $c:(M, N) \rightarrow\left(E_{n-1}, \mathrm{pt}\right)$.

It is our convention that homotopies $I \times X \rightarrow Y$ have the unit interval on the left, so in the following proposition the integral is taken from the left.

Proposition 3.13. Given a homotopy $C: c_{0} \simeq c_{1}($ rel $N)$ we obtain for all $\omega \in \Omega^{n}(M, N ; V), h_{0} \in C^{n-1}(M, N ; V)$ equivalences

$$
\left(c_{0}, \omega, h_{0}\right) \sim\left(c_{1}, \omega, h_{0}-\int_{I} C^{*} \iota_{n}\right) \quad \text { in } \quad \hat{E}^{n}(M, N)
$$


Proof. Set $h_{1}=h_{0}-\int_{I} C^{*} \iota_{n}$. We first remark that $\left(c_{1}, \omega, h_{1}\right)$ satisfies $\delta\left(h_{0}-\int_{I} C^{*} \iota_{n}\right)=\left(\omega-c_{0}^{*} \iota_{n}\right)+i_{0}^{*} C^{*} \iota_{n}-i_{1}^{*} C^{*} \iota_{n}=\omega-c_{1}^{*} \iota_{n}$. We seek a suitable $H \in C_{s}^{n-1}(I \times(M, N))$ with $i_{0}^{*} H=h_{0}, i_{1}^{*} H=h_{1}$ and $\delta H=\operatorname{pr}^{*} \omega-C^{*} \iota_{n}=$ $\delta \operatorname{pr}^{*} h_{1}+\left(\left(c_{1} \circ \mathrm{pr}\right)^{*}-C^{*}\right) \iota_{n}$. The homotopy $K: C \simeq\left(c_{1} \circ \operatorname{pr}\right)$

$$
K: I \times I \times(M, N) \rightarrow\left(E_{n}, \mathrm{pt}\right),(s, t, x) \mapsto \begin{cases}C(t, x) & (s \leq t), \\ C(s, x) & (s \geq t)\end{cases}
$$

is relative to $I \times N$ and yields a chain homotopy

$$
\delta \int_{I} K^{*} \iota_{n}=\left(i_{1}\right)^{*} K^{*} \iota_{n}-\left(i_{0}\right)^{*} K^{*} \iota_{n}=\left(c_{1} \circ \mathrm{pr}\right)^{*} \iota_{n}-C^{*} \iota_{n} .
$$

We may thus take $H=\operatorname{pr}^{*} h_{1}+\int_{I} K^{*} \iota_{n}$. Then

$$
\begin{aligned}
& \left(i_{0}\right)^{*} H=h_{1}+\int_{I}\left(\operatorname{id}_{I} \times i_{0}\right)^{*} K^{*} \iota_{n}=h_{1}+\int_{I} C^{*} \iota_{n}=h_{0}, \\
& \left(i_{1}\right)^{*} H=h_{1}+\int_{I}\left(\operatorname{id}_{I} \times i_{1}\right)^{*} K^{*} \iota_{n}=h_{1}+\int_{I}\left(c_{1} \circ \mathrm{pr}\right)^{*} \iota_{n} .
\end{aligned}
$$

The assertion now follows from Lemma 3.10 since $\int_{I}\left(c_{1} \circ \mathrm{pr}\right)^{*} \iota_{n}=\int_{I} \operatorname{pr}^{*}(\omega-$ $\left.\delta h_{1}\right)=\int_{I} \operatorname{pr}^{*} \omega-\int_{I} \operatorname{pr}^{*} \delta h_{1}=-\int_{I} \operatorname{pr}^{*} \delta h_{1}=\delta \int_{I} \operatorname{pr}^{*} h_{1}$ is a coboundary, using the fact that $\int_{I} \operatorname{pr}^{*} \omega=0$ for differential forms.

\subsection{Exact Sequence of Pairs}

Definition 3.14. The associated flat theory is defined as

$$
\hat{E}_{\text {flat }}^{*}(M, N):=\operatorname{ker}\left(\hat{E}^{*}(M, N) \stackrel{R}{\longrightarrow} \Omega_{\mathrm{cl}}^{*}(M, N)\right)
$$

In [HS05, (4.57)] it is shown that $\hat{E}_{\text {flat }}^{*}(M)$ may be canonically identified with $E \mathbb{R} / \mathbb{Z}^{*-1}(M)$. Applying the same reasoning to relative differential function complexes (using the obvious relative version of [HS05, Definition 4.1]) shows that $\hat{E}_{\text {flat }}^{*}(M, N)$ may be identified with $E \mathbb{R} / \mathbb{Z}^{*-1}(M, N)$. We will only need that $\hat{E}_{\text {flat }}^{*}$ is a cohomology theory, which is also independently shown in [BS10, Theorem 7.11]. 
Theorem 3.15. For any closed submanifold $N \subset M$ we have a natural long exact sequence

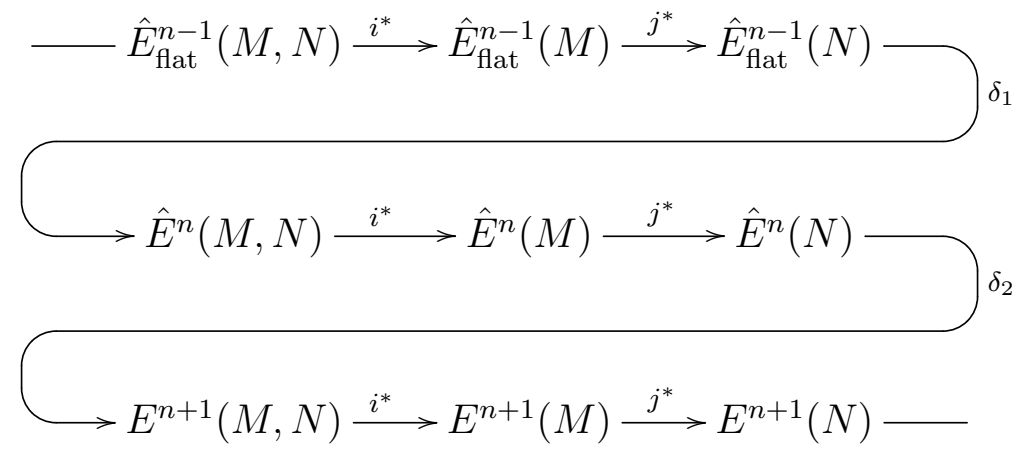

Here, the coboundary maps are defined as the compositions

$$
\begin{gathered}
\delta_{1}: \hat{E}_{\text {flat }}^{n-1}(N) \stackrel{\delta}{\longrightarrow} \hat{E}_{\text {flat }}^{n}(M, N) \stackrel{\subset}{\longrightarrow} \hat{E}^{n}(M, N) \\
\delta_{2}: \hat{E}^{n}(N) \stackrel{I}{\longrightarrow} E^{n}(N) \stackrel{\delta}{\longrightarrow} E^{n+1}(M, N) .
\end{gathered}
$$

Proof. Exactness at $\hat{E}^{n}(M): j^{*} \circ i^{*}=0$ follows directly by definition. Suppose conversely that $\left.(c, \omega, h)\right|_{N} \sim($ const, 0,0$)$. This means that there exists a homotopy $C:\left.c\right|_{N} \simeq$ const and cochain $H \in C_{s}^{n-1}(I \times N ; V)$ satisfying

$$
i_{0}^{*} H=\left.h\right|_{N}, i_{1}^{*} H=0, \quad j^{*} \omega=0, \quad \delta H=-C^{*} \iota_{n} .
$$

Pulling back along a smooth strictly increasing function $\phi:[0,1] \rightarrow[0,1]$ with $\phi_{[0,1 / 4]}=0, \phi_{3 / 4,1}=1$, we may assume that $\left.C\right|_{[0,1 / 4] \times N}=\left.c\right|_{N} \circ \mathrm{pr}$ and $\left.H\right|_{[0,1 / 4] \times N}=\left.\operatorname{pr}^{*} h\right|_{N}$ are 'constant near the endpoints.'

Applying Lemma 3.6 to the open subsets

$$
0 \times M \cup\left[0, \frac{1}{4}[\times N, \quad] 0,1\right] \times N
$$

and the cochains $\operatorname{pr}_{2}^{*} h, H$, we find $\tilde{H} \in C_{s}^{n-1}(0 \times M \cup I \times N)$ with

$$
\left.\tilde{H}\right|_{0 \times M}=h,\left.\tilde{H}\right|_{I \times N}=H .
$$

Because $0 \times N \subset 0 \times M \cup I \times N$ is closed we may define $\tilde{C}$ by gluing $C$ and $c$. As in the proof of Proposition 3.8, Remark 3.7 implies that $\delta \tilde{H}=\operatorname{pr}^{*} \omega-\tilde{C}^{*} \iota_{n}$. 
Since $N \subset M$ is a cofibration we may find a smooth retraction $5^{5}$

$$
r: I \times M \rightarrow(0 \times M) \cup(I \times N) .
$$

Pulling back along $r$ we obtain $\delta r^{*} \tilde{H}=\operatorname{pr}^{*} \omega-(\tilde{C} \circ r)^{*} \iota_{n}$, which witnesses an equivalence in $\hat{E}^{n}(M)$ from $(c, \omega, h)$ to $\left(\left.(\tilde{C} \circ r)\right|_{1 \times M}, \omega,\left.r^{*} \tilde{H}\right|_{1 \times M}\right)$, which represents an element of $\hat{E}^{n}(M, N)$.

Exactness at $\hat{E}^{n}(M, N)$ : If $i^{*}[c, \omega, h]=0$ then $\omega=0$ so that $[c, \omega, h] \in$ $\hat{E}_{\text {flat }}^{n}(M, N)$. By the exactness of the sequence of pairs of $\hat{E}_{\text {flat }}^{*}$ at $\hat{E}_{\text {flat }}^{n}(M, N)$ it follows that $[c, \omega, h]$ lies in the image of the coboundary operator $\delta$ : $\hat{E}_{\text {flat }}^{n-1}(N) \rightarrow \hat{E}_{\text {flat }}^{n}(M, N)$ which is also the image of $\delta_{1}$. It is also clear that $i^{*} \circ \delta_{1}=0$.

Exactness at $\hat{E}_{\text {flat }}^{n-1}(N)$ : Clearly $\delta_{1} \circ j^{*}=\operatorname{incl} \circ \delta \circ j^{*}=0$. Conversely, if $\delta_{1}(x)=0$ then $\delta(x)=0$. By the exactness of the sequence of pairs of $\hat{E}_{\text {flat }}^{*}$ it follows that $x$ lies in the image of $j^{*}$.

Exactness at $\hat{E}^{n}(N)$ : We have $\delta_{2} \circ j^{*}=0$ by the commutative diagram

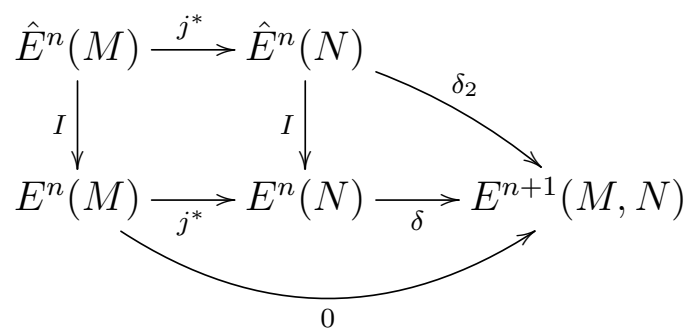

Suppose next that $\delta_{2}(\hat{x})=0$. Then $\delta(I(\hat{x}))=0$ so that $I(\hat{x})$ has a preimage $y \in E^{n}(M)$ under $j^{*}: E^{n}(M) \rightarrow E^{n}(N)$. By the surjectivity of $I$ we may write $y=I(\hat{y})$ and then $j^{*} \hat{y}-\hat{x} \in \operatorname{ker}(I)=\operatorname{im}(a)$ so that we may write $a(\theta)=j^{*} \hat{y}-\hat{x}$ by Theorem 3.12, $\theta \in \Omega^{n-1}(N ; V)$ may be extended by (6) to a differential form $\bar{\theta} \in \Omega^{n-1}(M ; V)$. Then

$$
j^{*}(\hat{y}-a(\bar{\theta}))=j^{*} \hat{y}-a(\theta)=\hat{x} .
$$

Exactness at $E^{n+1}(M, N)$ : Clearly $i^{*} \circ \delta_{2}=i^{*} \circ \delta \circ I=0$. Conversely, if $i^{*} x=0$ for $x \in E^{n+1}(M, N)$ then by the exact sequence of pairs of $E^{*}$ we

\footnotetext{
${ }^{5}$ Start with a continuous retraction and deform to a smooth map relative to the closed subset $0 \times M \cup I \times N$.
} 
may write $x=\delta(y)$ for $y \in E^{n}(N)$. Since $I$ is surjective, $y=I(\hat{y})$ for some $\hat{y} \in \hat{E}^{n}(N)$ and then $\delta_{2}(\hat{y})=x$.

\subsection{Addition (Rationally Even Case)}

The goal of this section is to quickly sketch how to construct the additive structure on the differential cohomology groups in an elementary manner. A similar method will be applied when we deal with products later. An explicit abelian group structure in the general case will be the topic of a later chapter. We will assume that $E$ is rationally even, which means that

$$
\pi_{2 n+1}(E) \otimes \mathbb{R}=0, \quad \forall n \in \mathbb{Z}
$$

This condition allows us to make arbitrary choices in even degrees. It is then our strategy to extend this data to odd degrees by a specific choice.

Using Theorem 2.3, select for $n$ even maps $\alpha_{n}: E_{n} \times E_{n} \rightarrow E_{n}$ representing addition in cohomology $E^{n}$. These are then extended to odd degrees by using the structure maps $E_{n-1} \approx \Omega E_{n}$. Since suspension is linear, $\alpha_{n-1}$ also represents addition. Because $\alpha_{n}$ represents addition and the Chern character is linear, $\operatorname{pr}_{1}^{*}\left[\iota_{n}\right]+\operatorname{pr}_{2}^{*}\left[\iota_{n}\right]=\alpha_{n}^{*}\left[\iota_{n}\right]$ in cohomology and we may therefore choose reduced cochains $A_{n} \in \tilde{C}^{n-1}\left(E_{n} \times E_{n} ; V\right)$ with

$$
\delta A_{n}=\operatorname{pr}_{1}^{*} \iota_{n}+\operatorname{pr}_{2}^{*} \iota_{n}-\alpha_{n}^{*} \iota_{n}
$$

Using $\varphi_{n}: \Sigma\left(E_{n} \times E_{n}\right) \rightarrow \Sigma E_{n} \times \Sigma E_{n} \stackrel{\varepsilon_{n} \times \varepsilon_{n}}{\longrightarrow} E_{n+1} \times E_{n+1}$ we make a specific choice for $A_{n-1}$ :

$$
A_{n-1}=-\int_{S^{1}} \varphi_{n-1}^{*} A_{n} \in \tilde{C}^{n-2}\left(E_{n-1} \times E_{n-1} ; V\right)
$$

The formula for $A_{n}$ implies a corresponding formula (20) for $A_{n-1}$. The addition is then defined as

$$
\left[c_{0}, \omega_{0}, h_{0}\right]+\left[c_{1}, \omega_{1}, h_{1}\right]=\left[\alpha_{n} \circ\left(c_{0}, c_{1}\right), \omega_{0}+\omega_{1}, h_{0}+h_{1}+\left(c_{0}, c_{1}\right)^{*} A_{n}\right] .
$$

This is well-defined: If $\left(C_{0}, H_{0}\right)$ witnesses an equivalence $x_{0}=\left(c_{0}, \omega_{0}, h_{0}\right) \sim$ $\left(c_{0}^{\prime}, \omega_{0}, h_{0}^{\prime}\right)=x_{0}^{\prime}$ and $\left(C_{1}, H_{1}\right)$ witnesses $x_{1}=\left(c_{1}, \omega_{1}, h_{1}\right) \sim\left(c_{1}^{\prime}, \omega_{1}, h_{1}^{\prime}\right)=x_{1}^{\prime}$ in the sense of Definition 3.3, then

$$
Y=\left(\alpha_{n} \circ\left(C_{0}, C_{1}\right), \operatorname{pr}^{*}\left(\omega_{0}+\omega_{1}\right), H_{0}+H_{1}+\left(C_{0}, C_{1}\right)^{*} A_{n}\right)
$$


relates the corresponding sums.

The abelian group axioms are then more or less automatic in even degrees. To prove the various equalities we need to construct equivalences as in Definition 3.3 between the involved triples. Using Lemma 3.10 and Proposition 3.13 , this ultimately boils down to proving that certain combinations of cochains differ only by a coboundary. Using 'rationally even,' this is easy in even degrees $n$ since it then suffices to prove that their difference is a cocycle. Our choice of $\alpha_{n-1}$ and $A_{n-1}$ then allows us to conclude the same for degree $n-1$, by constructing specific equivalences, using the structure maps and integration.

Consider for example associativity: we have to compare the differential cocycles

$\left[\alpha_{n}\left(\alpha_{n} \times \mathrm{id}\right)\left(c_{0}, c_{1}, c_{2}\right),\left(\omega_{0}+\omega_{1}\right)+\omega_{2}, h_{0}+h_{1}+\left(c_{0}, c_{1}\right)^{*} A_{n}+h_{2}+\left(\alpha_{n}\left(c_{0}, c_{1}\right), c_{2}\right)^{*} A_{n}\right]$, $\left[\alpha_{n}\left(\mathrm{id} \times \alpha_{n}\right)\left(c_{0}, c_{1}, c_{2}\right), \omega_{0}+\left(\omega_{1}+\omega_{2}\right), h_{0}+h_{1}+h_{2}+\left(c_{1}, c_{2}\right)^{*} A_{n}+\left(c_{0}, \alpha_{n}\left(c_{1}, c_{2}\right)\right)^{*} A_{n}\right]$.

Choose $H_{n}: \alpha_{n}\left(\alpha_{n} \times \mathrm{id}\right) \simeq \alpha_{n}\left(\mathrm{id} \times \alpha_{n}\right)$ for $n$ even and extend by requiring the formula $\varepsilon_{n-1} \circ \Sigma H_{n-1}=H_{n} \circ\left(\operatorname{id}_{I} \times \varepsilon_{n-1} \times \varepsilon_{n-1} \times \varepsilon_{n-1}\right)$. Using $(19)$ and (18) we see that it suffices to show that the pullback along $\left(c_{0}, c_{1}, c_{2}\right)$ of

$$
\operatorname{pr}_{12}^{*} A_{n}+\left(\alpha_{n} \times \mathrm{id}\right)^{*} A_{n}-\int_{I} H_{n}^{*} \iota_{n}-\operatorname{pr}_{23}^{*} A_{n}-\left(\mathrm{id} \times \alpha_{n}\right)^{*} A_{n}
$$

is a coboundary. Since $E$ is rationally even and $n$ is even, it suffices to show that it is a cocycle. Applying $\delta$ yields

$$
\begin{aligned}
& \operatorname{pr}_{12}^{*}\left(\operatorname{pr}_{1}^{*} \iota_{n}+\operatorname{pr}_{2}^{*} \iota_{n}-\alpha_{n}^{*} \iota_{n}\right)+\left(\alpha_{n} \times \mathrm{id}\right)^{*}\left(\operatorname{pr}_{1}^{*} \iota_{n}+\operatorname{pr}_{2}^{*} \iota_{n}-\alpha_{n}^{*} \iota_{n}\right)+\left(\alpha_{n} \times \mathrm{id}\right)^{*} \alpha_{n}^{*} \iota_{n} \\
& -\left(\mathrm{id} \times \alpha_{n}\right)^{*} \alpha_{n}^{*} \iota_{n}-\operatorname{pr}_{23}^{*}\left(\operatorname{pr}_{1}^{*} \iota_{n}+\operatorname{pr}_{2}^{*} \iota_{n}-\alpha_{n}^{*} \iota_{n}\right)-\left(\mathrm{id} \times \alpha_{n}\right)^{*}\left(\operatorname{pr}_{1}^{*} \iota_{n}+\operatorname{pr}_{2}^{*} \iota_{n}-\alpha_{n}^{*} \iota_{n}\right)
\end{aligned}
$$

which is zero. Let $\psi_{n-1}: \Sigma\left(E_{n-1} \times E_{n-1} \times E_{n-1}\right) \rightarrow E_{n} \times E_{n} \times E_{n}$ be the obvious map. Applying $-\int_{S^{1}} \psi_{n-1}^{*}$ to $\left(\nabla_{n}\right)$ we obtain the corresponding coboundary $\left(\diamond_{n-1}\right)$, by the way we have chosen everything in degree $n-1$.

Remark 3.16. Let $E$ be an $\Omega$-spectrum. We can make canonical choices

$$
\alpha_{n}: E_{n} \times E_{n} \approx \Omega E_{n+1} \times \Omega E_{n+1} \stackrel{\kappa}{\longrightarrow} \Omega E_{n+1} \approx E_{n}
$$

where the map $\kappa$ is concatenation, which is compatible with the integration of cochains up to explicit coboundary terms. For $e=\pi: \Delta^{2} \rightarrow$ 
$\Delta^{1},\left(t_{0}, t_{1}, t_{2}\right) \mapsto\left(t_{0}+t_{1} / 2, t_{2}+t_{1} / 2\right)$ we have, denoting by $[a, b]$ the 1 -simplex $\Delta^{1} \rightarrow[a, b],(x, y) \mapsto y a+x b$, the relation $\partial e=\left[0, \frac{1}{2}\right]-[0,1]+\left[\frac{1}{2}, 1\right]$ so that

$$
\int_{[0,1]} u=\int_{\left[0, \frac{1}{2}\right]} u+\int_{\left[\frac{1}{2}, 1\right]} u-\int_{\partial e} u=\int_{\left[0, \frac{1}{2}\right]} u+\int_{\left[\frac{1}{2}, 1\right]} u+\left((-1)^{|e|} \delta \int_{e} u-\int_{e} \delta u\right) .
$$

This may be used to show that $\alpha_{n}^{*} \iota_{n}$ and $\operatorname{pr}_{1}^{*} \iota_{n}+\operatorname{pr}_{2}^{*} \iota_{n}$ differ by the coboundary of the canonical cochain

$$
A_{n}=\left(\varepsilon_{n}^{\mathrm{adj}} \times \varepsilon_{n}^{\mathrm{adj}}\right)^{*} \int_{e}\left(\kappa^{\mathrm{adj}}\right)^{*} \iota_{n+1} .
$$

This observation can be used to exhibit an abelian group structure in the general case. One can then construct 'by hand' higher simplices that exhibit the coherence conditions.

The idea of the previous remark will be dealt with more systematically in the following two chapters.

\section{Fundamental Groupoids of Cospectra}

It is well-known that infinite loop spaces possess a rich algebraic structure, they are all algebras for the little $\infty$-cubes operad. In this section, we shall extract parts of this algebraic structure, adapted to our context. While none of the results here should come as a surprise, they are much less trivial to establish than one might expect (especially for spectra of simplicial sets and if the adjoint structure maps are not isomorphisms). This is reflected for instance by the fact that we are only able to construct an equivalence class of lifts of the fundamental groupoid functor (29). In particular, we lack a canonical choice.

\subsection{Cospectra and the Category 2BrMonCat}

A cospectrum is a spectrum object in the opposite of the category of spaces. Everything in this chapter goes through for spectra as well, but it will turn out later that it is more convenient for us to regard the spaces we are interested in as spectra whose adjoint structure maps are looking in the 'wrong' direction. 
In this section we will freely use the theory of simplicial sets, whose basic notions are recalled in A.3. For the more involved parts of the theory we will refer to the appropriate places in [GJ09].

Definition 4.1. A cospectrum is a sequence of pointed Kan complexes $E_{n}$, $n \in \mathbb{Z}$, which are equipped with pointed 'structure' maps (weak equivalences)

$$
\Omega E_{n+1} \stackrel{\sim}{\longrightarrow} E_{n} .
$$

Example 4.2. Every spectrum $E=\left(E_{n}, \varepsilon_{n}\right)$ whose adjoint structure maps $\varepsilon_{n}^{\text {adj }}: E_{n} \rightarrow \Omega E_{n+1}$ are isomorphisms may be regarded as a cospectrum. More generally, suppose that all $\varepsilon_{n}^{\text {adj }}$ are trivial Kan fibrations. By choosing sections, $E$ may be viewed as a cospectrum.

Definition 4.3. Cospectra are the objects of a category coSpt. Morphisms $f: E \rightarrow F$ are sequences of pointed maps $f_{n}: E_{n} \rightarrow F_{n}$ which commute strictly with the maps (21).

The goal of this section is to construct, for each $n$, a lift of the functor 'fundamental groupoid at level $n$,'

$$
\left(\Pi_{1}\right)_{-n}: \text { coSpt } \rightarrow \text { Cat }, \quad E \mapsto \Pi_{1} E_{n},
$$

along the forgetful functor to the following category:

Definition 4.4. The category $2 \mathrm{BrMonCat}$ has as objects (small) categories $\mathcal{C}$ with two braided monoidal structures ' $(\mathbb{D}$ ', ' $\ominus$ ', sharing a unit, and a braided monoidal structure on the identity functor $e:(\mathcal{C}, \mathbb{D}) \rightarrow(\mathcal{C}, \ominus)$. As morphisms we take functors $F: \mathcal{C} \rightarrow \mathcal{D}$ which are braided monoidal for both structures and which induce a commutative diagram in MonCat

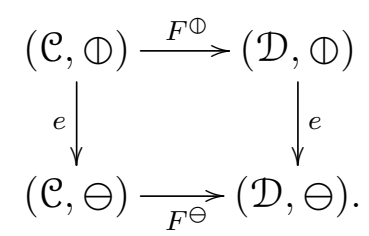

We will call the isomorphisms $e_{x, y}: x \oplus y \rightarrow x \ominus y$ encoded in the monoidal structure on the functor e the Eckmann-Hilton isomorphisms. 
A morphism $F:(\mathcal{C},(\mathbb{D}, \ominus, e) \rightarrow(\mathcal{D}, \oplus, \ominus, e)$ in $\mathbf{2 B r M o n C a t}$ is said to be strict if the two braided monoidal structures on the functors

$$
F^{\oplus}:(\mathcal{C}, \oplus) \rightarrow(\mathcal{D}, \oplus), \quad F^{\ominus}:(\mathcal{C}, \ominus) \rightarrow(\mathcal{D}, \ominus)
$$

are strict.

The elementary theory of (braided) monoidal categories, functors, and transformations is outlined in A.1. A number of non-standard results on the behaviour of 'monoidal notions' under equivalences are developed in A.2.

Remark 4.5. There is a forgetful functor from the category of 'tensor categories with strict braided multiplications' as studied in [JS93, p.56] to 2BrMonCat. In fact, the objects of $2 \mathrm{BrMonCat}$ we shall use arise in this way (see the discussion following Definition 4.9). Objects of 2BrMonCat are not what are traditionally called 2-monoidal categories.

Example 4.6. Any braided monoidal category is an object of $2 \mathrm{BrMonCat}$ in which $(1)=\ominus$ and $e=\mathrm{id}$.

The following example will be studied in more rigor in the next subsection:

Example 4.7. Suppose $X$ is a pointed topological space. Let

$$
\Omega X=(X, \mathrm{pt})^{\left(S^{1}, 1\right)}=\left\{\gamma: S^{1} \rightarrow X \text { continuous } \mid \gamma(1)=\mathrm{pt}\right\}
$$

denote the loop space of $X$ (with the compact open topology [DK01, p.113]). The one-fold loop space $\left(\Pi_{1} \Omega^{1}(\Omega X), \Phi\right)$ is easily seen to give rise to a monoidal category. Also, $M(f, g)=f \ominus g$ is a (strict) multiplication on $\left(\Pi_{1} \Omega^{2} X, \ominus\right)$ in the sense of [JS93] on account of the identity

$$
(A \ominus B) \oplus(C \ominus D)=(A \oplus C) \ominus(B \oplus D) .
$$

Here we view $M$ as a strict monoidal functor and the transformations $\rho_{f}$ : $M(f$, const $) \simeq f, \lambda_{f}: M$ (const, $\left.f\right) \simeq f$ are given by the obvious homotopies. This implies $\Pi_{1} \Omega^{2} X \in 2$ BrMonCat. Pictorially: 


$$
\begin{aligned}
& f(1) g=\begin{array}{|l|l|}
\hline f & g
\end{array} \quad f \ominus g=\mid \begin{array}{|l|}
\hline g \\
\hline
\end{array}
\end{aligned}
$$

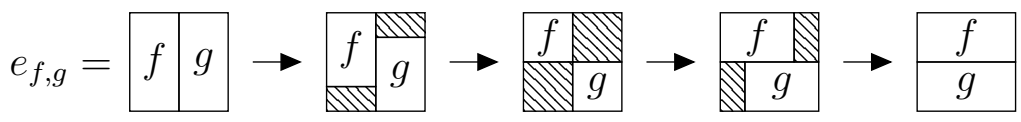

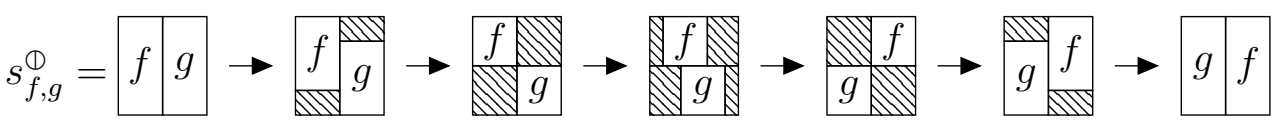

\subsubsection{Fundamental Groupoids of Double Loop Spaces}

The two monoidal structures on the fundamental groupoid $\Omega^{2} X$ of a double loop space of a pointed topological space $(X, \mathrm{pt})$ are given as follows:

$$
\begin{aligned}
& (f \oplus g)(s, t)= \begin{cases}f(2 s, t) & (0 \leq s \leq 1 / 2), \\
g(2 s-1, t) & (1 / 2 \leq s \leq 1) .\end{cases} \\
& (f \ominus g)(s, t)= \begin{cases}f(s, 2 t) & (0 \leq t \leq 1 / 2), \\
g(s, 2 t-1) & (1 / 2 \leq t \leq 1) .\end{cases}
\end{aligned}
$$

The unit object $1 \in \Pi_{1} \Omega^{2} X$ in both cases is given by the constant loop. Let us consider ' $(1$ ' in detail; the structure ' $\ominus$ ' in analogous by replacing the roles of $s$ and $t$. Clearly, a map $X \rightarrow Y$ induces a strict monoidal functor $\Pi_{1} \Omega^{2} X \rightarrow \Pi_{1} \Omega^{2} Y$ for each of the monoidal structures ' $\Phi$ ', ' $\ominus$ '.

Proposition 4.8. $\left(\Pi_{1} \Omega^{2} X, \Phi, 1, a^{\Phi}, \lambda^{\Phi}, \rho^{\Phi}\right)$ is a monoidal category (the isomorphims $a^{\Phi}, \lambda^{\Phi}, \rho^{\Phi}$ are defined below).

Proof. We begin by turning ' $\Phi$ ' into a functor $\Pi_{1} \Omega^{2} X \times \Pi_{1} \Omega^{2} X \rightarrow \Pi_{1} \Omega^{2} X$. Write $F \circ G$ for the composition of homotopies

$$
(F \circ G)(s, t, u)= \begin{cases}G(s, t, 2 u) & (0 \leq u \leq 1 / 2) \\ F(s, t, 2 u-1) & (1 / 2 \leq u \leq 1)\end{cases}
$$


For $f \in \Omega^{2} X, \operatorname{id}_{f}(s, t, u)=f(s, t)$ denotes the constant homotopy. If $F$ : $f_{1} \simeq f_{2}$ and $G: g_{1} \simeq g_{2}$ are homotopies, we set

$$
(F \oplus G)(s, t, u)= \begin{cases}F(2 s, t, u) & (0 \leq s \leq 1 / 2), \\ G(2 s-1, t, u) & (1 / 2 \leq s \leq 1)\end{cases}
$$

These definitions turn ' $\left(\right.$ ' into a functor, on behalf of the equalities $\left(F \circ F^{\prime}\right)$ (1) $\left(G \circ G^{\prime}\right)=(F \oplus G) \circ\left(F^{\prime} \oplus G^{\prime}\right)$ and $\operatorname{id}_{f} \oplus \mathrm{id}_{g}=\mathrm{id}_{f \Phi g}$. For a monoidal structure, we also need to provide associativity and unit constraints $a^{\Phi}, \lambda^{\Phi}, \rho^{\Phi}$. Set

$$
a_{f, g, h}^{\Phi}= \begin{cases}f\left(\frac{4 s}{1+u}, t\right) & \left(0 \leq s \leq \frac{1+u}{4}\right), \\ g(4 s-1-u, t) & \left(\frac{1+u}{4} \leq s \leq \frac{2+u}{4}\right), \\ h\left(\frac{4 s}{2-u}-\frac{2+u}{2-u}, t\right) & \left(\frac{2+u}{4} \leq s \leq 1\right) .\end{cases}
$$

The verification that $a$ is a natural transformation is based on the following observation:

Claim: A continuous map $h:[0,1] \times[0,1] \rightarrow X$ induces a homotopy $h(1,-) \circ$ $h(-, 0) \simeq h(-, 1) \circ h(0,-)$.

Proof. We can take the homotopy

$$
(t, u) \mapsto \begin{cases}h(2 t, 0) & (0 \leq t \leq 1 / 2,0 \leq u \leq 1 / 2-t) \\ h(t-u+1 / 2, t+u-1 / 2) & (0 \leq t \leq 1 / 2,1 / 2-t \leq u \leq 1 / 2+t), \\ h(0,2 t) & (0 \leq t \leq 1 / 2,1 / 2+t \leq u \leq 1) \\ h(1,2 t-1) & (1 / 2 \leq t \leq 1,0 \leq u \leq t-1 / 2) \\ h(t-u+1 / 2, t+u-1 / 2) & (1 / 2 \leq t \leq 1, t-1 / 2 \leq u \leq 3 / 2-t), \\ h(2 t-1,1) & (1 / 2 \leq t \leq 1,3 / 2-t \leq u \leq 1) .\end{cases}
$$

We need to check that $a_{f_{2}, g_{2}, h_{2}}^{\oplus} \circ[(F \oplus G) \oplus H]$ is homotopic to $[F \oplus(G \oplus$ $H)] \circ a_{f_{1}, g_{1}, h_{1}}^{\Phi}$. This follows by applying the claim (using $u, v$ ) to the map

$$
(s, t, u, v) \mapsto \begin{cases}F\left(\frac{4 s}{1+u}, t, v\right) & \left(0 \leq s \leq \frac{1+u}{4}\right) \\ G(4 s-1-u, t, v) & \left(\frac{1+u}{4} \leq s \leq \frac{2+u}{4}\right) \\ H\left(\frac{4 s}{2-u}-\frac{2+u}{2-u}, t, v\right) & \left(\frac{2+u}{4} \leq s \leq 1\right)\end{cases}
$$


The unit constraints are given by the following formulas:

$$
\begin{aligned}
& \lambda_{f}^{\Phi}: 1 \oplus f \rightarrow f, \quad \lambda_{f}^{\Phi}(s, t, u)= \begin{cases}\mathrm{pt} & \left(0 \leq s \leq \frac{1-u}{2}\right), \\
f\left(\frac{2 s}{1+u}-\frac{1-u}{1+u}, t\right) & \left(\frac{1-u}{2} \leq s \leq 1\right),\end{cases} \\
& \rho_{f}^{\Phi}: f \Phi 1 \rightarrow f, \quad \rho_{f}^{\Phi}(s, t, u)= \begin{cases}f\left(\frac{2 s}{1+u}, t\right) & \left(0 \leq s \leq \frac{1+u}{2}\right), \\
\mathrm{pt} & \left(\frac{1+u}{2} \leq s \leq 1\right)\end{cases}
\end{aligned}
$$

The triangle equality $\rho_{f \Phi g} \simeq\left(\operatorname{id}_{f} \oplus \rho_{g}\right) \circ a_{f, g, 1}$ is exhibited by the homotopy

$$
(s, t, u, v) \mapsto \begin{cases}\mathrm{pt} & \left(0 \leq u \leq 1, \frac{u+1}{2} \leq s \leq 1\right), \\ f\left(\frac{4 s}{u+u v+1}, t\right) & \left(0 \leq u \leq \frac{1}{2}, 0 \leq s \leq \frac{u+u v+1}{4}\right), \\ g\left(\frac{4 s}{u-u v+1}-\frac{u+u v+1}{u-u v+1}, t\right) & \left(0 \leq u \leq \frac{1}{2}, \frac{u+u v+1}{4} \leq s \leq \frac{u+1}{2}\right), \\ f\left(\frac{4 s}{u+v-u v+1}, t\right) & \left(\frac{1}{2} \leq u \leq 1,0 \leq s \leq \frac{u+v-u v+1}{4}\right), \\ g\left(\frac{4 s}{u-v+u v+1}-\frac{u+v-u v+1}{u-v+u v+1}, t\right) & \left(\frac{1}{2} \leq u \leq 1, \frac{u+v-u v+1}{4} \leq s \leq \frac{u+1}{2}\right) .\end{cases}
$$

The other triangle equality is analogous. The commutativity of Mac Lane's Pentagon can also shown by linear reparameterization: both

$$
a_{f, g, h \Phi i} \circ a_{f \Phi g, h, i}, \quad\left(1_{f} \oplus a_{g, h, i}\right) \circ a_{f, g \oplus h, i} \circ\left(a_{f, g, h} \oplus 1_{i}\right)
$$

are homotopic to the homotopy

$$
b(s, t, u)= \begin{cases}f\left(\frac{8 s}{1+3 u}, t\right) & \left(0 \leq s \leq \frac{1+3 u}{8}\right) \\ g\left(\frac{8 s}{1+u}-\frac{1+3 u}{1+u}, t\right) & \left(\frac{1+3 u}{8} \leq s \leq \frac{1+2 u}{4}\right) \\ h\left(\frac{8 s}{2-u}-\frac{2+4 u}{2-u}, t\right) & \left(\frac{1+2 u}{4} \leq s \leq \frac{4+3 u}{8}\right) \\ i\left(\frac{8 s}{4-3 u}-\frac{4+3 u}{4-u}, t\right) & \left(\frac{4+3 u}{8} \leq s \leq 1\right)\end{cases}
$$

For example, a homotopy $a_{f, g, h \bowtie i} \circ a_{f \oplus g, h, i} \simeq b$ is given by

$$
(s, t, u, v) \mapsto \begin{cases}f\left(\frac{8 s}{1+3 u+u v}, t\right) & \left(0 \leq u \leq \frac{1}{2}, 0 \leq s \leq \frac{1+3 u+u v}{8}\right), \\ g\left(\frac{8 s}{1+u-u v}-\frac{1+3 u+u v}{1+u-u v}, t\right) & \left(0 \leq u \leq \frac{1}{2}, \frac{1+3 u+u v}{8} \leq s \leq \frac{1+2 u}{4}\right), \\ h\left(\frac{8 s}{2-v u}-\frac{2+4 u}{2-v u}, t\right) & \left(0 \leq u \leq \frac{1}{2}, \frac{1+2 u}{4} \leq s \leq \frac{4+4 u-v u}{8}\right), \\ i\left(\frac{8 s}{4-4 u+v u}-\frac{4+4 u-v u}{4-4 u+v u}\right) & \left(0 \leq u \leq \frac{1}{2}, \frac{4+4 u-v u}{8} \leq s \leq 1\right), \\ f\left(\frac{8 s}{v-u v+4 u}, t\right) & \left(\frac{1}{2} \leq u \leq 1,0 \leq s \leq \frac{v-u v+4 u}{8}\right), \\ g\left(\frac{8 s}{2-v+u v}-\frac{v-u v+4 u}{2-v+u v}, t\right) & \left(\frac{1}{2} \leq u \leq 1, \frac{v-u v+4 u}{8} \leq s \leq \frac{1+2 u}{4}\right), \\ h\left(\frac{8 s}{5-v+2 u+u v}-\frac{2+4 u}{5-v+2 u+u v}\right) & \left(\frac{1}{2} \leq u \leq 1, \frac{1+2 u}{4} \leq s \leq \frac{5-v+2 u+u v}{8}\right), \\ i\left(\frac{8 s}{3+v-2 u-u v}-\frac{5-v+2 u+u v}{3+v-2 u-u v}\right) & \left(\frac{1}{2} \leq u \leq 1, \frac{5-v+2 u+u v}{8} \leq s \leq 1\right) .\end{cases}
$$

The other homotopy is similar and has twelve cases. 
The monoidal structure ' $\ominus$ ' is obtained by replacing the roles of the variables $s$ and $t$. To establish the monoidal structure on the identity functor (the 'Eckmann-Hilton isomorphisms') it is difficult to proceed directly: instead, we will use the following proposition, due to [JS93] (see also [AM10, Proposition 6.11]). Definition 4.9 is a special case of [AM10, Proposition 6.4], where we assume that the units $I=J$ strictly coincide and that $\Delta_{I}=\mu_{J}=\mathrm{id}$.

Definition 4.9. A strong 2-monoidal category (sharing units) consists of a category $\mathcal{C}$, two monoidal structures $\left(\oplus, I, a^{\oplus}, \lambda^{\oplus}, \rho^{\oplus}\right),\left(\ominus, I, a^{\ominus}, \lambda^{\ominus}, \rho^{\ominus}\right)$ on $\mathcal{C}$, sharing a unit $I$ with $I \oplus I=I, I \ominus I=I$, and a natural isomorphism

$$
\zeta_{A, B, C, D}:(A \ominus B) \oplus(C \ominus D) \rightarrow(A \oplus C) \ominus(B \oplus D)
$$

The maps 23) are required to endow both functors

$$
\text { (1) }(\mathcal{C} \times \mathcal{C}, \ominus) \rightarrow(\mathcal{C}, \ominus), \quad \ominus:(\mathcal{C} \times \mathcal{C}, \Phi) \rightarrow(\mathcal{C}, \oplus)
$$

with the structure of strong monoidal functors.

Proposition 6.11 in [AM10]. A strong 2-monoidal category (sharing units) $\mathcal{C}$ gives rise to braidings on each of ' $\mathbb{1}$ ', ' $\ominus$ '. The identity functor can be viewed as a braided strong monoidal isomorphism $e^{\mathbb{D}, \ominus}:(\mathcal{C}, \mathbb{(}) \rightarrow(\mathcal{C}, \ominus)$ (the monoidal structure is defined in terms of the interchange isomorphism).

This braid on ' $\Phi$ ' is given by the composition

$$
\begin{aligned}
& a \oplus b \stackrel{\lambda^{\ominus} \oplus \rho^{\ominus}}{\longleftarrow}(1 \ominus a) \oplus(b \ominus 1) \cong(1 \oplus b) \ominus(a \oplus 1) \stackrel{\lambda^{\oplus} \ominus \rho^{\oplus}}{\longrightarrow} b \ominus a \\
& \stackrel{\rho^{\oplus} \ominus \lambda^{\oplus}}{\longleftarrow}(b \oplus 1) \ominus(1 \oplus a) \cong(b \ominus 1) \oplus(1 \ominus a) \stackrel{\lambda^{\ominus} \oplus \rho^{\ominus}}{\longrightarrow} b \oplus a .
\end{aligned}
$$

The strong monoidal structure $e^{\Phi, \ominus}$ on the identity functor will be called Eckmann-Hilton isomorphisms for the structures ' $\Phi$ ', ' $\ominus$ ' because they are given by the familiar composition

$$
e_{a, b}^{\Phi, \ominus}: a \oplus b \stackrel{\rho^{\ominus} \oplus \lambda^{\ominus}}{\longleftarrow}(a \ominus I) \oplus(I \ominus b) \cong(a \oplus I) \ominus(I \oplus b) \stackrel{\rho^{\oplus} \ominus \lambda^{\oplus}}{\longrightarrow} a \ominus b .
$$

The unit constraint $e_{I}^{\oplus, \ominus}: I \rightarrow I$ is given by the identity morphism. 
Consider the category $\Pi_{1}\left(\Omega^{2} X\right)$ with the two monoidal structures ' $($ '), ' $\ominus$ ' defined above. Then we even have an equality

$$
(f \ominus g) \oplus(h \ominus i)=(f \oplus h) \ominus(g \oplus i) .
$$

By the way we have defined the associativity and unitality constraints (not using the $t$ variable) we have identities

$$
\begin{aligned}
a_{A, A^{\prime}, A^{\prime \prime}}^{\Phi} \ominus a_{B, B^{\prime}, B^{\prime \prime}}^{\Phi} & =a_{A \ominus B, A^{\prime} \ominus B^{\prime}, A^{\prime \prime} \ominus B^{\prime \prime}}^{\Phi}, \\
\rho_{A \ominus B}^{\Phi} & =\rho_{A}^{\Phi} \ominus \rho_{B}^{\Phi}, \\
\lambda_{A \ominus B}^{\Phi} & =\lambda_{A}^{\Phi} \ominus \lambda_{B}^{\Phi} .
\end{aligned}
$$

This is just the assertion that ' $\ominus$ ' in (24) is a strict monoidal functor. By changing the roles of $s$ and $t$ we get dual identities which show that ' $(\mathbb{})$ in (24) is also strict monoidal. The two monoidal structures on $\Pi_{1}\left(\Omega^{2} X\right)$ therefore satisfy the conditions of [AM10, Proposition 6.11]. We conclude that $\Pi_{1}\left(\Omega^{2} X\right)$ has two canonical braided monoidal structures (given by the compositions $(*)$ above) and canonically the structure of braided monoidal functor on the identity between these two monoidal structures. Spelling out the definition, we have explicitly

$$
e_{f, g}^{\Phi, \ominus}(s, t, u)= \begin{cases}f\left(2 s, \frac{t}{1-u}\right) & \left(u \leq \frac{1}{2}, s \leq \frac{1}{2}, t \leq 1-u\right), \\ \mathrm{pt} & \left(u \leq \frac{1}{2}, s \leq \frac{1}{2}, t \geq 1-u\right), \\ \mathrm{pt} & \left(u \leq \frac{1}{2}, s \geq \frac{1}{2}, t \leq u\right), \\ g\left(2 s-1, \frac{t}{1-u}-\frac{u}{1-u}\right) & \left(u \leq \frac{1}{2}, s \geq \frac{1}{2}, t \geq u\right), \\ f\left(\frac{s}{u}, 2 t\right) & \left(u \geq \frac{1}{2}, t \leq \frac{1}{2}, s \leq u\right), \\ \mathrm{pt} & \left(u \geq \frac{1}{2}, t \leq \frac{1}{2}, s \geq u\right), \\ \mathrm{pt} & \left(u \geq \frac{1}{2}, t \geq \frac{1}{2}, s \leq 1-u\right), \\ g\left(\frac{s}{u}-\frac{1-u}{u}, 2 t-1\right) & \left(u \geq \frac{1}{2}, t \geq \frac{1}{2}, s \geq 1-u\right) .\end{cases}
$$

The definition makes no explicit use of the space $X$ so that a map $X \rightarrow Y$ clearly preserves the Eckmann-Hilton isomorphisms. To conclude:

Proposition 4.10. For each pointed space $X, \Pi_{1} \Omega^{2} X$ may be viewed as an object of 2BrMonCat. A pointed map $X \rightarrow Y$ induces a strict morphism $\Pi_{1} \Omega^{2} X \rightarrow \Pi_{1} \Omega^{2} Y$ in 2BrMonCat. 


\subsection{Fundamental Groupoids of Loop Spaces}

\subsubsection{Fundamental Groupoid of a Kan complex}

Definition 4.11. Let $K \in$ Set $_{\Delta}$ be a Kan complex. The fundamental groupoid $\Pi_{1} K$ has object set $K_{0}$. A morphism from $k \in K_{0}$ to $k^{\prime} \in K_{0}$ is a homotopy class $[\mathrm{u}]\left(\operatorname{rel} \partial \Delta^{1}\right)$ of paths $u: \Delta^{1} \rightarrow K$ from $k$ to $k^{\prime}$. Composition $[u] \circ[v]$ is given by choosing an arbitrary filler in the diagram

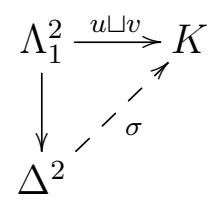

and passing to the homotopy class of $d_{1} \sigma$.

The following is an elementary, though important observation:

$\left(^{*}\right)$ Suppose $a, a^{\prime}: A \rightarrow X$ are homotopic rel $C, X$ fibrant. If $i: A \subset B$ is a trivial cofibration and $b, b^{\prime}: B \rightarrow X$ are extensions of $a, a^{\prime}$ along $i$, then $b$ and $b^{\prime}$ are homotopic rel $C$ as well.

Proof. Use the standard cylinder object $A \times \partial \Delta^{1} \rightarrow A \times \Delta^{1} \rightarrow A$ in Set $_{\Delta}$. Since $X$ is a Kan complex $a \simeq a^{\prime}$ implies that we may find a homotopy $h: A \times \Delta^{1} \rightarrow X$. By [GJ09, I.4.6] the map $A \times \Delta^{1} \cup B \times \partial \Delta^{1} \rightarrow B \times \Delta^{1}$ is again a trivial cofibration so that we may extend $h$ along it.

We conclude from $(*)$ that composition determined via $(26)$ is well-defined. It is associative and has identity morphisms $s_{0} k$ for every $k \in K_{0}$ (cf. GJ09, Section I.8]). The groupoid $\Pi_{1} K$ is equivalent to the more traditional 'topological' fundamental groupoid $\Pi_{1}|K|$ of the topological space $|K|$ by GJ09, Theorem III.1.1]. The equivalence is given by taking the geometric realization of points $k: \Delta^{0} \rightarrow K$ and paths $u: \Delta^{1} \rightarrow K$.

\subsubsection{Loop Spaces}

The loop space $\Omega K$ of a pointed Kan complex $K$ is the simplicial set whose

$n$-simplices are given by pointed simplicial maps (here, $L_{+}:=L \sqcup \Delta^{0}$ denotes the simplicial set $L$ together with a disjoint base point)

$$
f: S^{1} \wedge \Delta_{+}^{\bullet} \rightarrow K
$$


This is again a Kan complex. A pointed simplicial map $K \rightarrow L$ induces a map $\Omega K \rightarrow \Omega L$ of the loop spaces by post-composition. That is, $\Omega$ is a functor. We wish to lift the composition of the functors

$$
\mathrm{Kan}^{*} \stackrel{\Omega}{\longrightarrow} \mathrm{Kan}^{*} \stackrel{\Pi_{1}}{\longrightarrow} \text { Cat }
$$

to a functor ' $\Pi_{1} \Omega$ ' taking values in monoidal categories.

Recall that we have a pair of adjoint functors (even Quillen adjunction), which relate the theory of simplicial sets and topological spaces (see GJ09, Theorem I.11.4]):

$$
|\cdot|: \text { CGHaus } \rightleftarrows \text { Set }_{\Delta}: \text { sing }
$$

For any pointed Kan complex $K$, geometric realization induces an equivalence

$$
|\cdot|: \Pi_{1} \Omega K \stackrel{\simeq}{\longrightarrow} \Pi_{1} \Omega|K| .
$$

In more detail, for any pointed simplicial set $K$ there is a canonical map (inducing 27 by applying $\Pi_{1}$ )

$$
\Omega K \rightarrow \operatorname{sing} \Omega|K|,
$$

which takes an simplex $S^{1} \wedge \Delta_{+}^{\bullet} \rightarrow K$ to the adjoint of the geometric realization $\left|S^{1}\right| \wedge\left|\Delta_{+}^{\bullet}\right| \rightarrow|K|$, that is, the pointed map $\left|\Delta_{+}^{\bullet}\right| \rightarrow|K|^{\left|S^{1}\right|}$, which amounts to an unpointed map $\Delta^{\bullet} \rightarrow|K|^{\left|S^{1}\right|}$. For pointed Kan complexes $K$ the map $\left(^{*}\right)$ is a homotopy equivalence [HS05, Section 4.6], by the following computation of homotopy groups

$$
\begin{aligned}
\pi_{i} \Omega K & \cong \pi_{i+1} K, \\
\pi_{i} \operatorname{sing} \Omega|K| & \cong \pi_{i} \Omega|K| \cong \pi_{i+1}|K| \cong \pi_{i+1} K .
\end{aligned}
$$

For later use, we record also that, due to the Quillen equivalence $|\cdot| \dashv$ sing, the adjoint map

$$
|\Omega K| \rightarrow \Omega|K|
$$

is a weak equivalence for every pointed Kan complex $K$.

Of course, $\Pi_{1} \Omega|K|$ has a canonical monoidal structure ' $\Phi$ ' determined by the composition of loops. According to Proposition A.9 we may choose a 
monoidal structure ' $\left(1\right.$ ' on $\Pi_{1} \Omega K$ so that (27) becomes a monoidal equivalence. To make $\Pi_{1} \Omega$ a functor we need to define a monoidal structure on $\Pi_{1} \Omega f$ for each map $f: K \rightarrow L$ :

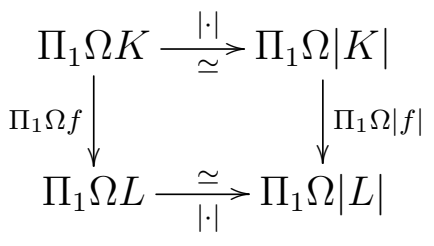

Proposition A.14 implies that there is a unique monoidal structure on $\Pi_{1} \Omega f$ so as to refine this diagram to MonCat. Uniqueness of course implies functoriality:

Proposition 4.12. There exists a functor $\Pi_{1} \Omega:$ Kan $^{*} \rightarrow$ MonCat into monoidal categories with $p \circ \Pi_{1} \Omega=\Pi_{1} \circ \Omega$, where $p:$ MonCat $\rightarrow$ Cat denotes the forgetful functor. For each $K \in \mathbf{K a n}^{*}$, geometric realization induces a monoidal functor

$$
\left(\Pi_{1} \Omega K,(1) \rightarrow\left(\Pi_{1} \Omega|K|, \oplus\right)\right.
$$

These are natural in $K$.

\subsubsection{Aside: Exhibition of Monoidal Structure}

Unlike the case of spaces, the fundamental groupoid of a loop space of a Kan complex does not carry a canonical monoidal structure. Using (27), we have singled out above an equivalence class of monoidal structures.

In this subsection we will give another view of this equivalence class, more intrinsic to simplicial sets. It may safely be skipped.

The objects of $\Pi_{1} \Omega K$ are based loops $u: S^{1} \rightarrow K$. A morphism $h: u \rightarrow v$ is (a homotopy class of) a homotopy of based loops $h: S^{1} \times \Delta^{1} \rightarrow K$ which restricts on $\partial \Delta^{1}$ to $u \sqcup v$. We now define a monoidal structure on $\Pi_{1} \Omega K$. For any pair of objects $u, v \in \Pi_{1} \Omega K$ choose fillers

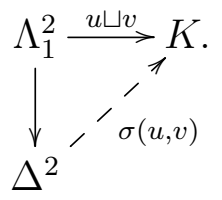


We set $u \otimes v=d_{1} \sigma(u, v)$ on objects. Suppose $[h]: u_{1} \rightarrow u_{2}$ and $[g]: v_{1} \rightarrow v_{2}$ are morphisms in $\Pi_{1} \Omega K$. Then we may choose a filler in the diagram

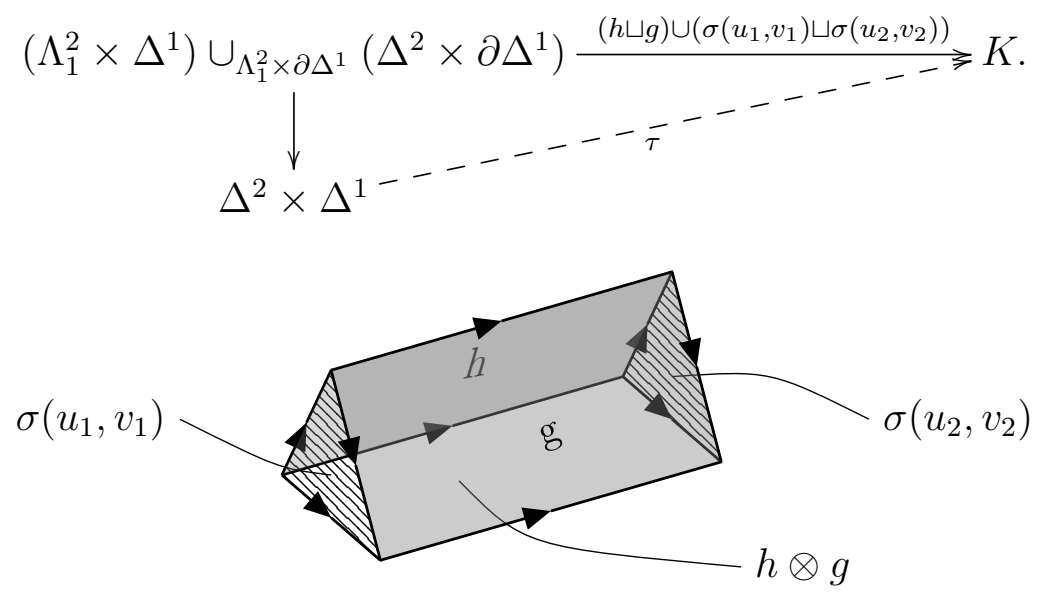

Define $h \otimes g$ as the homotopy class of $\tau \mid \Delta^{\{0,2\}} \times \Delta^{1}$. Using $\left(^{*}\right)$, this homotopy class is seen to be independent of the choice of representatives $h, g$ and of the choice of filler $\tau$.

Remark 4.13. The similarity between the definition of ' $\otimes$ ' and ' $O$ ' is no coincidence: $\Pi_{1} \Omega K$ can be identified with the automorphism category (at the base-point) of the fundamental 2-groupoid of $K$. Under this correspondence, the tensor product corresponds to vertical composition.

Lemma 4.14. We have defined a functor $\otimes: \Pi_{1} \Omega K \times \Pi_{1} \Omega K \rightarrow \Pi_{1} \Omega K$.

Proof. We must check $\left(h_{1} \otimes g_{1}\right) \circ\left(h_{2} \otimes g_{2}\right)=\left(h_{1} \circ h_{2}\right) \otimes\left(g_{1} \circ g_{2}\right)$. Choose a filler $\kappa$ in the diagram

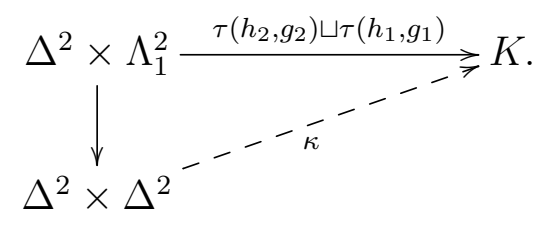

Then $\kappa \mid \Delta^{\{0,2\}} \times \Delta^{2}$ is a filler as in the definition of composition of $\kappa \mid \Delta^{\{0,2\}} \times$ $\Delta^{\{0,1\}}$ and $\kappa \mid \Delta^{\{0,2\}} \times \Delta^{\{1,2\}}$, whence $\kappa \mid \Delta^{\{0,2\}} \times \Delta^{\{0,2\}}$ represents the class of $\left(h_{1} \otimes g_{1}\right) \circ\left(h_{2} \otimes g_{2}\right)$. Also, $\kappa\left|\Delta^{\{0,1\}} \times \Delta^{2}, \kappa\right| \Delta^{\{1,2\}} \times \Delta^{2}$ are valid choices for fillers 
in the diagram defining composition in $\Pi_{1} \Omega K$. The classes of $\kappa \mid \Delta^{\{0,1\}} \times \Delta^{\{0,2\}}$ and $\kappa \mid \Delta^{\{1,2\}} \times \Delta^{\{0,2\}}$ are therefore $h_{1} \circ h_{2}, g_{1} \circ g_{2}$. The restriction $\kappa \mid \Delta^{2} \times \Delta^{\{0,2\}}$ shows that $\kappa \mid \Delta^{\{0,2\}} \times \Delta^{\{0,2\}}$ is the class of $\left(h_{1} \circ h_{2}\right) \otimes\left(g_{1} \circ g_{2}\right)$.

Next, we have to check that $\operatorname{id}_{u} \otimes \mathrm{id}_{u}=\operatorname{id}_{u}$ for every $u: S^{1} \rightarrow K$. This follows since we may choose the filler $\tau: \Delta^{2} \times \Delta^{1} \rightarrow K$ defining the tensor product as a degenerate simplex (pieced together by the degenerate simplices $\left.u: \Delta^{3} \rightarrow \Delta^{0} \rightarrow K\right)$.

To prove that this really defines a monoidal structure on $\Pi_{1} \Omega K$, we will take a shortcut that appeals to the readers knowledge that $\Pi_{1} \Omega|K|$ carries a monoidal structure. At the same time, this will show that the monoidal structure on $\Pi_{1} \Omega K$ is independent (up to monoidal equivalence) of the choice of fillers $\sigma(u, v)$ above. Note that we 'keep track' of $\sigma(u, v)$.

Recall that the monoidal structure on the fundamental groupoid $\Pi_{1} \Omega|K|$ may be defined similarly to the above, except that one takes a 'standard' choice of filler, coming from a standard retraction $\left|\Delta^{2}\right| \rightarrow\left|\Lambda_{1}^{2}\right|$ :

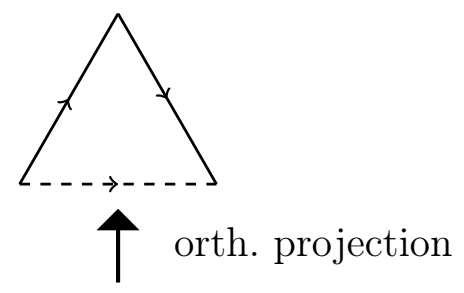

This amounts to the usual placing of loops and homotopies 'next to each other.' The associators and unitors are defined by the 'standard' homotopies, analogous to those used for the fundamental group.

Proposition 4.15. The functor $\otimes$ determines a monoidal structure on $\Pi_{1} \Omega K$. Different choices of fillers $\sigma(u, v)$ lead to equivalent monoidal structures. For each choice, the functor induced by geometric realization

$$
\Pi_{1} \Omega K \rightarrow \Pi_{1} \Omega|K|
$$

is a monoidal equivalence.

Proof. This follows from the proof of Proposition A.9. It suffices to remark that by $(*)$ the geometric realization of any choice of filler $\sigma(u, v)$ is homotopic 
to the standard choice in $\Pi_{1} \Omega|K|$. Restricting to the bottom face we obtain a homotopy $|u \otimes v| \simeq|u| \otimes|v|$ which, in the notation of the proof of A.9 is 'a' tensor product for $|u|$ and $|v|$ in $\Pi_{1} \Omega K$.

\subsubsection{Double Loop Spaces}

We have a forgetful functor

$$
q: \text { BBrMonCat } \rightarrow \text { MonCat }, \quad(\mathcal{C}, \oplus, \ominus, e) \mapsto(\mathcal{C}, \oplus)
$$

Proposition 4.16. The composition $\Pi_{1} \Omega \circ \Omega:$ Kan $^{*} \rightarrow$ MonCat may be lifted along $q$ to a functor $\Pi_{1} \Omega^{2}: \mathbf{K a n}^{*} \rightarrow \mathbf{2 B r M o n C a t}$ is such a way that for each $K \in \mathbf{K a n}^{*}$ 'geometric realization'

$$
\left(\Pi_{1} \Omega^{2} K, \oplus, \ominus, e\right) \rightarrow\left(\Pi_{1} \Omega^{2}|K|, \oplus, \ominus, e\right)
$$

is a morphism in 2BrMonCat.

Proof. The composition $\Pi_{1} \Omega \circ \Omega$ may be lifted to BrMonCat: we have a diagram of monoidal equivalences

$$
\left(\Pi_{1} \Omega\right)(\Omega K) \stackrel{\simeq}{\longrightarrow} \Pi_{1} \Omega|\Omega K| \stackrel{\simeq}{\longrightarrow} \Pi_{1} \Omega \Omega|K|
$$

The first functor is by definition of the monoidal structure on $\left(\Pi_{1} \Omega\right)(\Omega K)$ and is given by geometric realization. The second is induced by the canonical $\operatorname{map}|\Omega K| \rightarrow \Omega|K|$ and is obviously monoidal.

The category on the right of $(28)$ is braided monoidal. Using Proposition A.10, we obtain canonical braided monoidal structures on each of the categories and functors in $(28)$. Since $\Pi_{1} \Omega^{2}|f|$ is braided monoidal, Proposition A.11 implies that the functors $\left(\Pi_{1} \Omega\right)(\Omega f), \Pi_{1} \Omega|\Omega f|$ induced by a simplicial map $f$ are automatically braided.

According to Proposition 4.10, $\Pi_{1} \Omega^{2}|K| \in$ 2BrMonCat. Using Proposition A.9 we see that we may transport the second braided monoidal structure ' $\ominus$ ' to $\Pi_{1} \Omega^{2} K$ as well. The functoriality of the second structure is again determined by Proposition A.14. Also, the identity functor may be viewed uniquely as a monoidal functor $\left(\Pi_{1} \Omega^{2} K, \oplus\right) \rightarrow\left(\Pi_{1} \Omega^{2} K, \ominus\right)$, on account of Proposition A.14. This concludes the proof of Proposition 4.16. 
Remark 4.17. It is our convention that ' $($ ') denotes the monoidal structure on the fundamental groupoid of $\Omega^{2} K$ which comes by viewing it as the onefold loop space of $\Omega K$. The symbol ' $\Phi$ ' indicates 'horizontal concatenation.'

\subsection{Construction of Cospectrum Groupoids}

We now come to the construction of the functors

$$
\left(\Pi_{1}\right)_{-n}: \text { coSpt } \rightarrow \text { 2BrMonCat, } \quad n \in \mathbb{Z},
$$

which lift the assignments $E_{n} \mapsto \Pi_{1} E_{n}$.

\subsubsection{Definition on Objects}

For each object $E \in \mathbf{c o S p t}$ we have weak equivalences

$$
\Omega^{2} E_{n+2} \rightarrow \Omega^{1} E_{n+1} \rightarrow E_{n} .
$$

Let 2BrMonCat $\stackrel{q}{\rightarrow}$ MonCat $\stackrel{p}{\rightarrow}$ Cat denote the forgetful functors. We have a diagram of equivalences of categories

$$
p q \Pi_{1} \Omega^{2}\left(E_{n+2}\right) \rightarrow p \Pi_{1} \Omega\left(E_{n+1}\right) \rightarrow \Pi_{1} E_{n} .
$$

According to Proposition A.9 we may choose a monoidal structure '(1)' on $\Pi_{1} E_{n}$ so as to make

$$
\Pi_{1} \Omega\left(E_{n+1}\right) \rightarrow\left(\Pi_{1} E_{n}, \oplus\right)
$$

a monoidal equivalence. Also, $q \Pi_{1} \Omega^{2}\left(E_{n+2}\right)=\left(\Pi_{1} \Omega\right)\left(\Omega E_{n+2}\right) \rightarrow\left(\Pi_{1} \Omega\right)\left(E_{n+1}\right)$ is monoidal (since $\Pi_{1} \Omega$ is a functor). Therefore,

$$
q \Pi_{1} \Omega^{2}\left(E_{n+2}\right) \rightarrow \Pi_{1} \Omega\left(E_{n+1}\right) \rightarrow\left(\Pi_{1} E_{n},(\mathbb{D})\right.
$$

is a diagram of monoidal equivalences. The category on the left is braided, so Proposition A.10 implies that we may choose braids on the other two categories so as to refine (31) to BrMonCat.

With help of Propositions A.9, A.10 the second braided monoidal structure ' $\ominus$ ' on $\Pi_{1} \Omega^{2}\left(E_{n+2}\right)$ is analogously transported (involving choices of ' $\ominus$ ' and of the braids) to $\Pi_{1} \Omega\left(E_{n+1}\right)$ and then to $\Pi_{1} E_{n}$, so we have another diagram in BrMonCat

$$
\left(\Pi_{1} \Omega^{2}\left(E_{n+2}\right), \ominus\right) \rightarrow\left(\Pi_{1} \Omega\left(E_{n+1}\right), \ominus\right) \rightarrow\left(\Pi_{1} E_{n}, \ominus\right) .
$$


Consider now the following diagram in MonCat (the monoidal structure $e$ on the identity functor on the left is provided by Proposition 4.16):

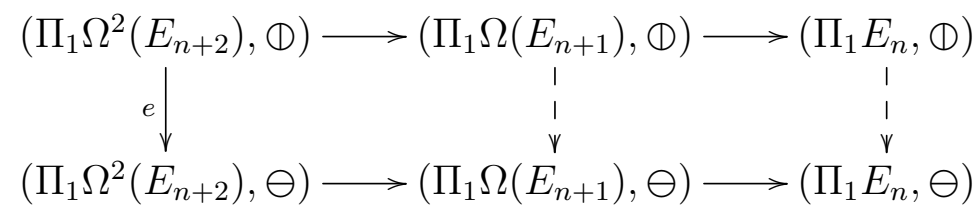

Using Proposition A.13, the dashed identity functors uniquely inherit monoidal structures, making the diagram commute. All in all, we have constructed a diagram

$$
\Pi_{1} \Omega^{2}\left(E_{n+2}\right) \rightarrow\left(\Pi_{1} \Omega\left(E_{n+1}\right), \ominus, e\right) \rightarrow\left(\Pi_{1} E_{n}, \oplus, \ominus, e\right) .
$$

in 2BrMonCat. We now set $\left(\Pi_{1}\right)_{-n} E=\left(\Pi_{1} E_{n}, \oplus, \ominus, e\right)$. This completes the definition of $(29)$ on objects.

\subsubsection{Cospectrum Groupoids for Abelian Group Spectra}

For a spectrum of simplicial abelian groups, the above construction may be greatly simplified: take both monoidal structures to be induced by the addition in the abelian group and the trivial Eckmann-Hilton isomorphisms. We prove below in (32) that this construction may be canonically identified with the one given above.

If $A$ is topological abelian group, the fundamental groupoid has a strict symmetric monoidal structure given by addition:

$$
a_{1} \otimes a_{2}:=a_{1}+a_{2}, \quad\left(a_{1} \stackrel{f}{\rightarrow} b_{1}\right) \otimes\left(a_{2} \stackrel{g}{\rightarrow} b_{2}\right):=f+g
$$

The loop space of a topological abelian group is again a topological abelian group, by pointwise addition of functions. The geometric realization $|A|$ of a simplicial abelian group $A_{\bullet}$ is a topological abelian group (induced by $|A| \times|A|=|A \times A| \rightarrow|A|)$.

Lemma 4.18. Suppose $X$ is a topological abelian group, base-pointed by 0. Then there is a canonical morphism in 2BrMonCat

$$
\left(\Pi_{1} \Omega^{2} X, \oplus, \ominus, e^{\Phi, \ominus}\right) \rightarrow\left(\Pi_{1} \Omega^{2} X,+,+, \mathrm{id}\right)
$$

covering the identity functor in Cat. 
Proof. We need to turn the identity functor into a monoidal functor in two ways:

$$
\left(\Pi_{1} \Omega^{2} X, \oplus\right) \rightarrow\left(\Pi_{1} \Omega^{2} X,+\right), \quad\left(\Pi_{1} \Omega^{2} X, \ominus\right) \rightarrow\left(\Pi_{1} \Omega^{2} X,+\right)
$$

This will be done by an Eckmann-Hilton argument, that is, by appealing to Proposition 6.11 in AM10] (recalled above): the equality

$$
(f+g) \oplus(h+i)=(f \oplus h)+(g \oplus i)
$$

along with the identities

$$
a_{f, f^{\prime}, f^{\prime \prime}}^{\Phi}+a_{g, g^{\prime}, g^{\prime \prime}}^{\Phi}=a_{f+g, f^{\prime}+g^{\prime}, f^{\prime \prime}+g^{\prime \prime}}^{\Phi}, \quad \rho_{f+g}^{\Phi}=\rho_{f}^{\Phi}+\rho_{g}^{\Phi}, \quad \lambda_{f+g}^{\Phi}=\lambda_{f}^{\Phi}+\lambda_{g}^{\Phi}
$$

yields the structure $e^{\mathbb{\Phi},+}$ of braided monoidal functor on id. Unravelling the definition of the Eckmann-Hilton isomorphism, we have

$$
e_{f, g}^{\Phi,+}(s, t, u)= \begin{cases}f\left(\frac{2 s}{1+u}, t\right) & \left(s \leq \frac{1-u}{2}\right), \\ f\left(\frac{2 s}{1+u}, t\right)+g\left(\frac{2 s}{1+u}-\frac{1-u}{1+u}, t\right) & \left(\frac{1-u}{2} \leq s \leq \frac{1+u}{2}\right), \\ g\left(\frac{2 s}{1+u}-\frac{1-u}{1+u}, t\right) & \left(s \geq \frac{1+u}{2}\right) .\end{cases}
$$

Exchanging the roles of $s$ and $t$ we obtain a similar Eckmann-Hilton homotopy for the other monoidal structure:

$$
e_{f, g}^{\ominus,+}(s, t, u)= \begin{cases}f\left(s, \frac{2 t}{1+u}\right) & \left(t \leq \frac{1-u}{2}\right), \\ f\left(s, \frac{2 t}{1+u}\right)+g\left(s, \frac{2 t}{1+u}-\frac{1-u}{1+u}\right) & \left(\frac{1-u}{2} \leq t \leq \frac{1+u}{2}\right), \\ g\left(s, \frac{2 t}{1+u}-\frac{1-u}{1+u}\right) & \left(t \geq \frac{1+u}{2}\right)\end{cases}
$$

Recall that from a morphism in 2BrMonCat we require that it be compatible with the Eckmann-Hilton isomorphisms on the target and source category. We have taken 'id' on the target category, so we must show $e^{\ominus,+} \circ e^{\Phi, \ominus}=e^{\Phi,+}$ as morphisms in $\Pi_{1} \Omega^{2} X$. We have:

$$
e_{f, g}^{\ominus,+} \circ e_{f, g}^{\oplus, \ominus}(s, t, u)= \begin{cases}f\left(2 s, \frac{t}{1-2 u}\right) & \left(u \leq \frac{1}{4}, s \leq \frac{1}{2}, t \leq 1-2 u\right), \\ g\left(2 s-1, \frac{t}{1-2 u}-\frac{2 u}{1-2 u}\right) & \left(u \leq \frac{1}{4}, s \geq \frac{1}{2}, t \geq 2 u\right), \\ f\left(\frac{s}{2 u}, 2 t\right) & \left(\frac{1}{4} \leq u \leq \frac{1}{2}, s \leq 2 u, t \leq \frac{1}{2}\right), \\ g\left(\frac{s}{2 u}-\frac{1-2 u}{2 u}, 2 t-1\right) & \left(\frac{1}{4} \leq u \leq \frac{1}{2}, s \geq 1-2 u, t \geq \frac{1}{2}\right), \\ f\left(s, \frac{t}{u}\right) & \left(u \geq \frac{1}{2}, t \leq 1-u\right), \\ f\left(s, \frac{t}{u}\right)+g\left(s, \frac{t}{u}-\frac{1-u}{u}\right) & \left(u \geq \frac{1}{2}, 1-u \leq t \leq u\right), \\ g\left(s, \frac{t}{u}-\frac{1-u}{u}\right) & \left(u \geq \frac{1}{2}, u \leq t\right), \\ 0 & (\text { else }) .\end{cases}
$$


A homotopy $e^{\ominus,+} \circ e^{\Phi, \ominus} \simeq e^{\Phi,+}$ is given by $H_{1}+H_{2}$, where

$$
\begin{aligned}
H_{1}(s, t, u, v)= \begin{cases}f\left(\frac{2 s}{1+u v}, \frac{t}{1-2 u+2 u v}\right) & \left(u \leq \frac{1}{4}, s \leq \frac{1+u v}{2}, t \leq 1-2 u+2 u v\right), \\
f\left(\frac{2 s}{v+4 u-3 u v}, \frac{2 t}{1+v}\right) & \left(\frac{1}{4} \leq u \leq \frac{1}{2}, s \leq \frac{v+4 u-3 u v}{2}, t \leq \frac{1+v}{2}\right), \\
f\left(\frac{2 s}{2-v+v u}, \frac{t}{u+v-u v}\right) & \left(\frac{1}{2} \leq u, s \leq \frac{2-v+v u}{2}, t \leq u+v-u v\right), \\
0 & (\text { else }),\end{cases} \\
H_{2}(s, t, u, v)= \begin{cases}g\left(1-\frac{2-2 s}{1+u v}, 1-\frac{1-t}{1-2 u+2 v}\right) & \left(u \leq \frac{1}{4}, s \geq \frac{1-u v}{2}, t \geq 2 u-2 u v\right), \\
g\left(1-\frac{2-2 s}{v+4 u-3 u v}, 1-\frac{2-2 t}{1+v}\right) & \left(\frac{1}{4} \leq u \leq \frac{1}{2}, s \geq \frac{2-v-4 u+3 u v}{2}, t \geq \frac{1-v}{2}\right), \\
g\left(1-\frac{2-2 s}{2-v+v u}, 1-\frac{1-t}{u+v-u v}\right) & \left(\frac{1}{2} \leq u, s \geq \frac{v-v u}{2}, t \geq 1-u-v+u v\right), \\
0 & (\text { else }) .\end{cases}
\end{aligned}
$$

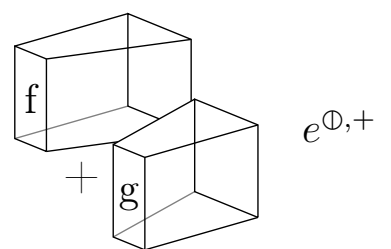

Remark 4.19. We have just defined a functor $\left(\Pi_{1}\right)_{-n}: \operatorname{coSpt} \rightarrow 2 \mathrm{BrMonCat}$. For cospectra $\left(E_{n}, \varepsilon_{n}\right)$ of simplicial abelian groups (with linear structure maps) there is another such functor

$$
\left(\Pi_{1}^{a b}\right)_{-n}: \operatorname{coSpt}^{a b} \rightarrow \text { 2BrMonCat }, \quad\left(E_{n}, \varepsilon_{n}\right) \mapsto\left(E_{n},+,+, \mathrm{id}\right) .
$$

We shall now describe a canonical natural isomorphism

$$
\left(\Pi_{1}\right)_{-n} \cong\left(\Pi_{1}^{a b}\right)_{-n}
$$

covering the identity isomorphism between the corresponding Cat-valued functors. When understood that we are working with a spectrum $E$ of simplicial abelian groups, we will identify $\left(\Pi_{1}\right)_{-n} E$ with the easier construction $\left(\Pi_{1}^{a b}\right)_{-n} E$ using this natural isomorphism.

By Lemma 4.18, there is a canonical way to view the identity as a morphism in 2BrMonCat

$$
\left(\Pi_{1} \Omega^{2}\left|E_{n+2}\right|, \oplus, \ominus, e^{\Phi, \ominus}\right) \rightarrow\left(\Pi_{1} \Omega^{2}\left|E_{n+2}\right|,+,+, \mathrm{id}\right) .
$$

By definition of the structure on $\left(\Pi_{1}\right)_{-n} E$, the first row in the following diagram consists of morphisms in 2BrMonCat. The second row is obviously 
by morphisms in $\mathbf{2 B r M o n C a t}$ and the left vertical morphism has just been endowed with such a structure:

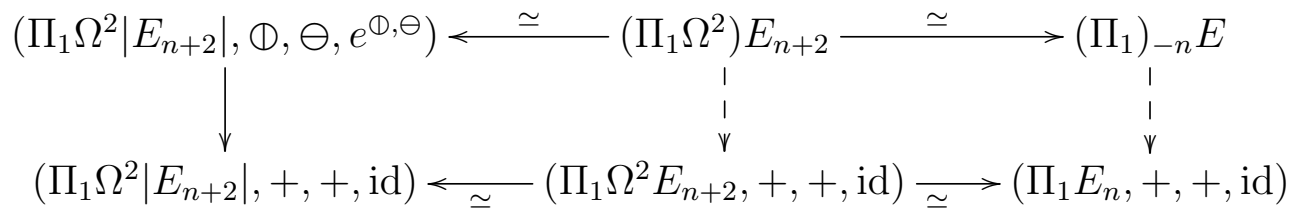

By Propositions A.11, A.13, and A.14 the dotted arrow may uniquely be endowed with the structure of morphism in 2BrMonCat, making the diagram commute in 2BrMonCat. The right vertical isomorphism yields the sought-for (32); naturality follows from uniqueness.

\subsubsection{Definition on Morphisms}

Suppose next that $f: E \rightarrow F$ is a map of cospectra. Since $\Pi_{1} \Omega^{2}(f)$ is a morphism in 2BrMonCat we may use Propositions A.13, A.11 to uniquely endow $\Pi_{1}(f)$ and $\Pi_{1}(\Omega f)$ with the structure of a monoidal functor, for each of the two structures:

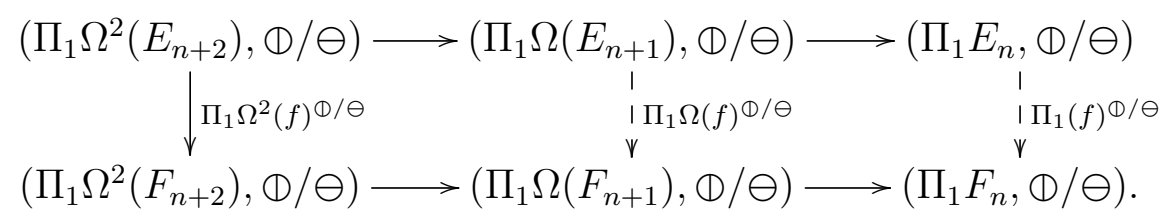

The uniqueness of the monoidal data implies that the assignments $f \mapsto$ $\Pi_{1} f^{\Phi / \ominus}, \Pi_{1} \Omega f^{\Phi / \ominus}$ are functorial also in this data. The commutativity of $\Pi_{1} f$ with the Eckmann-Hilton isomorphisms (namely 22 commutes) follows from the uniqueness part of Proposition A.13 and since this is true for $\Pi_{1} \Omega^{2} f$ (by Proposition 4.16).

\section{Additive Structures}

For our construction $\hat{E}$ above to qualify as a differential extension of $E$ in the sense of Definition 1.12 we must produce abelian group structures on the sets constructed in Definition 3.3 and prove that $a, I, R$ are homomorphisms.

We have already mentioned that the construction of additional structure on $\hat{E}$ relies on a coherent choice of the fundamental cocycles. The choice (16) 
is well-suited for defining integration maps.

For the additive structure it is possible to proceed in several ways:

1. Restrict to rationally even cohomology theories $E$. This was sketched in Section 3.5.

2. View $\hat{E}^{n}(M)$ as a homotopy group of an appropriate spectrum that depends on $M$. This will be the method of choice in the more advanced approach presented later. The main drawback is that it is hard to 'see' how addition works concretely. As such, it is difficult to relate it with other explicitly constructed structures.

3. Take a middle ground between 1 . and 2 . by observing that in an $\Omega$ spectrum (whose adjoint structure maps are homeomorphisms) there is a canonical choice for the maps $\alpha_{n}: E_{n} \times E_{n} \rightarrow E_{n}$ representing addition:

$$
E_{n} \times E_{n} \approx \Omega E_{n+1} \times \Omega E_{n+1} \stackrel{\text { concat }}{\longrightarrow} \Omega E_{n+1} \approx E_{n} .
$$

In this case, elements $A_{n}$ with 20 may be written down concretely (Remark 3.16) and the coherence conditions are exhibited by certain higher dimensional 'canonical' simplices. We will use the results of the previous chapter to proceed more systematically.

\subsection{The Cocycle Spectrum}

The goal of this section is to construct a spectrum whose $n$-th space is given by (singular) cocycles on the cosimplicial space $K \wedge \Delta_{+}^{\bullet}$ :

$$
\tilde{Z}^{n}\left(K \wedge \Delta_{+}^{\bullet}\right)
$$

The spaces (34) have been introduced in [HS05]. The arguments presented there imply that there exists a spectrum whose $n$-th level is weakly equivalent to (34). In part this is because they need to chose certain functorial sections which, strictly speaking, involves a fibrant replacement in a diagram model category (in particular, the spaces (34) are replaced).

We will give a refined treatment which produces functorial spectra with the actual spaces (34). This additional control will be crucial in verifying linearity of explicit constructions of further data defined on $\hat{E}$. 


\subsubsection{Reduced Chain Complex of a Space}

The forgetful functor $s \mathbf{A b} \rightarrow$ Set $_{\Delta}^{*}$ has a left adjoint $\tilde{\mathbb{Z}}$ given on a pointed simplicial set $K \in$ Set $_{\Delta}^{*}$ by the formula

$$
\tilde{\mathbb{Z}} K_{n}=\text { free abelian group on generators } K_{n} \backslash\{*\}=\mathbb{Z} K_{n} / \mathbb{Z}\{*\} .
$$

Composing it with the Moore complex functor $C: s \mathbf{A b} \rightarrow \mathbf{C h}_{\geq 0}$ into the category of positive chain complexes yields the reduced chain complex functor

$$
\tilde{C}: \mathbf{S e t}_{\Delta}^{*} \rightarrow \mathbf{C h}_{\geq 0} .
$$

Being the composition of two left adjoints, $\tilde{C}$ preserves colimits.

For a pointed topological space $X$, we will use the functor sing from (108) to extract a pointed simplicial set $K=\operatorname{sing}(X)$. We will also use the notation $\tilde{C}^{*}(X)=\tilde{C}^{*}(K)=C(\tilde{\mathbb{Z}} \operatorname{sing}(X))$.

\subsubsection{Alexander-Whitney and Eilenberg-Zilber Maps}

For each $p+q=n \in \mathbb{N}$ define morphisms ${ }_{p} \theta:[p] \rightarrow[n], \theta_{q}:[q] \rightarrow[n]$ in $\Delta$ by ${ }_{p} \theta(i)=i, \theta_{q}(i)=i+p$.

Definition 5.1. The Alexander-Whitney map is a canonical quasi-isomorphism

$$
\begin{array}{ll}
A W: C(A \otimes B) \stackrel{\sim}{\longrightarrow} C(A) \otimes C(B), & A, B \in s \mathbf{A b} \\
A W(a \otimes b)=\sum_{p+q=n}\left({ }_{p} \theta\right)^{*} a \otimes\left(\theta_{q}\right)^{*} b, & a \in A_{n}, b \in B_{n}
\end{array}
$$

from the complex of the level-wise tensor product of simplicial abelian groups to the tensor product of the corresponding chain complexes.

A shuffle $\lambda \in \Sigma(p, q)$ is a morphism $\lambda:[p+q] \rightarrow[p] \times[q]$ from $(0,0)$ to $(p, q)$. It induces a map $\lambda_{*}: \Delta^{p+q} \rightarrow \Delta^{p} \times \Delta^{q}$. A shuffle has a signum $\operatorname{sgn}(\lambda)$ (cf. [tD08, p.240]). Working instead with normalized complexes, the following map is a canonical section of $A W$ :

Definition 5.2. The Eilenberg-Zilber map $E Z$ (or shuffle map) is

$$
\begin{aligned}
& E Z: C(A) \otimes C(B) \rightarrow C(A \otimes B) \\
& E Z(a \otimes b)=\sum_{\lambda \in \Sigma(p, q)} \operatorname{sgn}(\lambda) \cdot(a \otimes b) \circ \lambda_{*},
\end{aligned}
$$


where $a: \Delta^{p} \rightarrow A$ in $C(A)_{p}$ and $b: \Delta^{q} \rightarrow B$ in $C(B)_{q}$.

We will mainly apply these maps for simplicial abelian groups $A=\tilde{\mathbb{Z}} K$, $B=\tilde{\mathbb{Z}} L$ where it should be noted that

$$
\tilde{\mathbb{Z}} K \otimes \tilde{\mathbb{Z}} L=\tilde{\mathbb{Z}}(K \wedge L), \quad K, L \in \operatorname{Set}_{\Delta}^{*} .
$$

Remark 5.3. There are version of $A W, E Z$ for the normalized complex as well, and below it is possible to work with them instead.

\subsubsection{Structure Maps of the Cocycle Spectrum}

Definition 5.4. Fix a $\mathbb{Z}$-graded real vector space $V^{*}$, which we will view as a chain complex, having $n$-th chain group $V_{n}=V^{-n}$ and zero differential. The vector space of reduced $n$-cocycles of $K \in$ Set $_{\Delta}^{*}$ (with coefficients in $V$ ) is the vector space of chain maps

$$
\tilde{Z}^{n}(K)=\mathbf{C h}_{\geq 0}(\tilde{C}(K), V[-n]), \quad n \in \mathbb{Z} .
$$

Here $[k]$ denotes the shift of a chain complex, given by $C[k]_{*}=C_{k+*}$.

We may view $\tilde{Z}^{n}:$ Set $_{\Delta}^{*} \rightarrow$ Vect $_{\mathbb{R}}$ as a functor. In particular, the cosimplicial object $\Delta_{+}^{\bullet}$ of $\mathbf{S e t}_{\Delta}^{*}$ gives rise to simplicial vector spaces (these are always Kan complexes [GJ09, Lemma I.3.4])

$$
\tilde{Z}^{n}\left(K \wedge \Delta_{+}^{\bullet}\right), \quad n \in \mathbb{Z}
$$

We shall place these vector spaces inside a spectrum. That is, we will produce weak equivalences

$$
\tilde{Z}^{n-1}\left(K \wedge \Delta_{+}^{\bullet}\right) \rightarrow \Omega \tilde{Z}^{n}\left(K \wedge \Delta_{+}^{\bullet}\right)
$$

Remark 5.5. The spaces (35) are equivalent to the value of the Dold-Kan functor $D K: \mathbf{C h}_{\geq 0} \rightarrow s \mathbf{A b}$ on the truncated mapping chain complex

$$
\tau_{\geq 0} \operatorname{Hom}(\tilde{C}(K), V[-n]) .
$$

In particular, 35 is equivalent to the mapping $\operatorname{space}^{\operatorname{Map}_{\mathrm{Ch}}}(\tilde{C}(K), V[-n])$ in the $\infty$-category $\mathrm{Ch}$ of all chain complexes. The cocycle spectrum may therefore be viewed as a kind of function spectrum construction. 
We have a diagram of quasi-isomorphisms

$$
\tilde{C}\left(K \wedge \Delta_{+}^{\bullet} \wedge S^{1}\right) \underset{E Z}{\stackrel{A W^{\prime}}{\rightleftarrows}} \tilde{C}\left(K \wedge \Delta_{+}^{\bullet}\right) \otimes \tilde{C}\left(S^{1}\right) \underset{\text { id } \otimes \text { incl }}{\stackrel{\mathrm{id} \otimes \mathrm{pr}}{\rightleftarrows}} \tilde{C}\left(K \wedge \Delta_{+}^{\bullet}\right) \otimes \mathbb{Z}[1] .
$$

The complex on the right may be identified with the shift $\tilde{C}\left(K \wedge \Delta_{+}^{\bullet}\right)[-1]$.

Lemma 5.6. There is a canonical isomorphism of simplicial sets

$$
\Omega \tilde{Z}^{n}\left(K \wedge \Delta_{+}^{\bullet}\right) \cong \tilde{Z}^{n}\left(K \wedge \Delta_{+}^{\bullet} \wedge S^{1}\right)
$$

Proof. The usual subdivision of the prism in terms of the maps $h_{i}: \Delta^{k+1} \rightarrow$ $\Delta^{k} \times \Delta^{1}(i=0, \ldots, k)$ [GJ09, p.17] leads to a coequalizer diagram in Set $_{\Delta}^{*}$

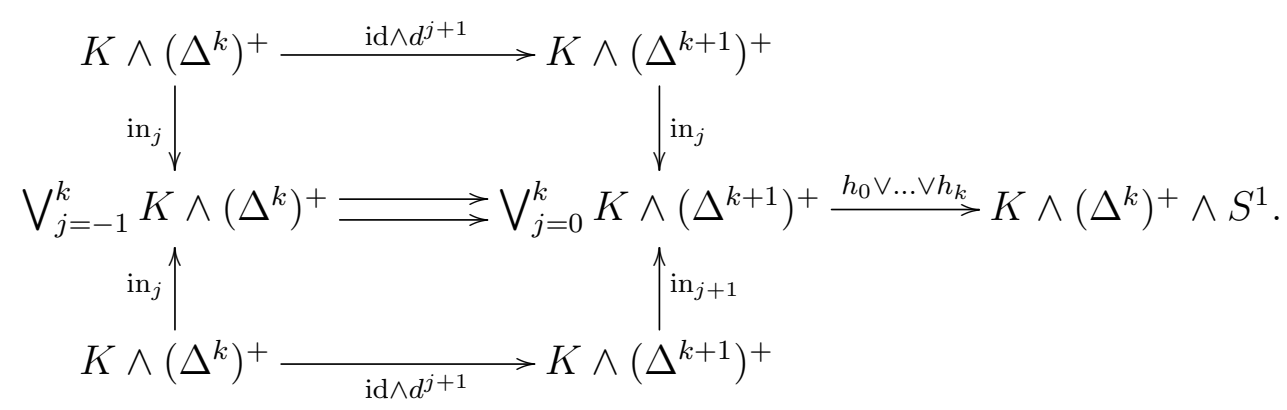

Here, the righthand vertical maps $\operatorname{in}_{l}$ map to the base point if $l=-1, k+1$. In view of the description of the simplicial loop space in Corollary A.18, an element of $\Omega \tilde{Z}^{n}\left(K \wedge\left(\Delta^{k}\right)^{+}\right)$amounts to a map to $V[-n]$ on $\tilde{C}$ of this coequalizer. Since $\tilde{C}$ preserves colimits, we conclude the result.

We remind the reader that $V$ is suppressed from the notation (35).

Lemma 5.7. The homotopy groups $\pi_{i}\left(\tilde{Z}^{n}\left(K \wedge \Delta_{+}^{\bullet}\right), 0\right)$ are given by cohomology $\tilde{H}^{n-i}(K ; V)$ with coefficients in $V$.

A similar statement appears without proof in [HS05, D.13] and also contains a slight inaccuracy (the complex needs to continue to the right). It seems better therefore to prove our assertion.

The homotopy groups of a simplicial group (or vector space, etc.) are defined as the simplicial homotopy groups of the underlying simplicial set 
(110). We will use that the simplicial homotopy groups $\pi_{i}(A, 0)$ of a simplicial abelian group $A$ can be identified with the homology of the corresponding Moore complex [GJ09, Corollary III.2.7].

Proof. The proof has two parts:

(i) For each $n \in \mathbb{Z}$, the simplicial abelian group $\tilde{C}^{n}\left(K \wedge \Delta_{+}^{\bullet} ; V\right)$ of all $n$-cochains with coefficients in $V$ is contractible.

Using the obvious abbreviations (for example, $B^{n}=\tilde{B}^{n}\left(K \wedge \Delta_{+}^{\bullet} ; V\right)$ ) we then show:

(ii) The short exact sequences $0 \rightarrow Z^{n} \rightarrow C^{n} \rightarrow B^{n+1} \rightarrow 0$ and $0 \rightarrow B^{n} \rightarrow$ $Z^{n} \rightarrow H^{n} \rightarrow 0$ of the Moore complexes imply Lemma 5.7.

Here, the Moore complex construction refers to 'the •-direction' and therefore produces a positive chain complex (regardless of $V$ ).

To prove the first assertion, we use 'extension by zero' [HS05, D.11]:

$$
H_{k}: \tilde{C}^{n}\left(K \wedge \Delta_{+}^{k} ; V\right) \rightarrow \tilde{C}^{n}\left(K \wedge \Delta_{+}^{k+1} ; V\right)
$$

sends $u \in \tilde{C}^{n}\left(K \wedge \Delta_{+}^{k} ; V\right)$ to the $V[-n]$-valued cochain on $\tilde{C}\left(K \wedge \Delta_{+}^{k+1}\right)$ which is given by $u$ on simplices contained in $K \wedge \Delta_{+}^{k}$ (viewed as the face opposite to 0 ) and takes every other simplex to zero. The maps $H_{k}$ define a chain homotopy from the identity to zero, so the chain complex belonging to $\tilde{C}^{n}\left(K \wedge \Delta_{+}^{\bullet} ; V\right)$ is acyclic: In detail, $H_{k}: \tilde{C}^{n}\left(K \wedge \Delta_{+}^{k} ; V\right) \rightarrow \tilde{C}^{n}\left(K \wedge \Delta_{+}^{k+1} ; V\right)$ is given by

$$
\left(H_{k} u\right)\left(\Delta^{n} \stackrel{\sigma}{\rightarrow} K \wedge \Delta_{+}^{k+1}\right)= \begin{cases}u(\tau) & \left(\exists \tau: \sigma=\left(\operatorname{id}_{K} \wedge d^{0}\right) \circ \tau\right) \\ 0 & \text { (else) }\end{cases}
$$

Observation: We have $\left(\mathrm{id} \wedge d^{i}\right)^{*}\left(H_{k} u\right)= \begin{cases}u & (i=0), \\ H_{k+1}\left(\left(\mathrm{id} \wedge d^{i-1}\right)^{*} u\right) & (i \neq 0) .\end{cases}$

Proof. Clearly, $\left(\mathrm{id} \wedge d^{0}\right)^{*}\left(H_{k} u\right)=u$. Suppose $i>0$. In case $\left(\mathrm{id} \wedge d^{i}\right) \circ \sigma=(\mathrm{id} \wedge$ $\left.d^{0}\right) \circ \kappa$ for some $\kappa$ we have $\left(\mathrm{id} \wedge d^{i}\right)^{*}\left(H_{k} u\right)(\sigma)=u(\kappa)$ and $\left(\mathrm{id} \wedge d^{i}\right)^{*}\left(H_{k} u\right)(\sigma)=0$ otherwise. But this is also the value of

$$
H_{k+1}\left(\left(\mathrm{id} \wedge d^{i-1}\right)^{*} u\right)(\sigma)= \begin{cases}u\left(\left(\mathrm{id} \wedge d^{i-1}\right) \circ \tau\right) & \left(\exists \tau: \sigma=\left(\mathrm{id} \wedge d^{0}\right) \circ \tau\right), \\ 0 & (\text { else }),\end{cases}
$$

due to the following observation (applied to the second component): 
Suppose $\sigma \in \Delta_{n}^{k}$. If $d^{i} \circ \sigma=d^{0} \circ \kappa$ for some $\kappa$, then there exists $\tau$ with $\sigma=d^{0} \circ \tau$. Moreover, $\kappa=d^{i-1} \circ \tau$.

The observation holds because $\sigma$ corresponds to a map $\sigma:[n] \rightarrow[k-1]$ and the equation $d^{i} \sigma=d^{0} \kappa$ shows that 0 is not in the image of $\sigma$, so one may factor $\sigma=d^{0} \tau$. Since $d^{0}$ is injective, $d^{0} d^{i-1} \tau=d^{i} d^{0} \tau=d^{i} \sigma=d^{0} \kappa$ implies $\kappa=d^{i-1} \circ \tau$.

Using this observation, we calculate that $\left(H_{k}\right)$ defines a chain homotopy

$$
\begin{aligned}
& \sum_{i=0}^{k+1}\left(\mathrm{id} \wedge d^{i}\right)^{*} H_{k} u+\sum_{j=0}^{k}(-1)^{j} H_{k+1}\left[\left(\mathrm{id} \wedge d^{j}\right)^{*} u\right] \\
= & u+\sum_{i=1}^{k+1}\left(\mathrm{id} \wedge d^{i}\right)^{*} H_{k} u+\sum_{i=1}^{k+1}(-1)^{i-1} H_{k+1}\left[\left(\mathrm{id} \wedge d^{i-1}\right)^{*} u\right]=u,
\end{aligned}
$$

where we have reindexed the second summation according to $j=i-1$. Using [GJ09, III.2.7], we conclude (i).

To deduce (ii), we consider long exact sequences in homology. First note that the projection induces an isomorphism from $\tilde{H}^{n}\left(K \wedge \Delta_{+}^{\bullet} ; V\right)$ to $\tilde{H}^{n}(K ; V)$. The differential $\sum(-1)^{i}\left(d^{i}\right)^{*}$ then corresponds to 1 or 0 , depending on the parity of the dimension. The homology of the simplicial abelian group $\tilde{H}^{n}\left(K \wedge \Delta_{+}^{\bullet} ; V\right)$ is therefore $\tilde{H}^{n}(K ; V)$ in degree 0 and zero else. Using (i) and the long exact sequence

$$
\cdots \stackrel{\partial}{\rightarrow} H_{*}\left(Z^{n}\right) \rightarrow H_{*}\left(C^{n}\right) \rightarrow H_{*}\left(B^{n+1}\right) \stackrel{\partial}{\rightarrow} \cdots
$$

we deduce that $H_{0}\left(B^{n+1}\right)=0$ for each $n$. The other long exact sequence

$$
\cdots \stackrel{\partial}{\rightarrow} H_{*}\left(B^{n}\right) \rightarrow H_{*}\left(Z^{n}\right) \rightarrow H_{*}\left(H^{n}\right) \stackrel{\partial}{\rightarrow} \cdots
$$

now implies $\pi_{0}\left(\tilde{Z}^{n}\left(K \wedge \Delta_{+}^{\bullet} ; V\right), 0\right)=H_{0}\left(Z^{n}\right)=H_{0}\left(H^{n}\right)=\tilde{H}^{n}(K ; V)$. Using this and Lemma 5.6 we may now calculate

$$
\begin{aligned}
\pi_{i}\left(\tilde{Z}^{n}\left(K \wedge \Delta_{+}^{\bullet}\right), 0\right) & \cong \pi_{0}\left(\Omega^{i} \tilde{Z}^{n}\left(K \wedge \Delta_{+}^{\bullet}\right), 0\right) \cong \pi_{0}\left(\tilde{Z}^{n}\left(K \wedge \Delta_{+}^{\bullet} \wedge S^{i}\right), 0\right) \\
& =\tilde{H}^{n}\left(K \wedge S^{i} ; V\right) \cong \tilde{H}^{n-i}(K ; V)
\end{aligned}
$$


Remark 5.8. For every pointed space $X$, the simplicial abelian group $\tilde{Z}^{n}(X \wedge$ $\left.\left|\Delta_{+}^{\bullet}\right| ; V\right)$ also has $i$-th homotopy group $\tilde{H}^{n-i}(X ; V)$. We need only to change the use of Lemma 5.6 to the assertion that the canonical map

$$
\tilde{Z}^{n}\left(X \wedge\left|\Delta_{+}^{\bullet}\right| \wedge\left|S^{1}\right| ; V\right) \rightarrow \Omega \tilde{Z}^{n}\left(X \wedge\left|\Delta_{+}^{\bullet}\right| ; V\right)
$$

given by 'subdivision of the prism' is a weak equivalence of simplicial abelian groups [HS05, (4.45)].

Now for the definition of the structure maps $(36)$. We have

$$
\begin{aligned}
& \Omega \tilde{Z}^{n}\left(K \wedge \Delta_{+}^{\bullet}\right) \cong \tilde{Z}^{n}\left(K \wedge \Delta_{+}^{\bullet} \wedge S^{1}\right)=\mathbf{C h}_{\geq 0}\left(\tilde{C}\left(K \wedge \Delta_{+}^{\bullet} \wedge S^{1}\right), V[-n]\right), \\
& \tilde{Z}^{n-1}\left(K \wedge \Delta_{+}^{\bullet}\right)=\mathbf{C h}_{\geq 0}\left(\tilde{C}\left(K \wedge \Delta_{+}^{\bullet}\right) \otimes \mathbb{Z}[1], V[-n]\right) .
\end{aligned}
$$

Definition 5.9. The structure maps $\tilde{Z}^{n-1}\left(K \wedge \Delta_{+}^{\bullet}\right) \rightarrow \Omega \tilde{Z}^{n}\left(K \wedge \Delta_{+}^{\bullet}\right)$ in (36) are defined as pullback $A W^{*}(\mathrm{id} \otimes \mathrm{pr})^{*}$ along (37).

Lemma 5.10. The maps 36 are weak equivalences.

Proof. Since $\tilde{Z}^{n}\left(K \wedge \Delta_{+}^{\bullet} \wedge S^{1}\right)$ is isomorphic to $\Omega \tilde{Z}^{n}\left(K \wedge \Delta_{+}^{\bullet}\right)$, we are reduced to proving that

$$
\tilde{Z}^{n-1}\left(K \wedge \Delta_{+}^{\bullet}\right) \rightarrow \tilde{Z}^{n}\left(K \wedge \Delta_{+}^{\bullet} \wedge S^{1}\right)
$$

is a weak equivalence. But, applying the $i$-th homotopy group, this map induces the suspension isomorphism

$$
\tilde{H}^{n-1-i}(K ; V) \rightarrow \tilde{H}^{n-i}\left(K \wedge S^{1} ; V\right) .
$$

As already mentioned, it will be more convenient to work instead with the following weak inverse of $(36)$ :

$$
(\mathrm{id} \otimes \mathrm{incl})^{*} E Z^{*}: \Omega \tilde{Z}^{n}\left(K \wedge \Delta_{+}^{\bullet}\right) \rightarrow \tilde{Z}^{n-1}\left(K \wedge \Delta_{+}^{\bullet}\right) .
$$

\subsection{Chern Character Transformation}

We view $\tilde{Z}^{n}\left(K \wedge \Delta_{+}^{\bullet}\right)$ as a cospectrum because we wish to define a map

$$
\operatorname{ch}: \operatorname{Hom}(K, E) \rightarrow \tilde{Z}^{n}\left(K \wedge \Delta_{+}^{\bullet}\right)
$$


and in 'elementary' treatments of spectra one usually requires that maps commute strictly with the structure maps $\varsigma^{6}$. We will instead define a cospectrum map (40).

We will work in this chapter with a spectrum $\left(E_{n}, \varepsilon_{n}\right)$ of pointed Kan complexes and suppose that the adjoint structure maps

$$
\varepsilon_{n}^{\text {adj }}: E_{n} \rightarrow \Omega E_{n+1}
$$

are isomorphisms of simplicial sets. Beginning with a spectrum as in (15), such a spectrum may be obtained by applying the singular space functor:

$$
\operatorname{sing} E_{n} \cong \operatorname{sing}\left(\Omega E_{n+1}\right) \cong \Omega \operatorname{sing}\left(E_{n+1}\right)
$$

To explain the isomorphism, note that a $k$-simplex of $\operatorname{sing}\left(\Omega E_{n+1}\right)$ amounts to a simplicial map $S^{1} \wedge\left|\Delta_{+}^{k}\right| \rightarrow E_{n+1}$, while a $k$-simplex of $\Omega \operatorname{sing}\left(E_{n+1}\right)$ amounts to a map $S^{1} \wedge \Delta_{+}^{k} \rightarrow \operatorname{sing}\left(E_{n+1}\right)\left(\right.$ or $\left.\left|S^{1} \wedge \Delta_{+}^{k}\right| \rightarrow E_{n+1}\right)$. The isomorphism now follows from the fact that $|\cdot|$ preserves products GJ09, Proposition I.2.4], so that $\left|S^{1} \wedge \Delta_{+}^{k}\right| \cong\left|S^{1}\right| \wedge\left|\Delta_{+}^{k}\right|$. By definition of singular cochains (and of $\int_{S^{1}}$ ), our fundamental cocycles 16 ) then amount to elements

$$
\iota_{n} \in \tilde{Z}^{n}\left(E_{n} ; V\right), \quad n \in \mathbb{Z},
$$

with the property that each of the following diagrams commute:

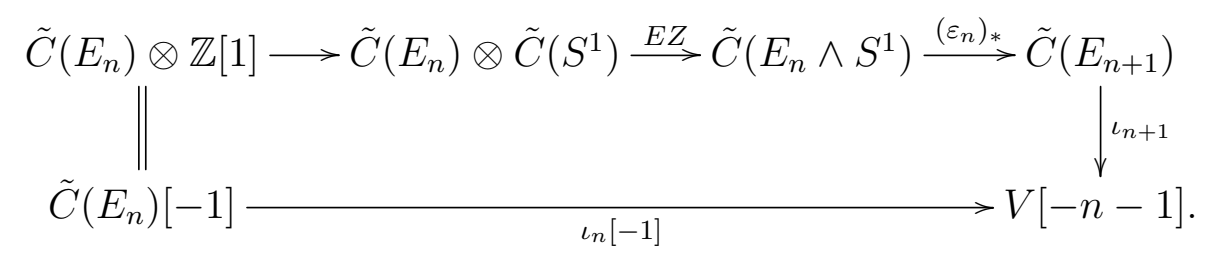

\subsubsection{Mapping and Loop Spaces}

We recall in this section the most commonly used notions of mapping spaces. The mapping space $\operatorname{Hom}(X, Y)$ [GJ09, Section I.5] between simplicial sets $X, Y$ has as $n$-simplices all simplicial maps

$$
X \times \Delta^{n} \rightarrow Y
$$

\footnotetext{
${ }^{6}$ It is possible to develop stable homotopy with maps which commute with the structure maps up to homotopy, provided one keeps track of them (and uses them to define the notion of homotopy of such maps). This has already been observed in [Ada95, p.141] and is the homotopy category hSp of the $\infty$-category of spectra in [Lur07c].
} 
We have an adjunction $\operatorname{Set}_{\Delta}(K, \operatorname{Hom}(X, Y)) \cong \operatorname{Hom}(X \times K, Y)$ given by evaluation [GJ09, Proposition I.5.1]. These definitions turn the category of simplicial sets into a simplicial category. Another common example is the category of pointed simplicial sets Set $_{\Delta}^{*}$, which is tensored over simplicial sets via [GJ09, p.84]

$$
X \otimes K=X \wedge K_{+}, \quad X \in \mathbf{S e t}_{\Delta}^{*}, K \in \mathbf{S e t}_{\Delta} .
$$

The mapping space is given by $\operatorname{Hom}_{*}(X, Y)_{n}=\operatorname{Set}_{\Delta}^{*}\left(X \otimes \Delta^{n}, Y\right)$. For example, the loop space is defined by

$$
\Omega(X)=\operatorname{Hom}_{*}\left(S^{1}, X\right) .
$$

The suspension is defined by $\Sigma X=X \wedge S^{1}$. We have isomorphisms

$$
\operatorname{Hom}_{*}(\Sigma X, Y) \cong \operatorname{Hom}_{*}(X, \Omega Y)
$$

Recall that a simplicial category (in the sense of [GJ09, Definition II.2.1]) is a category $\mathscr{C}$ along with functors

$$
\begin{aligned}
\operatorname{Hom}_{\mathscr{C}}: \mathscr{C}^{\mathrm{op}} \times \mathscr{C} & \rightarrow \operatorname{Set}_{\Delta}, \\
\otimes: \mathscr{C} \times \operatorname{Set}_{\Delta} & \rightarrow \mathscr{C} \\
\operatorname{hom}_{\mathscr{C}}: \operatorname{Set}_{\Delta}^{\mathrm{op}} \times \mathscr{C} & \rightarrow \mathscr{C} .
\end{aligned}
$$

such that

1. The zero simplices of $\operatorname{Hom}_{\mathscr{C}}(X, Y)$ is given by the set $\mathscr{C}(X, Y)$ of morphisms in the category $\mathscr{C}$.

2. We have adjunctions [GJ09, p.83]

$$
\begin{aligned}
\operatorname{Hom}_{\operatorname{Set}_{\Delta}}\left(K, \operatorname{Hom}_{\mathscr{C}}(X, Y)\right) & \cong \operatorname{Hom}_{\mathscr{C}}(X \otimes K, Y) \\
& \cong \operatorname{Hom}_{\mathscr{C}}(X, \operatorname{hom}(K, Y)) .
\end{aligned}
$$

3. $X \otimes(K \times L) \cong(X \otimes K) \otimes L$.

Parts of this data is determined by the other parts, for example, we have $\operatorname{Hom}_{\mathscr{C}}(X, Y)_{n} \cong \mathscr{C}\left(X \otimes \Delta^{n}, Y\right)$.

The most obvious example is the category of simplicial sets with $\otimes=\times$ and $\operatorname{Hom}_{\mathbf{S e t}_{\Delta}}(X, Y)=\operatorname{hom}(X, Y)$ (whose $n$-simplices are simplicial maps 
$X \times \Delta^{n} \rightarrow Y$ ). If $\mathscr{C}=\operatorname{Set}_{\Delta}^{*}$ is the simplicial category of pointed simplicial sets [GJ09, p.84], we have

$$
X \otimes K=X \wedge K_{+}, \quad \operatorname{hom}(K, X)=\operatorname{Hom}_{\text {Set }_{\Delta}}(K, X) .
$$

Here, $\operatorname{hom}(K, X)$ has the constant map given by the base-point of $X$ as base-point. The $n$-simplices of the mapping space are simplicial maps

$$
X \wedge \Delta_{+}^{n} \rightarrow Y
$$

If understood from the context that $X, Y$ are to be viewed as pointed simplicial sets, we will also write $\operatorname{Hom}(X, Y)$ for $\operatorname{Hom}_{\mathbf{S e t}_{\Delta}^{*}}(X, Y)$. Using the constant base-point map allows us to view $\mathbf{H o m}_{\text {Set }_{\Delta}^{*}}(X, Y)$ as a pointed simplicial set.

The adjunction above specializes to the isomorphisms of simplicial sets

$$
\operatorname{Hom}(K, \Omega Y) \cong \operatorname{Hom}_{\mathbf{S e t}_{\Delta}^{*}}\left(S_{+}^{1} \wedge K, Y\right) \cong \Omega \operatorname{Hom}(K, Y) .
$$

Note that $S_{+}^{1} \wedge K$ is the unreduced suspension of $K$. If we base-point $\operatorname{Hom}_{\mathbf{S e t}_{\Delta}^{*}}(X, Y)$ by the constant base-point map, we have an adjunction in Set $_{\Delta}^{*}$

$$
(-\wedge X) \dashv \operatorname{Hom}_{\text {Set }_{\Delta}^{*}}(X,-)
$$

Proof. We must show that for any pointed simplicial set $A$ we have $\operatorname{Set}_{\Delta}^{*}(A \wedge$ $X, Y) \cong \operatorname{Set}_{\Delta}^{*}\left(A, \mathbf{H o m}_{\mathbf{S e t}_{\Delta}^{*}}(X, Y)\right)$. An unpointed simplicial map $f: A \rightarrow$ $\operatorname{Hom}_{\text {Set }_{\Delta}^{*}}(X, Y)$ amounts by the adjunction 2. above to a pointed map $g$ : $A_{+} \wedge X \rightarrow Y$, that is, a map $A \times X \rightarrow Y$ which takes $A \times\{$ pt $\}$ to the base-point. The condition that $f$ be pointed means precisely that $g$ take $\{\mathrm{pt}\} \times X$ to the base-point as well, that is, defines a map on the smash product $A \wedge X$.

From this adjunction we deduce pointed isomorphisms

$$
\operatorname{Hom}_{\text {Set }_{\Delta}^{*}}(X, \Omega Y) \cong \operatorname{Hom}_{\text {Set }}^{*}(\Sigma X, Y) \cong \Omega \operatorname{Hom}_{\text {Set }}^{*}(X, Y) .
$$

Indeed, simplices in these simplicial sets are described respectively by

$$
\begin{gathered}
X \wedge \Delta_{+}^{\bullet} \rightarrow \Omega Y, \\
X \wedge S^{1} \wedge \Delta_{+}^{\bullet} \rightarrow Y, \\
S^{1} \wedge \Delta_{+}^{\bullet} \wedge X \rightarrow Y .
\end{gathered}
$$


The category CGHaus of compactly generated Hausdorff spaces is also a simplicial category, but we will instead usually use the internal hom

$$
Y^{X}=\{X \rightarrow Y \text { continuous }\}
$$

endowed with the compact-open topology. We then have DK01, Theorem 6.5] homeomorphisms

$$
Z^{X \times Y} \cong\left(Z^{Y}\right)^{X}
$$

If $X, Y \in$ CGHaus* $^{*}$ are pointed, we let

$$
Y^{X}=\{X \rightarrow Y \text { continuous, pointed }\}
$$

topologized as a subspace of the unpointed exponential. The above homeomorphism then restricts to a pointed homeomorphism

$$
Z^{X \wedge Y} \cong\left(Z^{Y}\right)^{X}
$$

For pointed $X$, we let $\Omega X=X^{S^{1}}$ and $\Sigma X=X \wedge S^{1}$. Then

$$
(\Omega Y)^{X}=\left(Y^{S^{1}}\right)^{X} \cong Y^{\Sigma X} \cong\left(Y^{X}\right)^{S^{1}}=\Omega\left(Y^{X}\right)
$$

\subsubsection{Mapping Spectra}

Let $\left(F_{n}\right)_{n \in \mathbb{Z}}$ be a spectrum of pointed Kan complexes so that we have pointed weak equivalences

$$
F_{n} \stackrel{\simeq}{\longrightarrow} \Omega F_{n+1} \text {. }
$$

Both sides are fibrant. By applying (the dual of) [GJ09, II.3.15] to Set$_{\Delta}^{*}$ we deduce that for any (automatically cofibrant) $K \in \mathbf{S e t}_{\Delta}^{*}$ the structure maps induce weak equivalences

$$
\operatorname{Hom}\left(K, F_{n}\right) \stackrel{\simeq}{\longrightarrow} \operatorname{Hom}\left(K, \Omega F_{n+1}\right) \cong \Omega \operatorname{Hom}\left(K, F_{n+1}\right) .
$$

The last isomorphism is 42 .

Definition 5.11. For a pointed simplicial set $K$, the $\operatorname{spaces} \operatorname{Hom}\left(K, F_{n}\right)$ along with the structure maps 43 form the mapping spectrum $\operatorname{Hom}(K, F)$. 


\subsubsection{Construction of $\mathrm{ch}$}

In this subsection, we will define the cospectrum map from above

$$
\operatorname{ch}: \operatorname{Hom}(K, E) \rightarrow \tilde{Z}^{n}\left(K \wedge \Delta_{+}^{\bullet}\right) \text {. }
$$

Definition 5.12. The $n$-th level of the map 40 is defined as 'pullback along the fundamental cocycles:'

$$
\begin{aligned}
\operatorname{ch}: \operatorname{Hom}\left(K, E_{n}\right) & \longrightarrow \tilde{Z}^{n}\left(K \wedge \Delta_{+}^{\bullet}\right) \\
\left(f: K \wedge \Delta_{+}^{\bullet} \rightarrow E_{n}\right) & \longmapsto\left(\tilde{C}\left(K \wedge \Delta_{+}^{\bullet}\right) \stackrel{f_{*}}{\longrightarrow} \tilde{C}\left(E_{n}\right) \stackrel{\iota_{n}}{\longrightarrow} V[-n]\right)=f^{*} \iota_{n} .
\end{aligned}
$$

Proposition 5.13. The maps in Definition 5.12 commute strictly with the cospectrum structure maps (the inverse of (43) and the canonical section (39) ).

Proof. For each $f: K \wedge \Delta_{+}^{\bullet} \wedge S^{1} \rightarrow E_{n+1}$ let $g: K \wedge \Delta_{+}^{\bullet} \rightarrow E_{n}$ be the unique map with $\varepsilon_{n} \circ\left(g \wedge 1_{S^{1}}\right)=f$ (given by $\left.K \wedge \Delta_{+}^{\bullet} \stackrel{f^{\text {adj }}}{\longrightarrow} \Omega E_{n+1} \stackrel{\left(\varepsilon_{n}^{\text {adj }}\right)^{-1}}{\longrightarrow} E_{n}\right)$. Unwinding the definitions, we see that we need to compare

$$
\begin{gathered}
\tilde{C}\left(K \wedge \Delta_{+}^{\bullet}\right) \otimes \mathbb{Z}[1] \stackrel{1 \otimes \text { incl }}{\longrightarrow} \tilde{C}\left(K \wedge \Delta_{+}^{\bullet}\right) \otimes \tilde{C}\left(S^{1}\right) \stackrel{E Z}{\longrightarrow} \tilde{C}\left(K \wedge \Delta_{+}^{\bullet} \wedge S^{1}\right) \\
\stackrel{f_{*}}{\longrightarrow} \tilde{C}\left(E_{n+1}\right) \stackrel{\iota_{n+1}}{\longrightarrow} V[-n-1]
\end{gathered}
$$

with the shift of

$$
\tilde{C}\left(K \wedge \Delta_{+}^{\bullet}\right) \stackrel{g_{*}}{\longrightarrow} \tilde{C}\left(E_{n}\right) \stackrel{\iota_{n}}{\longrightarrow} V[-n] .
$$

By naturality of $E Z$ and the compatibility 41) we have a commutative diagram in which the maps we wish to compare appear:

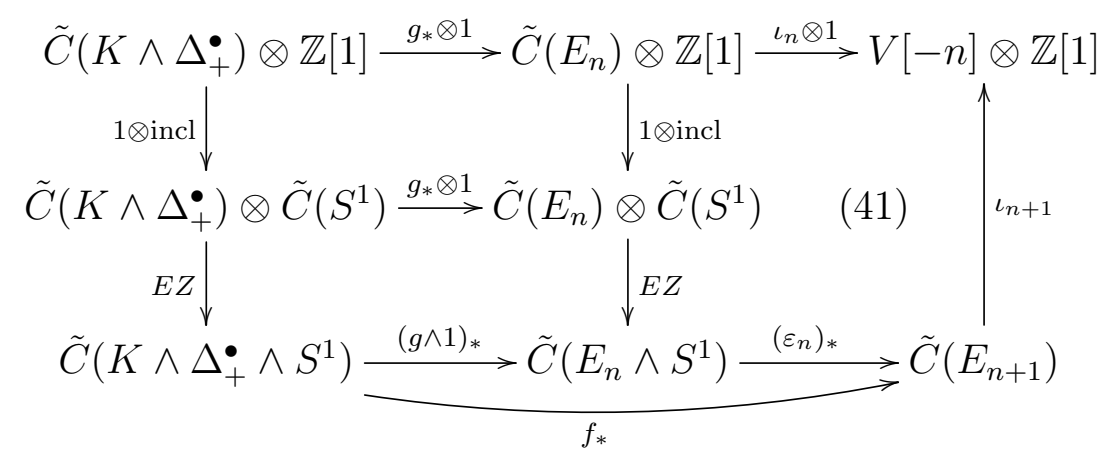




\subsection{The Additivity Coherence Conditions}

\subsubsection{Chern Character as a Functor}

The Chern character (40) may be regarded as a functor

$$
\mathrm{Kan}^{*} \rightarrow \operatorname{Ar}(\mathbf{c o S p t})
$$

into the category of arrows in coSpt, whose morphisms are commutative squares. Post-composing with the fundamental groupoid construction $\left(\Pi_{1}\right)_{-n}$ of cospectra takes us to the category $\operatorname{Ar}(\mathbf{2 B r M o n C a t})$ :

Theorem 5.14. For each $K \in \mathrm{Kan}^{*}$, the Chern character induces a natural family of morphisms in 2BrMonCat

$$
\left(\Pi_{1}\right)_{-n} \operatorname{Hom}(K, E) \longrightarrow\left(\Pi_{1}\right)_{-n} \tilde{Z}\left(K \wedge \Delta_{+}^{\bullet}\right) .
$$

Let us unwind what this means in more detail. First we recall the definition of $\left(\Pi_{1}\right)_{-n} \operatorname{Hom}(K, E)$ : it denotes category $\Pi_{1} \operatorname{Hom}\left(K, E_{n}\right)$ and is a braided monoidal category in two ways ' $($ '), ' $\ominus$ ' (arising essentially from $\left.E_{n} \cong \Omega^{2} E_{n+2}\right)$. We had also defined a braided monoidal structure on the identity functor (stemming from an Eckmann-Hilton argument)

$$
e^{\oplus, \ominus}:\left(\Pi_{1} \operatorname{Hom}\left(K, E_{n}\right), \oplus\right) \rightarrow\left(\Pi_{1} \operatorname{Hom}\left(K, E_{n}\right), \ominus\right) .
$$

The structure on the category $\left(\Pi_{1}\right)_{-n} \tilde{Z}\left(K \wedge \Delta_{+}^{\bullet}\right)$ is defined in the same way. It was seen above that we may identify it (in 2BrMonCat) with $\left(\Pi_{1}^{a b}\right)_{-n} \tilde{Z}\left(K \wedge \Delta_{+}^{\bullet}\right)$, which as we recall denotes the category $\Pi_{1} \tilde{Z}^{n}\left(K \wedge \Delta_{+}^{\bullet}\right)$ with both monoidal structures given by addition ' + ' in the simplicial abelian group and the strict monoidal structure on the identity functor.

The theorem now asserts that the functor $\Pi_{1}$ ch $: \Pi_{1} \operatorname{Hom}\left(K, E_{n}\right) \rightarrow$ $\Pi_{1} \tilde{Z}^{n}\left(K \wedge \Delta_{+}^{\bullet}\right)$ may be viewed as a monoidal functor for each of the structures $\mathrm{ch}^{\oplus}: \oplus \rightarrow+, \operatorname{ch}^{\ominus}: \ominus \rightarrow+$. Since $\Pi_{1}$ ch is a morphism in 2BrMonCat, these two monoidal structures on $\Pi_{1}$ ch are related by the following commutative diagram in braided monoidal categories:

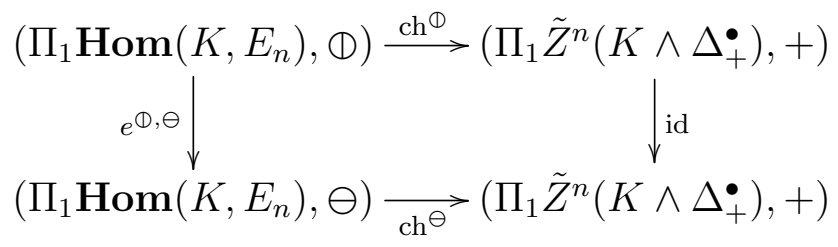




\subsubsection{Transition to Spaces}

We now indicate how Theorem 5.14 specializes to pointed spaces. Suppose still that $\left(E_{n}, \varepsilon_{n}\right)$ is a spectrum whose adjoint structure maps are pointed homeomorphisms and that $X$ is a pointed space. We may then apply our result to $K=\operatorname{sing}(X)$ and $\left(\operatorname{sing}\left(E_{n}\right), \operatorname{sing}\left(\varepsilon_{n}\right)\right)$.

As outlined in Appendix A.3, a space $X$ gives rise to a Kan complex $\operatorname{sing}(X)$ whose $n$-simplices are continuous maps $\left|\Delta^{n}\right| \rightarrow X$. There is a canonical map

$$
\operatorname{sing}\left(Y^{X}\right) \rightarrow \operatorname{Hom}_{\text {Set }_{\Delta}}(\operatorname{sing} X, \operatorname{sing} Y),
$$

which takes an $n$-simplex $\sigma: \Delta^{n} \rightarrow Y^{X}$ to

$$
\Delta^{n} \times \operatorname{sing}(X) \rightarrow \operatorname{sing}\left(\Delta^{n}\right) \times \operatorname{sing}(X)=\operatorname{sing}\left(\Delta^{n} \times X\right) \stackrel{\operatorname{sing}\left(\sigma^{\text {adj }}\right)}{\longrightarrow} \operatorname{sing}(Y) .
$$

The simplicial map (44) is a weak equivalence: applying [GJ09, Lemma II.3.15] to $X \simeq|\operatorname{sing}(X)|$ and $Y$ yields a weak equivalence

$$
\operatorname{sing}\left(Y^{X}\right)=\operatorname{Hom}_{\mathbf{C G}}(X, Y) \simeq \operatorname{Hom}_{\mathbf{C G}}(|\operatorname{sing}(X)|, Y) .
$$

The map (44) is the composition of this equivalence with the equivalence

$$
\operatorname{Hom}_{\mathbf{C G}}(|\operatorname{sing}(X)|, Y) \simeq \operatorname{Hom}_{\mathbf{S e t}_{\boldsymbol{\Delta}}}(\operatorname{sing}(X), \operatorname{sing}(Y))
$$

from [GJ09, p.95].

Definition 5.15. For $X, Y \in \mathbf{C G H a u s}_{*}$, let $\operatorname{Map}(X, Y)=\Pi_{1} Y^{X}=\Pi_{1} \operatorname{sing}\left(Y^{X}\right)$.

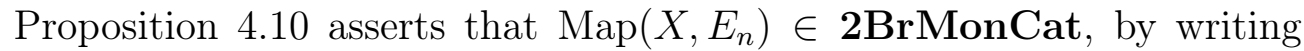
$E_{n} \approx \Omega^{2} E_{n+2}$ and $\operatorname{Map}\left(X, E_{n}\right) \cong \Pi_{1}\left(\Omega^{2} E_{n+2}\right)^{X} \cong \Pi_{1} \Omega^{2}\left(E_{n+2}^{X}\right)$.

Lemma 5.16. The natural equivalence induced by 44

$$
\operatorname{Map}\left(X, E_{n}\right) \rightarrow\left(\Pi_{1}\right)_{-n} \operatorname{Hom}(\operatorname{sing} X, \operatorname{sing} E)
$$

may canonically be viewed as a morphism in 2BrMonCat.

Proof. By definition of $\left(\Pi_{1}\right)_{-n} \operatorname{Hom}(\operatorname{sing} X, \operatorname{sing} E)$ we have morphisms in 2BrMonCat

$$
\begin{aligned}
\left(\Pi_{1}\right)_{-n} \operatorname{Hom}(\operatorname{sing} X, \operatorname{sing} E) & \stackrel{\simeq}{\longleftarrow} \Pi_{1} \Omega^{2} \operatorname{Hom}\left(\operatorname{sing} X, \operatorname{sing} E_{n+2}\right), \\
\Pi_{1} \Omega^{2} \operatorname{Hom}\left(\operatorname{sing} X, \operatorname{sing} E_{n+2}\right) & \stackrel{\simeq}{\rightarrow} \Pi_{1} \Omega^{2}\left|\operatorname{Hom}\left(\operatorname{sing} X, \operatorname{sing} E_{n+2}\right)\right| .
\end{aligned}
$$


As an ordinary category, $\Pi_{1} \Omega^{2} \mathbf{H o m}\left(\operatorname{sing} X, \operatorname{sing} E_{n+2}\right)$ is isomorphic to $\Pi_{1} \Omega^{2} E_{n+2}^{|\operatorname{sing} X|}$ (an object $S^{2} \rightarrow \mathbf{H o m}\left(\operatorname{sing} X, \operatorname{sing} E_{n+2}\right)$ amounts to $S^{2} \wedge \operatorname{sing} X \rightarrow \operatorname{sing}\left(E_{n+2}\right)$ or to $\left.\left|S^{2}\right| \wedge|\operatorname{sing} X| \rightarrow E_{n+2}\right)$. Using Proposition 4.10 , we may view $\Pi_{1} \Omega^{2} E_{n+2}^{|\operatorname{sing} X|}$ as an object of $\mathbf{2 B r M o n C a t}$. The map

$$
E_{n+2}^{|\operatorname{sing} X|} \rightarrow\left|\operatorname{Hom}\left(\operatorname{sing} X, \operatorname{sing} E_{n+2}\right)\right|
$$

taking $f:|\operatorname{sing} X| \rightarrow E_{n+2}$ to its adjoint $\operatorname{sing} X \rightarrow \operatorname{sing} E_{n+2}$ (viewed as a zero simplex of the right hand side), induces a strict morphism (second part of Proposition 4.10

$$
\Pi_{1} \Omega^{2} E_{n+2}^{|\operatorname{sing} X|} \rightarrow \Pi_{1} \Omega^{2}\left|\mathbf{H o m}\left(\operatorname{sing} X, \operatorname{sing} E_{n+2}\right)\right| .
$$

It lifts uniquely via Propositions A.11. A.14 along (B) to a morphism in 2BrMonCat

$$
\Pi_{1} \Omega^{2} E_{n+2}^{|\operatorname{sing} X|} \rightarrow \Pi_{1} \Omega^{2} \operatorname{Hom}\left(\operatorname{sing} X, \operatorname{sing} E_{n+2}\right) .
$$

Pullback along $|\operatorname{sing} X| \rightarrow X$, the map $E_{n+2}^{X} \rightarrow E_{n+2}^{|\operatorname{sing} X|}$, induces another strict morphism (second part of Proposition 4.10)

$$
\operatorname{Map}\left(X, E_{n}\right) \cong \Pi_{1} \Omega^{2} E_{n+2}^{X} \rightarrow \Pi_{1} \Omega^{2} E_{n+2}^{|\operatorname{sing} X|} .
$$

Each of (A), (C), (D) are morphisms in 2BrMonCat, so that their composition 45 is also a morphism in 2BrMonCat.

Definition 5.17. For $X \in$ CGHaus $_{*}$ and a graded vector space $V$, let $\tilde{\mathscr{C}}^{n}(X)$ denote the strict symmetric monoidal groupoid from Example A.3 induced by the homomorphism $\delta: \tilde{C}^{n-1}(X ; V)_{\delta} \rightarrow \tilde{C}^{n}(X ; V)$.

These may be organized into a functor $\tilde{\mathscr{C}}^{n}: \mathbf{C G H a u s}_{*}^{\text {op }} \rightarrow$ SymMonCat $_{\text {strict }}$ by pullback of cochains along maps.

Lemma 5.18. We have strict symmetric monoidal functors, natural in $X$,

$$
\left(\Pi_{1}\right)_{-n} \tilde{Z}\left(\operatorname{sing}(X) \wedge \Delta_{+}^{\bullet}\right)=\Pi_{1} \tilde{Z}^{n}\left(\operatorname{sing}(X) \wedge \Delta_{+}^{\bullet}\right) \rightarrow \tilde{\mathscr{C}}^{n}(X)
$$

given by inclusion on objects (the first equality is by definition). To the class of $f: \tilde{C}\left(\operatorname{sing}(X) \wedge \Delta_{+}^{1}\right) \rightarrow V[-n]$ we assign by (59) the class of the $(n-1)$ cochain $\int_{\Delta^{1}} f$. 
Proof. (46) is well-defined: making use of the abelian group structure, it suffices to show the following: if $u \in \tilde{Z}^{n}\left(\operatorname{sing}(X) \wedge \Delta_{+}^{2}\right)$ with $d_{0} u=d_{2} u=0$, then the $(n-1)$-cochain associated to $d_{1} u$ bounds. This is immediate from Lemma 6.2 (ii). Since $\int_{S^{1}}$ is linear, 46) is a strict monoidal functor. Also $\int_{S^{1}}$ is compatible with pullback, by Lemma 6.2 (i).

Pre- and post-composing the result of Theorem 5.14 with these functors we deduce:

Theorem 5.19. The Chern character induces morphisms in $2 \mathrm{BrMonCat}$

$$
\operatorname{Map}\left(X, E_{n}\right) \stackrel{\mathrm{ch}}{\longrightarrow} \tilde{\mathscr{C}}^{n}(X) .
$$

Explicitly, $\operatorname{ch}(c)=c^{*} \iota_{n}$ on objects and $\operatorname{ch}(h)=\int_{I} h^{*} \iota_{n}$ on morphisms. These are natural in $X$ in the sense that any pointed map $f: X \rightarrow Y$ induces a commutative diagram in $2 \mathrm{BrMonCat:}$

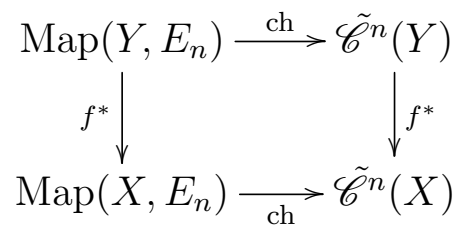

\subsubsection{Display of Coherence Conditions}

Theorem 5.19 is really a result about our fundamental cocycles $\iota_{n}$ and the possibility of coherently chosing cochains $A_{n}$ as in (20). We will make this explicit in this section.

Remark 5.20. For the results of this section it would have sufficed to work in BrMonCat instead of $\mathbf{2 B r M o n C a t}$. This is only needed later for the integration maps.

Fix the associativity and unit constraints $a, r, l$ and the braid $s$ in $\operatorname{Map}\left(X, E_{n}\right)$ :

$$
\begin{aligned}
a: E_{n}^{\times 3} \times I \rightarrow E_{n}, & & \alpha_{n} \circ\left(\alpha_{n} \times \mathrm{id}\right) \simeq \alpha_{n} \circ\left(\mathrm{id} \times \alpha_{n}\right), \\
s: E_{n}^{\times 2} \times I \rightarrow E_{n}, & & \alpha_{n} \circ \text { twist } \simeq \alpha_{n}, \\
r: E_{n} \times I \rightarrow E_{n}, & & \alpha_{n} \circ(\mathrm{id}, \text { const }) \simeq \mathrm{id} .
\end{aligned}
$$

We will write 'E' for 'up to coboundary.' 
Corollary 5.21. Let $\alpha_{n}: E_{n} \times E_{n} \rightarrow E_{n}$ be concatenation of loops (33). There exist cochains $A_{n} \in \tilde{C}^{n-1}\left(E_{n} \times E_{n} ; V\right)$ with

$$
\delta A_{n}=\operatorname{pr}_{1}^{*} \iota_{n}+\operatorname{pr}_{2}^{*} \iota_{n}-\alpha_{n}^{*} \iota_{n} .
$$

These are coherent in the sense that

$$
\begin{aligned}
\operatorname{pr}_{12}^{*} A_{n}+\left(\alpha_{n} \times 1\right)^{*} A_{n} & \equiv \operatorname{pr}_{23}^{*} A_{n}+\left(1 \times \alpha_{n}\right)^{*} A_{n}+\operatorname{ch}(a) & & \text { associative } \\
\text { twist }^{*} A_{n} & \equiv A_{n}+\operatorname{ch}(s) & & \text { commutative } \\
\left(\operatorname{id}_{E_{n}}, \text { const }\right)^{*} A_{n} & \equiv \operatorname{ch}(r) & & \text { unit }
\end{aligned}
$$

Proof. According to Theorem 5.19 (forgetting the second monoidal structure $\ominus$ ) we have natural (in $X$ ) braided monoidal functors

$$
\operatorname{ch}:\left(\operatorname{Map}\left(X, E_{n}\right), \Phi\right) \rightarrow\left(\tilde{\mathscr{C}}^{n}(X),+\right) .
$$

The data of a monoidal functor includes isomorphisms in $\tilde{\mathscr{C}}^{n}(X)$ relating the two tensor products, that is, elements $\operatorname{ch}_{c, d}^{\Phi} \in \tilde{C}^{n-1}(X)_{\delta}$ with

$$
\delta \operatorname{ch}_{c, d}^{\oplus}=\operatorname{ch}(f \oplus g)-\operatorname{ch}(f)-\operatorname{ch}(g) .
$$

For the units we have $\mathrm{ch}_{\text {const }}^{\Phi}=0$, which induces an isomorphism $\delta 0=$ ch(const) -0 . Naturality asserts that we have a commutative diagram of braided monoidal categories

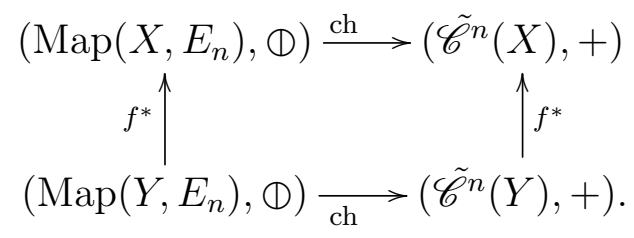

By definition of the composition in $\tilde{\mathscr{C}}^{n}(X)$ and since the vertical functors are strict this means

$$
f^{*} \operatorname{ch}_{c, d}^{\Phi}=\operatorname{ch}_{f^{*} c, f^{*} d}^{\Phi} \quad \text { in } \quad \tilde{C}^{n-1}(X)_{\delta}, \quad c, d: Y \rightarrow E_{n} .
$$

The structure on a braided monoidal functors has to satisfy several coherence conditions:

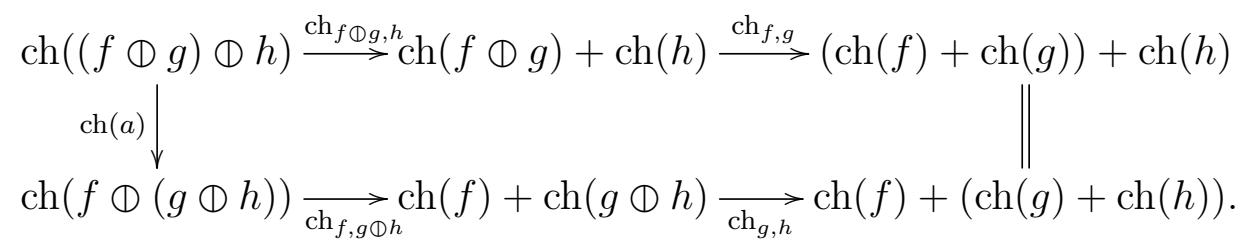




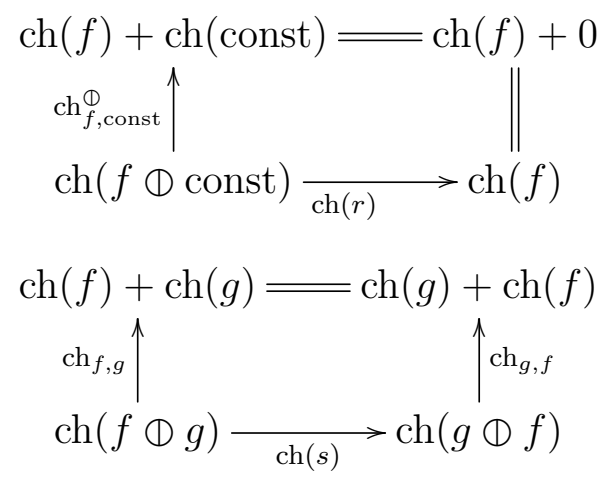

Set $c=\operatorname{pr}_{1}, d=\operatorname{pr}_{2}: E_{n} \times E_{n} \rightarrow E_{n}$ in 48 to define

$$
A_{n}=\operatorname{ch}_{\mathrm{pr}_{1}, \mathrm{pr}_{2}}^{\oplus} .
$$

The three coherence diagrams then express the three equations we wish to prove: the associativity diagram says

$$
\operatorname{ch}_{g, h}^{\Phi}+\operatorname{ch}_{f, g \Phi h}^{\Phi}+\operatorname{ch}(a)=\operatorname{ch}_{f, g}^{\Phi}+\operatorname{ch}_{f \Phi g, h}^{\Phi} \quad \text { in } \quad \tilde{C}^{n-1}(X)_{\delta} .
$$

If we set $f=\mathrm{pr}_{1}, g=\mathrm{pr}_{2}, h=\mathrm{pr}_{3}: E_{n} \times E_{n} \times E_{n} \rightarrow E_{n}$, naturality 49 yields the following implications:

$$
\begin{aligned}
& f=\left(1 \times \alpha_{n}\right)^{*} \operatorname{pr}_{1}, g \oplus h=\left(1 \times \alpha_{n}\right)^{*} \operatorname{pr}_{2} \Rightarrow \mathrm{ch}_{f, g \Phi h}^{\Phi}=\left(1 \times \alpha_{n}\right)^{*} \mathrm{ch}_{\mathrm{pr}_{1}, \mathrm{pr}_{2}}^{\Phi}, \\
& g=\mathrm{pr}_{23}^{*} \mathrm{pr}_{1}, h=\mathrm{pr}_{23}^{*} \mathrm{pr}_{2} \Rightarrow \mathrm{ch}_{g, h}^{\Phi}=\operatorname{pr}_{23}^{*} \mathrm{ch}_{\mathrm{pr}_{1}, \mathrm{pr}_{2}}^{\Phi}, \\
& f\left(\mathbb{D} g=\left(\alpha_{n} \times 1\right)^{*} \operatorname{pr}_{1}, h=(\alpha \times 1)^{*} \operatorname{pr}_{2} \Rightarrow \operatorname{ch}_{f \Phi g, h}^{\Phi}=\left(\alpha_{n} \times 1\right)^{*} \mathrm{ch}_{\mathrm{pr}_{1}, \mathrm{pr}_{2}}^{\Phi}\right. \text {, } \\
& f=\mathrm{pr}_{12}^{*} \mathrm{pr}_{1}, g=\mathrm{pr}_{12}^{*} \mathrm{pr}_{2} \Rightarrow \mathrm{ch}_{f, g}^{\oplus}=\operatorname{pr}_{12}^{*} \mathrm{hh}_{\mathrm{pr}_{1}, \mathrm{pr}_{2}}^{\Phi} \text {. }
\end{aligned}
$$

Inserting these equalities and (50) into (51) then gives

$$
\operatorname{pr}_{23}^{*} A_{n}+\left(1 \times \alpha_{n}\right)^{*} A_{n}+\operatorname{ch}(a)=\operatorname{pr}_{12}^{*} A_{n}+\left(\alpha_{n} \times 1\right)^{*} A_{n} .
$$

Similarly, the second coherence diagram for $f=\operatorname{pr}_{1}$ asserts $\operatorname{ch}(r) \equiv$ $\mathrm{ch}_{\mathrm{pr}_{1}, \mathrm{const}}^{\Phi} \equiv$ (id, const) ${ }^{*} \mathrm{ch}_{\mathrm{pr}_{1}, \mathrm{pr}_{2}}=\left(\mathrm{id}\right.$, const) ${ }^{*} A_{n}$ (the second equivalence is by naturality (49)). The third diagram for $f=\mathrm{pr}_{1}, g=\mathrm{pr}_{2}$ says, using naturality 49 for $\mathrm{pr}_{2}=$ twist ${ }^{*} \mathrm{pr}_{1}, \mathrm{pr}_{1}=$ twist ${ }^{*} \mathrm{pr}_{2}$ :

$$
\text { twist }^{*} A_{n}+\operatorname{ch}(s)=\text { twist }^{*} \operatorname{ch}_{f, g}+\operatorname{ch}(s) \equiv \operatorname{ch}_{g, f}+\operatorname{ch}(s) \equiv \operatorname{ch}_{f, g}=A_{n}
$$


The existence of inverses in a monoid is a property, not additional data. This is reflected in the following corollary. Pick arbitrary maps

$$
\nu_{n}: E_{n} \rightarrow E_{n}
$$

and homotopies $h: E_{n} \times I \rightarrow E_{n}$ from $\operatorname{id}_{E_{n}}\left(1 \nu_{n}\right.$ to const.

Example 5.22. We will usually make the following choices:

$$
\nu_{n}: E_{n} \rightarrow \Omega^{2} E_{n+2} \stackrel{\text { flip }}{\longrightarrow} \Omega^{2} E_{n+2} \leftarrow E_{n}
$$

given by exchanging the two path variables. Another possible choice is $\nu_{n}^{\prime}$ : $E_{n} \rightarrow \Omega E_{n+1} \stackrel{\text { inv }}{\longrightarrow} \Omega E_{n+1} \leftarrow E_{n}$ using loop inversion. There is a standard homotopy $h^{\prime}: \operatorname{id} \oplus \nu_{n}^{\prime} \simeq$ const. To define $h$ we precompose $h^{\prime}$ with a homotopy $\nu_{n} \simeq \nu_{n}^{\prime}$ given by 'rotating the axis of reflection:'

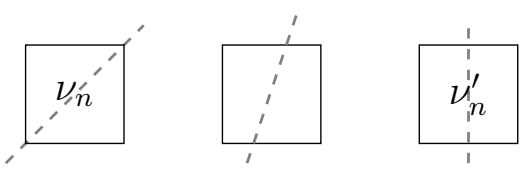

Corollary 5.23. There exist cochains $N_{n} \in \tilde{C}^{n-1}\left(E_{n} ; V\right)$ with the property

$$
\delta N_{n}=-\iota_{n}-\nu_{n}^{*} \iota_{n}
$$

Moreover, we have

$$
N_{n}+\left(\mathrm{id}, \nu_{n}\right)^{*} A_{n}=\operatorname{ch}(h)
$$

Proof. If we define $N_{n}$ according to (53), then a calculation shows that (52) holds, taking 47) and

$$
\delta \operatorname{ch}(h)=\operatorname{ch}\left(\operatorname{id}_{E_{n}}\left(\nu_{n}\right)-\operatorname{ch}(\text { const })=\left(\mathrm{id}, \nu_{n}\right)^{*} \alpha_{n}^{*} \iota_{n}-0\right.
$$

into account.

Of course we have an obvious version of Corollary 5.23 for left inverses, but this leads to different elements $N_{n}^{\prime}$.

Remark 5.24. For any choice of $\left(\nu_{n}, h, N_{n}\right)$ as in Corollary 5.23 the triple $\left(\nu_{n} \circ c_{1},-\omega_{1},-h_{1}+c_{1}^{*} N_{n}\right)$ will represent a (two-sided) inverse in differential cohomology of $\left[c_{1}, \omega_{1}, h_{1}\right]$. This will be clear from the proof of Theorem 5.26 . 


\subsection{Group Structure on Differential Cohomology}

Definition 5.25. Let $x_{1}=\left(c_{1}, \omega_{1}, h_{1}\right), x_{2}=\left(c_{2}, \omega_{2}, h_{2}\right)$ be representatives of elements of $\hat{E}^{n}(M, N)$ as in Definition 3.3. We set

$$
x_{1}+x_{2}:=\left(\alpha_{n} \circ\left(c_{1}, c_{2}\right), \omega_{1}+\omega_{2}, h_{1}+h_{2}+\left(c_{1}, c_{2}\right)^{*} A_{n}\right) .
$$

Also, let $0=($ const, 0,0$)$.

Theorem 5.26. Let $\hat{x}_{1}, \hat{x}_{2} \in \hat{E}^{n}(M, N)$ be represented by triples $x_{1}, x_{2}$ as in 5.25. Then $\hat{x}_{1}+\hat{x}_{2}:=\left[x_{1}+x_{2}\right]$ is well-defined and endows $\hat{E}^{n}(M, N)$ with the structure of an abelian group. The maps a, $I, R$ are group homomorphisms.

Proof. If $\left(C_{1}, H_{1}\right)$ witnesses an equivalence $x_{1}=\left(c_{1}, \omega_{1}, h_{1}\right) \sim\left(c_{1}^{\prime}, \omega_{1}, h_{1}^{\prime}\right)=$ $x_{1}^{\prime}$ and $\left(C_{2}, H_{2}\right)$ witnesses $x_{2}=\left(c_{2}, \omega_{2}, h_{2}\right) \sim\left(c_{2}^{\prime}, \omega_{2}, h_{2}^{\prime}\right)=x_{2}^{\prime}$ in the sense of Definition 3.3, then

$$
Y=\left(\alpha_{n} \circ\left(C_{1}, C_{2}\right), \operatorname{pr}^{*}\left(\omega_{1}+\omega_{2}\right), H_{1}+H_{2}+\left(C_{1}, C_{2}\right)^{*} A_{n}\right)
$$

relates the corresponding sums. For $Y$ obviously restricts on the boundary to $x_{1}+x_{2}, x_{1}^{\prime}+x_{2}^{\prime}$ and it is also easily checked that $\delta\left(H_{1}+H_{2}+\left(C_{1}, C_{2}\right)^{*} A_{n}\right)=$ $\operatorname{pr}^{*}\left(\omega_{1}+\omega_{2}\right)-\left(C_{1}, C_{2}\right)^{*} \alpha_{n}^{*} \iota_{n}$. We conclude that addition is well-defined.

Using Proposition 3.13 (with the canonical homotopies of Subsection 5.3.3), the proofs of associativity, unitality, commutativity, and of inverses reduce immediately to the corresponding parts of Corollaries 5.21, 5.23, For example, let us show that $\left[\nu_{n} \circ c,-\omega,-h+c^{*} N_{n}\right]$ is a right inverse for $[c, \omega, h]$ : select the homotopy $h: \alpha_{n} \circ\left(\mathrm{id}, \nu_{n}\right) \simeq$ const from Corollary 5.23. Then Proposition 3.13 shows that

$$
\begin{aligned}
{[c, \omega, h]+\left[\nu_{n} \circ c,-\omega,-h+c^{*} N_{n}\right] } & =\left[\alpha_{n}\left(\mathrm{id}, \nu_{n}\right) \circ c, 0, c^{*}\left(N_{n}+(\mathrm{id}, \nu)^{*} A_{n}\right)\right] \\
& =\left[\text { const }, 0, c^{*}\left(N_{n}+(\mathrm{id}, \nu)^{*} A_{n}-\int h\right)\right]
\end{aligned}
$$

We conclude by applying Lemma 3.10, since $N_{n}+(\mathrm{id}, \nu)^{*} A_{n}-\int h$ bounds by Corollary 5.23 (in this case even zero). Similarly, $\left[\nu_{n} \circ c,-\omega,-h+N_{n}^{\prime}\right]$ is seen to be a left inverse (at first sight different from the right inverse). But of course the existence of all left and right inverses in any monoid shows that we have a group and that the two inverses coincide. 
The proof that the maps $I, R$ from Definition 3.11 are homomorphisms is trivial. Since we have chosen $A_{n}$ as a reduced cochain, we deduce that $a(\theta)=[$ const, $d \theta, \theta]$ is a homomorphism as well.

\subsection{Bicategory Construction}

We present here an approach to the construction of $\hat{E}^{n}$ in which it is not necessary to make the coherence conditions explicit. The other axioms for a differential extension are also easily verified in this context. The main technical difficulty is the construction of a functorial inverse of the deRham homomorphism, which may systematically be produced in the context of bicategories.

\subsubsection{Inverting the deRham Homomorphism}

Using Example A.3, the map $d: \Omega^{n-1}(M ; V)_{d} \rightarrow \Omega^{n}(M ; V)$ gives rise to a strict symmetric monoidal groupoid $\mathscr{O}^{n}(M)$ which, for varying $M$, piece together a functor

$$
\mathscr{O}^{n}: \operatorname{Man}^{\text {op }} \rightarrow \text { SymMonCat }_{\text {strict }} .
$$

Similarly for $\delta: C^{n-1}(M ; V)_{\delta} \rightarrow Z^{n}(M ; V)$ we obtain

$$
\mathscr{Z}^{n}: \text { Man }^{\text {op }} \rightarrow \text { SymMonCat }_{\text {strict }} \text {. }
$$

Note that the objects of $\mathscr{Z}^{n}(M)$ is the set of cocycles, not all cochains.

The deRham homomorphisms may be interpreted as a strict symmetric monoidal equivalence of categories

$$
\rho_{X}: \mathscr{O}^{n}(M) \stackrel{\sim}{\longrightarrow} \mathscr{Z}^{n}(M),
$$

which are the components of a natural transformation $\mathscr{O}^{n} \rightarrow \mathscr{Z}^{n}$. Every component is an equivalence and we would like to invert $\rho$.

With monoidal transformations as 2-arrows, SymMonCat forms a strict 2-category. We will view Man as a 2-category with only identity 2-arrows. Then $\rho$ is a strict transformation between the two strict functors $\mathscr{O}^{n}, \mathscr{Z}^{n}$ of bicategories.

Lemma 5.27. Suppose $\mathscr{C}$ is a category, considered trivially as a bicategory. Let

$$
F, G: \mathscr{C} \longrightarrow \text { SymMonCat }
$$


be strict functors of bicategories and $u: F \Rightarrow G$ a strict transformation which has the property that all of the functors $u_{X}: F X \rightarrow G X$ are equivalences. Then there exists a weak transformation $v: G \Rightarrow F$ such that uv and vu are the identity functors up to modifications.

Proof. By Leinster, 1.2.14 we may place each $u_{X}$ in a monoidal adjoint equivalence $\left(u_{X}, v_{X}, \varepsilon_{X}, \eta_{X}\right)$ - meaning that we have an adjoint equivalence in which $v_{X}$ is a monoidal functor and $\varepsilon_{X}, \eta_{X}$ are monoidal transformations.

It remains to check that the monoidal functors $\left(v_{X}, \mu_{X}\right)$ depend functorially on $X$ up to coherent 2-cells $v_{f}:(F f, \phi) \circ\left(v_{X}, \nu_{X}\right) \Rightarrow\left(v_{Y}, \nu_{Y}\right) \circ(G f, \gamma)$ for $f: X \rightarrow Y$ in $\mathscr{C}$. The monoidal transformation $v_{f}$ is defined as the 2-cell

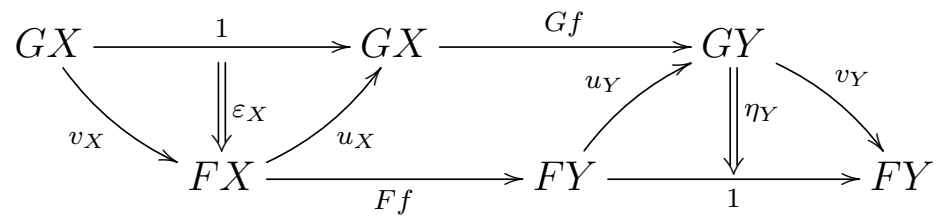

Note that the middle diagram commutes. The coherence conditions follow from the zig-zag equations for $\varepsilon_{X}, \eta_{X}$.

We may thus find a weak transformtion, unique up to modifications,

$$
\tau: \mathscr{Z}^{n} \Longrightarrow \mathscr{O}^{n},
$$

inverse to $\rho$ in the sense of the preceding lemma.

Proposition 5.28. It is possible to lift the Chern character transformation ch : $\left[M, E_{n}\right] \rightarrow H^{n}(M ; V)$ to a braided monoidal functor

$$
\operatorname{Map}\left(M, E_{n}\right) \rightarrow \mathscr{Z}^{n}(M),
$$

natural in $M$, i.e. to a strict transformation $\operatorname{Map}\left(-, E_{n}\right) \rightarrow \mathscr{Z}^{n}$.

Proof. This is Theorem 5.19 applied to $X=M^{+}$and where we observe that ch takes values in the full subcategory $\mathscr{Z}^{n}$.

Composing this transformation with the weak inverse $\tau$ of the deRham transformation, we obtain a weak transformation

$$
\operatorname{ch}: \operatorname{Map}\left(-, E_{n}\right) \Longrightarrow \mathscr{O}^{n} \text {. }
$$


What this means explicitly it that we are given monoidal functors

$$
\operatorname{ch}_{M}: \operatorname{Map}\left(M, E_{n}\right) \longrightarrow \mathscr{O}^{n}(M), \quad M \in \text { Man, }
$$

that is, a functor $\mathrm{ch}_{M}$ together with a natural transformation, denoted $\mathrm{ch}_{M}^{+}$: $\operatorname{ch}_{M} \circ \ominus \rightarrow+\circ\left(\operatorname{ch}_{M} \times \operatorname{ch}_{M}\right)$, and a map $\operatorname{ch}_{M}$ (const) $\rightarrow 0$ which may be chosen to be zero (since the fundamental cocycles are reduced). Also, for every smooth map $f: M \rightarrow N$ we have a map

$$
\operatorname{ch}_{f}: E_{n}^{N} \longrightarrow \Omega^{n-1}(M ; V)_{d}
$$

with

$$
d\left(\operatorname{ch}_{f}(c)\right)=f^{*} \operatorname{ch}_{N}(c)-\operatorname{ch}_{M}(c \circ f) \quad \text { for all } c: N \rightarrow E_{n}
$$

$f^{*} \operatorname{ch}_{N}(H)+\operatorname{ch}_{f}\left(H_{0}\right)=\operatorname{ch}_{f}\left(H_{1}\right)+\operatorname{ch}_{M}\left(H \circ\left(f \times \operatorname{id}_{I}\right)\right)$ for a homotopy $H: H_{0} \simeq H_{1}$

where $H: N \times I \rightarrow E_{n}$. The coherence conditions for $M \stackrel{f}{\rightarrow} N \stackrel{g}{\rightarrow} O \stackrel{c}{\longrightarrow} E_{n}$ amount to

$$
\begin{aligned}
\operatorname{ch}_{g f}(c) & =\operatorname{ch}_{f}(c \circ g)+f^{*} \operatorname{ch}_{g}(c), \\
\operatorname{ch}_{\text {id }} & =0 .
\end{aligned}
$$

The compatibility condition for the $\iota_{n}$ ensure that for a map $h: M \rightarrow E_{n-1}$, viewed as a homotopy $H: M \times I \rightarrow E_{n}$ from const to const, we have

$$
\operatorname{ch}_{M}(h)=\operatorname{ch}_{M}(H) \in \Omega^{n-1}(M ; V)_{d} .
$$

\subsubsection{Construction of Differential Refinements}

Consider the equivalence relation $\sim$ on the set $\mathscr{E}^{n}(M)=E_{n}^{M} \times \Omega^{n-1}(M ; V)_{d}$ given by $\left(c_{1}, \omega_{1}\right) \sim\left(c_{2}, \omega_{2}\right)$ iff there exists a homotopy $H: c_{1} \rightarrow c_{2}$ with

$$
\operatorname{ch}_{M}(H)=\omega_{1}-\omega_{2} .
$$

Reflexivity, symmetry, and transitivity of ' $\sim$ ' follow from the following functional properties of $\operatorname{ch}_{M}$ : that it preserves the identity, inverses of isomorphisms, and composition. We may now define

$$
\hat{E}^{n}(M)=\mathscr{E}^{n}(M) / \sim
$$


For a smooth map $M \stackrel{f}{\rightarrow} N$ we let

$$
\hat{E}^{n}(f): \hat{E}^{n}(N) \longrightarrow \hat{E}^{n}(M), \quad[c, \omega] \mapsto\left[c \circ f, f^{*} \omega+\operatorname{ch}_{f}(c)\right]
$$

This is well-defined by $(56)$ and yields a functor $\hat{E}^{n}:$ Man $^{\text {op }} \longrightarrow$ Set by (57) and (58). The group structure is given by

$$
\begin{aligned}
{\left[c_{1}, \omega_{1}\right]+\left[c_{2}, \omega_{2}\right] } & =\left[c_{1} \ominus c_{2}, \omega_{1}+\omega_{2}+\operatorname{ch}_{M}^{+}\left(c_{1}, c_{2}\right)\right], \\
0 & =[\text { const }, 0] .
\end{aligned}
$$

Define natural transformations

$$
\begin{aligned}
\Omega^{n-1}(M ; V)_{d} \stackrel{a}{\rightarrow} \hat{E}^{n}(M), & a(\omega)=[\text { const, } \omega] \\
\hat{E}^{n}(M) \stackrel{I}{\rightarrow} E^{n}(M), & I[c, \omega]=[c] \in\left[M, E_{n}\right]=E^{n}(M) \\
\hat{E}^{n}(M) \stackrel{R}{\longrightarrow} \Omega^{n}(M ; V)_{d=0}, & R[c, \omega]=\operatorname{ch}(c)+d \omega
\end{aligned}
$$

The map $R$ is well-defined, again by functoriality of $\mathrm{ch}_{M}$.

Proposition 5.29. $\hat{E}^{n}$ is a differential cohomology theory in the sense of [BS10]. That is, we have a commutative diagram with an exact row:

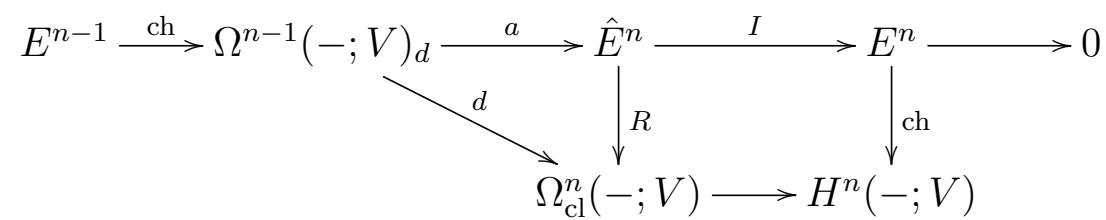

Proof. The commutativity is clear. If $I[c, \omega]=0$ there exists a homotopy $H: c \rightarrow$ const so that $[c, \omega]=\left[\right.$ const, $\left.\omega+\operatorname{ch}_{M}(H)\right]=a\left(\omega+\operatorname{ch}_{M}(H)\right)$. Also $I \circ a=0$. If $a(\omega)=$ [const, 0 ] then there exists $H$ : const $\rightarrow$ const with $\operatorname{ch}_{M}(H)=\omega$. Conversely, a map $c: M \rightarrow E_{n-1}$ may be viewed as a homotopy $H:$ const $\simeq$ const $: M \rightarrow E_{n}$. Then $a(\operatorname{ch}(h))=[$ const, $\operatorname{ch}(h)]=[$ const, 0$]$, using $H$ and the compatibility (16) of the $\iota_{n}$.

\section{INTEGRATION MAPS}

In this chapter we will develop the theory of integration maps

$$
\int: \hat{E}^{n+1}\left(M \times S^{1}\right) \rightarrow \hat{E}^{n}(M)
$$


in differential cohomology. These will be important because they relate different degrees: later, products will initially only be defined in even degrees. It will then be our strategy to 'transport' this structure to odd degrees by demanding compatibility with the integration maps. Subsection 6.2.5 on multiple integrals will help us clarify well-definedness for extensions of this sort.

In the case of generalized cohomology, the underlying spectrum is at first related to 'reduced' integration maps (the suspension) and may then be modified to an 'absolute' version (Remark 6.14). Similarly, integration in differential cohomology may be understood explicitly only on pairs and is then extended to the absolute case.

\subsection{Slant Products and Integration over the Fiber}

\subsubsection{Cochains}

The Eilenberg-Zilber map may be used to construct the integration (or slant product) of cochains. For a pair of simplicial sets $A \subset X$ let

$$
C^{*}(X, A)=\operatorname{Hom}_{\mathbb{Z}}\left(C_{*}(X) / C_{*}(A), \mathbb{R}\right)
$$

denote the real cochain complex obtained by dualizing the Moore complex (note that the reals are not reflected in the notation of this cochain complex; the Moore chain complex, however, is conventionally taken over the integers). The differential is usually taken to be 'pullback along the Moore differential'. The introduction of a sign $(-1)^{\text {degree }}$ would make this an internal hom, but we will stick to the more traditional convention.

Definition 6.1. Let $X, Y$ be simplicial sets and suppose $e \in C_{k}(Y)$. We define natural homomorphisms $\int_{e}: C^{*}(X \times Y) \longrightarrow C^{*-|e|}(X)$ as follows: a cochain $u: C_{*}(X \times Y) \rightarrow \mathbb{R}$ is mapped to the composition

$$
C_{*-k}(X) \stackrel{\operatorname{id} \otimes e}{\longrightarrow} C_{*-k}(X) \otimes C_{k}(Y) \stackrel{E Z}{\longrightarrow} C_{*}(X \times Y) \stackrel{u}{\longrightarrow} \mathbb{R} .
$$

Here, $E Z$ denotes the Eilenberg-Zilber map from Definition 5.2 .

Lemma 6.2. The integration maps are $\mathbb{R}$-linear.

(i) The integration maps are natural in the sense that

$$
\int_{e}\left(f \times \operatorname{id}_{Y}\right)^{*} u=f^{*}\left(\int_{e} u\right), \quad \int_{f_{*} e} u=\int_{e}\left(\operatorname{id}_{X} \times f\right)^{*} u .
$$


(ii) We have Stokes' formula $\int_{e} \delta u=\delta \int_{e} u-(-1)^{|u|+|e|} \int_{\partial e} u$.

Proof. Linearity is trivial, (i) follows from the naturality of the EilenbergZilber maps, while (ii) is just the fact that $E Z$ is a chain map.

Naturality implies that integration descends to relative cochain complexes:

$$
\int_{e}: C^{*}((X, A) \times Y) \rightarrow C^{*-|e|}(X, A) .
$$

Remark 6.3. It would be more precise to call $\int_{e}$ integration from the right. There is an obvious version from the left (used for instance for integrating the pullback $\int_{I} H^{*} \iota$ along homotopies - whose time interval we conventionally take on the left):

$$
\int_{e}^{\prime}: C^{*}(Y \times X) \rightarrow C^{*-|e|}(X) .
$$

Usually, there should be no confusion if we denote this by the symbol $\int$ as well. If care needs to be taken, we will include the slash for integration from the left. It is important to distinguish between these two integration maps, especially if $X=Y$, because there is a sign involved: if $\tau: X \times Y \rightarrow Y \times X$ denotes the twist, then the symmetry properties of the Eilenberg-Zilber map imply

$$
\int_{e}^{\prime} u=(-1)^{(|u|-|e|) \cdot|e|} \int_{e} \tau^{*} u .
$$

Stokes' formula on the left reads

$$
\int_{e}^{\prime} \delta u=\int_{\partial e}^{\prime} u+(-1)^{e} \delta \int_{e}^{\prime} u .
$$

By passing to singular sets, we may use the above constructions for spaces $A \subset X$ and $Y$.

Remark 6.4. We will primarily take the CW-complex $Y=S^{1}$ and the canonical 1-chain $e \in C_{1}\left(\operatorname{sing} S^{1}\right)$, which is the adjoint of the canonical projection $\left|\Delta^{1}\right| \rightarrow S^{1}$. Since such integrals will appear a lot we shall omit the reference to $S^{1}$ when integrating over the circle. Stokes' formula simplifies to

$$
\int \delta u=\delta \int u, \quad \int^{\prime} \delta u=-\delta \int^{\prime} u
$$


Remark 6.5. In the case of manifolds with corners, we remark that $E Z$ carries the complex of smooth chains to itself. If $e$ is a smooth chain, we therefore have a version of $(59)$ for smooth cochains (take the smooth singular sets for $X$ and $Y$ in Definition 6.1).

\subsubsection{Differential Forms}

We have used the 'integral' notation for the slant product of cochains in the previous section because it generalizes the integral along the fiber of differential forms $\omega \in \Omega^{n}(M \times I)$ (for the proof cf. [HS05, Lemma 3.15] or the remark below).

Definition 6.6. Given a differential form $\omega \in \Omega^{n+1}(M \times I)$, let $\left(U_{\alpha}, \phi_{\alpha}\right)_{\alpha \in A}$ be an atlas of $M$ and $\left(\chi_{\alpha}\right)$ a subordinate partition of unity. Using the product atlas $\left(\phi_{\alpha} \times \operatorname{id}_{I}\right)_{\alpha \in A}$ at $(x, t) \in U_{\alpha} \times I$ we may write:

$\omega_{(x, t)}=\sum_{i_{1}<\ldots<i_{n}} \omega_{i_{1}, \ldots, i_{n}}^{\alpha}(x, t) d \phi_{\alpha}^{i_{1}} \cdots d \phi_{\alpha}^{i_{n}} \wedge d t+\sum_{i_{1}<\ldots<i_{n+1}} \tilde{\omega}_{i_{1}, \ldots, i_{n+1}}^{\alpha}(x, t) d \phi_{\alpha}^{i_{1}} \cdots d \phi_{\alpha}^{i_{n+1}}$

In accordance with [BT82, p.61], we define the integral of $\omega$ along $I$ (on the right) by

$$
\left(\int_{I} \omega\right)_{x}:=\sum_{\alpha} \chi_{\alpha}(x) \cdot \sum_{i_{1}<\ldots<i_{n}}\left(\int_{0}^{1} \omega_{i_{1}, \ldots, i_{n}}^{\alpha}(x, t) d t\right)\left(d \phi_{\alpha}^{i_{1}}\right)_{x} \cdots\left(d \phi_{\alpha}^{i_{n}}\right)_{x} .
$$

The above definition is independent of the choices $\left(U_{\alpha}, \phi_{\alpha}\right)$ and $\chi_{\alpha}$; for the tedious verification we refer to [BT82]. We have naturality in Definition 6.6 in the sense that

$$
\int_{I}\left(f \times \mathrm{id}_{I}\right)^{*} \omega=f^{*}\left(\int_{I} \omega\right), \quad f: M^{\prime} \rightarrow M \text { smooth. }
$$

In view of (6), this leads to a relative integration map for forms. Precomposing with pullback along $\operatorname{id}_{(M, N)} \times p$ for the canonical map $p: I \rightarrow S^{1}$, we obtain integration over $S^{1}$ (again omitting reference to $S^{1}$ in the notation)

$$
\int: \Omega^{n+1}\left((M, N) \times S^{1}\right) \rightarrow \Omega^{n}(M, N) .
$$

In Definition 6.6 we sent forms that have no $d t$-component to zero. For the projection $\operatorname{pr}_{1}:(M, N) \times S^{1} \rightarrow(M, N)$ we therefore have

$$
\int \operatorname{pr}_{1}^{*} \omega=0 .
$$


Remark 6.7. A differential form $\omega \in \Omega^{n+1}\left(M \times S^{1}\right)$ may be viewed as a cochain $u$, which we usually also denote by $\omega$. This causes no confusion in connection with the integration map since the cochain associated to $\int_{S^{1}} \omega$ and the cochain $\int_{S^{1}} u$ coincide on behalf of the calculation for $M=\Delta^{n}$ :

$\int_{\Delta^{n}} \int_{S^{1}} \omega=\int_{\Delta^{n} \times S^{1}} \omega=\sum_{i=0}^{n}(-1)^{i} \int_{\Delta^{n+1}} h_{i}^{*} \omega=u\left(E Z\left(\Delta^{n} \otimes S^{1}\right)\right)=\left(\int_{S^{1}} u\right)\left(\Delta^{n}\right)$.

The signs appear due to orientations.

\subsubsection{E-cocycles}

As above, fix a spectrum $E_{n}$ with structure maps $\varepsilon_{n}: E_{n} \wedge S^{1} \rightarrow E_{n+1}$ whose adjoints $E_{n} \rightarrow \Omega E_{n+1}$ are homeomorphisms.

Definition 6.8. For a map of pairs $c:\left(M \times S^{1}, M \times 1\right) \rightarrow\left(E_{n+1}, *\right)$ there is a unique map $\int c: M \rightarrow E_{n}$ with

$$
\varepsilon_{n} \circ\left(\int c \wedge \mathrm{id}_{S^{1}}\right)=c
$$

namely the map $\left(\varepsilon_{n}^{\text {adj }}\right)^{-1} \circ c^{\text {adj }}$.

We again have naturality

$$
\int\left(f \wedge \operatorname{id}_{S^{1}}\right)^{*} c=f^{*}\left(\int c\right) .
$$

Recall that in (16) we have chosen the fundamental cocycles $\iota_{n}$ compatible with the structure maps. Using Lemma 6.2 (i), this implies that Definitions 6.1 and 6.8 are compatible in the sense that

$$
\left(\int c\right)^{*} \iota_{n}=\left(\int c\right)^{*} \int \varepsilon_{n}^{*} \iota_{n+1}=\int\left(\int c \wedge \mathrm{id}_{S^{1}}\right)^{*} \varepsilon_{n}^{*} \iota_{n+1}=\int\left(c^{*} \iota_{n}\right) .
$$

\subsection{Integration in Differential Cohomology}

For integration in differential cohomology to be linear, we need to compare the additive structure in neighboring degrees. This is the reason for having worked above in $\mathbf{2 B r M o n C a t}$. We again exhibit the coherence conditions inherent in Theorem 5.19 . 


\subsubsection{Loop Spectra}

For varying degrees $n$ the functors from 29

$$
\left(\Pi_{1}\right)_{-n}: \text { coSpt } \rightarrow \text { 2BrMonCat }
$$

are related. The notation from Subsection 4.3.1 can be confusing when dealing with loop spectra $\Omega E=\operatorname{Hom}\left(S^{1}, E\right)$. Recall that $\left(\Pi_{1}\right)_{-n} E$ denotes the fundamental groupoid $\Pi_{1} E_{n}$ along with two monoidal structures

$$
\begin{array}{lll}
\text { (1) by writing } & E_{n} \simeq \Omega E_{n+1}, \\
\ominus & \text { by writing } & E_{n} \simeq \Omega^{2} E_{n+2},
\end{array}
$$

and a monoidal structure $e$ on the identity functor $\left(\Pi_{1} E_{n}, \Phi\right) \rightarrow\left(\Pi_{1} E_{n}, \ominus\right)$. Define forgetful functors

$$
q^{1}, q^{2}: \text { 2BrMonCat } \rightarrow \text { MonCat }, \quad(\mathscr{C}, \oplus, \ominus, e) \mapsto(\mathscr{C}, \oplus),(\mathscr{C}, \ominus) .
$$

The structure maps on $E$ determine a functor $\Pi_{1} \Omega E_{n+1} \rightarrow \Pi_{1} E_{n}$ which by Proposition A.13 inherits a unique monoidal structure turning the following into a diagram in MonCat

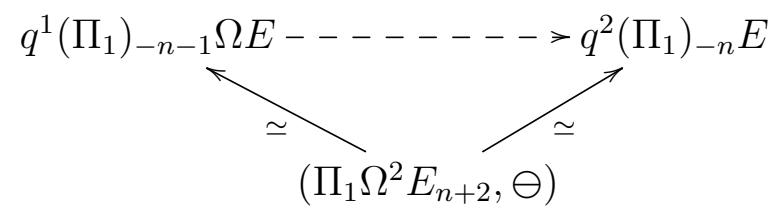

Here, the two equivalences are monoidal essentially by definition of the corresponding monoidal structures on $\left(\Pi_{1}\right)_{-*}$. For example, for the left hand diagonal map, recall that the symbol $q^{1}\left(\Pi_{1}\right)_{-n-1} \Omega E$ denotes the category $\Pi_{1} \Omega E_{n+1}$ with the monoidal structure ' $\Phi$ ' given by the equivalence

$$
\left(\Pi_{1}\left(\Omega E_{n+1}\right), \Phi\right) \stackrel{\simeq}{\longleftarrow}\left(\Pi_{1} \Omega\right)\left(\Omega E_{n+2}\right) .
$$

By definition of the structure maps of a loop spectrum, the loop space in $\left(\Pi_{1} \Omega\right)$ on the right refers to the coordinate obtained by writing $E_{n+1} \cong$ $\Omega E_{n+2}$. The monoidal structure ' $\Phi$ ' on the right corresponds therefore to ' $\ominus$ ' in $\left(\Pi_{1} \Omega^{2}\right) E_{n+2}$. More precisely, Propositions A.13 and A.14 provide the structure of monoidal functor on the dotted arrow in

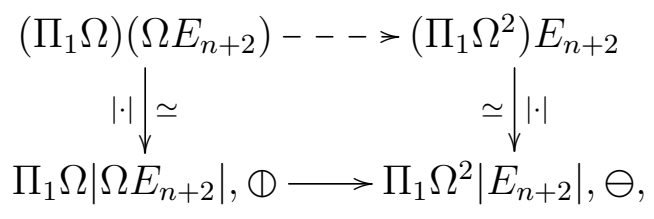


where the vertical functors are monoidal by definition. The lower horizontal functor is induced by the canonical map $|\Omega X| \rightarrow \Omega|X|$.

Proposition 6.9. Given a morphism $f: E \rightarrow F$ in coSpt, we have a commutative diagram

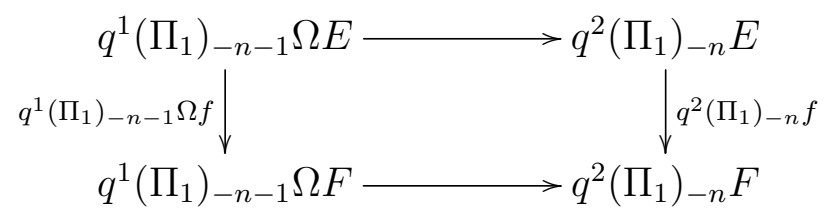

Proof. According to the uniqueness part of Proposition A.13 it suffices to prove equality upon precomposing with $\left(\Pi_{1} \Omega^{2} E_{n+2}, \ominus\right) \stackrel{\simeq}{\longrightarrow} q^{1}\left(\Pi_{1}\right)_{-n} E$ which then boils down to the definition of monoidal structure on the functors $q^{1}\left(\Pi_{1}\right)_{-n-1} \Omega f$ and $q^{2}\left(\Pi_{1}\right)_{-n} f$.

\subsubsection{Compatibility of ch with Eckmann-Hilton}

Applying Proposition 6.9 to 40 exhibits the commutativity in MonCat of the upper square in the diagram

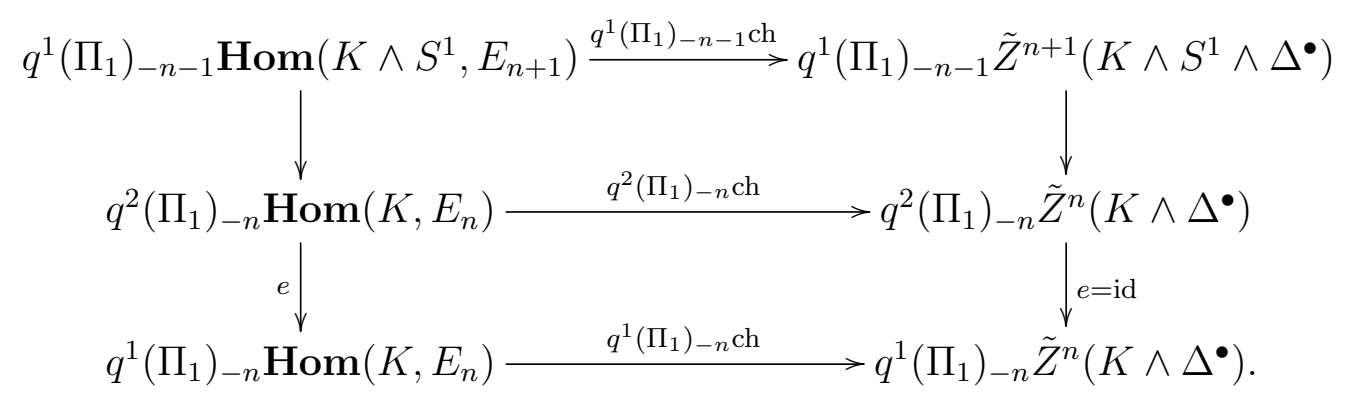

The lower square commutes since $\left(\Pi_{1}\right)_{-n}$ ch is a morphism in 2 BrMonCat. Making the transition to spaces by using the above diagram for $K=\operatorname{sing}(X)$, $\left(\operatorname{sing}\left(E_{n}\right), \operatorname{sing}\left(\varepsilon_{n}\right)\right)$ and by using the maps 45, 46) we obtain a commuta- 
tive diagram in MonCat

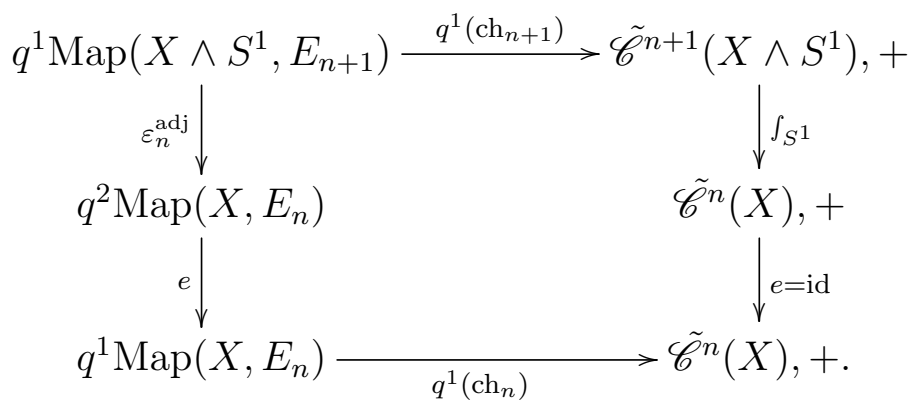

Recall that the Eckmann-Hilton isomorphisms (homotopies in this case) $e: q^{2} \operatorname{Map}\left(X, E_{n}\right) \rightarrow q^{1} \operatorname{Map}\left(X, E_{n}\right)$ are given by writing $E_{n} \approx \Omega^{2} E_{n+2}$ and the formula 25 . These are the usual homotopies relating the two possible additions ' $\left(1\right.$ ' (which yields $\alpha_{n}$ in the notation of Corollary 5.21), ' $\ominus$ ' on a double loop space.

Corollary 6.10. Let $\varphi_{n}:\left(E_{n} \times E_{n}\right) \wedge S^{1} \rightarrow E_{n+1} \times E_{n+1}$ denote the 'product of structure maps' and $e: E_{n}^{\times 2} \times I \rightarrow E_{n}$ the Eckmann-Hilton homotopy. Then

$$
\int_{S^{1}}\left(\varphi_{n}\right)^{*} A_{n+1} \equiv \operatorname{ch}(e)+A_{n} \quad \bmod \operatorname{im}(d)
$$

Proof. Set $X=E_{n} \times E_{n}$ in the previous commuting diagram in MonCat. The two ways around yield two monoidal functors (whose composition is recalled in (107)) that agree. In particular, their 'extra monoidal data' agrees at the objects $\operatorname{pr}_{1}^{\text {adj }}, \operatorname{pr}_{2}^{\text {adj }}: X \wedge S^{1} \rightarrow E_{n+1}$ (which are adjoint to the two projections $\operatorname{pr}_{1}, \mathrm{pr}_{2}: E_{n} \times E_{n} \rightarrow \Omega E_{n+1} \approx E_{n}$ ). This yields $\int \mathrm{ch}_{\mathrm{pr}_{1}^{\text {adj }}{ }_{\text {, }}{ }_{2}^{\text {adj }}} \equiv$ $\operatorname{ch}(e)+\operatorname{ch}_{\mathrm{pr}_{1}, \mathrm{pr}_{2}}$, which in the notation of Corollary 5.21 reads 66.

\subsubsection{Construction on Pairs}

Definition 6.11. By combining the three integration maps above, we obtain

$$
\int: \hat{E}^{n+1}\left(M \times S^{1}, M \times 1\right) \rightarrow \hat{E}^{n}(M), \quad[c, \omega, h] \mapsto\left[\int c, \int \omega, \int h\right] .
$$

This triple represents a differential cohomology class: combining (60) and 65), we have

$$
\delta \int h=\int \delta h=\int \omega-\int\left(c^{*} \iota_{n}\right)=\int \omega-\left(\int c\right)^{*} \iota_{n} .
$$


Moreover, the class of this triple is well-defined: if $(H, C)$ witnesses an equivalence $(c, \omega, h) \sim\left(c^{\prime}, \omega, h^{\prime}\right)$, then $\left(\int H, \int C\right)$ yields the necessary equivalence.

Proposition 6.12. The integration map in differential cohomology is a natural transformation $\hat{E}^{n+1}\left(-\times S^{1},-\times 1\right) \rightarrow \hat{E}^{n}(-)$. In particular, integration is linear and natural in the sense of $\int\left(f \times \operatorname{id}_{S^{1}}\right)^{*} \hat{x}=f^{*}\left(\int \hat{x}\right)$.

Proof. Naturality follows from the naturality of the integration map for forms, cochains, and E-cocycles. For linearity, let $\left(c_{1}, \omega_{1}, h_{1}\right),\left(c_{2}, \omega_{2}, h_{2}\right)$ represent classes $\hat{x}_{1}, \hat{x}_{2}$ in $\hat{E}^{n+1}\left(M \times S^{1}, M \times 1\right)$. We compute

$$
\begin{aligned}
& \int\left(\hat{x}_{1}+\hat{x}_{2}\right)=\left[\int \alpha_{n+1}\left(c_{1}, c_{2}\right), \int\left(\omega_{1}+\omega_{2}\right), \int\left(h_{1}+h_{2}+\left(c_{1}, c_{2}\right)^{*} A_{n+1}\right)\right] \\
& \int \hat{x}_{1}+\int \hat{x}_{2}=\left[\alpha_{n}\left(\int c_{1}, \int c_{2}\right), \int \omega_{1}+\int \omega_{2}, \int\left(h_{1}+h_{2}\right)+\left(\int c_{1}, \int c_{2}\right)^{*} A_{n}\right]
\end{aligned}
$$

Using (63) and (64) we see that

$$
\int \alpha_{n+1}\left(c_{1}, c_{2}\right)=\left(\int c_{1}, \int c_{2}\right)^{*} \int \alpha_{n+1}\left(\varepsilon_{n}, \varepsilon_{n}\right)
$$

is the pullback along $\left(\int c_{1}, \int c_{2}\right)$ of the starting point of the Eckmann-Hilton homotopy from Corollary 6.10. Combining Lemma 3.10 and Proposition 3.13, we see that in order to prove equality of the differential cohomology classes above it suffices to show that

$$
\int\left(c_{1}, c_{2}\right)^{*} A_{n+1}-\operatorname{ch}\left(\left(\int c_{1}, \int c_{2}\right)^{*} h\right) \equiv\left(\int c_{1}, \int c_{2}\right)^{*} A_{n}
$$

modulo coboundaries. But, using (63), this is just the pullback along $\left(\int c_{1}, \int c_{2}\right)$ of $(66)$.

Proposition 6.13. The integration map commutes with the maps a, $R, I$.

Proof. Straightforward from the definitions.

\subsubsection{Integration Maps}

We have developed differential cohomology for pairs in order to be able to define rigorously the integration map $\int: \hat{E}^{n+1}\left(M \times S^{1}, M \times 1\right) \rightarrow \hat{E}^{n}(M)$. In this subsection we will use our 'pair' integration map to define an 'absolute' integration map

$$
\int: \hat{E}^{n+1}\left(M \times S^{1}\right) \rightarrow \hat{E}^{n}(M) .
$$


Remark 6.14. To motivate our definition, let us consider the case of (generalized) cohomology [BS10]. Let

$$
\begin{aligned}
& i: M=M \times 1 \subset M \times S^{1}, \\
& j:\left(M \times S^{1}, \emptyset\right) \rightarrow\left(M \times S^{1}, M \times 1\right), \\
& \operatorname{pr}_{1}: M \times S^{1} \rightarrow M
\end{aligned}
$$

We have a long exact sequence

$$
\cdots \rightarrow E^{n}\left(M \times S^{1}\right) \stackrel{i^{*}}{\rightarrow} E^{n}(M) \stackrel{\delta}{\rightarrow} E^{n+1}\left(M \times S^{1}, M \times 1\right) \stackrel{j^{*}}{\rightarrow} E^{n+1}\left(M \times S^{1}\right) \rightarrow \cdots
$$

Since $i$ is a section of the projection $\mathrm{pr}_{1}$, the map $i^{*}$ is surjective, so $\delta=0$ and $j^{*}$ is injective. For any $x \in E^{n+1}\left(M \times S^{1}\right)$ we therefore have a unique element $y \in E^{n+1}\left(M \times S^{1}, M \times 1\right)$ with $j^{*} y=x-\operatorname{pr}_{1}^{*} i^{*} x$. Define

$$
\int x:=\int y \in E^{n}(M),
$$

where the integral on the right is given by suspension $E^{n+1}\left(M \times S^{1}, M \times 1\right) \cong$ $\tilde{E}^{n+1}\left(M_{+} \wedge S^{1}\right) \cong \tilde{E}^{n}\left(M_{+}\right)$. These definitions piece together linear maps

$$
\int: E^{n+1}\left(M \times S^{1}\right) \rightarrow E^{n}(M)
$$

which fit into commutative diagrams

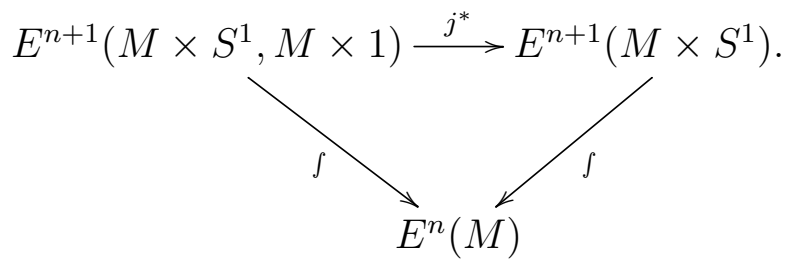

Clearly, $\int \operatorname{pr}_{1}^{*} x=0$ for all $x \in E^{n+1}(M)$.

Using a similar procedure, we now define maps (67).

Theorem 6.15. Suppose $\int: \hat{E}^{n+1}\left(-\times S^{1},-\times 1\right) \rightarrow \hat{E}^{n}$ is an integration on pairs as in Proposition 6.12 satisfying also Proposition 6.13. Then there exist a unique natural transformation (linear)

$$
\int: \hat{E}^{n+1}\left(M \times S^{1}\right) \rightarrow \hat{E}^{n}(M)
$$

with the following properties: 
(i) The composition of (68) with $j^{*}$ is integration on pairs (Definition 6.11):

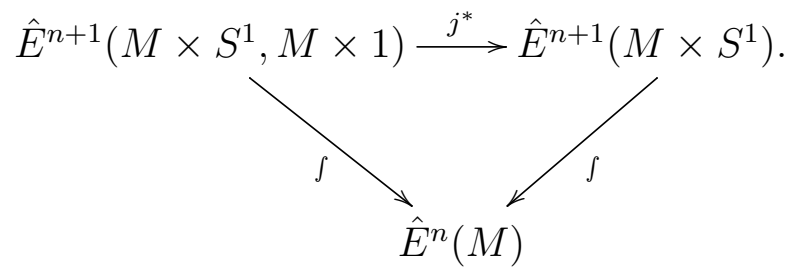

(ii) $\int \mathrm{pr}_{1}^{*}=0$.

Proof. To prove uniqueness, suppose $\int_{1}, \int_{2}$ are two transformations satisfying (i) and (ii) and let $\hat{x} \in \hat{E}^{n+1}\left(M \times S^{1}\right)$. According to Theorem 3.15, $\hat{x}-\operatorname{pr}_{1}^{*} i^{*} \hat{x}$ may be written as $j^{*} \hat{y}$ for some $\hat{y} \in \hat{E}^{n+1}\left(M \times S^{1}, M \times 1\right)$. Then

$$
\int_{1} \hat{x} \stackrel{(i i)}{=} \int_{1}\left(\hat{x}-\operatorname{pr}_{1}^{*} i^{*} \hat{x}\right) \stackrel{(i)}{=} \int \hat{y} \stackrel{(i)}{=} \int_{2}\left(\hat{x}-\operatorname{pr}_{1}^{*} i^{*} \hat{x}\right) \stackrel{(i i)}{=} \int_{2} \hat{x}
$$

Conversely, we define a map $(68)$ as follows: for each $\hat{x} \in \hat{E}^{n+1}\left(M \times S^{1}\right)$ write $\hat{x}-\operatorname{pr}_{1}^{*} i^{*} \hat{x}=j^{*} \hat{y}$ for some $\hat{y} \in \hat{E}^{n+1}\left(M \times S^{1}, M \times 1\right)$. Set

$$
\int \hat{x}:=\int \hat{y}
$$

This definition clearly satisfies (i) and (ii), so it remains to check that (69) is well-defined. Using the linearity, it suffices to show the implication $j^{*} \hat{y}=$ $0 \Rightarrow \int \hat{y}=0$. Consider the following commutative diagram with exact rows:

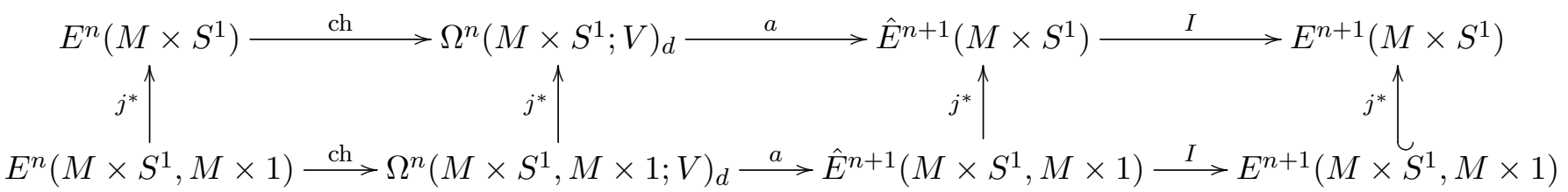

Since $0=I j^{*} \hat{y}=j^{*} I \hat{y}$ and $j^{*}: E^{n+1}\left(M \times S^{1}, M \times 1\right) \rightarrow E^{n+1}\left(M \times S^{1}\right)$ is injective we may write $\hat{y}=a(\theta)$. The class $j^{*} \theta$ lies in the kernel of $a$ and therefore $j^{*} \theta=\operatorname{ch}(t)$ for some $t \in E^{n}\left(M \times S^{1}\right)$. Next, $t-\operatorname{pr}_{1}^{*} i^{*} t$ lies in the kernel of $i^{*}$, so we may write $t-\operatorname{pr}_{1}^{*} i^{*} t=j^{*} s$ for some $s \in E^{n}\left(M \times S^{1}, M \times 1\right)$. Remark 6.14 implies

$$
\int_{S^{1}} s=\int_{S^{1}} j^{*} s=\int_{S^{1}} t-\int_{S^{1}} \operatorname{pr}_{1}^{*} i^{*} t=\int_{S^{1}} t .
$$


Because the Chern character is a transformation of cohomology theories, we have $\int_{S^{1}} \operatorname{ch}(s)=\operatorname{ch}\left(\int_{S^{1}} s\right)=\operatorname{ch}\left(\int_{S^{1}} t\right)=\int_{S^{1}} \operatorname{ch}(t)$. Thus

$$
\int_{S^{1}} \hat{y}=a\left(\int_{S^{1}} \theta\right)=a\left(\int_{S^{1}} j^{*} \theta\right)=a\left(\int_{S^{1}} \operatorname{ch}(t)\right)=a\left(\operatorname{ch}\left(\int_{S^{1}} s\right)\right)=0 .
$$

This proves well-definedness. From this one concludes linearity, using that $i^{*}, \operatorname{pr}_{1}^{*}$ are linear maps in differential cohomology. Similarly, naturality in Proposition 6.12 implies the naturality of (68).

Proposition 6.16. The integration maps $(68)$ commute with a, $R, I$.

Proof. This follows easily from Proposition 6.13 and the previous theorem: If $\hat{x} \in \hat{E}^{n+1}\left(M \times S^{1}\right)$ write $j^{*} \hat{y}=\hat{x}-\operatorname{pr}_{1} i^{*} \hat{x}$. Then we have

$$
I\left(\int \hat{x}\right)=I\left(\int \hat{y}\right) \stackrel{6.13}{=} \int I \hat{y} \stackrel{(i)}{=} \int j^{*} I \hat{y}=\int I\left(j^{*} \hat{y}\right)=\int I\left(\hat{x}-\operatorname{pr}_{1} i^{*} \hat{x}\right) \stackrel{(i i)}{=} \int I \hat{x}
$$

and similarly for $R$. If $\theta \in \Omega^{n}\left(M \times S^{1} ; V\right)$ write $j^{*} \eta=\theta-\operatorname{pr}_{1}^{*} i^{*} \theta$. Then

$$
\int a(\theta) \stackrel{(i i)}{=} \int a\left(j^{*} \eta\right) \stackrel{(i)}{=} \int a(\eta) \stackrel{6.13}{=} a\left(\int \eta\right)=a\left(\int j^{*} \eta\right)=a\left(\int \theta\right) .
$$

\subsubsection{Multiple Integrals}

Definition 6.17. Let $\tau: M \times S^{1} \times S^{1} \rightarrow M \times S^{1} \times S^{1},(m, z, w) \mapsto(m, w, z)$ denote the flip. We define integration 'over the first variable' by

$$
\int^{\prime}: \hat{E}^{n+1}\left(M \times S^{1} \times S^{1}\right) \stackrel{\tau}{\rightarrow} \hat{E}^{n+1}\left(M \times S^{1} \times S^{1}\right) \stackrel{\int}{\rightarrow} \hat{E}^{n}\left(M \times S^{1}\right) .
$$

Proposition 6.18. We have $\iint^{\prime}=-\iint: \hat{E}^{n+2}\left(M \times S^{1} \times S^{1}\right) \rightarrow \hat{E}^{n}(M)$.

The proof of this proposition is rather cumbersome due to the indirect definition of (68) and will occupy the remainder of this section.

The first step is the reduction to the integral for pairs using the following lemma. We will use the maps

$$
\begin{aligned}
& i_{1}: M \times S^{1} \times 1 \longleftrightarrow M \times S^{1} \times S^{1}: \mathrm{pr}_{1}, \\
& i_{2}: M \times 1 \times S^{1} \longleftrightarrow M \times S^{1} \times S^{1}: \mathrm{pr}_{2},
\end{aligned}
$$


and their relative versions

$$
\begin{aligned}
& i_{1}^{\prime}:\left(M \times S^{1} \times 1, M \times 1 \times 1\right) \longleftrightarrow\left(M \times S^{1} \times S^{1}, M \times 1 \times S^{1}\right): \mathrm{pr}_{1}^{\prime}, \\
& i_{2}^{\prime}:\left(M \times 1 \times S^{1}, M \times 1 \times 1\right) \longleftrightarrow\left(M \times S^{1} \times S^{1}, M \times S^{1} \times 1\right): \mathrm{pr}_{2}^{\prime} .
\end{aligned}
$$

Lemma 6.19. Let $\hat{x} \in \hat{E}^{n+2}\left(M \times S^{1} \times S^{1}, M \times 1 \times S^{1}\right)$. Then

$$
\hat{y}=\hat{x}-\operatorname{prr}_{1}^{* *} i_{1}^{*} \hat{x}
$$

may be represented by a triple $(c, \omega, h)$ with

$$
c \mid M \times\left(S^{1} \vee S^{1}\right)=\text { const, } i_{1}^{*} \omega=i_{2}^{*} \omega=0, \quad h \mid M \times\left(S^{1} \vee S^{1}\right)=0 .
$$

Proof. Replacing $\hat{x}=[c, \omega, h]$ by $\hat{x}-\operatorname{pr}_{1}^{\prime *} i_{1}^{* *} \hat{x}$ leaves $(70)$ unchanged. We may therefore assume that $i_{1}^{\prime *} \hat{x}=0$. This means that $i_{1}^{*} \omega=0$ and that we have a homotopy

$$
C_{1}: c \circ i_{1}^{\prime} \simeq \text { const } \quad(\text { rel } M \times 1 \times 1) .
$$

By extending this homotopy with $c$ and the constant map, we get a map $[0,1] \times M \times S^{1} \times 1 \cup[0,1] \times M \times 1 \times S^{1} \cup 0 \times M \times S^{1} \times S^{1} \stackrel{C_{1} \cup \text { const } \cup c}{\longrightarrow} E_{n+2}$. Because $M \times\left(S^{1} \vee S^{1}\right) \subset M \times S^{1} \times S^{1}$ is a closed cofibration, this map may be extended to a homotopy $C\left(\right.$ rel $\left.M \times 1 \times S^{1}\right)$ from $c$ to some map $c^{\prime}$ with $c^{\prime} \mid M \times\left(S^{1} \vee S^{1}\right)=$ const. From Proposition 3.13 it follows that

$$
\hat{x}=\left[c^{\prime}, \omega, h^{\prime}\right] \text { for } h^{\prime}=h-\int C^{*} \iota_{n} .
$$

Now compute, using that $N_{n}$ is reduced and Remark 5.24

$$
\begin{aligned}
-\operatorname{pr}_{1}^{\prime *} i_{1}^{\prime *} \hat{x} & =\left[\nu_{n} \circ c^{\prime} \circ i_{1}^{\prime} \circ \operatorname{pr}_{1}^{\prime}, 0,-\operatorname{pr}_{1}^{\prime *} i_{1}^{\prime *} h^{\prime}+\left(c^{\prime} i_{1}^{\prime} \operatorname{pr}_{1}^{\prime}\right)^{*} N_{n}\right] \\
& =\left[\text { const }, 0,-\operatorname{pr}_{1}^{\prime *} i_{1}^{\prime *} h^{\prime}\right] \\
\hat{x}-\operatorname{pr}_{1}^{\prime} i_{1}^{\prime} \hat{x} & =\left[\alpha_{n}\left(c^{\prime}, \text { const }\right), \omega, h-\operatorname{pr}_{1}^{\prime *} i_{1}^{* *} h^{\prime}+\left(c^{\prime}, \text { const }\right)^{*} A_{n}\right] .
\end{aligned}
$$

This is the required triple representative ( $\alpha_{n}$ is pointed and $A_{n}$ reduced).

Remark 6.20. The statement $\iint^{\prime}=-\iint$ is true for cochains (by the symmetry properties of the Eilenberg-Zilber map), differential forms (since $d t \wedge d s=-d s \wedge d t$ ), and for $E$-cocycles in the sense that

$$
\iint \tau^{*} c=t \circ \iint c, \quad \text { for } \quad c: M \times S^{1} \times S^{1} \rightarrow E_{n+2},
$$

for the twist $t: E_{n} \rightarrow \Omega^{2} E_{n+2} \stackrel{\text { flip }}{\longrightarrow} \Omega^{2} E_{n+2} \leftarrow E_{n}$. 
In addition to the maps introduced above, let

$$
\begin{aligned}
& j_{1}: M \times S^{1} \times S^{1} \rightarrow\left(M \times S^{1} \times S^{1}, M \times S^{1} \times 1\right), \\
& j_{2}: M \times S^{1} \times S^{1} \rightarrow\left(M \times S^{1} \times S^{1}, M \times 1 \times S^{1}\right),
\end{aligned}
$$

and

$$
i: M \times 1 \longleftrightarrow M \times S^{1}: \mathrm{pr}, \quad j: M \times S^{1} \rightarrow\left(M \times S^{1}, M \times 1\right) .
$$

Proof of Proposition 6.18. Let $\hat{z} \in \hat{E}^{n+2}\left(M \times S^{1} \times S^{1}\right)$ and choose $\hat{x} \in$ $\hat{E}^{n+2}\left(M \times S^{1} \times S^{1}, M \times 1 \times S^{1}\right)$ with $j_{2}^{*} \hat{x}=\hat{z}-\operatorname{pr}_{2}^{*} i_{2}^{*} \hat{z}$ (possible by 3.15). Then $\hat{y}:=\hat{x}-\operatorname{pr}_{1}^{\prime *} i_{1}^{\prime *} \hat{x}$ may be represented by a triple $(c, \omega, h)$ as in Lemma 6.19 . Using the formal rules of Theorem 6.15 one calculates

$$
\iint-\hat{z}=\iint-\hat{y}, \quad \iint \tau^{*} \hat{z}=\iint j_{1}^{*} \tau^{*} \hat{y}
$$

It suffices therefore to prove the analog of Proposition 6.18 for an element $\hat{y}=[c, \omega, h]$ which may be written as in Lemma 6.19.

In this case, the triple $\left(c \circ \tau, \tau^{*} \omega, \tau^{*} h\right)$ may be viewed as a representative in $\hat{E}^{n+2}\left(M \times S^{1} \times S^{1}, M \times S^{1} \times 1\right)$ as in the definition 69 of the absolute integral of $j_{1}^{*} \tau^{*} \hat{y}$, so by Definition 6.11.

$$
\int j_{1}^{*} \tau^{*} \hat{y}=\left[\int c \circ \tau, \int \tau^{*} \omega, \int \tau^{*} h\right] \in \hat{E}^{n+1}\left(M \times S^{1}\right) .
$$

The triple on the right represents an element of $\hat{E}^{n+1}\left(M \times S^{1}, M \times 1\right)$ as in the definition (69) of the absolute integral of $\int j_{1}^{*} \tau^{*} \hat{y}$, so

$$
\iint j_{1}^{*} \tau^{*} \hat{y}=\left[\iint c \circ \tau, \iint \tau^{*} \omega, \iint \tau^{*} h\right] .
$$

Similarly, we deduce the first equality in

$$
\iint-\hat{y}=-\left[\iint c, \iint \omega, \iint h\right]=\left[\nu_{n} \iint c,-\iint \omega,-\iint h+\left(\iint c\right)^{*} N_{n}\right] .
$$

Using Remark 6.20 and (54) we now compute

$$
\iint-\hat{y}+\iint j_{1}^{*} \tau^{*} \hat{y}=\left[\alpha_{n}\left(\nu_{n} \iint c, \iint c \tau\right), 0,\left(\iint c\right)^{*} N_{n}+\left(\nu_{n} \iint c, \iint c \tau\right)^{*} A_{n}\right] .
$$

We wish to show that this element is zero. Choose the homotopy $h: \operatorname{id} \oplus \nu_{n} \rightarrow$ const defined from Example 5.22. According to Lemma 3.10 and Proposition 3.13 it suffices to show that $\left(\iint c\right)^{*} N_{n}+\left(\nu_{n} \iint c, \iint c \tau\right)^{*} A_{n}-\operatorname{ch}\left(\left(\iint c\right)^{*} h\right)$ is a coboundary. But this assertion is just the pullback along $\iint c$ of (53). 


\section{ACYClic Models Theorem}

The product of differential characters is defined in [CS85] by using an explicit chain homotopy which relates the exterior product of differential forms and the cup product of the corresponding cochains. Such homotopies may also be obtained abstractly from the theory of acyclic models. This approach is particularly useful because it makes the relationship between higher combinations of these homotopies clear.

The goal of the first two sections is to establish a rather general Acyclic Models Theorem for functors that are corepresentable on the models which, in this form, seems not to be in the literature. Our treatment is an adaption of standard ideas [Bar02], Dol95].

\subsection{Cohomological Version}

\subsubsection{Corepresentability on Models}

Definition 7.1. The pair $(\mathscr{C}, \mathcal{M})$ of a category and a full subcategory $\mathcal{M}$ is called a category with models. In this case we have a functor ' ' on the presheaf categories

$$
\operatorname{Fun}\left(\mathscr{C}^{\mathrm{op}}, \operatorname{Mod}_{R}\right) \longrightarrow \operatorname{Fun}\left(\mathscr{C}^{\mathrm{op}}, \operatorname{Mod}_{R}\right)
$$

defined on objects $F: \mathscr{C}^{\mathrm{op}} \rightarrow \operatorname{Mod}_{R}$ by

$$
\begin{aligned}
& \widehat{F} X=\prod_{\substack{\sigma: M \rightarrow X \\
M \in \mathcal{M}}} F M \times\{\sigma\}=\left\{\left(x_{\sigma}\right)_{\sigma: M \rightarrow X, M \in \mathcal{M}} \mid x_{\sigma} \in F M\right\}, \\
& \widehat{F}(X \stackrel{\varphi}{\rightarrow} Y): \widehat{F} Y \rightarrow \widehat{F} X, \quad\left\{y_{\tau}\right\}_{\tau: M \rightarrow Y} \mapsto\left\{y_{\varphi \sigma}\right\}_{\sigma: M \rightarrow X} .
\end{aligned}
$$

To a morphism $\Theta: F \rightarrow G$ the functor " ${ }^{\wedge}$, assigns

$$
\widehat{\Theta}_{X}: \widehat{F} X \rightarrow \widehat{G} X,\left\{x_{\sigma}\right\}_{\sigma: M \rightarrow X} \mapsto\left\{\Theta_{M}\left(x_{\sigma}\right)\right\}_{\sigma: M \rightarrow X} .
$$

Observe that this last formula makes sense for any family $\left(\Theta_{M}\right)_{M \in \mathcal{M}}$ of morphisms $F M \rightarrow G M$ on the models.

There is a canonical natural transformation $\Phi: \operatorname{id}_{\mathbf{F u n}\left(\mathscr{C} \circ \mathrm{P}, \operatorname{Mod}_{R}\right)} \Rightarrow^{\widehat{ }}$ from the identity functor to ${ }^{\text {‘) }}$ defined as

$$
\Phi(F): F \rightarrow \widehat{F}, \quad \Phi(F)_{X}: F X \rightarrow \widehat{F} X, x \mapsto\{F(\sigma) x\}_{\sigma: M \rightarrow X}
$$


Definition 7.2. A functor $F: \mathscr{C}^{\mathrm{op}} \rightarrow \operatorname{Mod}_{R}$ is corepresentable with respect to the models $\mathcal{M}$ if there exists a natural transformation

$$
\Psi: \widehat{F} \rightarrow F
$$

with the property that $\Psi \circ \Phi(F)=\mathrm{id}_{F}$.

Remark 7.3. There is a similarity between the definition of " $\wedge$, and the (dual of the) way one establishes a class of generating cofibrations for the projective model structure on diagram categories [Lur09, p.824]. It seems plausible that there exists a model structure on $\mathbf{F u n}\left(\mathscr{C}, \mathbf{C o C h}_{\mathbb{R}}^{\geq 0}\right)$ in which the weak equivalences are the quasi-isomorphisms on the models and the corepresentable functors are (retracts of generating) fibrations. This would make the Acyclic Models Theorem below a simple lifting argument (the chain contractible functors below would admit a trivial cofibration from the initial object) and therefore yield a well-defined filler up to contractible choice. The only difficulty with this approach is that the notion of 'class of generating fibrations' is not a very useful one (as opposed to cofibrations) - only few 'naturally' appearing model categories admit them.

Example 7.4. Let $\mathscr{C}=$ Man and $\mathcal{M}=\left\{\Delta^{n} \mid n \geq 0\right\}$. Then the smooth $k$-cochain functor $C_{s}^{k}(-; A)$ is corepresentable with respect to $\mathcal{M}$ :

$$
\Psi_{X}: \widehat{C}_{s}^{k}(X ; A) \rightarrow C_{s}^{k}(X ; A),\left\{x_{\sigma}\right\}_{\sigma: \Delta^{n} \rightarrow X} \mapsto\left(C_{k}^{s}(X) \rightarrow A, \sigma \mapsto x_{\sigma}\left(\operatorname{id}_{\Delta^{k}}\right)\right)
$$

Example 7.5. Let $\mathscr{C}=\operatorname{Man} \times$ Man and $\mathcal{M}=\left\{\left(\Delta^{n}, \Delta^{m}\right) \mid n, m \geq 0\right\}$. Then $C_{s}^{k}(M \times N ; A)$ is corepresentable with respect to $\mathcal{M}$ by choosing

$$
\begin{aligned}
\Psi_{X, Y}\left\{x_{\sigma, \tau}\right\}_{\sigma: \Delta^{n} \rightarrow X}: & C_{k}^{s}(X \times Y ; A) \longrightarrow A, \\
\tau: \Delta^{m} \rightarrow Y & \longrightarrow \\
& \left(\Delta^{k} \stackrel{(\sigma, \tau)}{\longrightarrow} X \times Y\right) \longmapsto x_{\sigma, \tau}\left(\Delta^{k} \stackrel{\text { diag }}{\longrightarrow} \Delta^{k} \times \Delta^{k}\right) .
\end{aligned}
$$

\subsubsection{Chain Contractibility}

Definition 7.6. A functor $F: \mathscr{C}^{\mathrm{op}} \rightarrow \mathbf{C o C h}_{R}^{\geq 0}$ is chain contractible on the models $M \in \mathcal{M}$ in degree $n$ if there exist homomorphisms of $R$-modules

$$
h_{M}^{n}: F^{n}(M) \rightarrow F^{n-1}(M), \quad h_{M}^{n+1}: F^{n+1}(M) \rightarrow F^{n}(M) \quad(\forall M \in \mathcal{M})
$$


with the property that

$$
\operatorname{id}_{F^{n}(M)}=h_{M}^{n+1} \circ \delta+\delta \circ h_{M}^{n}
$$

Example 7.7. For $R=\mathbb{R}$ this just means $H^{n}(F(M))=0$.

Proof. Clearly, chain contractibility in degree $n$ implies $H^{n}(F(M))=0$. Conversely, let $C^{*}=F^{*}(M)$, so that

$$
\cdots \longrightarrow C^{n-1} \stackrel{\delta^{n-1}}{\longrightarrow} C^{n} \stackrel{\delta^{n}}{\longrightarrow} C^{n+1} \longrightarrow \cdots
$$

is exact at $C^{n}$. Since $\delta: C^{n} \rightarrow \delta\left(C^{n}\right)$ is surjective there is a section $h^{n+1}$ : $\delta\left(C^{n}\right) \rightarrow C^{n}$ with $\delta \circ h^{n+1}=\operatorname{id}_{\delta\left(C^{n}\right)}$. We extend $h^{n+1}$ to $C^{n+1}$ by chosing a complementary subspace of $\delta\left(C^{n}\right)$ and by defining $h^{n+1}$ to be zero there. Let $\left(e_{i}\right)_{i \in I}$ denote a basis of $C^{n}$. Then

$$
e_{i}-h^{n+1} \delta^{n}\left(e_{i}\right) \in \operatorname{ker}\left(\delta^{n}\right)=\operatorname{im}\left(\delta^{n-1}\right)
$$

so that we may write $e_{i}-h^{n+1} \delta^{n}\left(e_{i}\right)=\delta^{n-1}\left(f_{i}\right)$ for some $f_{i} \in C^{n-1}$. Now define $h^{n}$ by letting $h^{n}\left(e_{i}\right)=f_{i}, i \in I$.

\subsubsection{Acyclic Models Theorem}

Lemma 7.8. Given a commutative diagram of functors $\mathscr{C}^{\mathrm{op}} \rightarrow \operatorname{Mod}_{R}$ and natural transformations

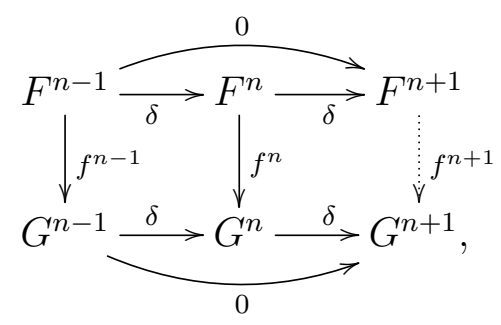

there exists the indicated natural extension $f^{n+1}$ in case

1. $F^{n}$ is chain contractible on the models,

2. $G^{n+1}$ is corepresentable with respect to $\mathcal{M}$. 
Proof. Choose contracting homotopies $h_{M}^{n}, h_{M}^{n+1}$ for the models. We first define a map $E_{M}^{n+1}$ for each model $M \in \mathcal{M}$ by the composition

$$
E_{M}^{n+1}: F^{n+1}(M) \stackrel{h_{M}^{n+1}}{\rightarrow} F^{n}(M) \stackrel{f^{n}}{\rightarrow} G^{n}(M) \stackrel{\delta}{\rightarrow} G^{n+1}(M) .
$$

Then

$$
E_{M}^{n+1} \delta=\delta f^{n} h_{M}^{n+1} \delta=\delta f^{n}\left(\mathrm{id}-\delta h_{M}^{n}\right)=\delta f^{n}-\delta^{2} f^{n-1} h_{M}^{n}=\delta f^{n}
$$

The required map $f^{n+1}$ is then given by the composition

$$
F^{n+1} \stackrel{\Phi\left(F^{n+1}\right)}{\longrightarrow} \widehat{F}^{n+1} \stackrel{\widehat{E}^{n+1}}{\longrightarrow} \hat{G}^{n+1} \stackrel{\Psi}{\rightarrow} G^{n+1} .
$$

The formula $f^{n+1} \delta=\delta f^{n}$ follows from the naturality of $\Psi$ :

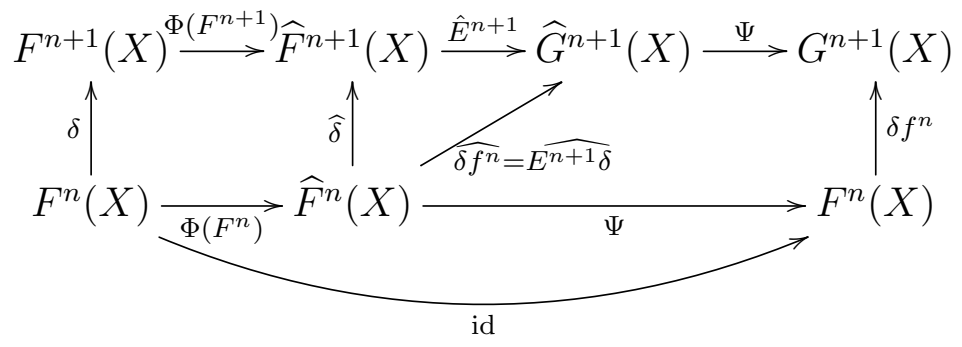

Let us concentrate now on the case $R=\mathbb{R}$ of real vector spaces. The following is a cohomological version of the Acyclic Models Theorem:

Theorem 7.9. Let $F, G: \mathscr{C}^{\mathrm{op}} \rightarrow \mathbf{C o C h}_{\mathbb{R}}^{\geq 0}$ be functors such that $G^{*}$ is corepresentable with respect to $\mathcal{M}$ and $H^{*+1}(F(M))=0(\forall M \in \mathcal{M})$ for all $* \geq 0$. Then any natural transformation $H^{0}(F) \rightarrow H^{0}(G)$ is induced by a natural chain map $F^{*} \rightarrow G^{*}$, unique up to natural chain homotopy.

Proof. The first part is an inductive application of Lemma 7.8 to

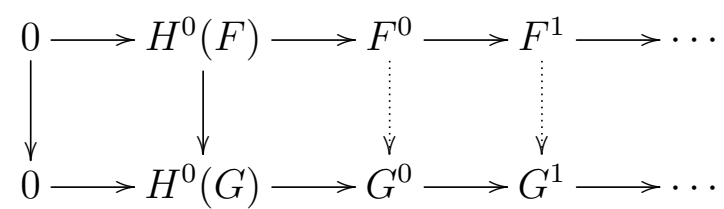


Note here that the first line is exact, so that by Example 7.7 the required chain contractions are available. For the uniqueness statement we have to exhibit a chain homotopy $s^{n}: F^{n} \rightarrow G^{n-1}$ with

$$
f^{n}-g^{n}=\delta s^{n}+s^{n+1} \delta
$$

Set $s^{0}=0$. By Lemma 7.8 we may define $s^{1}$ as a filler in

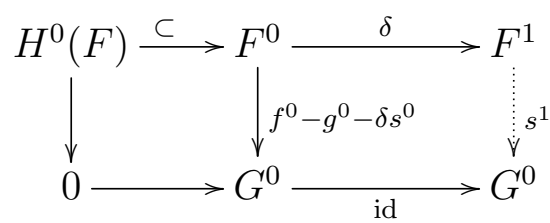

Here the left rectangle commutes since $f^{0}$ and $g^{0}$ are equal on 0 -cocycles, by assumption. For $n=1,2,3, \ldots$ inductively define $s^{n+1}$ as a filler in

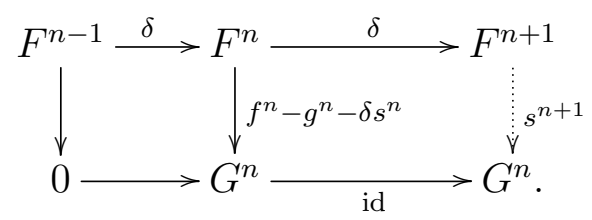

Here, the commutativity of the left rectangle follows by induction:

$$
\left(f^{n}-g^{n}-\delta s^{n}\right) \delta=\delta f^{n-1}-\delta g^{n-1}-\delta\left(f^{n-1}-g^{n-1}-\delta s^{n-1}\right)=0 .
$$

Theorem 7.10. Let $F, G: \mathscr{C}^{\mathrm{op}} \rightarrow \mathbf{C o C h}_{\mathbb{R}}^{\geq 0}$ be functors such that $G^{*}$ is corepresentable with respect to $\mathcal{M}$ and $H^{*+1}(F(M))=0(\forall M \in \mathcal{M})$ for all $* \geq 0$. Suppose given two natural chain maps $f^{*}, g^{*}: F^{*} \rightarrow G^{*}$. Then any two natural chain homotopies $s^{*}, r^{*}: F^{*} \rightarrow G^{*-1}$ are naturally chain homotopic: there exist $t^{*}: F^{*} \rightarrow G^{*-2}$ with

$$
s^{n}-r^{n}=\delta t^{n}-t^{n+1} \delta
$$

Proof. Set $t^{0}=0, t^{1}=0$. Since from $G^{-1}=0$ necessarily $s^{0}, r^{0}=0$, equation (71) is valid for $n=0$. Consider inductively for $n=1,2,3, \ldots$ the diagram

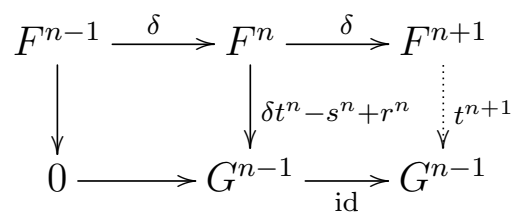


to obtain the required filler $t^{n+1}$. Here, the left rectangle commutes by the inductive assumption:

$$
\begin{aligned}
\left(\delta t^{n}-s^{n}+r^{n}\right) \delta & =\delta t^{n} \delta-s^{n} \delta+r^{n} \delta \\
& =\delta t^{n} \delta-\left(f^{n-1}-g^{n-1}-\delta s^{n-1}\right)+\left(f^{n-1}-g^{n-1}-\delta r^{n-1}\right) \\
& =\delta\left(t^{n} \delta+s^{n-1}-r^{n-1}\right)=\delta \delta t^{n-1}=0
\end{aligned}
$$

\subsection{Applications}

\subsubsection{Coefficients in a Vector Space}

We will use differential forms with coefficients in a real vector space $W$, as defined in [BS10, p.3]. Recall that the cross-product of cochains $u \in$ $C^{n}(X ; V), v \in C^{m}(Y ; W)$ is defined using the Alexander-Whitney map (Definition 5.1) for $A=\mathbb{Z} \operatorname{sing}(X), B=\mathbb{Z} \operatorname{sing}(Y)$ :

$u \times v: C_{n+m}(X \times Y) \stackrel{A W}{\longrightarrow} \bigoplus_{i+j=n+m} C_{i}(X) \otimes C_{j}(Y) \stackrel{\mathrm{pr}}{\longrightarrow} C_{n}(X) \otimes C_{m}(Y) \stackrel{u \otimes v}{\longrightarrow} V \otimes W$.

From properties of the Alexander-Whitney map, it is associative and unital already on the level of cochains. Working instead with smooth singular complexes, we obtain a corresponding definition for smooth cochains.

Example 7.11. Write $\omega_{1} \pi \omega_{2}=\operatorname{pr}_{1}^{*} \omega_{1} \wedge \operatorname{pr}_{2}^{*} \omega_{2}$ for the associated external product of differential forms. The functor

$$
\operatorname{Man}^{\mathrm{op}} \times \operatorname{Man}^{\mathrm{op}} \longmapsto \mathbf{C o C h}_{\mathbb{R}}^{\geq 0},(M, N) \longmapsto \Omega(M ; V) \otimes \Omega(N ; W)
$$

is acyclic on the models $\left(\Delta^{n}, \Delta^{m}\right)$. Consider the natural chain maps

$$
\begin{aligned}
&(\Omega(M ; V) \otimes \Omega(N ; W))^{*} \longrightarrow C_{s}^{*}(M \times N ; V \otimes W), \omega_{0} \otimes \omega_{1} \longmapsto \omega_{0} \pi \omega_{1}, \\
& \omega_{0} \otimes \omega_{1} \longmapsto \omega_{0} \times \omega_{1} .
\end{aligned}
$$

Both these chain maps coincide in degree 0. By Example 7.5, the functor $C_{s}^{*}(M \times N ; V \otimes W)$ is corepresentable on the models. Using Theorem 7.9 we obtain a natural chain homotopy

$$
B:(\Omega(M ; V) \otimes \Omega(N ; W))^{*} \longrightarrow C_{s}^{*-1}(M \times N ; V \otimes W)
$$


satisfying

$$
\delta B\left(\omega_{0} \otimes \omega_{1}\right)+B d\left(\omega_{0} \otimes \omega_{1}\right)=\omega_{0} \pi \omega_{1}-\omega_{0} \times \omega_{1} .
$$

Setting $M=N$ and post-composing with 'pullback along the diagonal,' we obtain a corresponding chain homotopy between the internal products $\wedge, \cup$.

Example 7.12. Next consider the two natural chain maps

$$
\begin{gathered}
(\Omega(L ; U) \otimes \Omega(M ; V) \otimes \Omega(N ; W))^{*} \longrightarrow C_{s}^{*}(L \times M \times N ; U \otimes V \otimes W), \\
\omega_{0} \otimes \omega_{1} \otimes \omega_{2} \longmapsto \omega_{0} \bar{\wedge} \omega_{1} \bar{\wedge} \omega_{2}, \\
\omega_{0} \otimes \omega_{1} \otimes \omega_{2} \longmapsto \omega_{0} \times \omega_{1} \times \omega_{2} .
\end{gathered}
$$

Using $(72)$ we see that

$$
\begin{aligned}
& \omega_{0} \otimes \omega_{1} \otimes \omega_{2} \mapsto B\left(\left(\omega_{0} \bar{\wedge} \omega_{1}\right) \otimes \omega_{2}\right)+B\left(\omega_{0} \otimes \omega_{1}\right) \times \omega_{2}, \\
& B\left(\omega_{0} \otimes\left(\omega_{1} \pi \omega_{2}\right)\right)+(-1)^{\left|\omega_{0}\right|} \omega_{0} \times B\left(\omega_{1} \otimes \omega_{2}\right),
\end{aligned}
$$

define two natural chain homotopies between these two chain maps. By Theorem 7.10 these are themselves chain homotopic.

Example 7.13. We have two natural chain maps

$$
\begin{aligned}
C_{s}(M ; V) \otimes C_{s}(N ; W) & \longrightarrow C_{s}(M \times N ; V \otimes W), \\
u \otimes v & \longmapsto u \times v, \\
u \otimes v & \longmapsto(-1)^{|u||v|} \operatorname{flip}^{*}(v \times u),
\end{aligned}
$$

which induce the same map in degree zero (flip* includes the flip of coefficients). Theorem 7.9 yields a natural chain homotopy

$$
D:\left(C_{s}(M ; V) \otimes C_{s}(N ; W)\right)^{*} \longrightarrow C_{s}^{*-1}(M \times N ; V \otimes W)
$$

between these maps:

$$
\delta D(u \otimes v)+D \delta(u \otimes v)=u \times v-(-1)^{|u||v|} \operatorname{flip}^{*}(v \times u) .
$$

Example 7.14. The exterior product of differential forms is graded commutative: $\omega_{0} \bar{\wedge} \omega_{1}=(-1)^{\left|\omega_{0}\right|\left|\omega_{1}\right|}$ flip $^{*}\left(\omega_{1} \bar{\wedge} \omega_{0}\right)$. It follows that between the two 
natural chain maps

$$
\begin{aligned}
&(\Omega(M ; V) \otimes \Omega(N ; W))^{*} \longrightarrow C_{s}^{*}(N \times M ; V \otimes W) \\
& \omega_{0} \otimes \omega_{1} \longmapsto(-1)^{\left|\omega_{0}\right|\left|\omega_{1}\right|} \omega_{1} \pi \omega_{0}, \\
& \omega_{0} \otimes \omega_{1} \longmapsto(-1)^{\left|\omega_{0}\right|\left|\omega_{1}\right|} \omega_{1} \times \omega_{0},
\end{aligned}
$$

the following associations define two natural chain homotopies

$$
\begin{aligned}
& \omega_{0} \otimes \omega_{1} \longmapsto(-1)^{\left|\omega_{0}\right|\left|\omega_{1}\right|} B\left(\omega_{1} \otimes \omega_{0}\right), \\
& \omega_{0} \otimes \omega_{1} \longmapsto \operatorname{fip}^{*} B\left(\omega_{0} \otimes \omega_{1}\right)-(-1)^{\left|\omega_{0}\right|\left|\omega_{1}\right|} D\left(\omega_{1} \otimes \omega_{0}\right) .
\end{aligned}
$$

By Theorem 7.10, these are themselves chain-homotopic. In particular, for closed forms $\omega_{0}, \omega_{1}$ we have, up to coboundaries,

$$
(-1)^{\left|\omega_{0}\right|\left|\omega_{1}\right|} B\left(\omega_{0} \otimes \omega_{1}\right) \equiv \operatorname{flip}^{*} B\left(\omega_{1} \otimes \omega_{0}\right)-(-1)^{\left|\omega_{0}\right|\left|\omega_{0}\right|} D\left(\omega_{0} \otimes \omega_{1}\right) .
$$

Example 7.15. As another application, consider the functor

$$
F^{n}(M)= \begin{cases}\left(\Omega(M ; V) \otimes \Omega\left(N \times S^{1} \times S^{1} ; W\right)\right)^{n+2} & (n \geq 0), \\ 0 & (n<0),\end{cases}
$$

and the two natural chain maps given in positive degrees $* \geq 0$ by

$$
\begin{aligned}
& F^{*}(M) \longrightarrow C_{s}^{*}(M \times N ; V \otimes W), \omega_{0} \otimes \omega_{1} \longmapsto \omega_{0} \times\left(\int_{S^{1}} \int_{S^{1}} \omega_{1}\right), \\
& \omega_{0} \otimes \omega_{1} \longmapsto \int_{S^{1}} \int_{S^{1}}\left(\omega_{0} \pi \omega_{1}\right),
\end{aligned}
$$

and otherwise by zero. It follows from Theorem 7.10 that the two natural chain homotopies

$$
\begin{aligned}
& \int_{S^{1}} \int_{S^{1}} B\left(\omega_{0} \otimes \omega_{1}\right), \\
& B\left(\omega_{0} \otimes\left(\int_{S^{1}} \int_{S^{1}} \omega_{1}\right)\right),
\end{aligned}
$$

between these two natural chain maps are themselves chain homotopic. 


\subsubsection{Generalization to Graded Coefficients}

We begin by discussing the cross-product on $C^{n}(X ; V)$ in case $V^{*}$ is a graded commutative real algebra. Let $u \in C^{n}(X ; V), v \in C^{m}(Y ; V)$, corresponding to left bounded sequences

$$
\begin{gathered}
u^{0} \in C^{0}\left(X ; V^{n}\right), u^{1} \in C^{1}\left(X ; V^{n-1}\right), \ldots, u^{i} \in C^{i}\left(X ; V^{n-i}\right), \ldots, \\
v^{0} \in C^{0}\left(Y ; V^{m}\right), v^{1} \in C^{1}\left(Y ; V^{m-1}\right), \ldots, v^{j} \in C^{j}\left(Y ; V^{m-j}\right), \ldots
\end{gathered}
$$

To define $w=u \times v \in C^{n+m}(X \times Y ; V)$ we need to specify elements $w^{k} \in$ $C^{k}\left(X \times Y ; V^{n+m-k}\right)$ for each $k \geq 0$ :

$$
w^{k}=\sum_{i+j=k}(-1)^{(n-i) j} u^{i} \times v^{j}
$$

Every such sum is finite since $i, j \geq 0$ (we have started with positive cochain complexes). In (75) we have used the multiplication on $V$ :

$C^{i}\left(X ; V^{n-i}\right) \otimes C^{j}\left(Y ; V^{m-j}\right) \rightarrow C^{i+j}\left(X \times Y ; V^{n-i} \otimes V^{m-j}\right) \rightarrow C^{i+j}\left(X \times Y ; V^{n+m-i-j}\right)$.

The cross-product restricts to smooth cochains, a fact that descends to the graded case. Replacing ' $X$ ' by ' $\bar{\wedge}$ ' in $(\overline{75})$, the exterior product of differential forms may also be extended to graded coefficients (the sign has been chosen so as to make this extension graded commutative). Similarly for internal products. The differentials are given by $(d \omega)^{k}=d \omega^{k-1}$. With these sign conventions, the usual formulas for forms and cochains are retained, e.g.,

$$
\begin{aligned}
d(\omega \pi \eta) & =d \omega \bar{\wedge} \eta+(-1)^{|\omega|} \omega \bar{\wedge} d \eta, \quad \delta(u \times v)=\delta u \times v+(-1)^{|u|} u \times \delta v, \\
\omega \bar{\wedge} \eta & =(-1)^{|\omega| \cdot|\eta|} \text { flip }^{*}(\eta \bar{\wedge} \omega) .
\end{aligned}
$$

In particular, we have two chain maps

$$
\begin{aligned}
(\Omega(M ; V) \otimes \Omega(N ; V))^{*} \longrightarrow C_{s}^{*}(M \times N ; V), & \omega_{0} \otimes \omega_{1} \longmapsto \omega_{0} \bar{\wedge} \omega_{1}, \\
& \omega_{0} \otimes \omega_{1} \longmapsto \omega_{0} \times \omega_{1} .
\end{aligned}
$$

From Example 7.11 we get maps

$$
\begin{aligned}
B: \Omega^{i}\left(M ; V^{n-i}\right) \otimes \Omega^{j}\left(N ; V^{m-j}\right) & \rightarrow C^{i+j-1}\left(M \times N ; V^{n-i} \otimes V^{m-j}\right) \\
& \rightarrow C^{i+j-1}\left(M \times N ; V^{n+m-i-j}\right)
\end{aligned}
$$


which piece together a natural chain homotopy

$$
B:(\Omega(M ; V) \otimes \Omega(N ; V))^{*} \rightarrow C_{s}^{*-1}(M \times N ; V) .
$$

between these two chain maps. In detail, for $\omega \in \Omega^{n}(M ; V), \eta \in \Omega^{m}(N ; V)$, $B(\omega, \eta)$ is given by the sequence of ordinary cochains

$$
\sum_{i+j=k}(-1)^{(n-i) j} B\left(\omega^{i}, \eta^{j}\right) \in C^{k-1}\left(M \times N ; V^{n+m-k}\right)
$$

It is important to observe that this sum is finite since $i, j \geq 0$. Similarly, the chain homotopies from the other examples generalize to graded coefficients.

\section{Products (Rationally Even Case)}

In this chapter, we will work out the product structure on the differential cohomology groups, starting from a ring spectrum E up to homotopy 7 . This lack of structure will be compensated by assuming $E$ to be rationally even, which means that

$$
\pi_{2 n+1}(E) \otimes \mathbb{R}=0, \quad \forall n \in \mathbb{Z} .
$$

For the construction of products in differential cohomology we will need to choose the fundamental cocycles $\iota_{n}$ coherently compatible with the cup product. This means that $\iota_{n} \times \iota_{m}$ and $\mu_{n, m}^{*} \iota_{n+m}$ should differ in a controlled way, where $\mu_{n, m}: E_{n} \wedge E_{m} \rightarrow E_{n+m}$ represents multiplication.

Assumption (77) will imply all the coherence conditions we are after, at least in even degrees. It will then be our strategy to 'transport' this structure to odd degrees by demanding compatibility with the integration map.

\subsection{Construction in Even Degrees}

The goal of this section is the construction of a canonical refinement of $\hat{E}^{2 *}$ to a functor into unital (graded) commutative rings in a such a way that

$$
a(\theta) \cup \hat{x}=a(\theta \wedge R \hat{x}), \quad \forall \theta \in \Omega^{n-1}(M ; V), \hat{x} \in \hat{E}^{m}(M) .
$$

In the next section, we will extend the product to odd degrees by using the integration map. For this reason, it is slightly more convenient to work with external products.

\footnotetext{
${ }^{7}$ In fact, we will just need a multiplicative cohomology theory.
} 


\subsubsection{Internal and External Products}

For clarity, we record the following standard observation:

Proposition 8.1. Given functors $E^{*}: \mathbf{M a n}^{\mathrm{op}} \rightarrow \mathbf{A b}, * \in \mathbb{Z}$ (or any submonoid of $(\mathbb{Z},+, 0))$. Then the following structures correspond bijectively to each other:

1. Natural External Products, i.e., natural transformations

$$
\times: E^{n}(N) \otimes E^{m}(M) \rightarrow E^{n+m}(N \times M), \quad n, m \in \mathbb{Z} .
$$

2. Natural Internal Products, i.e., natural transformations

$$
\cup: E^{n}(M) \otimes E^{m}(M) \rightarrow E^{n+m}(M), \quad n, m \in \mathbb{Z}
$$

This correspondence is given by ( $\Delta$ denotes the diagonal)

$$
x \cup y=\Delta^{*}(x \times y), \quad x \times y=\operatorname{pr}_{1}^{*} x \cup \operatorname{pr}_{2}^{*} y .
$$

The notions of associativity and commutativity correspond to each other:

$$
\begin{array}{rlrl}
(u \cup v) \cup w & =u \cup(v \cup w) & (u \times v) \times w & =u \times(v \times w) \\
u \cup v & =(-1)^{|u||v|} v \cup u & u \times v & =(-1)^{|u||v|} \operatorname{flip}^{*}(v \times u) .
\end{array}
$$

Moreover, in this situation, the following structures are equivalent:

1. An element $1_{\mathrm{pt}} \in E^{0}(\mathrm{pt})$ which acts as a unit: $u \times 1=u, 1 \times u=u$ (using the identifications $M \times \mathrm{pt}=M$, pt $\times M=M$ ).

2. Elements $1_{M} \in E^{0}(M)$ for each $M \in$ Man which are units in $E^{*}(M)$ and which are preserved by each induced homomorphism $E^{0}(f)$.

\subsubsection{Setup}

Let $E$ be a multiplicative cohomology. By the Brown Representability Theorem 2.3. we may choose pointed maps

$$
\mu_{n, m}: E_{n} \wedge E_{m} \rightarrow E_{n+m}, \quad u: S^{0} \rightarrow E_{0},
$$


representing multiplication and the unit. They are unique up to homotopy. Therefore, the usual diagrams expressing the axioms of associativity, commutativity, etc., commute in the homotopy category of spaces. We will view $u$ as a map

$$
u: \mathrm{pt} \rightarrow E_{0}
$$

According to Corollary 2.9, there is a canonical natural transformation of multiplicative cohomology theories

$$
\operatorname{ch}: \tilde{E}^{n}(X)=\left[X, E_{n}\right] \rightarrow \tilde{H}^{n}(X ; V), \quad V=\tilde{E}^{*}\left(S^{0}\right) \otimes \mathbb{R} .
$$

Recall also from (16) the fundamental cocycles

$$
\iota_{n} \in \tilde{Z}^{n}\left(E_{n} ; V\right) \quad \text { with } \quad \iota_{n}=\int \varepsilon_{n}^{*} \iota_{n+1} .
$$

These represent the fundamental cohomology classes $\operatorname{ch}\left(\operatorname{id}_{E_{n}}\right)=\left[\iota_{n}\right] \in \tilde{H}^{n}(E ; V)$ that implement the Chern character $(79)$ by way of Yoneda's Lemma.

Since $\operatorname{ch}\left(\mathrm{id}_{E_{n}} \times \mathrm{id}_{E_{m}}\right)=\operatorname{ch}\left(\mathrm{id}_{E_{n}}\right) \times \operatorname{ch}\left(\mathrm{id}_{E_{m}}\right)$ we may choose reduced cochains

$$
M_{n, m} \in \tilde{C}^{n+m-1}\left(E_{n} \wedge E_{m} ; V\right) \quad \text { with } \quad \delta M_{n, m}=\iota_{n} \times \iota_{m}-\mu_{n, m}^{*} \iota_{n+m}
$$

We make this choice only for $n, m$ even. Similarly, $\omega_{\mathrm{pt}} \in \Omega^{0}(\mathrm{pt} ; V)$ represents the unit $1 \in H^{0}(\mathrm{pt} ; V)$, and unitality of $(79)$ implies the existence of a cochain

$$
U \in C^{-1}(\mathrm{pt} ; V) \quad \text { with } \quad \delta U=\omega_{\mathrm{pt}}-u^{*} \iota_{0}
$$

Lemma 8.2. Suppose $E$ is rationally even 77 . Then, if $V=\tilde{E}^{*}\left(S^{0}\right) \otimes \mathbb{R}$,

$$
\begin{aligned}
\tilde{H}^{*}\left(E_{n} ; V\right) & =0 & & (n \text { even }) \\
\tilde{H}^{*}\left(E_{n} \times E_{m} ; V\right) & =0 & & (n, m \text { even })
\end{aligned}
$$

for all odd degrees $* \in \mathbb{Z}$. Similarly for higher products.

Proof. This follows from [BS10, Lemma 3.8], Universal Coefficients, and the Künneth Theorem.

The following consequence of this lemma plays a central role in this section:

$(\diamond)$ If $x, y \in \tilde{C}^{k}\left(E_{n} \times E_{m} \times \cdots ; V\right)$ are cochains in odd degree $k$ with $\delta x=\delta y$, then $x$ and $y$ differ by a coboundary. 
For example, any two choices of (80) and (81) differ by a coboundary. Recall that for integration on the left (for example, in (19)) Stokes' formula reads

$$
\delta \int_{I}+\int_{I} \delta=i_{1}^{*}-i_{0}^{*}
$$

\subsubsection{Explicit Construction}

For the construction of representatives $(c, \omega, h)$ for the product of differential cohomology classes it is important to use the chain homotopy $B$ from 76 . which relates the cross-product with the exterior product of differential forms. This is because Definition 3.3 requires that

$$
\delta h=\omega-c^{*} \iota_{n} .
$$

Definition 8.3. For $n, m$ even let $x_{0}=\left(c_{0}, \omega_{0}, h_{0}\right)$ and $x_{1}=\left(c_{1}, \omega_{1}, h_{1}\right)$ be representatives of elements of $\hat{E}^{n}(M)$ and $\hat{E}^{m}(N)$. Set

$x_{0} \times x_{1}=\left(\mu_{n m}\left(c_{0} \times c_{1}\right), \omega_{0} \bar{\wedge} \omega_{1}, B\left(\omega_{0} \otimes \omega_{1}\right)+h_{0} \times \omega_{1}+\omega_{0} \times h_{1}-h_{0} \times \delta h_{1}+\left(c_{0} \times c_{1}\right)^{*} M_{n, m}\right)$.

Recall that a differential cohomology class is an equivalence class of differential cocycles $(c, \omega, h)$. Definition 8.3 descends to a well-defined multiplication of equivalence classes:

Lemma 8.4. The differential cohomology class of $x_{0} \times x_{1}$ in $\hat{E}^{n+m}(M \times N)$ is well-defined and depends only upon the equivalence classes of $x_{0}$ and $x_{1}$. It is also independent of the choices (78), (80).

Proof. By choice of $(76)$ and $(80)$ we have

$$
\begin{aligned}
\delta\left(B\left(\omega_{0} \otimes \omega_{1}\right)\right. & \left.+h_{0} \times \omega_{1}+\omega_{0} \times h_{1}-h_{0} \times \delta h_{1}+\left(c_{0} \times c_{1}\right)^{*} M_{n, m}\right) \\
& =\omega_{0} \wedge \omega_{1}-\left(c_{0}, c_{1}\right)^{*} \mu_{n, m}^{*} \iota_{n+m} .
\end{aligned}
$$

Therefore, $x_{0} \times x_{1}$ represents an element in $\hat{E}^{n+m}(M \times N)$. Suppose $\left(C_{0}, H_{0}\right)$, $\left(C_{1}, H_{1}\right)$ witness equivalences $x_{0} \sim\left(c_{0}^{\prime}, \omega_{0}, h_{0}^{\prime}\right)=x_{0}^{\prime}, x_{1} \sim\left(c_{1}^{\prime}, \omega_{1}, h_{1}^{\prime}\right)=x_{1}^{\prime}$. Then the pair of $C=\mu_{n, m}\left(C_{0} \times C_{1}\right)$ and of the cochain

$$
H=B\left(\operatorname{pr}^{*} \omega_{0} \otimes \operatorname{pr}^{*} \omega_{1}\right)+H_{0} \times \operatorname{pr}^{*} \omega_{1}+\operatorname{pr}^{*} \omega_{0} \times H_{1}-H_{0} \times \delta H_{1}+\left(C_{0} \times C_{1}\right)^{*} M_{n, m}
$$


witnesses a corresponding equivalence $x_{0} \times x_{1} \sim x_{0}^{\prime} \times x_{1}^{\prime}$.

Any other choice $\mu_{n, m}^{\prime}$ in (78) leads to homotopic maps $h: \mu_{n, m} \simeq \mu_{n, m}^{\prime}$ for some homotopy $h: I \times\left(E_{n} \wedge E_{m}\right) \rightarrow E_{n+m}$. Choose $M_{n, m}^{\prime}$ for $\mu_{n, m}^{\prime}$ as in (80). Using (18) and (19), we see that in order to prove the last statement of the lemma it suffices to show that the pullback along $c_{0} \times c_{1}$ of

$$
M_{n, m}^{\prime}+\int_{I} h^{*} \iota_{n+m}-M_{n, m}
$$

is a coboundary, which follows from (80), 820 , and $(\diamond)$.

Definition 8.5. Define the unit by

$$
\hat{1}=\left[u, \omega_{\mathrm{pt}}, U\right] \in \hat{E}^{0}(\mathrm{pt}) .
$$

Lemma 8.6. The differential cohomology class of $\hat{1}$ is well-defined and independent of the choices (78), (81).

Proof. The triple represents a differential cocycle by (81). Any other choice of map $u^{\prime}$ in (78) is homotopic to $u$; write $h: u \simeq u^{\prime}$ for the homotopy, and choose $U^{\prime}$ as in (81) for $u^{\prime}$. It suffices to show that $U^{\prime}+\int h^{*} \iota_{0}-U$ is a coboundary, which follows from (81), (82), and $(\diamond)$.

\subsubsection{Verification of Ring Structure in Even Degrees}

Theorem 8.7. Definitions 8.3 and 8.5 determine a bilinear, unital, commutative, and associative external product on even degrees $\hat{E}^{2 *}(M)$.

The proof is rather tedious. We shall omit irrelevant degree indices on $A$, $\iota$, and $M$. In our calculations with differential cocycles we will often combine Leibniz' rule $(\delta u) \times v \equiv u \times(\delta v)$ with $(18)$. We will write $\hat{x}_{i}=\left[c_{i}, \omega_{i}, h_{i}\right]$. The reader should keep in mind that $\delta h_{i}=\omega_{i}-c_{i}^{*} \iota$.

Proof. Bilinear: In view of commutativity below, it is enough to show the equality $\hat{x}_{0} \times\left(\hat{x}_{1}+\hat{x}_{2}\right)=\hat{x}_{0} \times \hat{x}_{1}+\hat{x}_{0} \times \hat{x}_{2}$. Unwinding Definitions 8.3 and 
5.25, we see that we need to compare the class of the triple

$$
\begin{aligned}
c= & \mu\left(c_{0} \times \alpha\left(c_{1}, c_{2}\right)\right), \\
\omega= & \omega_{0} \pi\left(\omega_{1}+\omega_{2}\right), \\
h= & B\left(\omega_{0}, \omega_{1}+\omega_{2}\right)+h_{0} \times\left(\omega_{1}+\omega_{2}\right)+\omega_{0} \times\left(h_{1}+h_{2}+\left(c_{1}, c_{2}\right)^{*} A\right) \\
& -h_{0} \times \delta\left(h_{1}+h_{2}+\left(c_{1}, c_{2}\right)^{*} A\right)+\left(c_{0} \times \alpha\left(c_{1}, c_{2}\right)\right)^{*} M,
\end{aligned}
$$

with the class of

$$
\begin{aligned}
c^{\prime}= & \alpha\left(\mu\left(c_{0} \times c_{1}\right), \mu\left(c_{0} \times c_{2}\right)\right), \\
\omega^{\prime}= & \omega_{0} \pi \omega_{1}+\omega_{0} \pi \omega_{2}, \\
h^{\prime}= & B\left(\omega_{0}, \omega_{1}\right)+B\left(\omega_{0}, \omega_{2}\right)+h_{0} \times \omega_{1}+h_{0} \times \omega_{2}+\omega_{0} \times\left(h_{1}+h_{2}\right) \\
& -h_{0} \times \delta\left(h_{1}+h_{2}\right)+\left(c_{0} \times c_{1}\right)^{*} M+\left(c_{0} \times c_{2}\right)^{*} M+\left(\mu\left(c_{0} \times c_{1}\right), \mu\left(c_{0} \times c_{2}\right)\right)^{*} A .
\end{aligned}
$$

Using bilinearity of $B$ and of ' $x$ ' we see that, up to coboundaries, $h-h^{\prime}$ is

$c_{0}^{*} \iota \times\left(c_{1}, c_{2}\right)^{*} A+\left(c_{0} \times \alpha\left(c_{1}, c_{2}\right)\right)^{*} M-\left(c_{0} \times c_{1}\right)^{*} M-\left(c_{0} \times c_{2}\right)^{*} M-\left(\mu\left(c_{0} \times c_{1}\right), \mu\left(c_{0} \times c_{2}\right)\right)^{*} A$.

Let $\mathrm{pr}_{12}, \mathrm{pr}_{13}$ denote the projections onto the factors 12,13 and let

$$
\pi: E_{n} \times E_{m} \times E_{m} \rightarrow E_{n} \times E_{m} \times E_{n} \times E_{m},(x, y, z) \mapsto(x, y, x, z) .
$$

Select a homotopy $H: \mu(\mathrm{id} \times \alpha) \simeq \alpha(\mu \times \mu) \pi$. To show that the above two triples are equivalent it suffices by (18) and (19) to show that the pullback along $c_{0} \times\left(c_{1}, c_{2}\right)$ of

$\iota_{n} \times A_{m}+\left(\mathrm{id} \times \alpha_{m}\right)^{*} M_{n, m}-\operatorname{pr}_{12}^{*} M_{n, m}-\operatorname{pr}_{13}^{*} M_{n, m}-\pi^{*}\left(\mu_{n, m} \times \mu_{n, m}\right)^{*} A_{n+m}-\int_{I} H^{*} \iota_{n+m}$

is a coboundary, which follows from 47$),(80),(82)$, and $(\diamond)$.

Commutative: Select a homotopy $H: \mu_{m, n} \circ \tau \simeq \mu_{n, m}$ where $\tau=$ flip. In view of the graded commutativity for differential forms, to prove $\tau^{*}\left(\hat{x}_{1} \times \hat{x}_{0}\right)=$ $\hat{x}_{0} \times \hat{x}_{1}$ it remains by (18) and $(19)$ to show that

$$
\begin{gathered}
\tau^{*}\left(B\left(\omega_{1}, \omega_{0}\right)+h_{1} \times \omega_{0}+\omega_{1} \times h_{0}-h_{1} \times \delta h_{0}+\left(c_{1} \times c_{0}\right)^{*} M\right) \\
-\left[B\left(\omega_{0}, \omega_{1}\right)+h_{0} \times \omega_{1}+\omega_{0} \times h_{1}-h_{0} \times \delta h_{1}+\left(c_{0} \times c_{1}\right)^{*} M\right]-\left(c_{0} \times c_{1}\right)^{*} \int_{I} H^{*} \iota
\end{gathered}
$$

bounds. To be able to apply $(\diamond)$ we need to reduce the problem to the space $E_{n} \times E_{m}$. Recall that the cross-product of cochains is not graded 
commutative, but is so up to natural homotopy $D$ from (73). Using (73) and (74) one computes that, up to coboundaries, (A) is

$$
D\left(c_{0}^{*} \iota, c_{1}^{*} \iota\right)+\tau^{*}\left(c_{1} \times c_{0}\right)^{*} M-\left(c_{0} \times c_{1}\right)^{*} M-\left(c_{0} \times c_{1}\right)^{*} \int H^{*} \iota .
$$

In full detail,

$$
\begin{aligned}
& \tau^{*}\left[B\left(\omega_{1}, \omega_{0}\right)+h_{1} \times \omega_{0}+\omega_{1} \times h_{0}-h_{1} \times \delta h_{0}\right]-B\left(\omega_{0}, \omega_{1}\right)-\omega_{0} \times h_{1}-h_{0} \times c_{1}^{*} \iota \\
\equiv & D\left(\omega_{0}, \omega_{1}\right)+\tau^{*}\left(\omega_{1} \times h_{0}-\delta h_{1} \times h_{0}\right)-D\left(\omega_{0}, \delta h_{1}\right)-h_{0} \times c_{1}^{*} \iota \\
\equiv & D\left(\omega_{0}, \omega_{1}\right)+\tau^{*}\left(c_{1}^{*} \iota \times h_{0}\right)-D\left(\omega_{0}, \delta h_{1}\right)-\tau^{*}\left(c_{1}^{*} \iota \times h_{0}\right)-D\left(\delta h_{0}, c_{1}^{*} \iota\right) \\
= & D\left(\omega_{0}, \omega_{1}-\delta h_{1}\right)-D\left(\delta h_{0}, c_{1}^{*} \iota\right)=D\left(c_{0}^{*} \iota, c_{1}^{*} \iota\right) .
\end{aligned}
$$

Now $(B)$ is the pullback along $c_{0} \times c_{1}$ of the cochain

$$
D\left(\iota_{n}, \iota_{m}\right)+\tau^{*} M_{m, n}-M_{n, m}-\int H^{*} \iota_{n+m}
$$

on $E_{n} \times E_{m}$, which bounds by virtue of $(\diamond)$ and $(73), 80$, , 82.

Units: Observe that $B\left(\omega_{\mathrm{pt}},-\right)$ is a natural chain homotopy from $\omega \mapsto \omega_{\mathrm{pt}} \bar{\wedge}$ $\omega=\omega$ to $\omega \mapsto \omega_{\text {pt }} \times \omega=\omega$, as is the zero map. By the Acyclic Models Theorem 7.10 these maps are themselves chain homotopic. In particular $B\left(\omega_{\mathrm{pt}}, \omega_{0}\right)$ is a coboundary for every closed $\omega_{0}$. Now $\hat{1} \times \hat{x}_{0}$ is

$\left[\mu\left(u \times c_{0}\right), \omega_{\mathrm{pt}} \bar{\top} \omega_{0}, B\left(\omega_{\mathrm{pt}}, \omega_{0}\right)+U \times \omega_{0}+\omega_{\mathrm{pt}} \times h_{0}-U \times \delta h_{0}+\left(u \times c_{0}\right)^{*} M\right]$.

Combining the above observation with Lemma 3.10 and using that $\omega_{\mathrm{pt}}$ is the unit for both forms and smooth cochains, this class equals

$$
\left[\mu\left(u \times c_{0}\right), \omega_{0}, U \times c_{0}^{*} \iota+h_{0}+\left(u \times c_{0}\right)^{*} M\right] .
$$

Select a homotopy $H: \mu \circ(u \times$ id $) \simeq$ id. By 18 and $(19)$, to prove $\hat{1} \times \hat{x}_{0}=\hat{x}_{0}$ it suffices to show that the pullback along $c_{0}$ of

$$
U \times \iota_{n}+(u \times \mathrm{id})^{*} M_{0, n}-\int H^{*} \iota_{n}
$$

bounds, which follows from 81 , $82,(\diamond)$.

Associativity: We wish to prove $\hat{x}_{0} \times\left(\hat{x}_{1} \times \hat{x}_{2}\right)=\left(\hat{x}_{0} \times \hat{x}_{1}\right) \times \hat{x}_{2}$. Select a homotopy $H: \mu(\mathrm{id} \times \mu) \simeq \mu(\mu \times \mathrm{id})$. Using the associativity of the exterior product and cross product and combining (18), (19), and Example 7.12 we see that we are reduced to showing that

$$
M_{n, m+l}+\mu_{n, m+l}^{*}\left(\iota_{n} \times M_{m, l}\right)-M_{n+m, l}-\mu_{n+m, l}^{*}\left(M_{n, m} \times \iota_{l}\right)-\int H^{*} \iota_{n+m+l}
$$

bounds, which follows from $(\diamond)$ and $(80)$. 
Proposition 8.8. For smooth maps $f, g$ we have

$$
(f \times g)(\hat{x} \times \hat{y})=f(\hat{x}) \times g(\hat{y}) .
$$

Proof. Straightforward, using naturality of $B, \bar{\wedge}, \delta$, and $\times$.

Combining the previous proposition with Proposition 8.1 gives:

Corollary 8.9. Our functors $\hat{E}^{2 *}$ have been canonically lifted to the category of (graded) commutative unital rings.

\subsubsection{Further Properties}

The map $a$ from Definition 3.11 cannot be multiplicative by reasons of degree. Instead, one demands [BS10] the following compatibility:

Proposition 8.10. For $n, m$ even, $\theta \in \Omega^{n-1}(N ; V), \hat{x}=[c, \omega, h] \in \hat{E}^{m}(M)$ :

$$
a(\theta) \times \hat{x}=a(\theta \bar{\wedge} \hat{x}) .
$$

Proof. Select a homotopy $H: \mu($ const $\times \mathrm{id}) \simeq$ const. Using $B(d \theta, \omega) \equiv$ $\theta \bar{\wedge} \omega-\theta \times \omega$ from (72), (18), and $d \theta \times h \equiv \theta \times \delta h$ we compute:

$$
\begin{aligned}
a(\theta) \times \hat{x} & =\left[\mu(\text { const } \times c), d \theta \bar{\wedge} \omega, B(d \theta, \omega)+\theta \times \omega+d \theta \times h-\theta \times \delta h+(\text { const } \times c)^{*} M\right] \\
& =\left[\mu(\text { const } \times c), d \theta \wedge \omega, \theta \bar{\wedge} \omega+(\text { const } \times c)^{*} M\right]
\end{aligned}
$$

We wish to compare this class with (recall that $\omega$ is automatically closed)

$$
a(\theta \pi R \hat{x})=[\text { const }, d \theta \pi \omega, \theta \bar{\wedge} \omega]
$$

By Lemma 3.10 and Proposition 3.13 it suffices to show that

$$
(\text { const } \times \mathrm{id})^{*} M_{n, m}-\int_{I} H^{*} \iota_{n+m}
$$

is a coboundary, which is follows from $(\diamond)$ and 80 .

We record also the following proposition, which is straightforward from Definitions 8.3, 8.5 (the maps $I, R$ were defined in Definition 3.11): 
Proposition 8.11. The maps $R, I$ preserve the external product and $1_{\mathrm{pt}}$.

Corollary 8.12. The maps $R, I$ are unital natural homomorphisms of graded rings $R: \hat{E}^{2 *} \rightarrow \Omega^{2 *}(-; V), I: \hat{E}^{2 *} \rightarrow E^{2 *}$. We have

$$
a(\theta) \cup \hat{x}=a(\theta \wedge R \hat{x}), \quad \theta \in \Omega^{n-1}(M ; V), \hat{x} \in \hat{E}^{m}(M) .
$$

\subsection{Extension to Odd Degrees}

By demanding compatibility with the integration map (67), the canonical structure in even degrees from the previous section can be uniquely promoted to a product for all degrees. This extension is completely formal and can be carried out for any differential cohomology theory with the following properties:

1. $\hat{E}$ is a differential cohomology theory of pairs (a variant of [BS10]) in the sense of a family of $\mathbf{A b}$-valued functors with natural homomorphisms $a, R, I$ satisfying Theorem 3.12 .

2. $\hat{E}$ admits a long exact sequence of pairs as in Theorem 3.15 .

3. An integration for pairs $\int: \hat{E}^{n+1}\left(M \times S^{1}, M \times 1\right) \rightarrow \hat{E}^{n}(M)$, meaning a natural transformation commuting with $R, I$, and $a$. Theorem 6.15 uses only formal arguments to produce a corresponding 'absolute' integration map, for which we assume the property of Proposition 6.18.

4. A natural, graded commutative product-structure in even degrees for which $R, I$ are homomorphisms and $a$ satisfies (83). For the associated external product we require Lemma 8.18 .

The transition is based on the following variant of [BS10, p.21]:

Proposition 8.13. For any $\hat{x} \in \hat{E}^{n}(M)$ there is $\hat{X} \in \hat{E}^{n+1}\left(M \times S^{1}, M \times 1\right)$ with

$$
\int \hat{X}=\hat{x}
$$

Any two choices of $\hat{X}$ differ by a $(\theta)$, where $\theta \in \Omega^{n}\left(M \times S^{1}, M \times 1\right)$ is such that $\int \theta$ represents an element in the image of the Chern character. 
Proof. Existence. By the surjectivity of

$$
\hat{E}^{n}\left(M \times S^{1}, M \times 1\right) \rightarrow E^{n}\left(M \times S^{1}, M \times 1\right) \cong E^{n-1}(M),
$$

we may choose $\hat{X} \in \hat{E}^{n}\left(M \times S^{1}, M \times 1\right)$ with $\int I(\hat{X})=I(\hat{x})$. By Proposition 6.16, $\hat{x}-\int \hat{X}$ lies in the kernel of $I$ and therefore equals $a(\theta)$ for some $\theta \in \Omega^{n-2}(M ; V)$, by Theorem 3.12. Pick $\alpha: S^{1} \rightarrow \mathbb{R}$ smooth with $\int \alpha(t) d t=1$ and $\alpha(1)=0$ and let $\tilde{\theta}=\operatorname{pr}_{1}^{*} \theta \wedge \alpha(t) d t \in \Omega^{n-1}\left(M \times S^{1}, M \times 1 ; V\right)$. Then $\int \tilde{\theta}=\theta$, and $\hat{X}+a(\tilde{\theta})$ is the element we seek.

Uniqueness. If $\int \hat{X}=\int \hat{X}^{\prime}$, then Proposition 6.16 implies $\int I\left(\hat{X}-\hat{X}^{\prime}\right)=0$ so that $I\left(\hat{X}-\hat{X}^{\prime}\right)$ lies in the kernel of the suspension (right isomorphism in (84)), which is trivial. It follows that $I\left(\hat{X}-\hat{X}^{\prime}\right)=0$, whence $\hat{X}-\hat{X}^{\prime}=a(\theta)$ for some $\theta \in \Omega^{n-1}\left(M \times S^{1}, M \times 1 ; V\right)$. Since $a \int \theta=\int a(\theta)=\int\left(\hat{X}-\hat{X}^{\prime}\right)=0$, Theorem 3.12 implies that $\int \theta$ lies in the image of the Chern character.

We will generally denote a choice of element $\hat{X}$ with $\int \hat{X}=\hat{x}$ as in 84 by upper case.

Definition 8.14. For $\hat{x} \in \hat{E}^{n}(M), \hat{y} \in \hat{E}^{m}(N)$ pick classes $\hat{X} \in \hat{E}^{n+1}(M \times$ $\left.S^{1}\right), \hat{Y} \in \hat{E}^{m+1}\left(N \times S^{1}\right)$ with $\int \hat{X}=\hat{x}, \int \hat{Y}=\hat{y}$. Define

$$
\hat{x} \times \hat{y}= \begin{cases}\int \hat{X} \times \hat{y} & (\text { for } n \text { odd }, m \text { even }), \\ \int \hat{x} \times \hat{Y} & (\text { for } n \text { even, } m \text { odd }), \\ \int \hat{x} \times \hat{Y}=-\int \hat{X} \times \hat{y} & (\text { for } n, m \text { odd }) .\end{cases}
$$

The last equality follows from Proposition 6.18.

$$
\int \hat{X} \times \hat{y} \stackrel{\text { def }}{=} \iint \hat{X} \times \hat{Y}=-\iint^{\prime} \hat{X} \times \hat{Y} \stackrel{\text { def }}{=}-\int \hat{x} \times \hat{Y}
$$

Lemma 8.15. The definition of $\hat{x} \times \hat{y}$ is independent of the choices of $\hat{X}$ and $\hat{Y}$. Moreover, in all degrees we have

$$
a(\theta) \times \hat{x}=a(\theta \pi R \hat{x}) .
$$

Proof. Suppose, for example, that $\int \hat{X}=\hat{x}=\int \hat{X}^{\prime}$ in the case $n$ odd, $m$ even. We will use the maps

$$
j: M \times S^{1} \rightarrow\left(M \times S^{1}, M \times 1\right), \quad i: M \times 1 \longleftrightarrow M \times S^{1}: \mathrm{pr}_{1} .
$$


Using Theorem 3.15, write

$$
j^{*} \hat{Z}=\hat{X}-\operatorname{pr}_{1}^{*} i^{*} \hat{X}, \quad j^{*} \hat{Z}^{\prime}=\hat{X}^{\prime}-\operatorname{pr}_{1}^{*} i^{*} \hat{X}^{\prime}
$$

for $\hat{Z}, \hat{Z}^{\prime} \in \hat{E}^{n+1}\left(M \times S^{1}, M \times 1\right)$. By Theorem 6.15 and Proposition 8.13 . $\hat{Z}-\hat{Z}^{\prime}=a(\theta)$ for some form $\theta \in \Omega^{n}\left(M \times S^{1}, M \times 1\right)$ with $\int \theta$ in the image of the Chern character. Since $R$ has image in can(im(ch)) and ch is multiplicative, $\int \theta \bar{\wedge} \hat{y}$ also represents an element in the image of the Chern character. By Proposition 6.16.

$$
\int a(\theta) \times \hat{y} \stackrel{83}{=} \int a(\theta \pi R \hat{y})=a\left(\int \theta \pi R \hat{y}\right)=0,
$$

which shows that $\hat{x} \times \hat{y}$ is well-defined:

$$
\int\left(\hat{X}-\hat{X}^{\prime}\right) \times \hat{y}=\int\left(\hat{Z}-\hat{Z}^{\prime}\right) \times \hat{y}=\int a(\theta) \times \hat{y}=0
$$

Suppose that $\theta \in \Omega^{n-1}(M ; V)$. Still in the case $n$ odd, $m$ even we wish to prove 85$)$. Pick $\tilde{\theta} \in \Omega^{n}\left(M \times S^{1} ; V\right)$ with $\int \tilde{\theta}=\theta$. Then $\int a(\tilde{\theta})=a(\theta)$, so that by definition

$$
a(\theta) \times \hat{y} \stackrel{\text { def }}{=} \int a(\tilde{\theta}) \times \hat{y} \stackrel{83}{=} \int a(\tilde{\theta} \bar{\wedge} R \hat{y})=a\left(\int \tilde{\theta} \bar{\wedge} R \hat{y}\right)=a(\theta \bar{\wedge} R \hat{y}) .
$$

The proofs of the other two cases are completely parallel.

The following is an immediate consequence of the well-definedness of Definition 8.14 and of the corresponding property in even degrees:

Proposition 8.16. The product is bilinear, unital, and graded commutative in all degrees.

Associativity follows directly from the following compatibility of multiplication and the integration map:

Proposition 8.17. For any $\hat{x} \in \hat{E}^{n}\left(M \times S^{1}\right)$ and $\hat{y} \in \hat{E}^{m}(N)$ we have

$$
\int(\hat{y} \times \hat{x})=\hat{y} \times\left(\int \hat{x}\right), \quad(-1)^{|\hat{y}|} \int(\hat{x} \times \hat{y})=\left(\int \hat{x}\right) \times \hat{y} .
$$

Proof. The second formula follows from the first by graded commutativity. Considering each of the cases in Definition 8.14, the first formula follows from the next lemma, which is the corresponding result in even degrees. 
Lemma 8.18. For $\hat{x} \in \hat{E}^{n}(M), \hat{y} \in \hat{E}^{m}\left(N \times S^{1} \times S^{1}\right)$ and $n, m$ even we have

$$
\iint(\hat{x} \times \hat{y})=\hat{x} \times\left(\iint \hat{y}\right) .
$$

Proof. The proof is similar to that of Proposition 6.18 and we will use the notation established there. Pick $\hat{y}^{\prime} \in \hat{E}^{n}\left(M \times S^{1} \times S^{1}, M \times 1 \times S^{1}\right)$ with $j_{2}^{*} \hat{y}^{\prime}=\hat{y}-\operatorname{pr}_{2}^{*} i_{2}^{*} \hat{y}$. Then

$$
\hat{y}^{\prime}-\operatorname{pr}_{1}^{\prime *} i_{1}^{\prime *} \hat{y}^{\prime}=\left[c_{0}, \omega_{0}, h_{0}\right]
$$

may represented as a triple as in Lemma 6.19. Since such triples may be viewed as relative classes as required by (69), the double integral may be performed 'directly' (without choosing a replacement to compute the second integration): as in the proof of Proposition 6.18, Theorem 6.15 implies

$$
\iint \hat{y}=\iint \hat{y}^{\prime}=\iint\left[c_{0}, \omega_{0}, h_{0}\right]=\left[\iint c_{0}, \iint \omega_{0}, \iint h_{0}\right]
$$

and similarly

$$
\iint \hat{x} \times \hat{y}=\left[\iint \mu\left(c_{0} \times c_{1}\right), \iint \omega_{0} \bar{\wedge} \omega_{1}, \iint\left(B\left(\omega_{0}, \omega_{1}\right)+\omega_{0} \times h_{1}+h_{1} \times \omega_{1}-h_{0} \times \delta h_{1}+\left(c_{0} \times c_{1}\right)^{*} M\right)\right] .
$$

We have to compare this class with $\hat{x} \times\left(\iint \hat{y}\right)$, which is

$$
\left[\mu\left(c_{0} \times \iint c_{1}\right), \omega_{0} \pi\left(\iint \omega_{1}\right), B\left(\omega_{0}, \iint \omega_{1}\right)+h_{0} \times\left(\iint \omega_{1}\right)+\omega_{0} \times\left(\iint h_{1}\right)-h_{0} \times \delta\left(\iint h_{1}\right)+\left(c_{0} \times \iint c_{1}\right)^{*} M\right] .
$$

Example 7.15 implies that $\iint B(-,-)$ and $B\left(-, \iint-\right)$ differ on closed forms only by a coboundary. Select a homotopy $H$ from $\mu_{n, m} \circ\left(\mathrm{id}_{n} \times \varepsilon_{m-1} \circ \Sigma \varepsilon_{m-2}\right)$ to $\varepsilon_{n+m-1} \circ \Sigma \varepsilon_{n+m-2} \circ \Sigma^{2} \mu_{n, m-2}$. Then by Lemma 3.10 and Proposition 3.13 it suffices to show that the pullback under $\left(c_{0} \times \iint c_{1}\right)$ of

$$
\iint\left(\operatorname{id}_{n} \times \varepsilon_{m-1} \circ \Sigma \varepsilon_{m-2}\right)^{*} M_{n, m}-\int_{I} H^{*}\left(\left(\Omega \varepsilon_{n+m-1}^{\mathrm{adj}}\right)^{-1}\right)^{*}\left(\left(\varepsilon_{n+m-2}^{\mathrm{adj}}\right)^{-1}\right)^{*} \iota_{n+m-2}-M_{n, m-2}
$$

is a coboundary, which follows from $(\diamond)$.

Theorem 8.19. Let $E$ be a rationally even, multiplicative cohomology theory and choose an $\Omega$-spectrum $E_{n}$ representing it. Then the Hopkins-Singer differential extension $\hat{E}$ can canonically be viewed as a multiplicative differential cohomology theory in the sense of [BS10]. The product structure is compatible with integration.

Proof. It remains only to remark that multiplicativity of $f^{*}$ in odd degrees follows from Proposition 8.8 and the functoriality in Theorem 6.15. 


\section{Highly Structured Differential FunC- TION SPECTRA}

We have shown that for rationally even spectra $E$ up to homotopy it is possible to construct a canonical product structure on the differential cohomology groups. This is slightly unsatisfactory for two reasons:

1. Even though most naturally arising cohomology theories of interest are rationally even (with the exception of algebraic $K$-theory), this is a rather technical condition. An explanation for this condition is that it seems to guarantee a unique multiplicative structure, at least if one requires compatibility with the integration maps.

2. In the field theory interpretation of differential cohomology (Chapter 11), the groups $\hat{E}^{n}(M)$ are the equivalence classes of gauge potentials (of fields whose charges have been lifted along the generalized Chern character). Important operations like gluing fields or integrating them on manifolds with boundary do not descend to equivalence classes.

The second point motivates looking for a refined structure that represents differential cohomology

$$
\hat{E}^{*}: \operatorname{Man}^{\mathrm{op}} \longrightarrow \mathbb{Z} \text {-Graded-Rings. }
$$

This refined structure will be a functor of $\infty$-categories

$$
N \mathrm{Man}^{\text {op }} \rightarrow \mathrm{CAlg}(\mathrm{Sp}) .
$$

Here, NMan denotes the ordinary nerve [GJ09, Example I.1.4] of the category of manifolds, that is, the simplicial set whose set of $n$-simplices is that of all composable sequences

$$
M_{0} \stackrel{f_{1}}{\rightarrow} \cdots \stackrel{f_{n}}{\longrightarrow} M_{n}
$$

of manifolds $M_{i}$ and smooth maps $f_{i}$. The notation from higher algebra is explained in the next subsection.

The study of differential function spectra was initiated in [HS05, 4.6], where essentially an ordinary functor

$$
\operatorname{Man}^{\mathrm{op}} \rightarrow \mathbf{h S p}, \quad S \mapsto \operatorname{filt}_{n}\left(E_{n} ; \iota_{n}\right)^{S}
$$


is constructed 8

Beginning with a 'highly structured' commutative ring spectrum $E \in$ $\mathrm{CAlg}(\mathrm{Sp})$, we will discuss how one may construct refinements (86). By developing the deRham isomormophism for Eilenberg-Mac Lane spectra and the theory of smooth objects ( $\infty$-category valued presheaves on manifolds), Bun12 exhibits a passage from multiplicative differential data to functors (86) (discussed in Section 9.1). While several examples of multiplicative data are given, Bun12] does unfortunately not show how to obtain such multiplicative data for generic $E$. This is certainly a crucial issue because the deRham homomorphism is essentially just a feature of ordinary Eilenberg-Mac Lane spectra, while the coherence conditions (similar to the compatibility condition (80) ) are encoded in the multiplicative data.

The main point that remains is therefore the construction of canonical multiplicative data for $E \in \mathrm{CAlg}(\mathrm{Sp})$, that is, an equivalence class of a morphism in $\mathrm{CAlg}(\mathrm{Sp})$

$$
E \wedge H \mathbb{R} \rightarrow H V, \quad V_{*}=\pi_{*}(E) \otimes_{\mathbb{Z}} \mathbb{R},
$$

which will then lead to our refinement 86 . Let me point out that the construction of such $E_{\infty}$-morphisms is daunting business and hardly possible 'by hand.' We will use the Schwede-Shipley Theorem [SS03a and the idea that on certain Eilenberg-Mac Lane spectra there is an essentially unique multiplicative structure. This theorem forces us to restrict to cofibrant $V$. The results in this chapter are therefore not a generalization of the rationally even case.

We will establish $(88)$ in $(97)$ and call it a refined Chern character.

\subsection{Construction from Multiplicative Data}

\subsubsection{Notation from Higher Algebra}

A short introduction to $\infty$-categories in general is given in the appendix. Here we will recall briefly some terminology from 'higher algebra' [Lur11. We will generally use the notation introduced there and in [Lur09].

\footnotetext{
${ }^{8} \mathrm{Up}$ to the issue of functorial replacement in (4.41) which, strictly speaking, involves a fibrant replacement in a diagram category - in particular, the spaces filt ${ }_{n}\left(E_{n} ; \iota_{n}\right)^{S}$ are replaced by equivalent spaces (which of course makes no matter upon the passage to homotopy groups).
} 
We let $\Gamma$ denote Segal's (skeletal) category of finite pointed sets. This category has objects

$$
0 \in\langle n\rangle=\{0, \ldots, n\}, \quad n \geq 0,
$$

base pointed by zero. Morphisms $\alpha:\langle m\rangle \rightarrow\langle n\rangle$ are ordinary maps preserving the base point. For example we have 'characteristic maps'

$$
\chi^{i}:\langle n\rangle \rightarrow\langle 1\rangle, \quad j \mapsto \begin{cases}1 & (i=j), \\ 0 & \text { (else) } .\end{cases}
$$

A morphism is said to be inert if $\alpha^{-1}(i)$ is a singleton for every $i \neq 0$.

The following is a different way to view an ordinary monoidal category: it is a category $\mathscr{C}$ along with a way to 'operate' on sequences

$$
\left(C_{1}, \ldots, C_{n}\right)
$$

of objects of $\mathscr{C}$. That is, a monoidal structure gives a way to 'contract' and 'expand' such sequences, for example,

$$
(U, V, W, X, Y, Z) \longrightarrow\left(U, V \otimes W, 1_{\mathscr{C}}, X \otimes Y\right) .
$$

Of course, we may apply more contractions on the right. Similar to an operad, this should be compatible in the sense that the result remains the same if we compute some intermediate step first. The precise way to state this is that the category of sequences $\mathcal{C}^{\otimes}$ is the total space of a Grothendieck opfibration $\mathrm{C}^{\otimes} \rightarrow N \Delta^{\mathrm{op}}$ (the classical counterpart of a coCartesian fibration). The shape of $\Delta^{\mathrm{op}}$ encodes the compatibilities we require. The operation 89 is then an allowed 'horizontal lift' of the morphism $\alpha:[4] \rightarrow[6]$ in $\Delta$ given by $0 \leq 1 \leq 3 \leq 3 \leq 5$.

In the symmetric monoidal case, $\Delta^{\mathrm{op}}$ is replaced by $\Gamma$ and morphisms $\alpha:\langle m\rangle \rightarrow\langle n\rangle$ in $\Gamma$ are thought of as contractions

$$
\left(C_{1}, \ldots, C_{m}\right) \rightarrow\left(\bigotimes_{i \in \alpha^{-1}(1)} C_{i}, \ldots, \bigotimes_{i \in \alpha^{-1}(n)} C_{i}\right) .
$$

The compatibility mentioned above is of course only required up to coherent homotopy: 
Definition 9.1 (1.2.12 in [Lur07b]). A symmetric monoidal $\infty$-category is a coCartesian fibration

$$
p: \mathfrak{C}^{\otimes} \rightarrow N \Gamma
$$

with the additional property that for each $n \geq 0$ the canonical map induced by 'fiber transport' along $\chi^{1}, \ldots, \chi^{n}$ (well-defined up to equivalence)

$$
\mathrm{e}_{\langle n\rangle}^{\otimes} \rightarrow \mathrm{e}_{\langle 1\rangle} \times \cdots \times \mathcal{C}_{\langle 1\rangle}
$$

is a categorical equivalence. The fiber $\mathcal{C}=\mathcal{C}_{\langle 1\rangle}$ is called the underlying $\infty$-category.

Example 9.2. The $\infty$-category of spectra $\mathrm{Sp}$ is defined as the full subcategory of $\operatorname{Fun}\left(\mathbb{Z} \times \mathbb{Z}, \mathcal{S}^{*}\right)$ spanned by the spectrum objects $X$ (that is, $X_{i j} \simeq \Delta^{0}$ for all $i \neq j$ and the diagram $X$ induces identifications $\left.X_{i, i} \simeq \Omega X_{i+1, i+1}\right)$. There exists a symmetric monoidal structure on $\mathrm{Sp}$. This is the main result of [Lur07a] and Lur07b].

Example 9.3. The derived category $\mathcal{D}(\mathscr{A})$ [Lur11, 1.3.5.8] of an abelian category $\mathscr{A}$ (for example, the category of $R$-modules $\operatorname{Mod}_{R}$ for an ordinary ring $R$ ). The monoidal structure stems from the tensor product of chain complexes.

In more detail, the unbounded derived category is defined as the 'differential graded nerve' [Lur11, 1.3.1.6] of the differential graded category of real (unbounded) chain complexes $\mathbf{C h}_{\mathbb{R}}$. The differential enrichment is given by the internal hom, i.e. the chain complex with $n$-chains

$$
\operatorname{Map}_{\mathbf{C h}}(C, D)_{n}=\prod_{i \in \mathbb{Z}} \operatorname{Vect}_{\mathbb{R}}\left(C_{i}, D_{i+n}\right) .
$$

Thus, $\mathrm{N}_{\mathrm{dg}}(\mathbf{C h})=\mathcal{D}\left(\right.$ Vect $\left._{\mathbb{R}}\right)$. By [Lur11, Remark 1.3.1.11], the homotopy category of a differential graded category (obtained by passing the the homology of the mapping chain complexes) is canonically isomorphic to the homotopy category of the $\infty$-category obtained by passing to the differential graded nerve.

Definition 9.4 (1.3.3, 1.2.1, and 1.1.27 in [Lur07b]). Let $p: \mathcal{C}^{\otimes} \rightarrow N \Gamma$ be a symmetric monoidal $\infty$-category. The $\infty$-category of commutative algebra objects $\operatorname{CAlg}(\mathcal{C})$ is the full subcategory of $\operatorname{Fun}\left(N \Gamma, \mathfrak{C}^{\otimes}\right)$ spanned by all 
sections $A: N \Gamma \rightarrow \mathrm{C}^{\otimes}$ of $p$ which carry inert morphisms to $p$-coCartesian edges.

Definition 9.5. Suppose $p: \mathcal{C}^{\otimes} \rightarrow N \Gamma$ and $q: \mathcal{D}^{\otimes} \rightarrow N \Gamma$ are symmetric monoidal $\infty$-categories. A symmetric monoidal functor is a simplicial map $f: \mathfrak{C}^{\otimes} \rightarrow \mathcal{D}^{\otimes}$ with the property that $f q=p$ and which preserves coCartesian edges. We will often identify it with its restriction $f: \mathcal{C} \rightarrow \mathcal{D}$ to the fiber over $\langle 1\rangle$.

In addition, for each $E \in \mathrm{CAlg}(\mathrm{Sp})$ we may form the $\infty$-category of $E$ modules $\operatorname{Mod}_{E}$, which is again symmetric monoidal. The corresponding $\infty$ category of algebras $\mathrm{CAlg}\left(\operatorname{Mod}_{E}\right)$ is called the $\infty$-category of commutative $E$-algebra objects. We will write $\mathrm{CAlg}_{R}=\mathrm{CAlg}\left(\operatorname{Mod}_{H R}\right)$ for an ordinary $\operatorname{ring} R$.

\subsubsection{Smash Product of Spectra}

Recall from [Lur07a, 4.1.1] that the $\infty$-category of all $\infty$-categories Cat $_{\infty}$ may be viewed as the simplicial nerve of the fibrant-cofibrant objects of Set $_{\Delta}^{+}$. Since $\operatorname{Set}_{\Delta}^{+}$is even a monoidal model category (for the Cartesian product of simplicial sets), we may define a monoidal structure $\mathcal{C} a t_{\infty}^{\otimes}$ on $\mathrm{C}_{\infty}$ with objects

$$
\left[X_{1}, \ldots, X_{n}\right], \quad X_{i} \in \mathcal{C} a t^{\infty}, \quad n \geq 0
$$

An edge in $\mathcal{C} a t_{\infty}^{\otimes}$ from $\left[X_{1}, \ldots, X_{n}\right]$ to $\left[Y_{1}, \ldots, Y_{m}\right]$ is given by $f \in \Delta([m],[n])$ together with functors

$$
\eta_{i}: X_{f(i-1)+1} \times \cdots \times X_{f(i)} \rightarrow Y_{i}
$$

This is in fact a Cartesian model structure on Cat $t_{\infty}$. The $\infty$-category of algebra objects $\operatorname{Alg}\left({\mathcal{C} a t_{\infty}}\right)$ may be identified with the $\infty$-category $\mathrm{Cat}_{\infty}^{\text {Mon }}$ of all monoidal $\infty$-categories and whose morphisms are monoidal functors, but the mechanism is unfortunately somewhat abstract (it is basically an unstraightening construction).

We let $\mathrm{C}_{\infty} t_{\infty}^{\mathrm{Pr}}$ denote the subcategory of $\mathrm{C}_{\infty}$ spanned by presentable $\infty$-categories and whose morphisms are required to be colimit-preserving.

It is an important fact that the subcategory $\mathcal{C}_{a} t_{\infty}^{\mathrm{Pr}}{ }^{\otimes} \subset \mathcal{C} a t_{\infty}^{\otimes}$ spanned by objects only those tuples 90 consisting of presentable $\infty$-categories and 
edges (91) that preserve colimits in each variable (are multilinear) determines a monoidal structure on $\mathrm{C} a t_{\infty}^{\mathrm{Pr}}$. The main observation is that the $q$ -

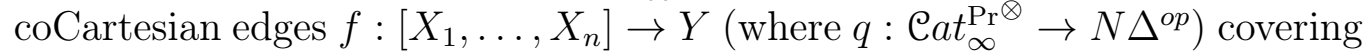
$[n] \rightarrow[1]$ in $\Delta^{o p}$ may be characterized by the following condition:

" $f$ is multilinear and induces for every $Z \in \mathcal{C} a t_{\infty}^{\mathrm{Pr}}$ a categorical equivalence $f^{*}: \operatorname{Fun}^{\mathrm{L}}(Y, Z) \rightarrow \operatorname{Fun}^{\prime}\left(X_{1} \times \cdots \times X_{n}, Z\right)$ onto the full subcategory of multilinear functors."

We even have a canonical choice of $q$-coCartesian morphism covering the edge $[n] \stackrel{\{0, n\}}{\longrightarrow}[1]$ in $\Delta^{o p}$ :

$$
X_{1} \times \cdots \times X_{n} \rightarrow \operatorname{Fun}^{\mathrm{R}}\left(X_{1}^{o p}, \operatorname{Fun}^{\mathrm{R}}\left(X_{2}^{o p}, \ldots \operatorname{Fun}^{\mathrm{R}}\left(X_{n-1}^{o p}, X_{n}\right)\right)\right)
$$

In particular, $\mathcal{S}$ is the unit [Lur11, 5.1.5.6] (the $q$-coCartesian edge []$\rightarrow[\mathcal{S}]$ is given by $\Delta^{0} \stackrel{\Delta^{0}}{\longrightarrow} \mathcal{S}$ ) and we are thus not dealing with a Cartesian monoidal structure (since $\mathcal{S}$ is not final). By [Lur07a, 1.4.3], the unit $\mathcal{S}$ determines an essentially well-defined object of $\operatorname{Alg}\left(\mathrm{C} a t_{\infty}^{\mathrm{Pr}}\right)$, which determines a monoidal structure on $\mathcal{S}$. This is just the Cartesian structure.

Now let $\mathrm{C} a t_{\infty}^{\mathrm{Pr}, \sigma^{\otimes}} \subset \mathcal{C} a t_{\infty}^{\mathrm{Pr} \otimes}$ denote the full subcategory whose objects are tuples $(90)$ consisting only of stable presentable $\infty$-categories. This will define a monoidal structure on $\mathrm{C} a t_{\infty}^{\mathrm{Pr}, \sigma}$, owing to the fact that this is a localization of $\mathrm{C} a t_{\infty}^{\mathrm{Pr}}$ whose localization functor

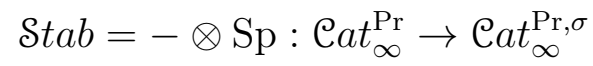

is compatible with the monoidal structure [Lur07a, 1.3.9]. By the same result, Stab extends to a monoidal functor while the inclusion extends to a lax monoidal functor.

In particular, $\mathrm{Sp}=\mathcal{S} \operatorname{tab}(\mathcal{S})$ is the unit. By [Lur07a, 1.4.3], the unit Sp determines an essentially well-defined object of $\operatorname{Alg}\left(\mathcal{C} a t_{\infty}^{\mathrm{Pr}, \sigma}\right)$ - by the mechanism of left Kan extension. As remarked to above, performing then an unstraightening construction finally yields a monoidal structure on Sp, called the smash product.

\subsubsection{Homotopy Pullback Construction}

In view of our discussion of products in the rationally even case, it is clear that their existence depends on a coherent choice of maps $\mu_{n, m}$ representing multiplication and cochains $M_{n, m}$ relating the fundamental cocycles. 
The passage from multiplicative data to differential function spectra has been made explicit in [Bun12], some results of which we now briefly recall.

Definition 9.6. A canonical multiplicative differential datum for $E \in \mathrm{CAlg}(\mathrm{Sp})$ is a morphism $\left(V^{*}=\pi_{-*} E \otimes_{\mathbb{Z}} \mathbb{R}\right.$ denotes the coefficients of $E$ )

$$
c: E \wedge H \mathbb{R} \rightarrow H V
$$

in the $\infty$-category $\mathrm{CAlg}(\mathrm{Sp})$.

The (generalized) Eilenberg-Mac Lane spaces $H V$ above are reviewed in subsection 9.2.1.

Roughly speaking, the differential function spectrum is the (homotopy) pullback in Sp of the diagram

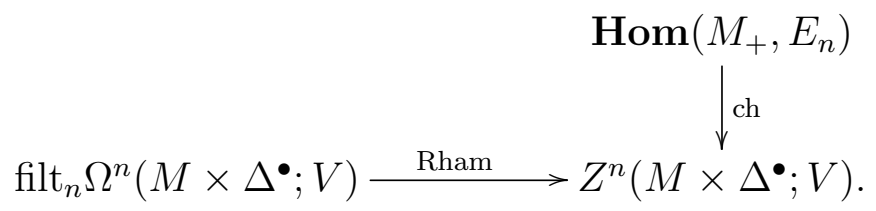

Here, $Z\left(M \times \Delta^{\bullet} ; V\right)$ denotes the cocycle spectrum (34), which we have evaluated at $M_{+}$, and ch is the map (40), viewed as a morphism in Sp. The $n$-th level of the lower left corner is

$$
\text { filt }_{n} \Omega^{n}\left(M \times \Delta^{\bullet} ; V\right)=\prod_{i \in \mathbb{Z}} \text { filt }_{n} \Omega^{i}\left(M \times \Delta^{\bullet} ; V^{n-i}\right),
$$

where filt $_{n}$ indicates that we take only forms whose Künneth component in direction of $\Delta^{\bullet}$ is of degree $\leq n$. The lower horizontal map is given by viewing a differential form as a cochain (the 'deRham homomorphism'). As shown in [HS05, there is an equivalence

$$
Z\left(M \times \Delta^{\bullet} ; V\right) \simeq \operatorname{Hom}\left(M_{+}, H V\right),
$$

so one may replace the lower right corner in 92 by a mapping spectrum. For each $M$, we wish to refine the resulting diagram to $\mathrm{CAlg}(\mathrm{Sp})$. Passing to mapping spectra and precomposing with $E \rightarrow E \wedge H \mathbb{R}$, a multiplicative datum $(E, V, c)$ gives rise to a diagram

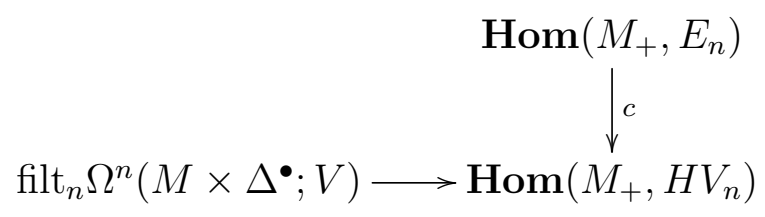


whose vertical map is a morphism in $\mathrm{CAlg}(\mathrm{Sp})$. The main result of [Bun12, Section 4] is a version of deRham's Theorem for Eilenberg-Mac Lane spectra. In particular, a morphism in $\mathrm{CAlg}(\mathrm{Sp})$ is constructed that replaces the horizontal map in the previous diagram. For varying manifolds $M$, the result may be viewed as a diagram in the $\infty$-category

$$
\operatorname{Fun}\left(N(\operatorname{Man})^{\text {op }}, \mathrm{CAlg}(\mathrm{Sp})\right),
$$

of commutative ring spectra-valued presheaves on manifolds (in Bun12, this category is denoted by $\mathbf{S m}\left(\operatorname{CommMon}\left(\mathrm{N}(\mathbf{S p})\left[W^{-1}\right]^{\wedge}\right)\right.$, but we stick to the notation of [Lur11]).

The limit of this diagram then gives (86). The construction therefore has two components:

1. The horizontal deRham homomorphism must be a morphism in $\mathrm{CAlg}(\mathrm{Sp})$.

2. A morphism $E \rightarrow E \wedge H \mathbb{R} \rightarrow H V$ in $\mathrm{CAlg}(\mathrm{Sp})$, which yields the vertical morphism upon passage to mapping spectra.

A treatment of a candidate for 1. is given in Bun12, Lemma 4.111 and the surrounding discussion]. On the other hand, the morphism in 2. is assumed there as additional data (the multiplicative differential data).

\subsection{Canonical Multiplicative Data}

In this section we will construct canonical multiplicative data (88), assuming that $V$ is a cofibrant commutative DGA.

Recall that the category cDGA of commutative differential graded algebras over the reals admits a model structure whose weak equivalences are the quasi-isomorphisms and whose fibrations are morphisms that are surjective in every degree [Lur11, Proposition 8.1.4.10], [SS00]. While every object is fibrant, in order to get the Schwede-Shipley Theorem going (recalled below), we need to assume that $V \in \mathbf{c D G A}$ is cofibrant.

Our construction of 88 will be based on a generalization of a Theorem of Dold:

Theorem 2 and Proposition 2 in [Dol62] Let E, F be cohomology theories and assume that $F$ takes values in real vector spaces. Then any transformation $\phi: \pi_{*} E \rightarrow \pi_{*} F$ of the coefficients may uniquely be extended to a transformation $\Phi: E \rightarrow F$ of cohomology theories. 
Moreover, if E, F are both multiplicative cohomology theories and $\phi$ is multiplicative, then $\Phi$ is a multiplicative transformation.

While the first part goes through essentially unaltered, we will only be able to prove a restricted version of the second assertion.

\subsubsection{Generalized Eilenberg-Mac Lane spectra}

The following is a $\infty$-categorical formulation of the Schwede-Shipley Comparison Theorem [SS03a]:

Theorem 8.1.2.13 in [Lur11] We have an equivalence of symmetric monoidal $\infty$-categories

$$
\theta: \mathcal{D}\left(\operatorname{Vect}_{\mathbb{R}}\right) \stackrel{\sim}{\longrightarrow} \operatorname{Mod}_{H \mathbb{R}} .
$$

In particular, $\theta$ induces an (ordinary) symmetric monoidal equivalence of the associated homotopy categories.

Both $\mathcal{D}\left(\operatorname{Vect}_{\mathbb{R}}\right)$ and $\operatorname{Mod}_{H \mathbb{R}}$ are stable $\infty$-categories Lur07c which implies in particular that their homotopy categories are additive. This is because suspension is an equivalence in stable $\infty$-categories and characterized by an equivalence of simplicial sets

$$
\operatorname{Map}_{\mathcal{C}}(\Sigma C, D) \simeq \operatorname{RMap}_{\mathcal{C}}(C, D)
$$

and the homotopy groups of a two-fold loop space are abelian groups. Since $\theta$ is an equivalence, it preserves colimits and limits. In particular, it is an exact functor in the sense of [Lur07c, Section 5]. As such, $\theta$ carries suspensions to suspensions and therefore induces and additive functor

$$
\mathrm{h} \theta: \mathrm{h} \mathcal{D}\left(\operatorname{Vect}_{\mathbb{R}}\right) \stackrel{\sim}{\longrightarrow} \mathrm{hMod}_{H \mathbb{R}}
$$

between the homotopy categories, which also inherits a symmetric monoidal structure.

Remark 9.7. The functor $\theta$ is a generalized Eilenberg-MacLane functor. Indeed, for a graded vector space $V$ (regarded as a chain complex with zero differential) we have $\theta(V)=\theta\left(\prod_{i \in \mathbb{Z}} V_{i}[i]\right)=\prod_{i \in \mathbb{Z}} H V_{i}[i]$. We will also write $H V:=\theta(V) \in \operatorname{Mod}_{H \mathbb{R}}$. Here, '[]' denotes the shift. 
To explain what an Eilenberg-Mac Lane spectrum associated to a chain complex is, note that, up to equivalence, a chain map $C_{*}(X) \rightarrow V$ amounts to a map $\Sigma^{\infty} X \rightarrow H V$. In the same way, an Eilenberg-Mac Lane spectrum for a chain complex $D_{*}$ should classify chain maps $C_{*}(X) \rightarrow D_{*}$.

Both the homology groups of a chain complex and the homotopy groups of a spectrum have a description in terms of a Hom-set from the unit objects: for $E \in \operatorname{Mod}_{H \mathbb{R}}$ we have

$$
\begin{aligned}
\pi_{n} E & =\pi_{0} \operatorname{Map}_{\mathrm{Sp}}(\mathbb{S}[n], E)=\mathrm{hSp}(\mathbb{S}[n], E) \\
& \cong \operatorname{hMod}_{H \mathbb{R}}(\mathbb{S} \wedge H \mathbb{R}[n], E) \cong \operatorname{hMod}_{H \mathbb{R}}(H \mathbb{R}[n], E) .
\end{aligned}
$$

Here we have used that the forgetful functor hMod $\operatorname{HR}_{\mathbb{R}} \rightarrow \mathrm{hSp}$ has the left adjoint $-\wedge H \mathbb{R}$. Similarly, for $C \in \mathcal{D}\left(\right.$ Vect $\left._{\mathbb{R}}\right)$ we have

$$
H_{n} C=\pi_{0} \operatorname{Map}_{\mathcal{D}\left(\operatorname{Vect}_{\mathbb{R}}\right)}(\mathbb{R}[n], C)=\mathrm{h} \mathcal{D}\left(\operatorname{Vect}_{\mathbb{R}}\right)(\mathbb{R}[n], C),
$$

where we view $\mathbb{R}$ as a chain complex concentrated in degree 0 .

Lemma 9.8. For every chain complex $C \in \mathcal{D}\left(\right.$ Vect $\left._{\mathbb{R}}\right), \theta$ induces a natural isomorphism of graded vector spaces

$$
\mathrm{h} \theta: H_{*} C \stackrel{\cong}{\longrightarrow} \pi_{*} \theta(C)
$$

If $C \in \mathbf{c D G A}$, this is an isomorphism of graded commutative algebras.

Proof. The functor $\mathrm{h} \theta$ takes the unit $\mathbb{R}$ to the unit in $\operatorname{Mod}_{H \mathbb{R}}$, that is, an Eilenberg-MacLane spectrum $H \mathbb{R}:=\theta(\mathbb{R})$. Since $\mathrm{h} \theta$ is exact, it preserves suspensions and therefore induces an isomorphism of abelian groups

$$
\mathrm{h} \theta: \mathrm{h} \mathcal{D}\left(\operatorname{Vect}_{\mathbb{R}}\right)(\mathbb{R}[n], C) \rightarrow \operatorname{hMod}_{H \mathbb{R}}(H \mathbb{R}[n], \theta(C)) .
$$

The vector space structure is induced by the monoidal structure on the homotopy categories and the fact that $\mathbb{R}$ and $H \mathbb{R}=\theta(\mathbb{R})$ are the units. For example, the action of $\mathbb{R}$ on $\pi_{*} E$ is given by

$$
\begin{aligned}
\operatorname{hMod}_{H \mathbb{R}}(H \mathbb{R}[n], E) \otimes \operatorname{hod}_{H \mathbb{R}}(H \mathbb{R}, H \mathbb{R}) & \rightarrow \operatorname{hMod}_{H \mathbb{R}}\left(H \mathbb{R}[n] \otimes_{H \mathbb{R}} H \mathbb{R}, E \otimes_{H \mathbb{R}} H \mathbb{R}\right) \\
& \cong \operatorname{hMod}_{H \mathbb{R}}(H \mathbb{R}[n], E),
\end{aligned}
$$


along with the isomorphism $\mathbb{R} \cong h \mathcal{D}\left(\operatorname{Vect}_{\mathbb{R}}\right)(\mathbb{R}, \mathbb{R}) \cong \operatorname{hMod}_{H \mathbb{R}}(H \mathbb{R}, H \mathbb{R})$, induced by $\mathrm{h} \theta$. This implies $\mathbb{R}$-linearity of $(94)$. To see the isomorphism $\mathbb{R} \cong \mathrm{hD}\left(\operatorname{Vect}_{\mathbb{R}}\right)(\mathbb{R}, \mathbb{R})$ recall that at the end of Example 9.3 we mentioned that the homotopy category of the unbounded derived category satisfies $\mathrm{h} \mathcal{D}\left(\operatorname{Vect}_{\mathbb{R}}\right)(C, D) \cong H_{0} \operatorname{Map}_{\mathbf{C h}_{\mathbb{R}}}(C, D)$. We must therefore compute all chain homotopy classes of chain maps $\mathbb{R}[0] \rightarrow \mathbb{R}[0]$. Clearly, any chain homotopy must be zero, so this is just $\operatorname{Hom}_{\mathbb{R}}(\mathbb{R}, \mathbb{R}) \cong \mathbb{R}$.

Suppose now that $E \in \operatorname{CAlg}\left(\operatorname{Mod}_{H \mathbb{R}}\right)$ and $C$ is a commutative DGA. Then $C$ may be regarded as an element of $\operatorname{CAlg}\left(\mathcal{D}\left(\operatorname{Vect}_{\mathbb{R}}\right)\right)$. In this case, $\pi_{*}(E)$ and $H_{*}(C)$ are graded commutative $\mathbb{R}$-algebras, induced by the following identifications and the symmetric monoidal structure on the homotopy categories $\mathrm{h} \mathcal{D}\left(\operatorname{Vect}_{\mathbb{R}}\right)$ and $\mathrm{hMod}_{H \mathbb{R}}$ :

$$
\begin{aligned}
& \mathbb{R}[n] \otimes \mathbb{R}[m] \cong \mathbb{R}[n+m] \\
& \theta \mathbb{R}[n] \otimes_{H \mathbb{R}} \theta \mathbb{R}[m] \cong \theta(\mathbb{R}[n] \otimes \mathbb{R}[m])=\theta \mathbb{R}[n+m] .
\end{aligned}
$$

It follows from this description that the maps (94) are the components of a map of graded algebras.

\subsubsection{Refinement for Modules}

We are now ready to generalize the first part of Dold's Theorem:

Theorem 9.9. Suppose $E \in \mathrm{Sp}, H \in \operatorname{Mod}_{H \mathbb{R}}$ and given a homomorphism of graded abelian groups

$$
\varphi: \pi_{*} E \rightarrow \pi_{*} H
$$

Then there exists a morphism $\phi: E \rightarrow H$ in $\mathrm{Sp}$, unique up to equivalence, with $\pi_{*} \phi=\varphi$.

Proof. Uniqueness: Suppose $\pi_{*} \phi_{1}=\pi_{*} \phi_{2}$ for $\phi_{1}, \phi_{2}: E \rightarrow H$ in Sp. By the essential surjectivity of $(93)$ we may find chain complexes $C, D$ with $\theta(C) \simeq E \wedge H \mathbb{R}$ and $\theta(D) \simeq H$. In fact, since over the reals any chain complex is quasi-isomorphic to a graded vector space with zero differential (the homology), we may even find graded vector spaces $V, W$ and equivalences

$$
\alpha: \theta(V) \stackrel{\simeq}{\rightarrow} E \wedge H \mathbb{R}, \quad \beta: \theta(W) \stackrel{\simeq}{\rightarrow} H .
$$


The forgetful functor $\operatorname{Mod}_{H \mathbb{R}} \rightarrow$ Sp has left adjoint $-\wedge H \mathbb{R}$. By Lur09, 5.2.2.8], this means that we have a weak equivalence

$$
\operatorname{Map}_{\operatorname{Mod}_{H \mathbb{R}}}(E \wedge H \mathbb{R}, H) \simeq \operatorname{Map}_{\mathrm{Sp}}(E, H) .
$$

It follows that we may find $\psi_{1}, \psi_{2}: E \wedge H \mathbb{R} \rightarrow H$ in $\operatorname{Mod}_{H \mathbb{R}}$ such that the homotopy class of $\psi_{1}$ is

$$
E \wedge H \mathbb{R} \stackrel{\left[\phi_{1}\right] \wedge H \mathbb{R}}{\longrightarrow} H \wedge H \mathbb{R} \rightarrow H,
$$

and similarly for $\phi_{2}$. In particular, $\pi_{*} \phi_{1}=\pi_{*} \phi_{2}$ implies that

$$
\pi_{*}\left(\psi_{1}\right)=\pi_{*}\left(\psi_{2}\right): \pi_{*}(E) \otimes \mathbb{R} \rightarrow \pi_{*}(H) \otimes \mathbb{R} \rightarrow \pi_{*}(H)
$$

coincide. Applying that $(93)$ is fully faithful to any choice of composition of

$$
\theta(V) \stackrel{\alpha}{\longrightarrow} E \wedge H \mathbb{R} \underset{\psi_{2}}{\stackrel{\psi_{1}}{\longrightarrow}} H \ll \beta
$$

we see that we may find $\varphi_{1}, \varphi_{2}: V \rightarrow W$ in $\mathcal{D}\left(\operatorname{Vect}_{\mathbb{R}}\right)$ such that the equivalence classes of $\theta\left(\varphi_{1}\right), \theta\left(\varphi_{2}\right)$ are $[\beta] \circ\left[\psi_{1}\right] \circ[\alpha]^{-1}$ and $[\beta] \circ\left[\psi_{2}\right] \circ[\alpha]^{-1}$, respectively. Consider the diagram

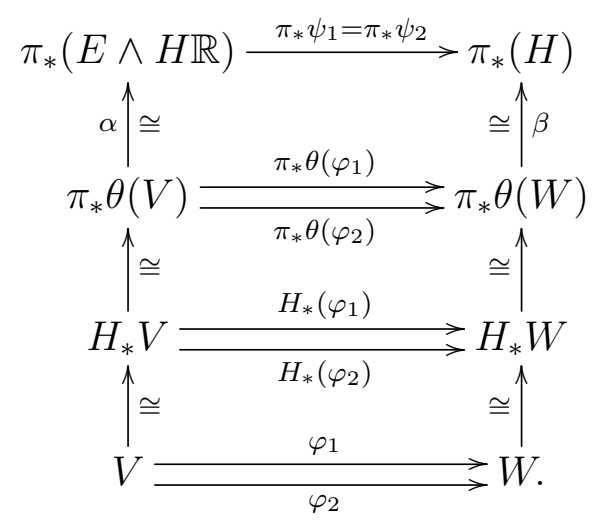

From the equality $\pi_{*} \psi_{1}=\pi_{*} \psi_{2}$ above and since the vertical maps are isomorphisms, we conclude $\varphi_{1}=\varphi_{2}$. Thus in $\operatorname{hMod}_{H \mathbb{R}}$ we have

$$
\left[\psi_{1}\right]=[\beta]^{-1} \circ\left[\theta\left(\varphi_{1}\right)\right] \circ[\alpha]=[\beta]^{-1} \circ\left[\theta\left(\varphi_{2}\right)\right] \circ[\alpha]=\left[\psi_{2}\right] .
$$

The equivalence 95 then implies $\phi_{1} \simeq \phi_{2}$. 
Existence: Choose $V, W$ and $\alpha, \beta$ as before. As in the diagram above, $V \cong \pi_{*}(E \wedge H \mathbb{R}) \cong \pi_{*}(E) \otimes \mathbb{R}$ and $W \cong \pi_{*}(H)$ so that $\varphi^{\text {adj }}: \pi_{*}(E) \otimes \mathbb{R} \rightarrow$ $\pi_{*}(H)$ gives rise to a homomorphism $\xi: V \rightarrow W$ as in

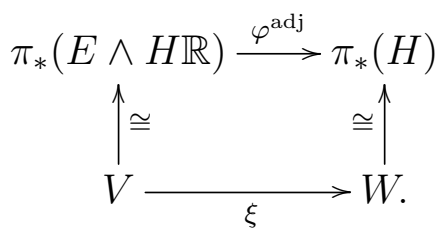

The required morphism $\phi$ is now any choice of composition in $\operatorname{Mod}_{H \mathbb{R}}$

$$
E \longrightarrow E \wedge H \mathbb{R} \stackrel{\alpha}{\longleftarrow} \theta(V) \stackrel{\theta(\xi)}{\longrightarrow} \theta(W) \stackrel{\beta}{\longrightarrow} H .
$$

On the level of homotopy groups, any such composition induces an adjoint to $\varphi$ and therefore coincides with $\varphi$.

The following subsection will be needed for our study of multiplicative structures. It addresses the behavior of various notions under equivalences.

\subsubsection{The Forgetful Functor on Algebras}

If $\mathscr{C}$ is a monoidal category, any category equivalent to $\mathscr{C}$ may also be endowed with a monoidal structure in such a way that the equivalence is a strong monoidal functor. The main result in this subsection (Proposition 9.10 may be regarded as a generalization of this statement.

For the reader uncomfortable with the techniques of $\infty$-categories I have formulated two immediate consequences (Corollaries 9.12 and 9.11) of Proposition 9.10 which are more easily accessible and play the main role in the sequel.

Let $p: \mathrm{e}^{\otimes} \rightarrow N \Gamma$ be a symmetric monoidal $\infty$-category with underlying $\infty$-category $\mathcal{C}=p^{-1}\langle 1\rangle$. Evaluation at $\langle 1\rangle \in \Gamma_{0}$ determines a simplicial map, the forgetful functor

$$
q: \mathrm{CAlg}(\mathrm{C}) \rightarrow \mathrm{C}
$$

The following technical proposition is proven using the language of marked simplicial sets, which is just a simplicial set $K$ along with a subset $s_{0} K_{0} \subset$ $\mathcal{E} \subset K_{1}$ of 'marked' edges [Lur09, 3.1]. Along with morphisms that preserve marked edges we obtain a category $\mathbf{S e t}_{\Delta}^{+}$. It carries a model structure 
(the Cartesian model structure) which is 'created' by marked anodyne maps (a class of monomorphisms with certain markings [Lur09, 3.1.1.1]) and the so-called Cartesian equivalences [Lur09, 3.1.3.7]. (The opposite of) marked simplicial sets are intimately tied to the homotopy theory (lifting arguments) of (co)Cartesian fibrations. There are three kinds of standard 'markings' on a simplicial set $K$ : $K^{\sharp}$ means all edges have been marked, $K^{b}$ means only degenerate edges have been marked, and $K^{\natural}$ makes sense only if there is understood an inner fibration $p: K \rightarrow L$ and then we mark the $p$-coCartesian edges. In this notation, a $p$-coCartesian fibration is then just a morphism $K^{\natural} \rightarrow L^{\sharp}$ which has the RLP for the opposite of all marked anodyne maps Lur09, 3.1.1.6].

Proposition 9.10. The map $q$ is a coCartesian fibration. The q-coCartesian edges $e \in \mathrm{CAlg}(\mathrm{C})_{1} \subset \operatorname{Set}_{\Delta}\left(N \Gamma \times \Delta^{1}, \mathrm{C}^{\otimes}\right)$ are characterized by the following condition: For every inert morphism $\sigma \in N \Gamma_{1}$ and every edge $\tau \in \Delta_{1}^{1}$ the image $e(\sigma, \tau)$ is p-coCartesian.

Proof. Let $I \subset N \Gamma_{1}$ be the set of inert morphisms in $\Gamma$, so that by definition $\operatorname{Fun}_{\operatorname{lax}}\left(N \Gamma, \mathrm{C}^{\otimes}\right)$ is the simplicial set underlying the marked simplicial set of sections

$$
\operatorname{Map}_{/ N \Gamma}\left((N \Gamma, I),\left(\mathcal{C}^{\otimes}\right)^{\natural}\right) .
$$

We will use the marking on $\mathrm{CAlg}(\mathcal{C})$ given by the internal hom of $\operatorname{Set}_{\Delta}^{+}$.

By the dual of [Lur09, 3.1.1.6] it will be sufficient to show that the marked simplicial map $q: \mathrm{CAlg}(\mathcal{C}) \rightarrow \mathcal{C}^{\sharp}$ has the right lifting property with respect to every marked morphism $i: A \rightarrow B$ opposite to a marked anodyne map. Let $j: \Delta^{0} \rightarrow N \Gamma$ denote the inclusion of the object $\langle 1\rangle$. Providing the dotted arrow in the diagram

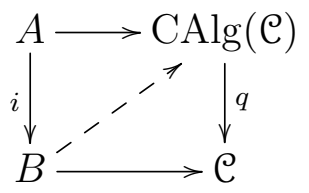

is equivalent to solving (the diagrams are adjoint to each other)

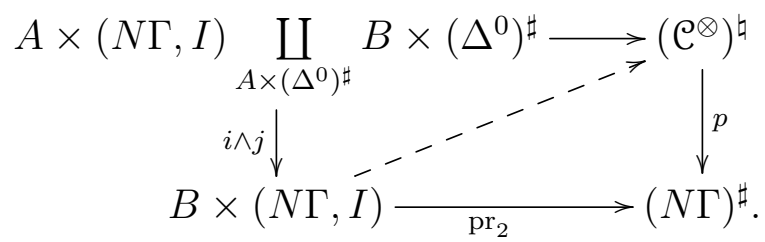


But by [Lur09, 3.1.2.3], the map $i^{\text {op }} \wedge j^{\text {op }}=(i \wedge j)^{\text {op }}$ is marked anodyne, so [Lur09, 3.1.1.6] implies that in the second diagram the dotted arrow exists.

Corollary 9.11. Let $A \in \mathrm{CAlg}(\mathcal{C})$ be an algebra object. Suppose the underlying object $q(A) \in \mathcal{C}$ is equivalent to some other object $B \in \mathcal{C}$. Then there exists an algebra object $B \in \mathrm{CAlg}(\mathcal{C})$ (with underlying object $B$ ) and an equivalence $A \simeq B$ in $\mathrm{CAlg}(\mathcal{C})$ covering the given equivalence.

Proof. Pick a $q$-coCartesian edge $e$ starting at $A$ and covering the equivalence $q(A) \rightarrow B$. By [Lur09, 2.4.1.5], $e$ is an equivalence in $\mathrm{CAlg}(\mathrm{C})$ and the target $d_{0} e$ is the required algebra object.

Corollary 9.12. Suppose $f: A \rightarrow B$ is a morphism in $\mathrm{CAlg}(\mathcal{C})$. Then any morphism $g: q(A) \rightarrow q(B)$ in $\mathcal{C}$ of the underlying objects that is equivalent to $q(f)$ may be endowed with the structure of a morphism of algebras: there exists $\hat{g}: A \rightarrow B$ in $\mathrm{CAlg}(\mathcal{C})$, equivalent to $f$, with $q(\hat{g})=g$.

Proof. Since $q(f)$ and $g$ are equivalent we may pick $\sigma: \Delta^{2} \rightarrow \mathcal{C}$ with $d_{2} \sigma=$ $q(f), d_{1} \sigma=g$ and so that $d_{0} \sigma=\mathrm{id}$, as depicted in the diagram

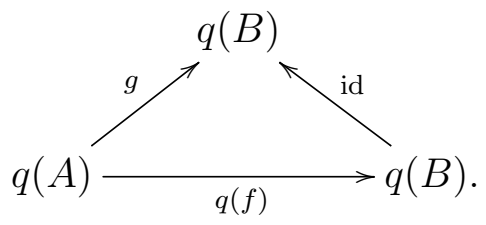

Since $\left(\Lambda_{1}^{2}\right)^{b} \subset\left(\Delta^{2}\right)^{b}$ (and its opposite) is marked anodyne and $\mathrm{CAlg}(\mathcal{C}) \rightarrow \mathcal{C}$ is a coCartesian fibration we may find $\hat{\sigma}: \Delta^{2} \rightarrow \mathrm{CAlg}(\mathcal{C})$ covering $\sigma$ with $d_{2} \hat{\sigma}=f, d_{0} \hat{\sigma}=\mathrm{id}$. The edge $\hat{g}=d_{1} \hat{\sigma}$ then has the required properties.

\subsubsection{Refinement for Algebras}

It is not surprising that we may not completely generalize the second part of Dold's Theorem because the homotopy/homology groups don't reflect the full homotopical characteristics of a product structure. For example, even though they split as chain complexes, only the so-called formal real DGAs are quasi-isomorphic to their homology. 
Since 93 is symmetric monoidal, it induces a corresponding functor between the categories of commutative algebra objects. Combining [Lur07b, Example 4.3.23] and [Lur07b, Theorem 4.3.22] with (93), we obtain an equivalence

$$
\theta: N\left(\mathbf{c D G A}^{\circ}\right) \stackrel{\simeq}{\rightarrow} \mathrm{CAlg}\left(\mathcal{D}\left(\operatorname{Vect}_{\mathbb{R}}\right)\right) \stackrel{\theta}{\rightarrow} \mathrm{CAlg}\left(\operatorname{Mod}_{H \mathbb{R}}\right) .
$$

This is also established in [Lur11, Theorem 8.1.4.11]. Note the restriction to the subcategory $\mathbf{c D G A}^{\circ}$ of cofibrant objects.

Proposition 9.13. Suppose $V, W$ are cofibrant graded commutative algebras and suppose given a morphism

$$
\phi: H V \rightarrow H W \text { in } \operatorname{Mod}_{H \mathbb{R}} .
$$

If $\pi_{*} \phi$ preserves the graded algebra structure, then we may refine $\phi$ to $\mathrm{CAlg}_{\mathbb{R}}$. This refinement is unique up to equivalence.

Proof. Existence: By (93) one may find a chain map $f: V \rightarrow W$ such that $\theta(f)$ is equivalent to $\phi$. In particular, we have a commutative diagram

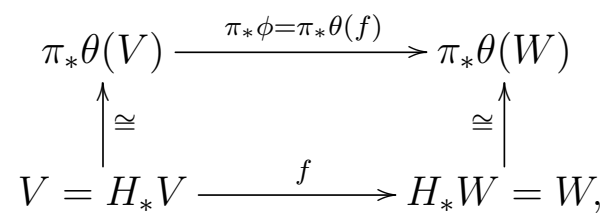

so that by assumption $f$ is a homomorphism of graded algebras. By (96) we may view $\theta(f)$ as a morphism in $\mathrm{CAlg}_{\mathbb{R}}$. Now apply Corollary 9.12 to $\phi \simeq \theta(f)$.

Uniqueness: Since (96) is fully faithful, any lift of $\phi$ is equivalent to the image under $\theta$ of a homomorphism $f: V \rightarrow W$ in $\mathbf{c D G A}^{\circ}$ which, using the last diagram, must correspond under the vertical isomorphisms to $\pi_{*} \phi$.

Remark 9.14. Suppose $V$ is a cofibrant graded algebra. Since the differential on $V$ is zero, it is generated as a graded commutative algebra by some set of generators of various degrees. Therefore, for any homomorphism $V \rightarrow H_{*} C$ of graded algebras (with $C \in \mathbf{c D G A}^{\circ}$ ) we may find some $V \rightarrow C$ in $\mathbf{c D G A}^{\circ}$ which induces $V \rightarrow H_{*} C$ in homology. Then the argument of the 
previous proposition shows the following:

(D) Suppose $H \in \mathrm{CAlg}_{\mathbb{R}}$. Under the above assumption, any morphism $\phi: H V \rightarrow H$ in $\operatorname{Mod}_{H \mathbb{R}}$ with $\pi_{*} \phi$ multiplicative may be refined to a morphism in $\mathrm{CAlg}_{\mathbb{R}}$.

We offer the following extension of the second part of Dold's Theorem:

Theorem 9.15. Let $V$ be cofibrant and let $E \in \mathrm{CAlg}_{\mathbb{R}}$. For any equivalence

$$
\phi: E \rightarrow H V \quad \text { in } \quad \operatorname{Mod}_{\mathbb{R}}
$$

such that $\pi_{*} \phi$ is a homomorphism of graded algebras there exists a unique up to equivalence refinement of $\phi$ to a morphism in $\mathrm{CAlg}_{\mathbb{R}}$.

Proof. Uniqueness: Given (necessarily equivalences) $\phi_{1}, \phi_{2}: E \rightarrow H V$ in $\mathrm{CAlg}_{\mathbb{R}}$ refining $\phi$, choose a composition $\psi$ as in

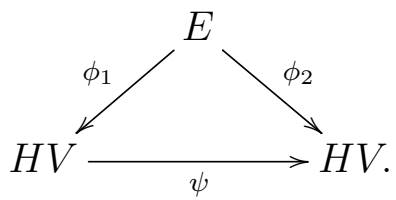

By (96) we may find a morphism $f: V \rightarrow V$ in $\mathbf{c D G A}^{\circ}$ such that $\theta(f)$ is equivalent in $\mathrm{CAlg}_{\mathbb{R}}$ to $\psi$. Therefore

$$
f=H_{*}(f)=\pi_{*} \theta(f)=\pi_{*} \psi=\pi_{*} \phi_{2} \circ \pi_{*} \phi_{1}^{-1}=\mathrm{id}
$$

so that $\psi \simeq \theta(f)$ is equivalent in $\mathrm{CAlg}_{\mathbb{R}}$ to id, that is, $\phi_{1} \simeq \phi_{2}$.

Existence: According to Corollary 9.11, there exists an object $H \in \mathrm{CAlg}_{\mathbb{R}}$ and an equivalence $\Phi: E \rightarrow H$ with lifting $\phi$. In particular, $H$ has underlying $H \mathbb{R}$-module $H V$. To find $\psi: H V \rightarrow H$ in $\mathrm{CAlg}_{\mathbb{R}}$ lifting the identity id ${ }_{H V}$ it suffices by $(\nabla)$ to show that the identity

$$
\pi_{*} H V \rightarrow \pi_{*} H
$$

is a homomorphism of graded algebras. Note here that $\pi_{*} H$ has the same underlying graded vector space than $\pi_{*} H V$, but a possibly different algebra 
structure. Now $(*)$ is multiplicative by the commutative diagram

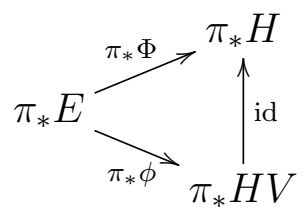

in which $\pi_{*} \Phi, \pi_{*} \phi$ are known to be isomorphisms of graded algebras. We obtain an equivalence

$$
\psi: H V \rightarrow H \quad \text { in } \quad \mathrm{CAlg}_{\mathbb{R}} \text { covering } \quad \mathrm{id}_{H V} .
$$

Any choice $\xi$ of composition of

$$
E \stackrel{\Phi}{\longrightarrow} H \stackrel{\psi}{\longleftarrow} H V
$$

satisfies $[q(\xi)] \simeq[q(\psi)]^{-1} \circ[q(\Phi)]=[\phi]$ in $\operatorname{hMod}_{H \mathbb{R}}$. By Corollary 9.12 we obtain the required lift of $\phi$ to $\mathrm{CAlg}_{\mathbb{R}}$.

\subsubsection{Refined Chern Character}

Suppose $E \in$ Sp. According to Theorem 9.9 from the canonical map $\pi_{*} E \rightarrow$ $V=\pi_{*} H V$ we obtain a morphism

$$
\phi: E \rightarrow H V \text { in } \mathrm{Sp},
$$

well-defined up to equivalence. Passing to the adjoint, this determines an equivalence class of morphisms in $\operatorname{Mod}_{H \mathbb{R}}$

$$
\phi^{\text {adj }}: E \wedge H \mathbb{R} \rightarrow H V .
$$

Assume that $V$ is cofibrant. By definition of the algebra structure on $V$, the map $\pi_{*} \phi^{\text {adj }}$ is a graded algebra isomorphism. Theorem 9.15 determines a lift

$$
E \wedge H \mathbb{R} \rightarrow H V \text { in } \quad \mathrm{CAlg}_{\mathbb{R}}
$$

well-defined up to equivalence. Precomposing with $E \rightarrow E \wedge H \mathbb{R}$ now singles out a canonical equivalence class of a morphism ch : $E \rightarrow H V$ in $\mathrm{CAlg}(\mathrm{Sp})$, which we call the refined Chern character. This is our canonical multiplicative datum.

Example 9.16. We list some cohomology theories $E$ whose coefficient algebra $V=\pi_{*} E \otimes \mathbb{R}$ satisfies the hypothesis of $V$ being cofibrant: $k u$ (connective $K$-theory), $M U, M O, \mathbb{S}$ (sphere spectrum), $H V$. 


\section{UniquenEss (GENERAL CASE)}

As mentioned in Subsection 1.2.2, for rationally even cohomology theories there is an essentially unique differential refinement of a generalized cohomology theory [BS10, Theorem 3.10], at least if we demand compatibility with integration maps.

In this chapter, we shall investigate how to answer the corresponding question without making further assumptions. We will prove that the axioms from Definition 1.12 determine the differential extension up to 'equivalence,' a weakening of the notion of 'isomorphic differential extensions' from [BS10].

Definition 10.1. Let $(\hat{E}, I, R, a)$ be a differential extension of $E$. For each $n \in \mathbb{Z}$ and $M \in$ Man the transformation $a: \Omega^{n-1}(M ; V)_{d} \rightarrow \hat{E}^{n}(M)$ gives rise to a (standard) strict symmetric monoidal groupoid

$$
\hat{\mathscr{E}}^{n}(M)=\left\{\begin{array}{l}
\operatorname{objects:} x, y, z, \ldots \in \hat{E}^{n}(M) \\
\operatorname{Hom}_{\hat{\mathscr{E}}(M)}(x, y)=\left\{\eta \in \Omega^{n-1}(M ; V)_{d} \mid a(\eta)=y-x\right\} \\
\operatorname{composition~\& ~monoidal~structure:~}+^{+}
\end{array}\right.
$$

For varying $M$, these may be organized into a functor

$$
\hat{\mathscr{E}}^{n}: \text { Man } \rightarrow \text { BrMonCat }_{\text {strict }} .
$$

In the same way, the differentials $d: \Omega^{n-1}(M ; V)_{d} \rightarrow \Omega^{n}(M ; V)$ and $\delta: C^{n-1}(M ; V)_{\delta} \rightarrow C^{n}(M ; V)$ give functors

$$
\mathscr{O}^{n}, \mathscr{C}^{n}: \operatorname{Man} \rightarrow \text { BrMonCat }_{\text {strict }}
$$

(by Example A.3). The transformation $R$ along with the property $R a=d$ amounts to a natural transformation $R: \hat{\mathscr{E}}^{n} \rightarrow \mathscr{O}^{n}$.

Definition 10.2. An equivalence between differential extensions $\hat{E}_{1}, \hat{E}_{2}$ of $E$ is a natural equivalence of the corresponding symmetric monoidal groupoids

$$
\hat{\mathscr{E}}_{1} n(M) \stackrel{\simeq}{\longrightarrow} \hat{\mathscr{E}}_{2} n(M), \quad \forall n \in \mathbb{Z} .
$$

Theorem 10.3. Any two differential extensions of $E$ are equivalent. 
Proof. Suppose $\hat{E}_{1}, \hat{E}_{2}$ are differential extensions of $E$ and form the corresponding functors $\hat{\mathscr{E}}_{1}, \hat{\mathscr{E}}_{2}$. We will construct a natural equivalence of symmetric monoidal groupoids

$$
\Phi_{M}: \hat{\mathscr{E}}_{1}^{n}(M) \longrightarrow \hat{\mathscr{E}}_{2}^{n}(M), \quad n \in \mathbb{Z}
$$

We will use the results from [BS10, Sections 2 and 3], whose notation we now quickly recall: represent $E^{*}$ by an $\Omega$-spectrum $\left(E_{n}, \varepsilon_{n}\right)$. Fix a degree $n$. According to BS10, Proposition 2.3] there exists a sequence of pointed manifolds $\varepsilon_{n}^{(i)}, i \in \mathbb{N}$, together with pointed embeddings of submanifolds $\kappa_{i}: \mathcal{E}_{n}^{(i)} \hookrightarrow \mathcal{E}_{n}^{(i+1)}$ as well as $(i-1)$-connected pointed maps $x_{i}: \varepsilon_{n}^{(i)} \rightarrow E_{n}$ such that $x_{i+1} \circ \kappa_{i}=x_{i}$ and such that for any finite-dimensional pointed CW-complex $X$ the canonical map

$$
\operatorname{colim}_{i}\left[X, \mathcal{E}_{n}^{(i)}\right] \rightarrow\left[X, E_{n}\right]=E^{n}(X)
$$

is an isomorphism. Using [BS10, Propositions 2.5, 2.6] one may choose $\hat{u}_{i} \in$ $\hat{E}_{1}^{n}\left(\mathcal{E}_{n}^{(i)}\right)$ with

$$
\kappa_{i}^{*} \hat{u}_{i+1}=\hat{u}_{i}, \quad I\left(\hat{u}_{i}\right)=\left[x_{i}\right], \quad \operatorname{Rham}\left(R\left(\hat{u}_{i}\right)\right)=\operatorname{ch}\left[x_{i}\right] .
$$

In the same way one may choose elements $\hat{u}_{i}^{\prime} \in \hat{E}_{2}^{n}\left(\mathcal{E}_{n}^{(i)}\right)$. We are now ready to recall the definition of $\Phi_{M}$ as a set map $\hat{E}_{1}^{n}(M) \rightarrow \hat{E}_{2}^{n}(M)$ : For $\hat{v} \in \hat{E}_{1}^{n}(M)$ write $I(\hat{v})=\left[M \stackrel{f}{\rightarrow} E_{n}\right]$ for some $f$ and factor $f \simeq x_{i} \circ f_{i}$ up to homotopy for some $f_{i}: M \rightarrow \mathcal{E}_{n}^{(i)}$. Then, by the exact sequence defining differential extensions (Definition 1.12),

$$
\hat{v}-f_{i}^{*} \hat{u}_{i}=a(\alpha) \text { for some } \alpha \in \Omega^{n-1}(M ; V)_{d},
$$

and we define

$$
\Phi_{M}(\hat{v}):=f_{i}^{*}\left(\hat{u}_{i}^{\prime}\right)+a(\alpha) .
$$

As shown in [BS10, Lemmas 3.2, 3.3, 3.4], $\Phi_{M}$ is well-defined (independent of the choice of $\alpha$ and $f_{i}$ ), compatible with $I, R, a$ and natural in $M$. We use the identity on $\Omega^{n-1}(M ; V)_{d}$ to view $\Phi_{M}$ as a functor

$$
\Phi_{M}: \hat{\mathscr{E}}_{1}^{n}(M) \rightarrow \hat{\mathscr{E}}_{2} n(M)
$$

which is of course fully faithful. It is also essentially surjective: For $\hat{u}^{\prime} \in$ $\hat{E}_{2}^{*}(M)$ choose $\hat{u} \in \hat{E}_{1}^{*}(M)$ with $I(\hat{u})=I^{\prime}\left(\hat{u}^{\prime}\right)$. Using that $I \circ \Phi_{M}=I$ and 
again the exact sequence, we may write $\Phi_{M}(\hat{u})-\hat{u}^{\prime}=a(\alpha) \in \hat{E}_{2}^{n}(M)$ for some $\alpha \in \Omega^{n-1}(M ; V)_{d}$. Thus, using $\Phi_{M} \circ a=a$, the object $\hat{u}-a(\alpha) \in \hat{\mathscr{E}}_{1}(M)$ clearly maps to $\hat{u}^{\prime}$.

It follows that $\Phi_{M}$ is an equivalence of categories. It remains therefore to show that $\Phi_{M}$ is a symmetric monoidal functor. The deviation of $\Phi_{M}$ from being additive is a natural transformation

$$
\left(\Phi_{M} \circ+\right)-\left(+^{\prime} \circ\left(\Phi_{M} \times \Phi_{M}\right)\right): \hat{E}_{1}^{n}(M) \times \hat{E}_{1}^{n}(M) \rightarrow \hat{E}_{2}^{n}(M)
$$

that composes with $I$ to zero, so that it factors over a natural transformation

$$
B: \hat{E}_{1}^{n}(M) \times \hat{E}_{1}^{n}(M) \rightarrow \Omega^{n-1}(M ; V)_{d} / \mathrm{im}(\mathrm{ch}) .
$$

satisfying

$$
\begin{aligned}
& B(\hat{u}, \hat{v}+\hat{w})+B(\hat{v}, \hat{w})=B(\hat{u}, \hat{v})+B(\hat{u}+\hat{v}, \hat{w}), \\
& B(\hat{u}, \hat{v})=B(\hat{v}, \hat{u}), \\
& B(\hat{u}, 0)=0=B(0, \hat{u}) .
\end{aligned}
$$

Together with the identity arrows $0 \stackrel{0}{\rightarrow} \Phi_{M}(0)=0$ the transformation $B$ gives $\Phi_{M}$ the structure of a symmetric monoidal functor. The naturality of $B$ in the objects of $\hat{\mathscr{E}}_{1}^{n}(M)$ follows from the observation that

$$
\Phi_{M}(\hat{v}+a(\beta))=\Phi_{M}(\hat{v})+a(\beta) .
$$

Remark 10.4. Under additional assumptions (rationally even, finiteness conditions on the coefficients) the results of [BS10] amount to the assertion that one may even pick a strict monoidal equivalence.

\section{FiELD THEORY INTERPRETATION}

On closed, differentially oriented $n$-manifolds $M$, differential cohomology classes $\hat{x} \in \hat{H}^{n+1}(M)$ may be integrated to numbers

$$
\int_{M} \hat{x} \in \mathbb{R} / \mathbb{Z}=S^{1} \subset \mathbb{C} .
$$


An example of this construction is the Chern-Simons action

$$
S(\hat{x})=\int_{M} \hat{x} \cup \hat{x}
$$

of a principal $S^{1}$-bundle $P \rightarrow M$ with connection $\nabla$ on a 3-manifold $M$ (represented by $\hat{x} \in \hat{H}^{2}(M)$ ). By (85), the expression (99) agrees with the more traditional formula

$$
S(A)=\int_{M} A \wedge d A+\frac{2}{3} A \wedge A \wedge A, \quad A \in \Omega^{1}(M)
$$

for topologically trivial bundles $\hat{x}=a(A)$ with connection $A$.

For bundles $P$ that are trivial (but not trivialized) one needs to choose a section of $P$ to define the action $S(P, \nabla)$. If $M$ is closed, an application of Stokes' Theorem shows that $S$ is well-defined up to integers. However, if $M$ has boundary, this is no longer true. The remarkable solution to this problem is to interpret the action as an element of an abstract complex line which belongs to the restriction of the field $(P, \nabla)$ to the boundary: these are the Wess-Zumino-Witten Lines $\left.S(P, \nabla)\right|_{\partial M}$. The action determines a well-defined element $S(P) \in S(P \mid \partial M)$ in this line.

This may be summarized by saying that $S$ is the action of a Lagrangian field theory [Fre95], which means the following:

- We have groupoids of 'fields' $\mathfrak{C}_{M}, \mathfrak{C}_{N}$ for every 3-manifold $M$ (possibly with boundary) and every 2-manifold $N$ (we have taken the groupoids of principal circle bundles with connection above).

- There is given an action $S$ which assigns complex lines to fields on 2manifolds. Fields $\phi$ on 3-manifolds with boundary $M$ have an action $S(\phi)$ which is an element of the line $S(\phi \mid \partial M)$.

These are required to satisfy various functoriality and locality properties (see [Fre95, Theorem 2.19]).

The integral (98) is not well-defined unless $\partial M=\emptyset$. In this section, we will present a generalization of the WZW-construction for the solution 
of this problem (Theorem 11.15). This will be carried out for ordinary differential cohomology (which can be handled using ordinary category theory).

Using the product structure developed in this thesis, the differential intersection pairing $S(\hat{x})=\int_{M} \hat{x} \cup \hat{x}$ can be studied also for generalized cohomology theories $E$. These generalizations of the Chern-Simons action probably also belong to certain Lagrangian field theories, but the corresponding integral (98) for manifolds with boundary has not yet been developed (and probably requires the use of higher categories of fields).

In the final section of this thesis, we show that in the case of $K$-theory and $\partial M=\emptyset$ this intersection pairing is non-trivial. It can be identified (up to rationals) with a certain $\eta$-invariant.

\section{$11.1 \quad$ Fields}

Ordinary differential cohomology $\hat{H}^{n}(M)=H^{n}\left(\hat{C}(n)^{*}(M)\right)$ may be defined in terms of cochain complexes [HS05, Definition 2.5]:

\section{Definition 11.1.}

$$
\hat{C}(n)^{*}(M)= \begin{cases}C^{*}(M ; \mathbb{Z}) \times C^{*-1}(M ; \mathbb{R}) \times \Omega^{*}(M) & (* \geq n), \\ C^{*}(M ; \mathbb{Z}) \times C^{*-1}(M ; \mathbb{R}) \times\{0\} & (*<n),\end{cases}
$$

with differential

$$
d(c, h, \omega)=(\delta c, \omega-c-\delta h, d \omega)
$$

Definition 11.2. Let $\hat{\mathcal{H}}^{n}(M)$ be the monoidal groupoids with $\pi_{0} \hat{\mathcal{H}}^{n}(M)=$ $\hat{H}^{n}(M)$ obtained by from $\hat{C}(n)^{*}$ at level $n$ by Example A.3.

The objects of $\hat{\mathcal{H}}^{n}(M)$ are the cocycles $x \in \hat{C}(n)^{n}(M), d x=0$. The morphisms $x_{1} \rightarrow x_{2}$ are $\operatorname{im}(d)$-cosets of cochains $y \in \hat{C}(n)^{n-1}(M)$ with $d y=x_{1}-x_{2}$.

Recall the natural chain homotopy $B$ from Example 7.11. The cupproduct in differential cohomology is a chain map (using (72)

$$
\cup: \hat{C}(n)^{*}(M) \otimes \hat{C}(m)^{*}(M) \rightarrow \hat{C}(n+m)^{*}(M),
$$


defined by the formula

$\left(c_{0}, h_{0}, \omega_{0}\right) \cup\left(c_{1}, h_{1}, \omega_{1}\right)=\left(c_{0} \cup c_{1},(-1)^{n} c_{0} \cup h_{1}+h_{0} \cup \omega_{1}+B\left(\omega_{0}, \omega_{1}\right), \omega_{0} \wedge \omega_{1}\right)$.

This chain map gives rise to bilinear functors [HS05, p.13]

$$
\cup: \hat{\mathcal{H}}^{n}(M) \times \hat{\mathcal{H}}^{m}(M) \rightarrow \hat{\mathcal{H}}^{n+m}(M) .
$$

For $n=0,1,2$ these are familiar geometric categories:

Example 11.3. $\hat{\mathcal{H}}^{0}(M) \cong C^{\infty}(M, \mathbb{Z})$ (discrete category).

Example 11.4. $\hat{\mathcal{H}}^{1}(M)$ is the category of (set) maps $f: M \rightarrow \mathbb{R}$ that are smooth when post-composed to $\mathbb{R} / \mathbb{Z}$; there is a unique morphism $f_{1} \rightarrow f_{2}$ whenever $f_{1}-f_{2}$ is integer-valued.

Example 11.5. $\hat{\mathcal{H}}^{2}(M)$ is equivalent to the category of circle bundles with connection [HS05, Example 2.7]. This is a monoidal equivalence if we take the monoidal structure $P \times_{S^{1}} Q$ on principal bundles? Writing down the equivalence in [HS05, Example 2.7] in the special case $M=\mathrm{pt}$, one sees that $\hat{\mathcal{H}}^{2}(\mathrm{pt}) \simeq \mathbb{R} / \mathbb{Z}$-Spaces sends every object to $\mathbb{I}=\mathbb{R} / \mathbb{Z}$. The category $\mathbb{R} / \mathbb{Z}$-Spaces is equivalent to the category HermLines of Hermitian lines (one-dimensional complex vector spaces with Hermitian metric).

\subsection{Push-Forward Maps}

Let $p: E \rightarrow S$ be a smooth map between compact manifolds $E, S$. The relative dimension of $p$ is $k=\operatorname{dim} E-\operatorname{dim} S$. Analogous to the case of generalized cohomology theories, [HS05, Section 2.4] define push-forward maps in differential cohomology. We will recall their construction in this section.

Definition 11.6. A differential orientation $(j, W, J, U)$ on $p$ consists of the following data:

1. An embedding $j: E \hookrightarrow S \times \mathbb{S}^{N}$ with $\operatorname{pr}_{1} \circ j=p$ and a tubular neighborhood of $j$ (that is, a vector bundle $\pi: W \rightarrow E$ and an extension of $j$ to an open embedding $J: W \hookrightarrow S \times \mathbb{S}^{N}$ with $\left.\operatorname{pr}_{1} \circ J=p \circ \pi\right)$.

\footnotetext{
${ }^{9} P \times{ }_{S^{1}} Q$ is $P \times Q$ modulo the diagonal action $(p . z, q) \sim(p, z . q), z \in S^{1}$.
} 
Figure 1: The data of 1 . is called a framing of $p: E \rightarrow S$.

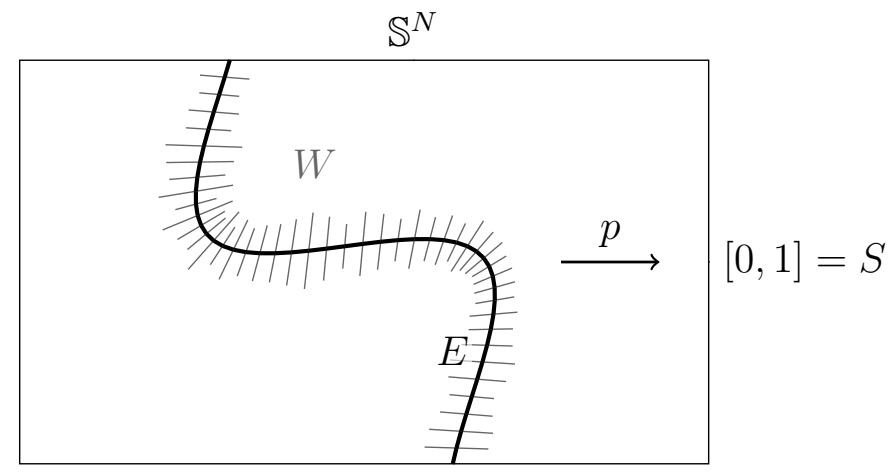

2. A differential 'Thom' cocycle $U=(c, h, \omega) \in \hat{Z}(N-k)_{\mathrm{c}}^{N-k}(W)$ which is compactly supported ${ }^{10}$. It is required that $\omega \in \Omega^{N-k}(W)$ define an ordinary orientation 11 of $W$.

A quadruple $(j, W, J, \hat{U})$ as above with $\hat{U} \in \hat{H}_{\mathrm{c}}^{N-k}(W)(R(\hat{U})$ determining an orientation on $W$ ) will be called a differential orientation class.

The slant product of cochains and the integral of forms along the fiber $\mathbb{S}^{N}$ determines a cochain map (using Lemma 6.2 (ii))

$$
\int_{\mathbb{S}^{N}}: \hat{C}(n+N)^{*}\left(S \times \mathbb{S}^{N}\right) \rightarrow \hat{C}(n)^{*}(S) .
$$

Explicitly, $\int_{\mathbb{S}^{N}}(c, h, \omega)=\left(\int_{\mathbb{S}^{N}} c, \int_{\mathbb{S}^{N}} h, \int_{\mathbb{S}^{N}} \omega\right)$.

Definition 11.7. Given a differential orientation on $p$, we get a cochain map, the push-forward along $p$, denoted either by $p_{!}, \int_{E / S}$, or $\int^{U}$ :

$$
\begin{aligned}
\hat{C}(m+k)^{*+k}(E) \stackrel{\pi^{*}}{\longrightarrow} \hat{C}(m+k)^{*+k}(W) \stackrel{-\cup U}{\longrightarrow} \hat{C}(m+N)_{\mathrm{c}}^{*+N}(W) \\
\stackrel{J_{*}}{\rightarrow} \hat{C}(m+N)^{*+N}\left(S \times \mathbb{S}^{N}\right) \stackrel{\int_{\mathbb{S}^{N}}}{\longrightarrow} \hat{C}(m)^{*}(S) .
\end{aligned}
$$

\footnotetext{
${ }^{10}$ There exists $K \subset W$ compact so that the restriction of $U$ to $W \backslash K$ vanishes

${ }^{11}$ This means that $\omega\left(v_{1}, \ldots, v_{N-k}\right) \neq 0$ for any basis $v_{1}, \ldots, v_{N-k} \in W_{e} \subset T_{e} E, e \in E$.
} 
That is,

$$
\int^{U} x=\int_{\mathbb{S}^{N}} J_{*}\left(\pi^{*} x \cup U\right)
$$

\subsection{Integration on Closed Manifolds}

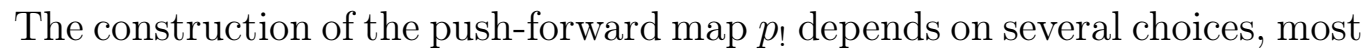
notably a framing and a differential Thom cocycle $U$. The framing is a feature of the situation (in Chern-Simons theory certain numbers are known to depend on the choice of framing). We will see however that, interpreted suitably, the integral depends only on the differential class $\hat{U}=[U] \in \hat{H}_{\mathrm{c}}^{N-k}(W)$.

Fix a smooth map $p: E \rightarrow S$ between compact manifolds and a framing $J: W \hookrightarrow S \times \mathbb{S}^{N}$ on $p$. Given $\hat{U} \in \hat{H}_{\mathrm{c}}^{N-k}(W)$, let

$$
\mathscr{U}= \begin{cases}\text { objects: } & U \in \hat{Z}(N-k)_{\mathrm{c}}^{N-k}(W) \text { with } \hat{U}=[U], \\ \text { morph. } U_{1} \rightarrow U_{2}: & V \in \hat{C}(N-k)_{\mathrm{c}}^{N-k-1}(W) / \operatorname{im}(d) \text { with } d V=U_{1}-U_{2} .\end{cases}
$$

This category is connected (meaning there is an arrow between any two objects). We begin by constructing a functor

$$
\Psi: \hat{\mathcal{H}}^{n+k}(E) \rightarrow \operatorname{Fun}\left(\mathscr{U}, \hat{\mathcal{H}}^{n}(S)\right)
$$

or, more precisely, its adjoint $F: \hat{\mathcal{H}}^{n+k}(E) \times \mathscr{U} \rightarrow \hat{\mathcal{H}}^{n}(S)$. On objects $x \in \hat{Z}(n+k)^{n+k}(E), U \in \hat{Z}(N-k)_{\mathrm{c}}^{N-k}(W)$, the functor $F$ is given by

$$
F(x, U)=\int^{U} x \in \hat{Z}(n)^{n}(S) .
$$

To a morphism $y \in \hat{C}(n+k)^{n+k-1}(E) / \operatorname{im}(d), V \in \hat{C}^{N-k-1}(N-k)_{\mathrm{c}}(W) / \operatorname{im}(d)$ from $\left(x_{1}, U_{1}\right)$ to $\left(x_{2}, U_{2}\right)$ the functor $F$ assigns

$$
F(y, V)=\int_{\mathbb{S}^{N}} J_{*}\left(\pi^{*} y \cup U_{1}\right)+J_{*}\left(\pi^{*} x_{2} \cup V\right) .
$$

Since 100, 101 are cochain maps, this is indeed a morphisms from $\int^{U_{1}} x_{1}$ to $\int^{U_{2}} x_{2}$. Linearity implies the functoriality of $F$.

Lemma 11.8. The functor 103 maps into the subcategory of 'holonomyfree' functors: for each $x \in \hat{\mathcal{H}}^{n+k}(E)$ the functor $F(x,-)$ takes automorphisms to the identity. 
Proof. Suppose $V \in \hat{C}(N-k)_{\mathrm{c}}^{N-k-1}(W)$ represents an automorphism, $d V=$ 0 . By [HS05, p. 25], the $(N-k-1)$-th cohomology of $\hat{C}(N-k)_{\mathrm{c}}^{*}(W)$ can be identified with $H_{\mathrm{c}}^{N-k-2}(W ; \mathbb{R}) / H_{\mathrm{c}}^{N-k-2}(W ; \mathbb{Z})$. By the Thom isomorphism, this is just $H_{\mathrm{c}}^{-2}(W ; \mathbb{R}) / H_{\mathrm{c}}^{-2}(W ; \mathbb{Z})$, which is zero. We may therefore find $L \in \hat{C}(N-k)_{\mathrm{c}}^{N-k-2}(W)$ with $d L=V$. Since (100), (101) are cochain maps,

$$
F\left(\operatorname{id}_{x}, V\right)=\int_{\mathbb{S}^{N}} J_{*}\left(\pi^{*} x \cup V\right)= \pm d \int_{\mathbb{S}^{N}} J_{*}\left(\pi^{*} x \cup L\right) \pm \int_{\mathbb{S}^{N}} J_{*}\left(\pi^{*} d x \cup L\right) .
$$

Since $x$ is closed, $F\left(\mathrm{id}_{x}, V\right)$ bounds (so represents the identity).

A diagram $F: I \rightarrow \mathcal{C}$ into a groupoid $\mathcal{C}$ (with $I$ connected) has a limit precisely when $F$ is holonomy-free. Composing (103) with the limit

$$
\operatorname{Fun}_{\text {hol-free }}\left(\mathscr{U}, \hat{\mathcal{H}}^{n}(S)\right) \stackrel{\lim }{\longrightarrow} \hat{\mathcal{H}}^{n}(S)
$$

determines a functor that depends only upon the class $\hat{U} \in \hat{H}_{\mathrm{c}}^{N-k}(W)$ :

$$
\hat{\mathcal{H}}^{n+k}(E) \rightarrow \hat{\mathcal{H}}^{n}(S)
$$

We summarize our discussion as follows:

Proposition 11.9. The exists a canonical functor (104), depending only on the differential orientation class $\hat{U}$ of $p$, which refines the push-forward $p_{!}: \hat{H}^{n+k}(E) \rightarrow \hat{H}^{n}(S)$ in differential cohomology (by passing to connected components).

Remark 11.10. Restricting to the 'flat' subcategory $\hat{\mathcal{H}}_{\text {flat }}^{n+k}(E)$, one may consider the dependence of this functor on $\hat{U} \rightarrow \hat{U}+a(\theta)$. More precisely, given an ordinary Thom class $U \in H_{\mathrm{c}}^{N-k}(W)$ define $\mathrm{Ob}(\mathscr{I}(U))=I^{-1}(U)$, a morphism $U_{1} \rightarrow U_{2}$ being a $\operatorname{im}(d)$-coset of differential forms $\theta \in \Omega^{N-k-1}(W)$ with $a(\theta)=U_{1}-U_{2}$. For $x$ flat, $\hat{U} \mapsto \int^{\hat{U}} x$ turns out to be holonomy-free as well (by the Thom isomorphism), so one obtains a functor

$$
\hat{\mathcal{H}}_{\text {flat }}^{n+k}(E) \rightarrow \hat{\mathcal{H}}^{n}(S)
$$

that depends only on the ordinary orientation of the normal bundle $W$. 
Definition 11.11. A differential orientation on a closed $n$-manifold $N$ is defined to be a differential orientation class $(j, W, J, \hat{U})$ of the projection $p: N \rightarrow$ pt. Using the identification of Example 11.5, we define a functor

$$
S^{\hat{U}}: \hat{\mathcal{H}}^{n+2}(N) \rightarrow \text { HermLines }
$$

as the post-composition with 'lim' of the functor

$$
\Psi: \hat{\mathcal{H}}^{n+2}(N) \stackrel{\text { 103) }}{\longrightarrow} \text { Fun }_{\text {hol-free }}\left(\mathscr{U}, \hat{\mathcal{H}}^{2}(p t)\right) \stackrel{\simeq}{\longrightarrow} \text { Fun }_{\text {hol-free }}(\mathscr{U}, \text { HermLines }) .
$$

We will keep writing $\Psi(x)(U)=\int^{U} x$ on objects, now viewed as an object of HermLines. For each morphism $V$ in $\mathscr{U}, \Psi(x)\left(U_{1} \stackrel{V}{\rightarrow} U_{2}\right)$ is a Hermitian isomorphism (given by multiplication with $\left.\int_{\mathbb{S}^{N}} J_{*}\left(\pi^{*} x \cup V\right) \in S^{1}\right)$. Recall that the equivalence $\hat{\mathcal{H}}^{2}(p t) \simeq \mathbb{R} / \mathbb{Z}$-Spaces $\simeq$ HermLines in Example 11.5 takes every object to the identity object $\mathbb{C}$. Therefore,

$$
\int^{U} x=\mathbb{C}, \quad \forall x \in \operatorname{Ob}\left(\hat{\mathcal{H}}^{n+2}(N)\right),
$$

for every differential cocycle $U$ representing $\hat{U}$. By definition of the limit in a groupoid, we have an isomorphism $\phi_{U}: S^{\hat{U}}(x) \stackrel{\cong}{\rightarrow} \int^{U} x$. Also, $\phi_{U_{1}}=$ $\Psi(x)\left(U_{1} \stackrel{V}{\rightarrow} U_{2}\right) \circ \phi_{U_{2}}$. In other words:

Proposition 11.12. Let $N$ be a closed, differentially oriented n-manifold. To every object $x \in \hat{\mathcal{H}}^{n+2}(N)$ we may functorially associate a Hermitian line $S^{\hat{U}}(x)$. Any choice of cocycle $U$ representing $\hat{U}$ determines a trivialization $\phi_{U}$ of $S^{\hat{U}}(x)$. If $d V=U_{1}-U_{2}$ are two choices of trivialization, $\Psi(x)(V)$ is a Hermitian isomorphism $\mathbb{C}=\int^{U_{1}} x \rightarrow \int^{U_{2}} x=\mathbb{C}$, making the following diagram commute:

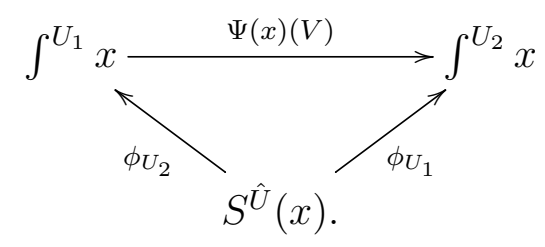




\subsection{Manifolds with Boundary}

Definition 11.13. Let $M$ be a compact $(n+1)$-manifold, equipped with a map $f: M \rightarrow[0,1]$ which defines the boundary. A differential orientation on $M$ is a differential orientation class $(j, W, J, \hat{U})$ on $f$.

For each cocycle representative $U$ of $\hat{U}$ we define

$$
\int_{M / \mathrm{pt}}^{U}: \hat{C}(m+n+1)^{*+n+1}(M) \stackrel{f_{1}}{\rightarrow} \hat{C}(m+1)^{*+1}([0,1]) \stackrel{\int_{0}^{1}}{\rightarrow} \hat{C}(m)^{*}(\mathrm{pt}) .
$$

This is not a chain map, so there is no induced map in differential cohomology. Instead, one has a version of Stokes' Theorem [HS05, (2.10)]

$$
d \int_{M / \mathrm{pt}}^{U} x=\int_{M / \mathrm{pt}}^{U} d x-(-1)^{|x|} \int_{\partial M / \mathrm{pt}}^{U \mid \partial M} x .
$$

We have $\hat{C}(1)^{1}(\mathrm{pt})=\mathbb{Z} \times \mathbb{R}$, which we interpret as a number via $\mathbb{Z} \times \mathbb{R} \rightarrow$ $\mathbb{R} / \mathbb{Z} \subset \mathbb{C}$.

Definition 11.14. Suppose $\hat{U} \in \hat{H}^{N-n-1}(W)$ is a differential Thom class on $M$. For $x \in \hat{\mathcal{H}}^{n+2}(M)$ we define $S^{\hat{U}}(x)$ as the following element of $S^{\hat{U} \mid \partial M}\left(\left.x\right|_{\partial M}\right)$ : for every cocycle $U$ representing $\hat{U}$, let $S^{\hat{U}}(x)$ denote the preimage of $\int_{M / \mathrm{pt}}^{U} x \in \mathbb{C}$ under the isomorphism $\phi_{U \mid \partial M}: S^{\hat{U} \mid \partial M}\left(\left.x\right|_{\partial M}\right) \rightarrow \int^{U \mid \partial M} x=$ $\mathbb{C}$.

It remains to check that this is well-defined. Suppose $U_{1}, U_{2}$ are two trivializations of $\hat{U}$ and pick $V$ with $d V=U_{1}-U_{2}$. We claim that $\phi_{U_{1} \mid \partial M}^{-1}\left(\int_{M / \mathrm{pt}}^{U_{1}} x\right)=$ $\phi_{U_{2} \mid \partial M}^{-1}\left(\int_{M / \mathrm{pt}}^{U_{2}} x\right)$. By Proposition 11.12 , the right-hand side equals $\Psi(x)(V \mid \partial M) \circ$ $\phi_{U_{1} \mid \partial M}^{-1}\left(\int_{M / \mathrm{pt}}^{U_{2}} x\right)$, so we are claiming

$$
\int_{M / \mathrm{pt}}^{U_{1}} x=\int_{\mathbb{S}^{N}} J_{*}\left(\left.\left.\pi^{*} x\right|_{\partial M} \cup V\right|_{\partial M}\right)+\int_{M / \mathrm{pt}}^{U_{2}} x=\int_{\partial M / \mathrm{pt}}^{V} x+\int_{M / \mathrm{pt}}^{U_{2}} x .
$$

This follows from (106). 
Theorem 11.15. For every differentially oriented $n$-manifold $N$, we have a functor

$$
S^{\hat{U}}: \hat{\mathcal{H}}^{n+2}(N) \rightarrow \text { HermLines }
$$

that depends only upon the differential Thom class $\hat{U}$ of the orientation. Every differentially oriented $(n+1)$-manifold $M$ determines a section $\int^{\hat{U}}$ of this functor:

$$
\int^{\hat{U}} x \in S^{\hat{U} \mid \partial M}\left(\left.x\right|_{\partial M}\right), \quad \forall x \in \hat{\mathcal{H}}^{n+2}(M) .
$$

\subsection{The Lagrangian field theory}

Let $k=n / 2+1$ for $n$ even. We set

$$
\begin{array}{ccc}
\operatorname{dim} M=n+1, & \operatorname{dim} N=n & \text { dimension } \\
\mathcal{C}_{M, U}=\hat{\mathcal{H}}^{k}(M) \ni x, & \mathcal{C}_{N, V}=\hat{\mathcal{H}}^{k}(N) \ni y & \text { space of fields } \\
S(x)=\int_{M / \mathrm{pt}}^{U} x \cup x, & S(y)=\int_{N / \mathrm{pt}}^{V} y \cup y & \text { action }
\end{array}
$$

Here it should be noted that $2 k=n+2$, so that $x \cup x$ and $y \cup y$ are automatically flat.

Example 11.16. If $n=k=2$, a field $x \in \mathcal{C}_{M, U}$ on a 3-manifold $M$ is a principal $S^{1}$-bundle with connection. If $M$ is closed, $S(x)$ is the $S^{1}$-Chern-Simons invariant of this bundle. On a 2-manifold $N$, a field is again a circle bundle with connection, and the complex lines we obtain may also be constructed from the WZW-functional.

\subsection{Outlook: Differential $K$-theory}

For ordinary cohomology, the differential intersection pairing produces the Chern-Simons invariant, and we have just seen how in a refined viewpoint on differential cohomology the intersection pairing also produces the WZWlines. The goal of this section is to show that the differential intersection pairing for $K$-theory on closed manifolds is non-trivial and also produces a sophisticated concept: it is essentially a certain $\eta$-invariant. One may speculate that the refined differential viewpoint for other generalized cohomology theories leads to other sophisticated invariants which, in the case of manifolds with boundary, take values in objects more fancy than complex lines. 
Rational ordinary differential cohomology may be defined by

$$
\begin{aligned}
& \hat{H}^{k}(M ; \mathbb{Q})=\left\{f \in \operatorname{Hom}_{\mathbf{A b}}\left(Z_{k-1}(M), \mathbb{R} / \mathbb{Q}\right) \mid\right. \\
&\left.\exists \omega \in \Omega^{k}(M) \forall \sigma \in C_{k-1}(M): f(\partial \sigma)=\int_{\sigma} \omega \bmod \mathbb{Q}\right\} .
\end{aligned}
$$

This is a differential cohomology theory in the usual sense for the cohomology theory $E=H \mathbb{Q}$.

Lemma 11.17. Suppose $M=\partial W$ for an oriented compact $n$-dimensional manifold $W$ and let $x \in \hat{H}_{\text {flat }}^{n}(M ; \mathbb{Q})$. Suppose $x$ has an extension to a class $y \in \hat{H}^{n}(W ; \mathbb{Q})$. Viewing $x$ as an element of $H^{n-1}(M ; \mathbb{R} / \mathbb{Q})$, we have

$$
\langle x,[M]\rangle=\int_{W} R(y) \bmod \mathbb{Q}
$$

Proof. By definition, $x([M])=y(\partial[W]) \equiv \int_{W} R(y)$.

Since $H^{n+1}(W, M)=0$ such an extension of $x$ may always be chosen, by the long exact sequence in differential cohomology of the pair. It is unique up to $\hat{H}^{n}(W, M)$.

Suppose given Hermitian vector bundles $E_{i} \rightarrow X$ with connections, $i=$ 1,2 , representing differential $K$-theory classes $x_{i}$. Suppose $X$ is a compact spin manifold of dimension $(4 k+3)$. We write $(x, y)=\int_{X} x \cup y$ for the differential intersection pairing. Then, using the differential Riemann-Roch Theorem, and identifying $\hat{c h}: \hat{K}^{1}(\mathrm{pt}) \stackrel{\cong}{\rightarrow} \hat{H}^{\text {odd }}(\mathrm{pt} ; \mathbb{Q})$,

$$
\left(x_{1}, x_{2}\right) \cong \widehat{\operatorname{ch}}\left(\int_{X} x_{1} \cup x_{2}\right)=\int_{X} \hat{A}(X) \cup \widehat{\operatorname{ch}}\left(x_{1} \cup x_{2}\right) .
$$

The element $\hat{A}(X) \in \hat{H}^{\text {even }}(X ; \mathbb{Q})$ is a refinement of the $\hat{A}$-genus to differential cohomology. The integrand is a sequence of even-degree ordinary differential cohomology classes, but only the component $y$ of degree $\operatorname{dim} X+1$ contributes to the integral. Now if $X$ is a $(4 k+3)$-dimensional compact

\footnotetext{
${ }^{12}$ In [CS85], this group would have been called $\hat{H}^{k-1}(M, \mathbb{R} / \mathbb{Q})$.
} 
spin manifold, then $y$ is automatically flat. If we assume the existence of extensions of $E_{1}, E_{2}$ to $Y, \partial Y=X$, Lemma 11.17 implies

$$
\left(x_{1}, x_{2}\right)=\int_{Y} \hat{A}(Y) \wedge \operatorname{ch}\left(\left(E_{1}, \nabla_{1}\right) \otimes\left(E_{2}, \nabla_{2}\right)\right) \quad \bmod \mathbb{Q} .
$$

By $(4 \cdot 3)$ in APS75, this equals

$$
\operatorname{ind}\left(D_{E_{1} \otimes E_{2}}\right)+\left[h\left(E_{1} \otimes E_{2}\right)+\eta\left(E_{1} \otimes E_{2}\right)\right] / 2,
$$

where the index is taken of the twisted Dirac operator (with respect to certain boundary conditions), $h$ denotes the dimension of the space of harmonic spinors for the restriction of $D_{E_{1} \otimes E_{2}}$ to $X$ and $\eta=\eta(0)$ its eta-invariant. Of course, the index is an integer and vanishes in $\mathbb{R} / \mathbb{Q}$, as does $h / 2$.

In summary, the intersection pairing $(\bmod \mathbb{Q})$ is essentially the etainvariant of the corresponding twisted Dirac operator on $X$. In particular, this expression $(\bmod \mathbb{Q})$ depends only on $X$ (being the differential intersection pairing on $X$ ), a fact that can also be deduced directly from the Index Theorem.

Corollary 11.18. The differential intersection pairing in differential $K$ theory is non-trivial in certain cases.

\section{A APPEndix}

\section{A.1 Braided Monoidal Categories}

\section{A.1.1 Adjunctions}

Definition A.1. An adjunction $(F, G, \varepsilon, \eta)$ consists of

1. Functors $F: \mathcal{C} \rightarrow \mathcal{D}$ and $G: \mathcal{D} \rightarrow \mathcal{C}$,

2. Natural transformations $\varepsilon: F G \rightarrow \mathrm{id}_{\mathcal{D}}, \eta: \mathrm{id}_{\mathcal{C}} \rightarrow G F$.

These are required to satisfy the zig-zag identities:

$$
\operatorname{id}_{F}: F \stackrel{F \eta}{\longrightarrow} F G F \stackrel{\varepsilon F}{\longrightarrow} F, \quad \operatorname{id}_{G}: G \stackrel{\eta G}{\longrightarrow} G F G \stackrel{G \varepsilon}{\longrightarrow} G .
$$


The definition of adjunction makes sense in any bicategory, for example in MonCat. An adjunction is said to be an adjoint equivalence in case $\varepsilon, \eta$ are natural isomorphisms. An equivalence in a bicategory consists of the data $(F, G, \varepsilon, \eta)$ as above with $\varepsilon, \eta$ natural isomorphisms, but we do not require the zig-zag identities. Then $\left(G, F, \eta^{-1}, \varepsilon^{-1}\right)$ is another equivalence and similarly for adjoint equivalences.

An important feature of Cat is that a functor $F$ may be placed inside an (adjoint) equivalence precisely when it is essentially surjective and fully faithful.

\section{A.1.2 Monoidal Categories, Functors, and Transformations}

[JS93, ML98], and [Lei04] are good references for (braided) monoidal categories.

Definition A.2. A monoidal category is an ordinary category $\mathcal{C}$ along with a functor

$$
\otimes: \mathcal{C} \times \mathcal{C} \rightarrow \mathcal{C}
$$

an object $1 \in \mathcal{C}$, and natural isomorphisms

$$
\begin{array}{rrr}
a_{X, Y, Z}:(X \otimes Y) \otimes Z & \rightarrow X \otimes(Y \otimes Z) & \text { (associator) } \\
\lambda_{X}: 1 \otimes X & \rightarrow X \\
\rho_{X}: X \otimes 1 & \rightarrow X & \text { (unit constraints) }
\end{array}
$$

satisfying the following axioms:

Mac Lane's Pentagon: 


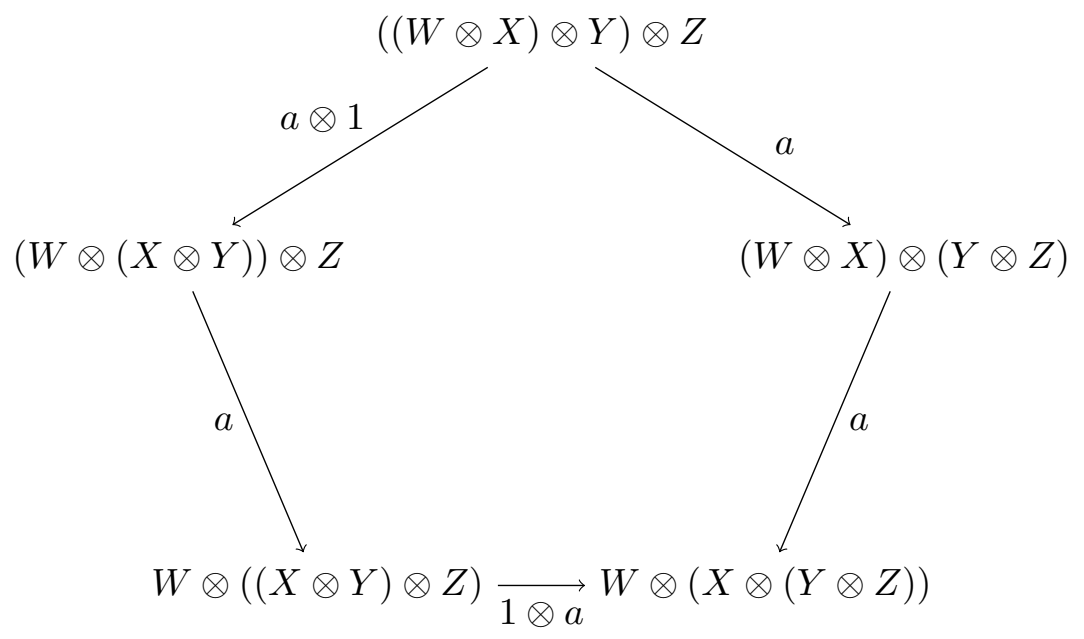

Triangle Identities:
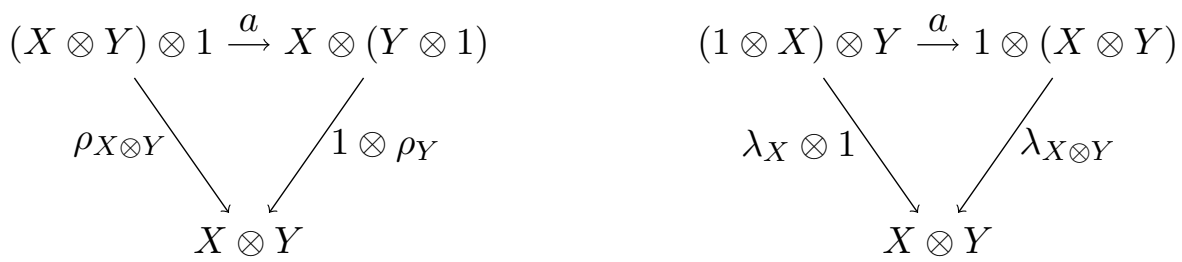

If the associativity and unit constraints are all the identity, one speaks of a strict monoidal category.

Example A.3. From a cochain complex $(C, \delta)$ one may construct a strict symmetric monoidal category $\mathscr{C}^{n}$ in degree $n$ as follows. As objects we take $\mathrm{Ob}(\mathscr{C})=C^{n}$. A morphism from $x \in C^{n}$ to $y \in C^{n}$ consists of a $\operatorname{im}(\delta)$-coset of elements $u \in C^{n-1}$ with $\delta u=x-y$, and composition is given by addition. The monoidal structure is determined by addition as well, and the symmetry is taken as the identity.

The full subcategory $\mathscr{Z}^{n} \hookrightarrow \mathscr{C}$ on objects $Z^{n}=\left\{x \in C^{n} \mid \delta x=0\right\}$ will also occasionally be used.

A functor between monoidal categories carries extra data: 
Definition A.4. Suppose $\mathcal{C}, \mathcal{D}$ denote monoidal categories. A functor

$$
F: \mathcal{C} \rightarrow \mathcal{D}
$$

along with an isomorphism $F_{1}: 1 \rightarrow F(1)$ and natural isomorphisms

$$
F_{X, Y}: F(X) \otimes F(Y) \rightarrow F(X \otimes Y)
$$

is called a monoidal functor in case the following diagrams commute:
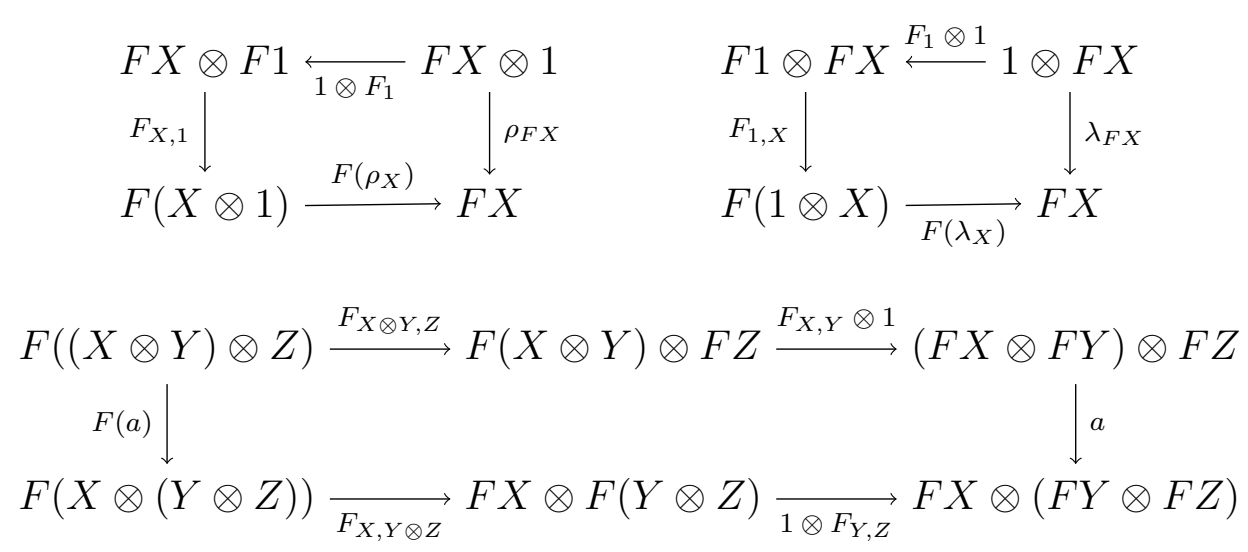

In case all $F_{X, Y}, F_{1}$ are the identity, we speak of a strict monoidal functor. Monoidal functors $F: \mathcal{C} \rightarrow \mathcal{D}$ and $G: \mathcal{D} \rightarrow \mathcal{E}$ are composed as follows:

$$
\begin{aligned}
& (G F)_{X, Y}: G F(X) \otimes G F(Y) \stackrel{G_{F X, F Y}}{\longrightarrow} G(F X \otimes F Y) \stackrel{F_{X, Y}}{\longrightarrow} G F(X \otimes Y), \\
& (G F)_{1}: 1 \stackrel{G_{1}}{\longrightarrow} G(1) \stackrel{G\left(F_{1}\right)}{\longrightarrow} G(F(1)) .
\end{aligned}
$$

Along with the identity functor id (strict) we obtain the category MonCat. Like Cat, this is in fact a bicategory:

Definition A.5. Suppose $F, G: \mathcal{C} \rightarrow \mathcal{D}$ are monoidal functors. A monoidal transformation $u: F \rightarrow G$ is a natural transformation $u_{X}: F X \rightarrow G X$ (of ordinary functors) such that the following diagrams commute:
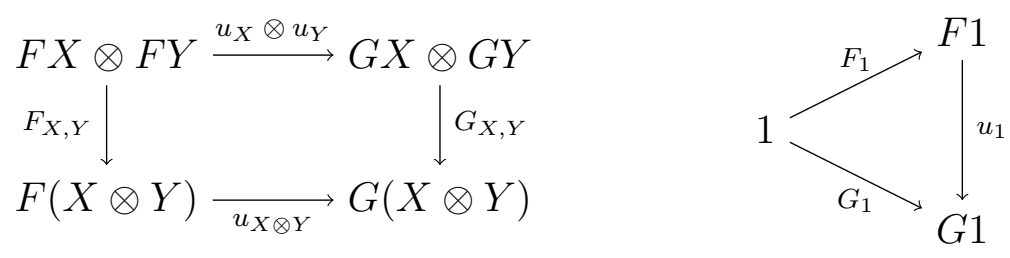
Note that, unlike a monoidal functor, a monoidal transformation has no additional data. Since we work only with strong monoidal functors we have the following:

Theorem A.6 (Doctrinal Adjunction [Kel74). Suppose $(F, G, \varepsilon, \eta)$ is an adjoint equivalence and that $F: \mathcal{C} \rightarrow \mathcal{D}$ is a monoidal functor. Then there exists a unique monoidal structure on $G$ turning $(F, G, \varepsilon, \eta)$ into an adjoint equivalence in MonCat.

Proof. That $\varepsilon, \eta$ are monoidal transformations means

$$
\begin{aligned}
\eta_{X \otimes Y} & =G\left(F_{X, Y}\right) \circ G_{F X, F Y} \circ\left(\eta_{X} \otimes \eta_{Y}\right), \\
\varepsilon_{A} \otimes \varepsilon_{B} & =\varepsilon_{A, B} \circ F\left(G_{A, B}\right) \circ F_{G A, G B}, \\
\eta_{1} & =G\left(F_{1}\right) \circ G_{1}, \\
\mathrm{id} & =\varepsilon_{1} \circ F\left(G_{1}\right) \circ F_{1} .
\end{aligned}
$$

Since $F$ is faithful this clearly determines $G_{A, B}, G_{1}$ uniquely. Conversely, the second and forth equations may be used to define these isomorphisms. The other two equations are then consequences of the zig-zag identities and naturality. Compatibility with the associativity and unit constraints derive from the corresponding properties of $F$, the zig-zag identities, and naturality. For more detail, see [Kel74].

\section{A.1.3 Braids}

Definition A.7. Suppose $\mathcal{C}$ is a monoidal category. A braid on $\mathcal{C}$ is a natural family of isomorphisms

$$
s_{X, Y}: X \otimes Y \rightarrow Y \otimes X
$$

making the following two hexagons commute:

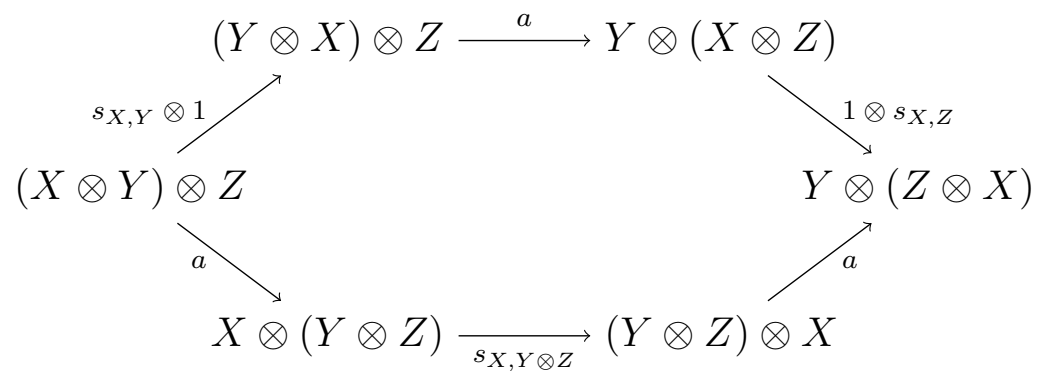




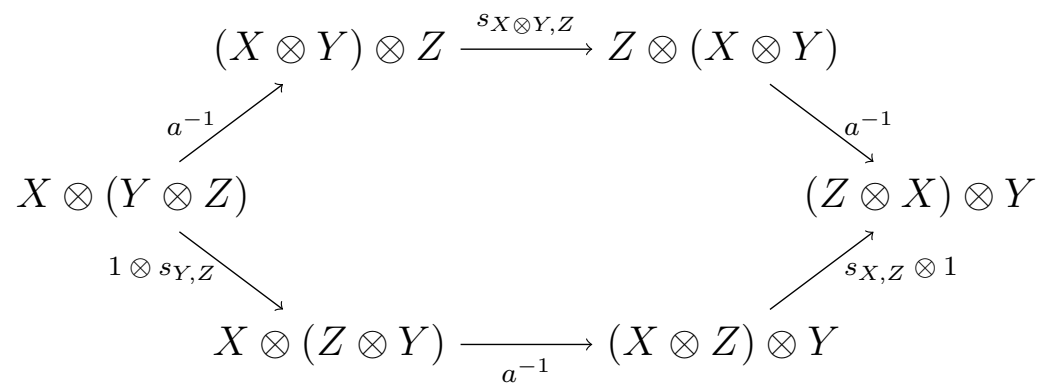

A monoidal category together with a braid is called a braided monoidal category. The hexagon implies that we have the following compatibility with the unit [JS93, Proposition 2.1]:

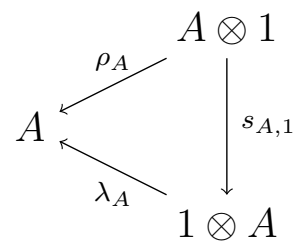

Definition A.8. A monoidal functor $F: \mathcal{C} \rightarrow \mathcal{D}$ between braided monoidal categories is said to be braided in case the following diagram commutes:

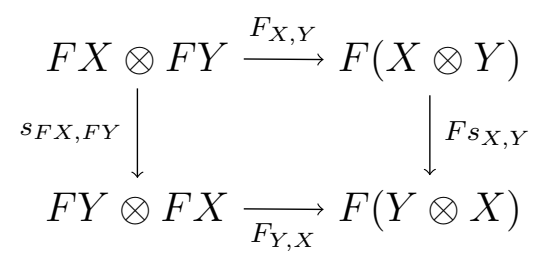

A monoidal transformation between braided monoidal functors is called a braided monoidal transformation (no further conditions imposed).

\section{A.2 Monoidal Structures under Equivalences}

In this section we collect some of the more sophisticated facts on monoidal categories for later reference. They all reflect that the notion of monoidal category is the 'correct' weakening of the notion of a strict monoid in Cat and concern the behavior of these notions under various types of equivalences. 


\section{A.2.1 Structure on Categories under Equivalences}

Proposition A.9. Suppose that $F: \mathcal{C} \rightarrow \mathcal{D}$ is an equivalence of categories and that $\mathcal{C}$ or $\mathcal{D}$ is monoidal. Then there exists a monoidal structure on the other category, turning $F$ into a monoidal equivalence.

Of course, the monoidal structure is then unique up to equivalence. There is, however, no canonical choice in this equivalence class.

Proof. Placing $F$ in an adjoint equivalence $(F, G, \varepsilon, \eta)$, doctrinal adjunction implies that we may assume $\mathcal{D}$ to be monoidal. It is possible to verify directly that $C_{1} \otimes C_{2}=G\left(F C_{1} \otimes F C_{2}\right)$ defines a monoidal structure on $\mathcal{C}$. The associativity and unit constraints are constructed by applying $G$ to those of $\mathcal{D}$ and using $\varepsilon, \eta$. Let me outline a different proof: let's call a pair $(X, \varphi)$ of

$$
X \in \mathcal{C}, \quad \varphi: F C_{1} \otimes F C_{2} \stackrel{\cong}{\longrightarrow} F X
$$

'a' tensor product of $C_{1}, C_{2} \in \mathcal{C}$. For any two choices $\left(X_{1}, \varphi_{1}\right),\left(X_{2}, \varphi_{2}\right)$ of tensor product of $C_{1}, C_{2}$ the full faithfulness of $F$ implies that there is a unique isomorphism

$$
f: X_{1} \stackrel{\cong}{\longrightarrow} X_{2} \text { with } \quad \varphi_{2}=F(f) \circ \varphi_{1} .
$$

The functor $F$ is essentially surjective, so we may choose tensor products $C_{1} \odot C_{2}$ for every pair of objects:

$$
F_{C_{1}, C_{2}}: F C_{1} \otimes F C_{2} \stackrel{\cong}{\longrightarrow} F\left(C_{1} \odot C_{2}\right)
$$

Since $F$ is fully faithful, $\odot$ may be viewed uniquely as a functor $\mathcal{C} \times \mathcal{C} \rightarrow \mathcal{C}$. In detail, $f_{1} \odot f_{2}$ is the unique dotted arrow making the diagram

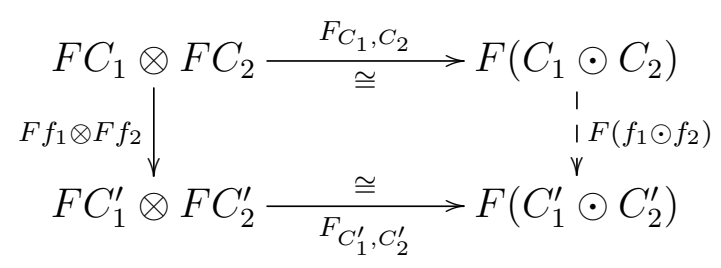

commute. Pick also an element $1_{\mathfrak{C}} \in \mathcal{C}$ with an isomorphism

$$
F_{1}: 1_{\mathcal{D}} \cong F\left(1_{\mathcal{C}}\right) .
$$


The constraints $\lambda, \rho, \alpha$ in $\mathcal{C}$ are obtained uniquely from those in $\mathcal{D}$ by requiring that the diagrams of Definition A.4 commute. Their naturality follows from the faithfulness of $F$ and the corresponding naturality in $\mathcal{D}$.

It remains to prove the commutativity of Mac Lane's Pentagon and the triangle equalities in $\mathcal{C}$. Using the faithfulness of $F$ this may be checked after applying $F$, which results in diagrams which may be identified with the corresponding diagrams in $\mathcal{D}$.

The previous proposition refines to braided monoidal categories:

Proposition A.10. Let $F: \mathcal{C} \rightarrow \mathcal{D}$ be a monoidal equivalence. If $\mathcal{D}$ is braided monoidal, one may define a canonical braided monoidal structure on $\mathcal{C}$ that turns $F$ into a braided monoidal equivalence. If $\mathcal{C}$ is braided monoidal, $\mathcal{D}$ has a braided monoidal structure making $F$ braided, but it is not unique.

Proof. The braid

$$
F\left(C_{1} \odot C_{2}\right) \cong F\left(C_{1}\right) \otimes F\left(C_{2}\right) \rightarrow F\left(C_{2}\right) \otimes F\left(C_{1}\right) \cong F\left(C_{2} \odot C_{1}\right)
$$

is covered by a unique isomorphism $C_{1} \odot C_{2} \rightarrow C_{2} \odot C_{1}$. The three coherence conditions are verified by applying $F$ and identifying the resulting diagram with the corresponding diagram in $\mathcal{D}$.

For the second part note that one may choose an inverse monoidal equivalence of $F$ [Lei04, Proposition 1.2.14]. Now apply the first part.

\section{A.2.2 Structure on Functors under Equivalences}

Recall that for a monoidal functor to be braided is a property, namely compatibility with the braid, not additional data.

Proposition A.11. Let $\phi_{1}: \mathcal{C}_{1} \stackrel{\sim}{\rightarrow} \mathcal{D}_{1}, \phi_{2}: \mathcal{C}_{2} \stackrel{\sim}{\rightarrow} \mathcal{D}_{2}$ be braided monoidal equivalences. Suppose we have a diagram of monoidal functors

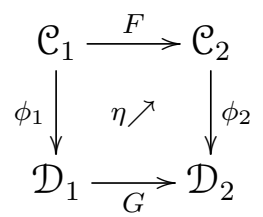


which commutes up to a monoidal natural isomorphism $\eta: G \phi_{1} \rightarrow \phi_{2} F$. Suppose that $G$ is a braided monoidal functor. Then $F$ is braided monoidal as well.

Proof. We must check the commutativity of the diagram in $\mathrm{C}_{2}$

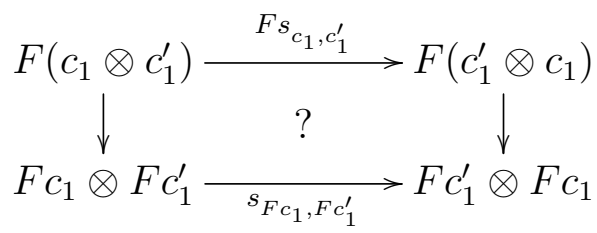

for which we apply $\phi_{2}$ to it. The resulting diagram may be identified (using the definition of braid on $\mathcal{C}_{2}$, the given natural isomorphism, and the compatibility of $G$ with the symmetry) with a diagram which is the image under $G$ of the compatibility of $\phi_{1}$ with $s$ :

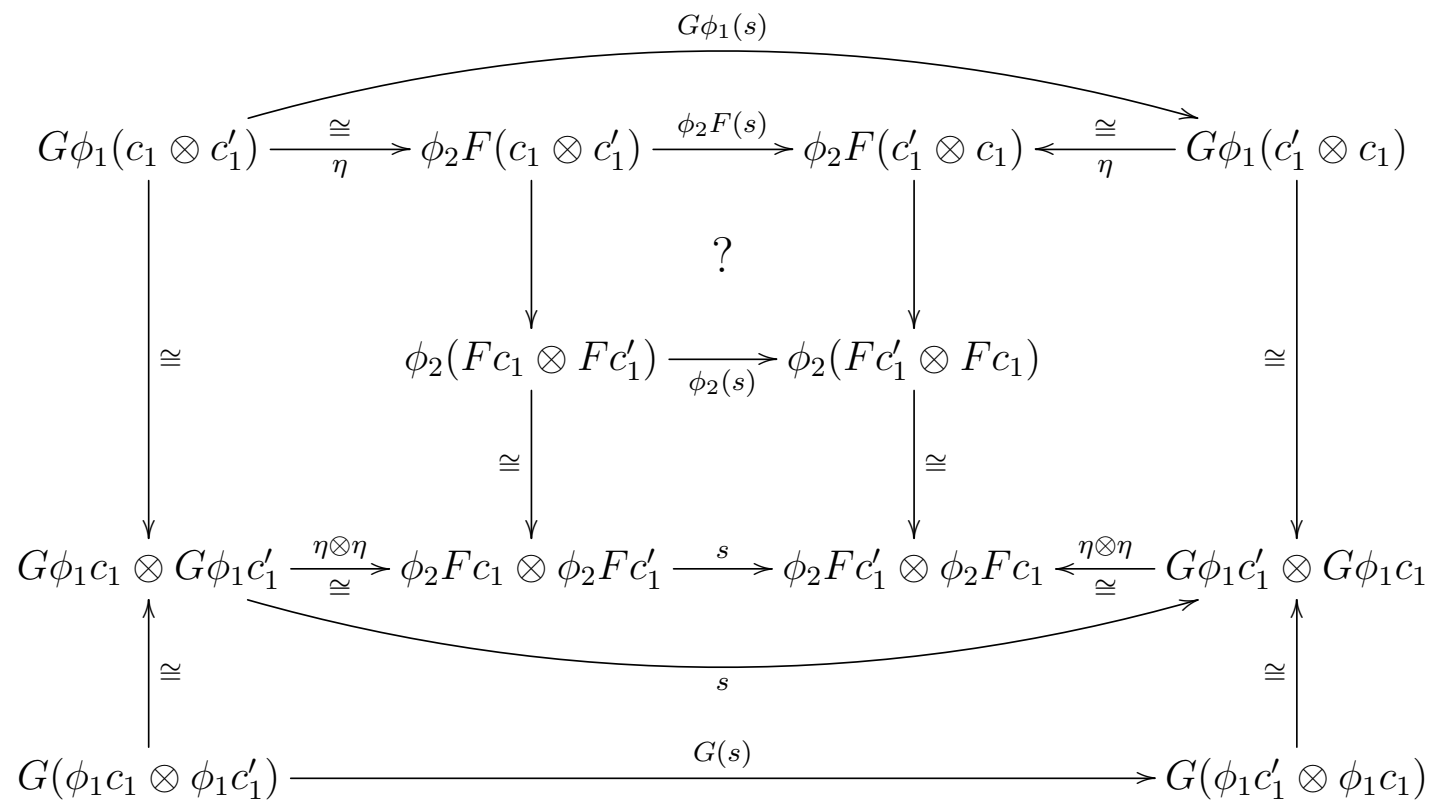

All diagrams, including the outer square, commute. It follows that the diagram marked '?' commutes as well.

A version in which $F$ is assumed to be braided monoidal is immediately deduced by applying Proposition A.11 to inverse equivalences of $\phi_{1}, \phi_{2}$. 
Lemma A.12. Suppose $F: \mathcal{C} \rightarrow \mathcal{D}$ is a monoidal functor and let $u: F \rightarrow$ $G$ be a natural isomorphism to an ordinary functor $G$. There is a unique monoidal structure on $G$ making u a monoidal transformation.

Proposition A.13. Given a commutative diagram in Cat, $G \circ F=H$,

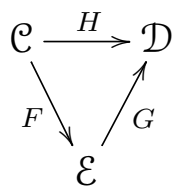

in which $F, H$ are monoidal functors and $F$ is an equivalence. Then there exists a unique structure of monoidal functor on $G$, making the previous diagram commute in MonCat.

Proof. If a monoidal structure on $G$ exists, we must have

$$
H_{C_{1}, C_{2}}=G\left(F_{C_{1}, C_{2}}\right) \circ G_{F C_{1}, F C_{2}}, \quad H_{1}=G\left(F_{1}\right) \circ G_{1},
$$

so $G_{E_{1}, E_{2}}$ is determined on the image of $F$. For general $E_{1}, E_{2} \in \mathcal{E}$ pick isomorphisms $\varphi_{i}: E_{i} \rightarrow F C_{i}$. By naturality we have $G_{F C_{1}, F C_{2}} \circ\left(G \varphi_{1} \otimes G \varphi_{2}\right)=$ $G\left(\varphi_{1} \otimes \varphi_{2}\right) \circ G_{E_{1}, E_{2}}$. We conclude that the monoidal structure on $G$ is unique. To prove that such a structure exists, place $F$ in an adjoint equivalence $(F, R, \eta, \varepsilon)$ which, by doctrinal adjunction, may uniquely be viewed as an adjoint equivalence in MonCat. We have a natural isomorphism

$$
G(\varepsilon): H R=G F R \stackrel{\cong}{\longrightarrow} G .
$$

According to Lemma A.12, there is a unique monoidal structure on $G$ making $G(\varepsilon)$ a monoidal transformation. The composition of a monoidal transformation with a monoidal functor is again monoidal. Therefore

$$
H(\eta): H \rightarrow H R F, \quad G(\varepsilon) F: H R F \rightarrow G F
$$

are monoidal transformations which compose to the identity, by the zig-zag identities. But this just means $H=G F$ in MonCat.

By a dual argument we have: 
Proposition A.14. Given a commutative diagram in Cat, $G \circ F=H$,

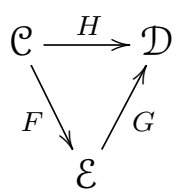

in which $G, H$ are monoidal functors and $G$ is an equivalence. Then there exists a unique structure of monoidal functor on $F$, making the previous diagram commute in MonCat.

\section{A.3 Simplicial Sets}

Before we begin, let us quickly establish essentially the notation of GJ09 from simplicial homotopy theory. Let $\Delta \subset$ Cat denote the full skeletal subcategory on all finite ordinals $[n]=0 \rightarrow \cdots \rightarrow n, n \geq 0$. Recall that a simplicial set is a functor

$$
X: \Delta^{\mathrm{op}} \rightarrow \text { Set. }
$$

Define the category of $\operatorname{simplicial}$ sets as $\operatorname{Set}_{\Delta}=\operatorname{Fun}\left(\Delta^{\mathrm{op}}\right.$, Set $)$. Small limits and colimits are formed level-wise and all exist. Since every morphisms in $\Delta$ may by written as compositions of codegeneracy maps

$$
s^{i}:[n-1] \rightarrow[n] \quad(\text { skip } i \text { in the image) }
$$

and coface maps

$$
d^{i}:[n] \rightarrow[n-1] \quad \text { (repeat } i \text { twice) }
$$

this amounts to the classical definition of a simplicial set (as a family of sets $X_{n} \in$ Set along with maps $s_{i}, d_{i}$ between them satisfying various relations).

The most common simplicial sets are the standard simplices which may be organized into a functor

$$
\Delta \rightarrow \text { Set }_{\Delta}, \quad[n] \mapsto \Delta^{n}=\Delta(-,[n]) .
$$

By the Yoneda Lemma, $\operatorname{Set}_{\Delta}\left(\Delta^{n}, X\right) \cong X_{n}$. Another important class of simplicial sets are the horns,

$$
\Lambda_{k}^{n} \subset \Delta^{n},
$$


which is the smallest simplicial subset of $\Delta^{n}$ containing the image of $d^{i}$ : $\Delta^{n-1} \rightarrow \Delta^{n}$ for every $i \neq k$.

A fibration is a simplicial map $p: X \rightarrow Y$ for which we may always provide the dotted arrow in all diagrams of the form $(n \in \mathbb{N}, 0 \leq k \leq n)$

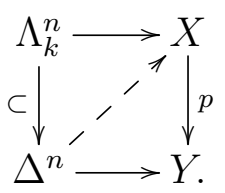

A level-wise monomorphism $A \rightarrow B$ is called a cofibration. Any simplicial set $X$ with base-point $x \in X_{0}$ has (functorial) homotopy groups $\pi_{*}(X, x)$ (a set for $*=0$ ). These may be defined as the usual homotopy groups of a geometric realization of $X$, which is the following coend (more commonly known as a certain coequalizer) in CGHaus (the category of compactly generated Hausdorff spaces):

$$
|X|=\int^{[n] \in \Delta} X_{n} \times\left|\Delta^{n}\right|,
$$

where $\left|\Delta^{n}\right|=\left\{\left(t_{0}, \ldots, t_{n}\right) \in \mathbb{R}^{n+1} \mid \sum_{i=0}^{n} t_{i}=1, t_{i} \geq 0\right\}$.

A simplicial map $f: X \rightarrow Y$ is called a weak equivalence if $|f|:|X| \rightarrow|Y|$ is a weak equivalence of topological spaces:

1. $\pi_{0}|f|: \pi_{0}|X| \rightarrow \pi_{0}|Y|$ is a bijection.

2. For every $x \in|X|$ and $n \geq 1, \pi_{n}|f|$ is an isomorphism.

For Kan complexes $X, Y$, this may be checked also by using simplicial homotopy groups.

Theorem A.15 ([GJ09, Theorem I.11.3]). The category Set $_{\Delta}$ with the above cofibration, fibrations, and weak equivalence forms a model category.

The geometric realization $|\cdot|:$ Set $_{\Delta} \rightarrow$ CGHaus has a right adjoint $S=\operatorname{sing}$, the singular set of a topological space. Its $n$-simplices are given by

$$
(\operatorname{sing} X)_{n}=\left\{\sigma:\left|\Delta^{n}\right| \rightarrow X \text { continuous }\right\}
$$


Since the pair $(|\cdot|, S)$ is a Quillen equivalence ([GJ09, Theorem I.11.4]), to practice homotopy theory in any of these two categories is generally regarded as being equivalent.

Fibrant objects in this model category are called Kan complexes. Note that every object in $\mathbf{S e t}_{\Delta}$ is cofibrant. The category $\mathbf{S e t}_{\Delta}$ has an internal Hom given by

$$
\operatorname{Hom}_{\mathbf{S e t}_{\Delta}}(X, Y)_{n}=\operatorname{Set}_{\Delta}\left(X \times \Delta^{n}, Y\right) .
$$

We may therefore view $\mathbf{S e t}_{\Delta}$ as a category enriched in $\mathbf{S e t}_{\Delta}$, and it is in fact even a simplicial model category (so Quillen's axiom SM7 is satisfied Qui67).

The standard subdivision of the prism [GJ09, p.17] will appear a lot. It is given by simplicial maps

$$
h_{j}: \Delta^{n+1} \rightarrow \Delta^{n} \times \Delta^{1}, \quad j=0, \ldots, n,
$$

which are determined by the map $[n+1] \rightarrow[n] \times[1]$ as in the diagram

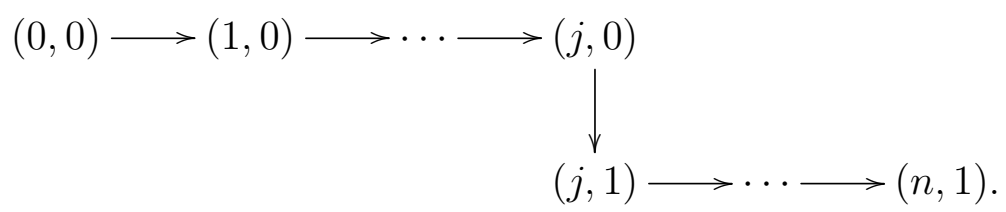

This diagram determines the boundary of the embedded $(n+1)$-simplex $h_{j}$ in $\Delta^{n} \times \Delta^{1}$.

The homotopy groups of a pointed simplicial set $K$ are defined in terms of their geometric realizations:

$$
\pi_{k}(K, k)=\pi_{k}(|K|, k) .
$$

It is an important fact that for a pointed Kan complex $K$, we have the following combinatorial description of the homotopy groups (sometimes called simplicial homotopy groups):

$$
\pi_{k}(K, \mathrm{pt})=\left\{f \in \operatorname{Set}_{\Delta}\left(\Delta^{k}, K\right) \mid f\left(\partial \Delta^{k}\right)=\{\mathrm{pt}\}\right\} / \simeq
$$

Here, $f \simeq g$ if there exists $h: \Delta^{k} \times \Delta^{1} \rightarrow K$ with

1. $h\left|\Delta^{k} \times\{0\}=f, h\right| \Delta^{k} \times\{1\}=g$,

2. $h\left(\partial \Delta^{k} \times \Delta^{1}\right)=\{\mathrm{pt}\}$. 


\section{A.3.1 Simplicial Loop Space}

The purpose of this subsection is to exhibit an isomorphism between two definitions for the simplicial loop space (113). While the standard construction $\operatorname{Hom}_{\text {Set }_{\Delta}^{+}}\left(S^{1}, K\right)$ has nice adjointness properties, the other definition is more explicit and allows to view loops as certain coequalizer diagrams, which is sometimes useful.

In the following we will identify maps $\theta:[n] \rightarrow[m]$ with their pushforwards $\theta_{*}: \Delta^{n} \rightarrow \Delta^{m}$.

Lemma A.16. Every $\theta=\left(\theta_{1}, \theta_{2}\right): \Delta^{n+1} \rightarrow \Delta^{k} \times \Delta^{1}$ for which there exists $i$ with $\theta_{2}(i)=0, \theta_{2}(i+1)=1$ factors uniquely as

$$
\theta: \Delta^{n+1} \stackrel{\hat{\theta}}{\rightarrow} \Delta^{k+1} \stackrel{h_{j}}{\rightarrow} \Delta^{k} \times \Delta^{1} .
$$

In this case, $j=\theta_{1}(i)$.

Proof. We first prove uniqueness. By assumption, $\operatorname{pr}_{2} \theta$ is the push-forward of $[n+1] \rightarrow[1], i \mapsto 0, i+1 \mapsto 1$. By (111),

$$
\operatorname{pr}_{2} h_{j} \hat{\theta}(i)=0, \quad \operatorname{pr}_{2} h_{j} \hat{\theta}(i+1)=1,
$$

which implies $\hat{\theta}(i)=j$ and $j=s^{j}(j)=s^{j} \hat{\theta}(i)=\operatorname{pr}_{1} \theta(i)=\theta_{1}(i)$. Applying $\mathrm{pr}_{1}$ to 111 yields

$$
s^{j} \hat{\theta}=\operatorname{pr}_{1} h_{j} \hat{\theta}=\theta_{1} .
$$

It follows that for all $r: \theta_{1}(r)<j$ we have $\hat{\theta}(r)=\theta_{1}(r)$, for all $r: \theta_{1}(r)>j$ we have $\hat{\theta}(r)-1=\theta_{1}(r)$, and that for all $r \in \theta_{1}^{-1}(j)$ we have $\hat{\theta}(r) \in\{j, j+1\}$. By $(112), \hat{\theta}(i)=j, \hat{\theta}(i+1) \neq j$. In case $\theta_{1}(i+1)=j$ we have $\hat{\theta}(i+1)=j+1$ which of course determines $\hat{\theta}$ on $\theta_{1}^{-1}(j)$ as well. In case $\theta_{1}(i+1)>j, i$ is the largest element of $\theta_{1}^{-1}(j)$ so that $\hat{\theta}$ takes $\theta_{1}^{-1}(j)$ to elements $\leq \hat{\theta}(i)=j$, i.e. is constant. This implies the uniqueness of $\hat{\theta}$. Conversely, this prescription proves the existence of $\hat{\theta}$ as well.

Lemma A.17. For every $\tau: \Delta^{n+1} \rightarrow \Delta^{k+1}$ and $i$ with $\tau(i+1)=\tau(i)+1$ 
there exists a unique factorization ( $a$ unique $j$ and $\sigma$ )

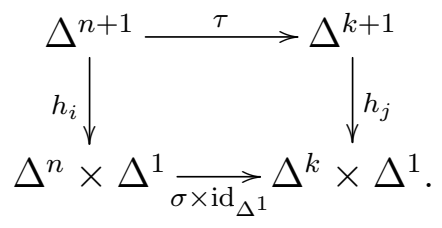

Moreover, $j=\tau(i)$.

Proof. To prove uniqueness, observe that $\operatorname{pr}_{2} h_{j} \tau=\operatorname{pr}_{2}\left(\sigma \times \operatorname{id}_{\Delta^{1}}\right) h_{i}$ implies that $\tau(i)=j, \tau(i+1) \neq j$. Composing with $\operatorname{pr}_{1}$ leads to $s^{j} \tau=\sigma s^{i}$. Thus $\sigma=\sigma s^{i} d^{i}=s^{j} \tau d^{i}$ is unique, which also proves existence.

Corollary A.18. Let $(K, *) \in$ Set $_{\Delta}^{*}$ be a pointed simplicial set. A sequence of $(n+1)$-simplices $f_{0}, \ldots, f_{n} \in K_{n+1}$ satisfying

$$
d_{0} f_{0}=*=d_{n+1} f_{n}, \quad d_{j} f_{j}=d_{j} f_{j-1}(1 \leq j \leq n)
$$

uniquely determines an n-simplex $f \in \Omega(K, *)_{n}=\mathbf{H o m}_{\text {Set }_{\Delta}^{+}}\left(S^{1}, K\right)_{n} \subset$ $\operatorname{Set}_{\Delta}\left(\Delta^{n} \times \Delta^{1}, K\right)$. Moreover, $f_{j}=f h_{j}$. If we define a simplicial set $\Omega^{\operatorname{simp}} K$ having as $n$-simplices all sequences $f_{0}, \ldots, f_{n} \in K_{n+1}$ as above and with face and degeneracy maps

$$
\begin{aligned}
& d_{i}\left(f_{0}, \ldots, f_{n}\right)=\left(d_{i+1} f_{0}, \ldots, d_{i+1} f_{i-1}, d_{i} f_{i+1}, \ldots, d_{i} f_{n}\right), \\
& s_{i}\left(f_{0}, \ldots, f_{n}\right)=\left(s_{i+1} f_{0}, \ldots, s_{i+1} f_{i}, s_{i} f_{i}, \ldots, s_{i} f_{n}\right),
\end{aligned}
$$

then we have an isomorphism $\phi: \Omega(K) \cong \Omega^{\text {simp }} K$ of simplicial sets.

Proof. We must associate a value $f(\sigma)$ to every simplex $\sigma: \Delta^{k} \rightarrow \Delta^{n} \times \Delta^{1}$. If $\operatorname{pr}_{2} \sigma$ is constant, we set $f(\sigma)=*$. Else we may factor

$$
\sigma: \Delta^{k} \stackrel{\hat{\sigma}}{\rightarrow} \Delta^{n+1} \stackrel{h_{j}}{\rightarrow} \Delta^{n} \times \Delta^{1} .
$$

Now set $f(\sigma)=\hat{\sigma}^{*} f_{j}$. The inverse $\phi$ of this association is given by $\phi(f)=$ $\left(f h_{0}, \ldots, f h_{n}\right)$. The compatibility of $\phi$ with the face an degeneracy maps now follows from the compatibility of the subdivision of the prism maps $h_{i}$ 
with faces and degeneracies:

$$
\begin{aligned}
h_{j} s^{i} & =\left(s^{i} \times 1\right) h_{j+1} & & (i \leq j) \\
\left(s^{j} \times 1\right) h_{i} & =h_{i} s^{j+1} & & (i \leq j) \\
\left(d^{i} \times 1\right) h_{j-1} & =h_{j} d^{i} & & (i<j) \\
h_{j} d^{j+1} & =h_{j+1} d^{j+1} & & (i \geq j+1) . \\
\left(d^{i} \times 1\right) h_{j} & =h_{j} d^{i+1} & &
\end{aligned}
$$

\section{A.4 $\infty$-Categories}

For the convenience of the reader, we shall give in this section a rapid account of those parts of the theory of $\infty$-categories which are relevant to our discussion. The definite reference for these matters is [Lur09].

Definition A.19. An inner fibration $p: K \rightarrow L$ between simplicial sets is a map which has the right lifting property (RLP) for all inner horn inclusions $\Lambda_{i}^{n} \subset \Delta^{n}, 0<i<n$.

An $\infty$-category $\mathcal{C}$ is a simplicial set for which the map $\mathcal{C} \rightarrow \Delta^{0}$ is an inner fibration. That is, every inner horn $\Lambda_{i}^{n} \rightarrow \mathcal{C}$ admits some filler $\Delta^{n} \rightarrow \mathcal{C}$. An $\infty$-category is a generalization of an ordinary category in the sense that the nerve $N$ : Cat $\rightarrow$ Set $_{\Delta}$, which is a fully faithful embedding, has image those simplicial sets that admit unique inner horn fillers.

An edge $\phi \in \mathcal{C}_{1}$ is called a morphism in $\mathcal{C}$ and said to have domain $d_{1} \phi$ and codomain $d_{0} \phi$. For example, $\mathrm{id}_{c}=s_{0} c$ is called the identity of $c \in \mathfrak{C}_{0}$.

Of course, these kinds of morphisms may not be composed. We will, however, for any $\sigma: \Delta^{2} \rightarrow \mathcal{C}$ regard $d_{1} \sigma$ as a composition of $d_{0} \sigma$ and $d_{2} \sigma$ and present $\sigma$ as a witness for it.

\section{A.4.1 The Simplicial Nerve}

The above approach to higher categories is essentially equivalent to that of simplicial categories $\mathrm{Cat}_{\Delta}$ and stands in close relationship to the latter. There is a functor, due to Cordier,

$$
\mathfrak{C}: \operatorname{Set}_{\Delta} \rightarrow \mathrm{Cat}_{\Delta} .
$$


As observed in [DS11, the simplicial category $\mathfrak{C}[S]$ may be described naturally as a generalization of the free simplicial resolution of an ordinary category: $\operatorname{Ob}(\mathfrak{C}[S])=S_{0}$ and for $X, Y \in S_{0}$ the $n$-simplices $\mathfrak{C}[S](X, Y)_{n}$ are the $(n+1)$-fold bracketings of necklaces in $S$ from $X$ to $Y$. To explain, a necklace from $X$ to $Y$ in $S$ is a simplicial map from a pushout, for some $k \geq 0$ and each $n_{i}>0$,

$$
f: \Delta^{n_{0}} \vee \Delta^{n_{1}} \vee \cdots \vee \Delta^{n_{k}} \rightarrow S
$$

where $\Delta^{n_{i}}$ is glued to $\Delta^{n_{i+1}}$ by attaching the last vertex $n_{i} \in \Delta^{n_{i}}$ to the first vertex $0 \in \Delta^{n_{i+1}}$ so that each $f \mid \Delta^{n_{i}}$ is a non-degenerate simplex. The joints $J$ of the necklace are the attaching vertices. The initial joint maps to $X$ while the final joint maps to $Y$. An $(n+1)$-fold bracketing is a flag of subsets of the vertices

$$
J \subset T^{0} \subset \cdots \subset T^{n} \subset\left(\Delta^{n_{0}} \vee \Delta^{n_{1}} \vee \cdots \vee \Delta^{n_{k}}\right)_{0}
$$

The face and degeneracy maps are simply given by leaving out or doubling terms in this flag. Composition is by concatenation of necklaces. More formally, one may also define $\mathfrak{C}$ first only on simplices $\Delta^{n}$ and then extend. This is the approach in Lur09]

The functor $\mathfrak{C}$ has three important applications: first, we obtain a notion of mapping space

$$
\operatorname{Map}_{\mathcal{C}}(X, Y)=\mathfrak{C}[\mathcal{C}](X, Y) \in \operatorname{Set}_{\Delta}
$$

for $\infty$-categories $\mathcal{C}$. Secondly, by retaining only the path components, we may define the homotopy category he of an $\infty$-category $\mathcal{C}$ :

$$
\mathrm{h} \mathcal{C}= \begin{cases}\text { Objects: } & \mathrm{C}_{0}, \\ \text { Morphisms: } & \mathrm{h} \mathcal{C}(X, Y):=\pi_{0} \operatorname{Map}_{\mathcal{C}}(X, Y) .\end{cases}
$$

An isomorphic category may be constructed by the usual fundamental groupoid construction [Lur09, 1.2.3.9]. Finally, the functor $\mathfrak{C}$ admits a right adjoint, the simplicial nerve

$$
N: \mathbf{C a t}_{\Delta} \rightarrow \operatorname{Set}_{\Delta},
$$

which is defined as $N(\mathscr{C})_{n}=\operatorname{Cat}_{\Delta}\left(\mathfrak{C}\left[\Delta^{n}\right], \mathscr{C}\right)$. 
That is, an $n$-simplex of $N(\mathscr{C})$ is a homotopy coherent diagram in $\mathscr{C}$ of shape $[n]$, that is, a map of graphs $[n] \rightarrow \mathscr{C}$ along with various homotopies between compositions, homotopies between these homotopies, and so forth. More specifically, $N(\mathscr{C})_{0}=\mathrm{Ob}(\mathscr{C}), N(\mathscr{C})_{1}$ is the set of arrows, and $N(\mathscr{C})_{2}$ is the set of all triples of morphisms $(f, g, h)$ together with a homotopy filling the triangle

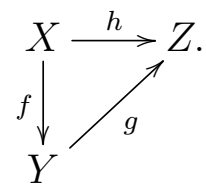

Example A.20 (3-simplices in the nerve). $N(\mathscr{C})_{3}$ is the set of all homotopy coherent diagrams of shape [3] in $\mathscr{C}$ :

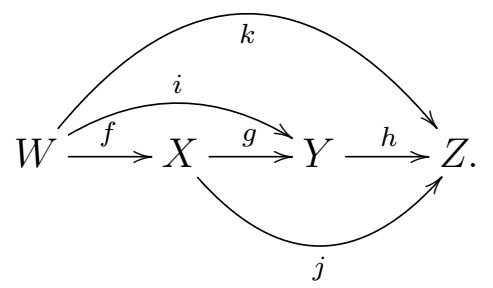

As usual, we have suppressed arrows like $g \circ f, h \circ g$, and $h \circ g \circ f$ in the figure, but these morphisms will play a role in the following. If this diagram commutes up to homotopy, then there exist homotopies

$$
\begin{aligned}
\alpha: g \circ f \simeq i, & \beta: h \circ g \simeq j, & \gamma: h \circ g \circ f \simeq k . \\
\delta: h \circ i \simeq k, & \varepsilon: j \circ f \simeq k . &
\end{aligned}
$$

This just means that the corresponding diagram in $\operatorname{Ho}(\mathscr{C})$ commutes. To say that the diagram is homotopy coherent means more, namely, that there exists a coherent system of homotopies: In addition to the homotopies $\alpha, \beta, \gamma, \delta, \varepsilon$ above there should exist homotopies $\left(\operatorname{rel} \partial \Delta^{1}\right)$

$$
\varepsilon *(\beta \circ f) \simeq \gamma \simeq \delta *(h \circ \alpha)
$$

These are all part of the data of a homotopy coherent diagram of shape [3].

\section{A.4.2 Further Elementary Notions}

The most important example of the simplicial nerve construction is the $\infty$ category $\mathcal{S}=N(\mathbf{K a n})$ of spaces, defined as the simplicial nerve of Kancomplexes with the simplicial enrichment given by $(109)$. 
Definition A.21. Morphisms $\phi, \psi \in \mathcal{C}_{1}$ are said to be homotopic or equivalent in case $\partial \phi=\partial \psi$ and there exists $\sigma \in \mathcal{C}_{2}$ with $\partial \sigma=\left(s_{0} b, \phi, \psi\right)$.

This amounts to saying that $[\phi]=[\psi]$ in he. A morphism $\phi$ is called an equivalence (and source and target are equivalent) if $[\phi]$ is an isomorphism when viewed as an arrow in he.

Definition A.22. A functor between $\infty$-categories $\mathcal{C}, \mathcal{D}$ is a simplicial map $f: \mathcal{C} \rightarrow \mathcal{D}$. It is said to be a categorical equivalence if:

1. Every $d \in \mathcal{D}_{0}$ is equivalent in $\mathcal{D}$ to some $f(c), c \in \mathfrak{C}_{0}$.

2. For any pair of objects $c_{1}, c_{2} \in \mathcal{C}_{0}, f$ induces a weak equivalence of simplicial sets

$$
\mathfrak{C}[f]: \operatorname{Map}_{\mathfrak{C}}\left(c_{1}, c_{2}\right) \rightarrow \operatorname{Map}_{\mathcal{D}}\left(f c_{1}, f c_{2}\right) .
$$

Definition A.23. Suppose $\mathcal{C}$ is an $\infty$-category. Then for any simplicial set $K$, the space $\mathbf{H o m}_{\mathbf{S e t}_{\Delta}}(K, \mathrm{C})$ is again an $\infty$-category and is denoted by $\operatorname{Fun}(K, \mathrm{C})$.

\section{A.4.3 The Join of $\infty$-Categories}

Let $K$ and $L$ be simplicial sets. Then the join $K \star L$ is obtained by putting $K$ and $L$ next to each other and joining all the simplices of $K$ with those of $L$ by lines (pointing in the direction of $L$ ), thus obtaining a simplex of one dimension higher. More precisely, setting $K_{-1}=\{*\}=L_{-1}$,

$$
(K \star L)_{n}=\coprod_{k=-1}^{n} K_{k} \times L_{n-k-1} .
$$

The face maps $d_{i}:(K \star L)_{n} \rightarrow(K \star L)_{n-1}$ are given by

$$
d_{i} \mid K_{k} \times L_{n-k-1}= \begin{cases}d_{i} \times \mathrm{id} & (i \leq k), \\ \mathrm{id} \times d_{i-k-1} & (i>k) .\end{cases}
$$

The degeneracy maps $s_{i}$ are given by the same formula - replacing $d$ by $s$ throughout. 


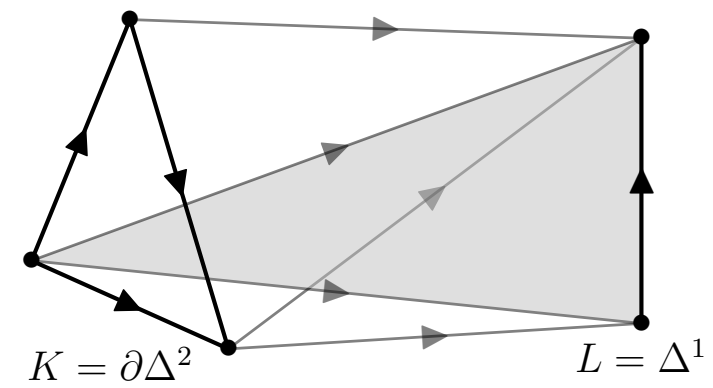

We have an isomorphism $\Delta^{i} \star \Delta^{j} \cong \Delta^{i+j+1}$. The join is obviously a functor. The non-degenerate $n$-simplices of $K \star L$ are given by all of the $\sigma \star \tau: \Delta^{n} \rightarrow K \star L$ for non-degenerate simplices $\Delta^{k} \stackrel{\sigma}{\rightarrow} K, \Delta^{n-k-1} \stackrel{\tau}{\rightarrow} L$.

It is not hard to check that the join of two $\infty$-categories is again an $\infty$ category. Moreover, for the classical join of ordinary categories (the disjoint union of the categories together with precisely one arrow from every object in $\mathcal{C}$ to every object in $\mathcal{D}$ ) we have

$$
N(\mathcal{C} \star \mathcal{D}) \cong N(\mathcal{C}) \star N(\mathcal{D}) .
$$

\section{A.4.4 Overcategories and Undercategories}

For ordinary categories, a functor $\mathcal{C} \star[0] \rightarrow \mathcal{D}$ is just a functor $F: \mathcal{C} \rightarrow \mathcal{D}$ with a co-cone on it and $\mathcal{D}_{F /}=\operatorname{Fun}_{F}(\mathcal{C} \star[0], \mathcal{D})$ is the category of co-cones from $F$, an arrow of which may be identified as an object of $\operatorname{Fun}_{F}(\mathcal{C} \star[1], \mathcal{D})$. If we take $\mathcal{C}=[0]$, then this is just the category $\mathcal{D}_{F(0) /}$ of objects under $F(0) \in \mathcal{D}$. We call $\mathcal{D}_{F /}$ the category of objects under the functor $F$, which may be identified with the comma category $\{F\} \downarrow \Delta$ for the diagonal functor $\Delta: \mathcal{D} \rightarrow \mathcal{D}^{\mathcal{C}}$. Of course, a colimit of $F$ is just an initial object in $\mathcal{D}_{F /}$.

Now suppose $f: K \rightarrow L$ is a simplicial map and $L$ an $\infty$-category. Then the $\infty$-category of objects of $L$ under $f$ is

$$
\left(L_{f /}\right)_{n}:=\left(\operatorname{Set}_{\Delta}\right)_{f}\left(K \star \Delta^{n}, L\right),
$$

where the subscript " $f$ " indicates that we are considering the subset of simplicial maps $K \star \Delta^{n} \rightarrow L$ that restrict to $f$ on $K$. This is indeed again an $\infty$-category. Moreover, $N\left(\mathcal{D}_{F /}\right) \cong N(\mathcal{D})_{N(F) /}$ so we have generalized the classical case. Dually, the $\infty$-category of objects of $L$ over $f$ is

$$
\left(L_{/ f}\right)_{n}=\left(\operatorname{Set}_{\Delta}\right)_{f}\left(\Delta^{n} \star K, L\right),
$$


which is to be thought of as a generalization of the category $\Delta \downarrow\{F\}$ of cones to $f$.

\section{A.4.5 Final and Initial Objects, Limits and Colimits}

Let $K$ be an $\infty$-category. An object $x \in K_{0}$ is said to be final/initial if it is final/initial when considered an object of the $\mathrm{h} S$-enriched category $\mathrm{h} K$. That is, $x$ is initial if for each object $y \in K_{0}$ the mapping space $\mathfrak{C}[K](x, y) \simeq *$ is contractible. Dually, $y \in K_{0}$ is final if for all $x \in K_{0}$ the space $\mathfrak{C}[K](x, y) \simeq *$.

For $\infty$-categories this is equivalent to the much more usable criterion of being strongly initial: Every $f: \partial \Delta^{n} \rightarrow K$ with $f(0)=x$ extends to $\Delta^{n}$. In other words, the canonical map $K_{x /} \rightarrow K$ is required to be a trivial fibration. Strongly final objects are defined similarly and are the same as final objects.

The full sub-category $K_{\text {initial }}=K \times_{N(h K)} N\left((h K)_{\text {initial }}\right)$ of initial objects in $K$ is contractible or empty. This is immediate since every $\partial \Delta^{n} \rightarrow K$ may be filled. More concretely,

$$
\left(K_{\text {initial }}\right)_{n}=\left\{f: \Delta^{n} \rightarrow K \mid f(0), \ldots, f(n) \text { initial }\right\} .
$$

Let $f: K \rightarrow L$ be a functor of $\infty$-categories. Then a colimit of $f$ is an initial object of $L_{f /}$ while a limit of $f$ is a final object of $L_{/ f}$. That is, a colimit of $f$ consists of a simplicial map

$$
\phi: K \star \Delta^{0} \rightarrow L
$$

that restricts to $f$ on $K$ having the following property: every map $K \star \partial \Delta^{n} \rightarrow L$ there exists the indicated extension

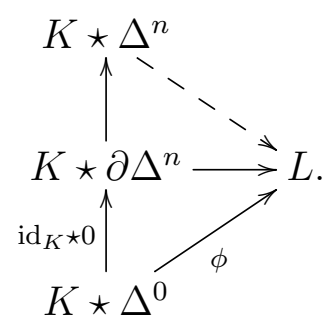

\section{A.4.6 coCartesian Fibrations}

Roughly speaking, a coCartesian fibration is a map $p: K \rightarrow L$ which may be thought of as a covariant functor $F$ on $L$ into the fibers of $p$ (which are $\infty$ categories) satisfying the axioms for a functor up to 'coherent homotopy' (in 
detail, $F$ is a simplicial functor $\left.\mathfrak{C}[L] \rightarrow \mathcal{C} a t_{\infty}\right)$. For each morphism $e: x \rightarrow y$ in $L$ one must present a sensible 'functor' $L_{x} \rightarrow L_{y}$. The functor $F$ is defined by 'fiber transport', that is, it associates to an initial point $a \in L_{x}$ the endpoint of a 'lift' of $e$. Of course, this endpoint should be unique up to contractible choice, so we need to choose special kinds of lifts that will guarantee this. The allowed choices for lifts are the $p$-coCartesian edges:

Suppose $p: K \rightarrow L$ is an inner fibration. An edge $e \in K_{1}$ is said to be $p$-coCartesian [Lur09, 2.4.1.4] in case for each $n \geq 2$ we may always find fillers as indicated:

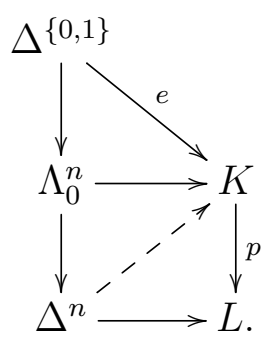

Example A.24 (Joyal / Theorem 1.2.4.3 in [Lur09]). Let $p: \mathcal{C} \rightarrow \Delta^{0}$ be an inner fibration. Then a $p$-coCartesian edge is precisely an equivalence in the $\infty$-category $\mathcal{C}$.

Definition A.25. An inner fibration $p: K \rightarrow L$ is said to be a coCartesian fibration if there is a sufficient supply of $p$-coCartesian edges: for every $f \in L_{1}$ and every $k \in K_{0}$ there exists a $p$-coCartesian edge $e \in K_{1}$ beginning at $k$.

Fiber transport along $p$-coCartesian edges of a coCartesian fibration $p$ : $\mathcal{C} \rightarrow \mathcal{D}$ determines a functor

$$
\mathrm{h} \mathcal{D} \rightarrow \operatorname{Ho}\left(\operatorname{Set}_{\Delta}\right)
$$

which on objects is given by taking $d \in \mathcal{D}$ to the fiber $p^{-1}(d)$.

Remark A.26. A characteristic of the $\infty$-category approach to higher categories is that coherence conditions are encoded by lifting properties and not made explicit (as, for example, for simplicial categories). Structure is presented as a (co)Cartesian fibration $p: \mathcal{C} \rightarrow K$ and the shape of $K$ determines the kind of structure we are dealing with. Under an abstract 
(un)straightening construction, $p$ corresponds to a simplicial functor indexed on a simplicial resolution $\mathfrak{C}[K]$ of $K$, and the data here is explicit.

\section{References}

[Ada72] J. F. Adams, Algebraic topology - a student's guide, Cambridge University Press, London, 1972, London Mathematical Society Lecture Note Series, No. 4. MR 0445484 (56 \#3824)

[Ada95]_ Stable homotopy and generalised homology, Chicago Lectures in Mathematics, University of Chicago Press, Chicago, IL, 1995, Reprint of the 1974 original. MR 1324104 (96a:55002)

[AM10] Marcelo Aguiar and Swapneel Mahajan, Monoidal functors, species and Hopf algebras, CRM Monograph Series, vol. 29, American Mathematical Society, Providence, RI, 2010, With forewords by Kenneth Brown and Stephen Chase and André Joyal. MR 2724388 (2012g:18009)

[APS75] M. F. Atiyah, V. K. Patodi, and I. M. Singer, Spectral asymmetry and Riemannian geometry. I, Math. Proc. Cambridge Philos. Soc. 77 (1975), 43-69. MR 0397797 (53 \#1655a)

[Bar02] Michael Barr, Acyclic models, CRM Monograph Series, vol. 17, American Mathematical Society, Providence, RI, 2002. MR 1909353 (2003k:18017)

[BG13] Ulrich Bunke and David Gepner, Differential function spectra, the differential Becker-Gottlieb transfer, and applications to differential algebraic K-theory, ArXiv e-prints 1306.0247 (2013).

[BKS10] Ulrich Bunke, Matthias Kreck, and Thomas Schick, A geometric description of differential cohomology, Ann. Math. Blaise Pascal 17 (2010), no. 1, 1-16. MR 2674652 (2011j:55007)

[Bro62] Edgar H. Brown, Jr., Cohomology theories, Ann. of Math. (2) 75 (1962), 467-484. MR 0138104 (25 \#1551) 
[Bry08] Jean-Luc Brylinski, Loop spaces, characteristic classes and geometric quantization, Modern Birkhäuser Classics, Birkhäuser Boston Inc., Boston, MA, 2008, Reprint of the 1993 edition. MR 2362847 (2008h:53155)

[BS09] Ulrich Bunke and Thomas Schick, Smooth K-theory, Astérisque (2009), no. 328, 45-135 (2010). MR 2664467 (2012a:19015)

[BS10]_, Uniqueness of smooth extensions of generalized cohomology theories, J. Topol. 3 (2010), no. 1, 110-156. MR 2608479 (2011e:55011)

[BSSW09] Ulrich Bunke, Thomas Schick, Ingo Schröder, and Moritz Wiethaup, Landweber exact formal group laws and smooth cohomology theories, Algebr. Geom. Topol. 9 (2009), no. 3, 1751-1790. MR 2550094 (2011d:55005)

[BT82] Raoul Bott and Loring W. Tu, Differential forms in algebraic topology, Graduate Texts in Mathematics, vol. 82, SpringerVerlag, New York, 1982. MR 658304 (83i:57016)

[BT06] Mark Brightwell and Paul Turner, Relative differential characters, Comm. Anal. Geom. 14 (2006), no. 2, 269-282. MR 2255012 (2007e:53022)

[Bun12] Ulrich Bunke, Differential cohomology, ArXiv e-prints 1208.3961 (2012).

[CS85] Jeff Cheeger and James Simons, Differential characters and geometric invariants, Geometry and topology (College Park, Md., 1983/84), Lecture Notes in Math., vol. 1167, Springer, Berlin, 1985, pp. 50-80. MR 827262 (87g:53059)

[DK01] James F. Davis and Paul Kirk, Lecture notes in algebraic topology, Graduate Studies in Mathematics, vol. 35, American Mathematical Society, Providence, RI, 2001. MR 1841974 (2002f:55001)

[Dol62] Albrecht Dold, Relations between ordinary and extraordinary cohomology, Colloq. algebr. Topology, Aarhus (available in [Ada72]) (1962), 2-9. 
[Dol95]—, Lectures on algebraic topology, Classics in Mathematics, Springer-Verlag, Berlin, 1995, Reprint of the 1972 edition. MR 1335915 (96c:55001)

[DS11] Daniel Dugger and David I. Spivak, Rigidification of quasicategories, Algebr. Geom. Topol. 11 (2011), no. 1, 225-261. MR 2764042 (2012f:55025)

[FL10] Daniel S. Freed and John Lott, An index theorem in differential K-theory, Geom. Topol. 14 (2010), no. 2, 903-966. MR 2602854 (2011h:58036)

[FQ93] Daniel S. Freed and Frank Quinn, Chern-Simons theory with finite gauge group, Comm. Math. Phys. 156 (1993), no. 3, 435-472. MR 1240583 (94k:58023)

[Fre95] Daniel S. Freed, Classical Chern-Simons theory. I, Adv. Math. 113 (1995), no. 2, 237-303. MR 1337109 (96h:58019)

[Fre02] Classical Chern-Simons theory. II, Houston J. Math. 28 (2002), no. 2, 293-310, Special issue for S. S. Chern. MR 1898192 (2003f:55022)

[Fre09] , Remarks on Chern-Simons theory, Bull. Amer. Math. Soc. (N.S.) 46 (2009), no. 2, 221-254. MR 2476413 (2010a:58031)

[Gaj97] Paweł Gajer, Geometry of Deligne cohomology, Invent. Math. 127 (1997), no. 1, 155-207. MR 1423029 (98f:14012)

[GJ09] Paul G. Goerss and John F. Jardine, Simplicial homotopy theory, Modern Birkhäuser Classics, Birkhäuser Verlag, Basel, 2009, Reprint of the 1999 edition [MR1711612]. MR 2840650

[Hir03] Philip S. Hirschhorn, Model categories and their localizations, Mathematical Surveys and Monographs, vol. 99, American Mathematical Society, Providence, RI, 2003. MR 1944041 (2003j:18018)

[Hov99] Mark Hovey, Model categories, Mathematical Surveys and Monographs, vol. 63, American Mathematical Society, Providence, RI, 1999. MR 1650134 (99h:55031) 
[HS05] M. J. Hopkins and I. M. Singer, Quadratic functions in geometry, topology, and M-theory, J. Differential Geom. 70 (2005), no. 3, 329-452. MR 2192936 (2007b:53052)

[HSS00] Mark Hovey, Brooke Shipley, and Jeff Smith, Symmetric spectra, J. Amer. Math. Soc. 13 (2000), no. 1, 149-208. MR 1695653 (2000h:55016)

[JS93] André Joyal and Ross Street, Braided tensor categories, Adv. Math. 102 (1993), no. 1, 20-78. MR 1250465 (94m:18008)

[Kel74] G. M. Kelly, Doctrinal adjunction, Category Seminar (Proc. Sem., Sydney, 1972/1973), Springer, Berlin, 1974, pp. 257-280. Lecture Notes in Math., Vol. 420. MR 0360749 (50 \#13196)

[Lee03] John M. Lee, Introduction to smooth manifolds, Graduate Texts in Mathematics, vol. 218, Springer-Verlag, New York, 2003. MR 1930091 (2003k:58001)

[Lei04] Tom Leinster, Higher operads, higher categories, London Mathematical Society Lecture Note Series, vol. 298, Cambridge University Press, Cambridge, 2004. MR 2094071 (2005h:18030)

[Lüc05] Wolfgang Lück, Algebraische Topologie: Homologie und Mannigfaltigkeiten, 1. Aufl. ed., Vieweg, Wiesbaden, 2005.

[Lur07a] Jacob Lurie, Derived Algebraic Geometry II: Noncommutative Algebra, arXiv preprint math/0702299 (2007).

[Lur07b] - Derived Algebraic Geometry III: Commutative algebra, arXiv preprint math/0703204 (2007).

[Lur07c] — Stable Infinity Categories, arXiv preprint math.CT/0608228 (2007).

[Lur09] - Higher topos theory, Annals of Mathematics Studies, vol. 170, Princeton University Press, Princeton, NJ, 2009. MR 2522659 (2010j:18001)

[Lur11]_ Higher algebra, Prepublication book draft. Available from http://www. math.harvard.edu/ lurie (2011). 
[Mal11] Cary Malkiewich, The stable homotopy category, Available from http://math.stanford.edu/ ${ }^{\sim}$ carym (2011).

[May92] J. Peter May, Simplicial objects in algebraic topology, Chicago Lectures in Mathematics, University of Chicago Press, Chicago, IL, 1992, Reprint of the 1967 original. MR 1206474 (93m:55025)

[McC01] John McCleary, A user's guide to spectral sequences, second ed., Cambridge Studies in Advanced Mathematics, vol. 58, Cambridge University Press, Cambridge, 2001. MR 1793722 (2002c:55027)

[ML98] Saunders Mac Lane, Categories for the working mathematician, second ed., Graduate Texts in Mathematics, vol. 5, SpringerVerlag, New York, 1998. MR 1712872 (2001j:18001)

[Qui67] Daniel G. Quillen, Homotopical algebra, Lecture Notes in Mathematics, No. 43, Springer-Verlag, Berlin, 1967. MR 0223432 (36 \#6480)

[Sch07] Stefan Schwede, An untitled book project about symmetric spectra, Preprint available from http://www.math.uni-bonn.de/ schwede 7 (2007), no. 9.

[SS00] Stefan Schwede and Brooke Shipley, Algebras and modules in monoidal model categories, Proc. London Math. Soc. (3) 80 (2000), no. 2, 491-511. MR 1734325 (2001c:18006)

[SS03a] _ Equivalences of monoidal model categories, Algebr. Geom. Topol. 3 (2003), 287-334 (electronic). MR 1997322 (2004i:55026)

[SS03b] Stable model categories are categories of modules, Topology 42 (2003), no. 1, 103-153. MR 1928647 (2003g:55034)

[SS08] James Simons and Dennis Sullivan, Axiomatic characterization of ordinary differential cohomology, J. Topol. 1 (2008), no. 1, 45-56. MR 2365651 (2009e:58035)

[Swi75] Robert M. Switzer, Algebraic topology-homotopy and homology, Springer-Verlag, New York, 1975, Die Grundlehren der mathematischen Wissenschaften, Band 212. MR 0385836 (52 \#6695) 
[tD08] Tammo tom Dieck, Algebraic topology, EMS Textbooks in Mathematics, European Mathematical Society (EMS), Zürich, 2008. MR 2456045 (2009f:55001)

[Upm11] Markus Upmeier, Products in generalized differential cohomology.

[Upm12]_, A bicategory approach to differential cohomology.

[Whi62] George W. Whitehead, Generalized homology theories, Trans. Amer. Math. Soc. 102 (1962), 227-283. MR 0137117 (25 \#573)

Mathematisches Institut, Georg-August Universität Göttingen, Bunsenstrasse 3-5, D-37073 Göttingen

E-mail address: upmeier@ uni-math.gwdg.de 


\section{Curriculum Vitae}

Dipl. Math. Markus A. Upmeier

PERSONAL DATA

Born November 19, 1985 in Lawrence, KS (USA)

Citizenship Germany, United States of America

Address Von-Ossietzky-Str. 16

D-37085 Göttingen

Phone $\quad(+49) 551 / 2934147$

Homepage www.uni-math.gwdg.de/upmeier

Email upmeier@uni-math.gwdg.de

EDUCATION

PhD Student at the University of Göttingen

since $11 / 2010$

Research Training Group 1493 'Mathematical Structures in Modern Quantum

Physics', under supervision of Prof. Dr. T. Schick.

Diploma in Mathematics and Physics

$09 / 2010$

University of Göttingen, total score 1.0, graduated with honors. Thesis title:

'A Generalized Miraculous Cancellation Formula for Indices of Dirac Opera-

tors'. Thesis grade: 1.0

Gymnasium Philippinum (grammar school)

$07 / 2005$

Marburg an der Lahn, A-levels, mark 1.3

Honors, SchOlarships, AND Awards

Stipend of the DFG (German Science Foundation)

since 2011

RTG1493 'Mathematical Structures in Modern Quantum Physics'

Diploma Honors Award 'Graduated with Excellence'

$10 / 2010$

Awarded by the Mathematical Institute, Göttingen

Stipend of the 'Studienstiftung des Deutschen Volkes'

$2008-2010$

A prestigious scholarship for university students of all disciplines

Awarded 2 ${ }^{\text {nd }}$ Place at the 'Bundeswettbewerb Mathematik'

$09 / 2005$

Germany-wide high school math competition

High School Honors Awards

$06 / 2005$

Of the German Physics Foundation (DPG) as well as the math honors award

'Hans-Heinrich Behr Preis' 